\title{
FOLK DEFINITIONS AS A MODEL FOR SIGN LANGUAGE DICTIONARY DEFINITIONS: \\ A user-focused case study of the Online Dictionary of New Zealand Sign Language
}

\author{
by
}

Mireille Vale

A thesis submitted to Victoria University of Wellington in fulfillment of the requirements for the degree of Doctor of Philosophy 



\begin{abstract}
This thesis addresses the question whether signed definitions, made possible by advances in electronic lexicography, should be introduced to sign language dictionaries. The thesis comprises four interrelated studies investigating different aspects of this question through a user-focused case study of the Online Dictionary of New Zealand Sign Language (ODNZSL).
\end{abstract}

A preliminary study investigated current use of the ODNZSL in order to identify what user needs signed definitions might fulfil. The study drew on two data sets: website log data for the ODNZSL, and a think-aloud protocol and interview with representatives of user groups. Results showed that in addition to a large volume of casual browsers, the most frequent and intensive users of the dictionary are beginner and intermediate students of New Zealand Sign Language (NZSL). These (hearing) language learners mostly search for frequent vocabulary with the aims of language production and vocabulary learning. Findings also identified reasons for unsuccessful dictionary consultations that may impact on the effectiveness of definitions.

In the second study, a review of ODNZSL entries highlighted categories of lexical items for which the current description through English glosses, examples, and usage notes is inadequate. A test was developed to assess whether these categories of signs were problematic for the user group identified in the first study: hearing intermediate learners of NZSL. Twenty-one participants took a computer-based error correction test with both comprehension and production sections comprising fifty items in six different categories: culture-bound; idiomatic; polysemous; metaphoric/metonymic; vocabulary type / word class; and other. Quantitative results indicated that a small number of test items were problematic, but that none of the test categories were good predictors of the difficulties learners experienced. A qualitative examination identified linguistic factors and issues with the current dictionary information that may be improved by the addition of signed definitions.

The central proposition tested in the third study was that folk definitions-informal explanations of sign meaning by Deaf sign language users-can be applied as a template for dictionary definitions. This study took fifteen of the signs that were identified as problematic for learners in the previous study, and asked thirteen Deaf NZSL users to explain the meaning of these signs. A qualitative analysis found that the folk definitions by different NZSL users shared common semantic categories and embedded information about situational and 
sociolinguistic variation as well as grammatical structures. Some semantic relationships that occur frequently in spoken language folk definitions, such as exemplification and synonymy, were also common in signed folk definitions. Other semantic relationships such as attribution, function, operation, and spatial relationships occurred less frequently because they were inherent in the sign construction. Due to the bilingual status of the participants, many folk definitions included reference to English words in the form of mouth patterns and fingerspelling.

In the fourth study, twelve pilot dictionary definitions were created on the basis of common features found in the folk definitions and an evaluation of definition formats by Deaf NZSL users. The error correction test from the second study was repeated with a new cohort of intermediate NZSL learners. This time twelve test items were accompanied by a pilot definition; for the remaining items participants were shown a video example sentence from the ODNZSL entry. Results showed no significant improvements in scores for the test items with definitions. However, feedback from test participants showed that the definitions were comprehensible and perceived as valuable for language learning.

The overall conclusion of these studies is that a selective approach should be taken to introducing signed definitions in existing multifunctional sign language dictionaries. For hearing learners of sign language, signed definitions do not meet immediate communicative (comprehension and production) needs, but they may contribute to wider vocabulary learning goals.

The main contribution of this thesis is that it suggests a user-focused methodology for creating signed definitions, driven by evidence from the first empirical user study of an online sign language dictionary and therefore taking into account the particular challenges of sign language lexicography. Furthermore, the analysis of features of signed folk definitions contributes to the semantic description of sign languages. 


\section{Table of Contents}

Abstract $\ldots$

Table of Contents

Acknowledgments. . .

Copyright permissions and publication relating to this thesis . . . . . . . . . . . . . . . . . viii

List of Figures . . . . . . . . . .

List of Tables . . . . . . . .

List of Abbreviations.

Note on the representation of signs . .

Note on terminology $\ldots \ldots \ldots \ldots$

Chapter 1 - Introduction $\ldots \ldots$

Introduction

1.1 - Motivation for the thesis . . . . . .

1.2 - Aims and significance of the thesis .

1.3 - Overview of the thesis . .

Chapter 2 - Sign languages and the sign language lexicon _. _ _ _ _ . 9

Introduction . . .

2.1 - The sociolinguistic context _ _ _ _ _ 9

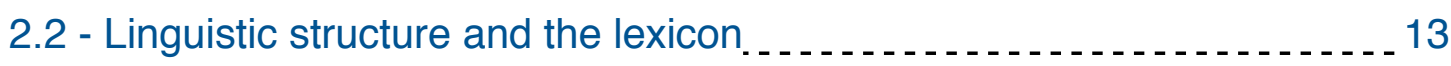

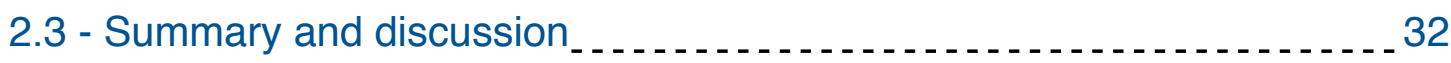

2.4 - Conclusion 35

Chapter 3 - Sign language lexicography . . . . . 37

Introduction . .

3.1 - History and purposes of sign language dictionaries . . _ . _ _ _ 37

3.2 - The format of sign language dictionaries . . . . . . . 40

3.3 - Sign language dictionary types . . . . . . . . . . . . . . . . . . . 44

3.4 - Dictionary typology: some distinctions . . . . . . . . 45

3.5 - Conclusion 60

Chapter 4 - A user study of an online sign language dictionary _ _ _ 61

Introduction . . . . 61

4.1 - User studies in lexicography _ . _ _ _ _ _ _ _ _ _ _ _ _ _ _ 62

4.2 - User studies of sign language dictionaries . . _ _ _ _ _ _ _ _ _ 66 
4.3 - Research questions . _ _ _ _ _ 69

4.4 - Methodology of user research . _ _ _ 70

4.5 - Method . . . . . 72

4.6 - Results and discussion $\ldots$

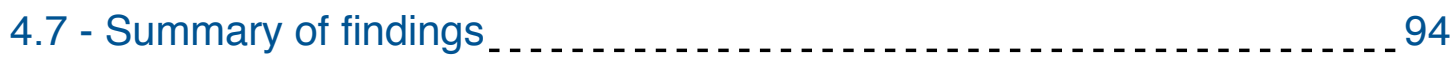

4.8 - Limitations.

4.9 - Conclusion $\ldots 96$

Chapter 5 - Evaluating the user need for definitions _ _ _ 101

Introduction

5.1 - Dictionaries and language learning . _ _ _ 102

5.2 - Equivalents or glosses _ _ _ _ _ _ _ _ 108

5.3 - Examples. . . .

5.4 - Research question

5.5 - Methodology of dictionary tests

5.6 - Method. . . . 130

5.7 - Results and discussion $\ldots . \ldots \ldots$

5.8 - Summary of findings $\ldots$

5.9 - Limitations . _ _ _ n 152

5.10 - Conclusion

Chapter 6 - Folk definitions

Introduction

6.1 - Folk definitions as data

6.2 - Spoken definitions and explanations . . . . . . . 158

6.3 - Folk definitions in dictionaries . . . . . _ _ 161

6.4 - Signed definitions and explanations .

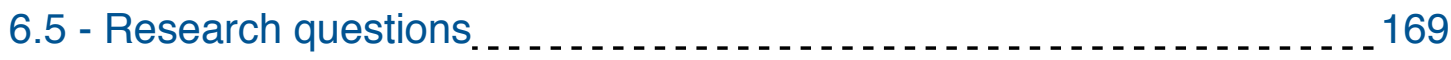

6.6 - Methodology of folk definition studies . _ _ _ 169

6.7 - Method. . . . . 170

6.8 - Results and discussion

6.9 - Summary of findings . . . . 210

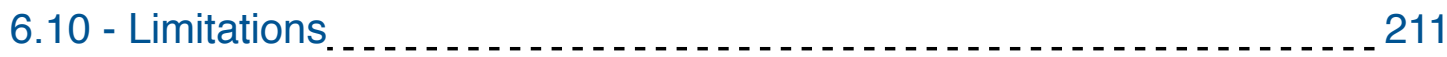

6.11 - Conclusion

Chapter 7 - From folk definition to dictionary definition . _ _ _ 217

Introduction 
7.1 - Deaf perspectives on definition formats .

7.2 - Developing dictionary definitions: editing and filming _ . _ . _ . _ _ _ _ 227

7.3 - Testing the effectiveness of dictionary definitions. . . . . _ _ _ 236

7.4 - Conclusion . . . 246

Chapter 8 - Conclusion

Introduction

8.1 - Summary of research findings

8.2 - Should signed definitions be added to sign language dictionaries? _. 252

8.3 - Original contributions . . . . 252

8.4 - Limitations and future research . _ _ _ _ _ _ _ _ _ _ _ _ _ _ 254

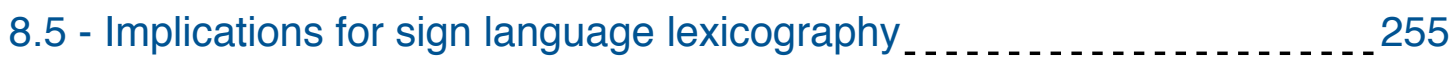

8.6 - Conclusion . .

References

Dictionaries and glossaries $\ldots \ldots \ldots$

Other references

\section{Appendices (on CD)}

\section{Appendix A: Background material to Chapter 4}

A1 Information sheet: Think Aloud Protocol

A2 Consent form: Think Aloud Protocol

A3 Schedule: Think Aloud Protocol

\section{Appendix B: Background material to Chapter 5}

B1 Information sheet: Online error correction test

B2 Test instrument

\section{Appendix C: Background material to Chapter 6}

C1 Information sheet: Eliciting folk definitions

C2 Consent form: Eliciting folk definitions

C3 Video clips for Figure 6.2: NZSL folk definitions using synonymy

C4 Video clips for Table 6.6: Examples of inflected signs in NZSL folk definitions

\section{Appendix D: Background material to Chapter 7}

D1 Definition evaluation rating sheet: Focus group

D2 Facilitator's notes: Focus group

D3 Pilot dictionary definition videos

D4 Modified test instrument

Appendix E: Ethics approval 


\section{Acknowledgments}

My very first thanks go to the Deaf community in New Zealand and worldwide for allowing me to be a small part of a signing world. Particular thanks are due to the Deaf people and hearing NZSL learners who generously gave their time to be participants in my research. I especially want to acknowledge David Brien and the late Mary Brennan at Durham University, and David McKee and Rachel McKee at Victoria University of Wellington, for their co-directorship of the Deaf Studies Research Units at these respective universities, where it has been my privilege to experience the development of two sign language dictionaries and to work with colleagues whose insightfulness and tireless hard work have been a constant inspiration.

To my supervisors, Rachel McKee and Frank Boers, thank you for your expertise and your patience, good humour and encouragement throughout the process. Also thank you for letting me go ahead with a rather ambitious plan of work and not saying 'I told you so' whenever it proved to be too much.

I am grateful to Victoria University of Wellington for the Doctoral Scholarship and Submission Scholarship that enabled me to complete this thesis. Financial support provided by the Faculty of Humanities and Social Sciences in the form of research and conference attendance grants is also gratefully acknowledged.

Thank you to:

- $\quad$ Daniel Harborne, Lynette Pivac and Sara Pivac Alexander for acting as facilitators during interviews and focus groups;

- James Berry, Mark Berry, Sara Pivac Alexander and Sonia Pivac for being the NZSL models for research materials and figures in this thesis;

- $\quad$ Delys Magill, George Major and Susie Ovens for help with administering the test at AUT University.

Lizzie Towl of the Faculty of Graduate Research and all the Shut Up and Write / Thesis Bootcamp crew are responsible for $90 \%$ of the productive writing time on this thesis. Thanks especially to Keely, Louise, Evan, Abigael, Ha and Nancy for companionship, baking, 2-for-1 eggs and ritual sobs. To all my fellow LALS PhD students-Thesis Group, Vocab Group, social nights and quiz nights have been a huge part of making me feel like a 'real' student. 
And finally, a heartfelt thank you to my parents Anneke and Jan, and to my brothers, sister and extended family in the Netherlands for support from afar; to my parents-in-law Brenda and Robert for practical and moral support; to my daughter Laura for her constant encouragement and her advice to just keep writing 'words, words, words'; and last but not least to my husband Will, for being a steadfast beacon in the chaos, a shoulder to cry on, and for keeping things running at home while putting up with my procrastinating habits with surprisingly good grace. 


\section{Copyright permissions}

- Permission to reproduce material from the Dictionary of British Sign Language / English (Brien, 1992) in figure 3.1 was granted by Faber \& Faber Ltd. under license P160505/028.

- Permission to reproduce material from the British Sign Language Glossaries of Curriculum Terms (http://www.ssc.education.ed.ac.uk/BSL/list.html) in figure 7.1 was granted by Dr. John Ravenscroft, Head of the Scottish Sensory Centre. (C) University of Edinburgh.

- Permission to reproduce material from the Signs 2 Go online BSL course (http:// www.signs2go.eu/) in figure 7.2 was granted by Lynne Barnes, Department of Education and Social Science, University of Central Lancashire.

- Material from the Online Dictionary of New Zealand Sign Language (http:// nzsl.vuw.ac.nz) (McKee et al., 2011) reproduced in this thesis is licensed under a Creative Commons Attribution-NonCommercial-ShareAlike 3.o Unported License.

\section{Publication relating to this thesis}

The user study reported on in Chapter 4 also forms the basis of the following published paper:

Vale, M. (2015). A study of the users of an online sign language dictionary. In I. Kosem, M. Jakubiček, J. Kallas, \& S. Krek (Eds.), Electronic lexicography in the 21st century: linking lexical data in the digital age. Proceedings of the eLex 2015 conference, 11-13 August 2015, Herstmonceux Castle, United Kingdom. (pp. 281-303). Ljubljana / Brighton: Trojina, Institute for Applied Slovene Studies / Lexical Computing Ltd. Retrieved from https://elex.link/elex2015/ proceedings/eLex_2015_18_Vale.pdf 


\section{List of Figures}

Figure 2.1: Iconicity-selection, schematisation and encoding (following Taub, 2001)

Figure 2.2: Iconic signs derived from schematisation of different features

Figure 2.3: NZSL signs using the fingers to represent a person's legs

Figure 2.4: Double mapping in the NZSL sign REMEMBER

Figure 2.5: A lexicalised polycomponential sign-BUNGY-JUMP

Figure 2.6: A loan translation compound in NZSL-WORKSHOP

Figure 2.7: Three forms of a polycomponential NZSL sign-GIVE

Figure 2.8: An NZSL sign showing number incorporation-ONE-YEAR-OLD 34

Figure 3.1: An entry in the Dictionary of British Sign Language / English

Figure 4.1: The ODNZSL home page

Figure 4.2: Search methods in the ODNZSL

Figure 4.3: Search results display in the ODNZSL

Figure 4.4: Individual dictionary entry in the ODNZSL

Figure 4.5: Multiple search results for 'soccer'

Figure 5.1: Main and secondary glosses for the ODNZSL entry BUILD

Figure 5.2: Signs referring to people in terms of their hearing status and relationship to the Deaf community

Figure 5.3: A partly-lexicalised sign in which the handshape denotes small round objects, especially food

Figure 5.4: Collocations with the sign DEAF as multi-sign headwords in the ODNZSL

Figure 5.5: Three signs with different collocations-GROW

Figure 5.6: ODNZSL entries with English equivalents drawn from a different register

Figure 5.7: ODNZSL entry with English equivalents belonging to a different word class

Figure 5.8: Idiomatic expressions with a similar semantic content in NZSL and English

Figure 5.9: Idiomatic expressions with a different semantic content in NZSL and English

Figure 5.10: An example providing a definition of the sign DIRECTIONAL-VERB

Figure 5.11: Examples distinguishing between two senses of the sign phrase (CULTURALLY / TOTALLY) DEAF

Figure 5.12: A created example providing a context for the sign HUMILIATED 
Figure 5.14: Examples illustrating Deaf cultural frames for the signs HEARING 1 and HEARING 2

Figure 5.15: An example for the sign PLAIN containing potentially distracting implicit cultural information

Figure 5.16: Format of a question in the comprehension block of the online test 140

Figure 5.17: Format of a question in the production block of the online test 141

Figure 5.18: A polycomponential, partly specified sign in NZSL 149

Figure 5.19: Test item AC4 KEEP-INSIDE-ONESELF 150

Figure 6.1: Semantic categories used in NZSL folk definitions for 15 target signs 177

Figure 6.2: NZSL folk definitions using synonymy 178

Figure 6.3: NZSL folk definitions using exemplification $\quad 184$

Figure 6.4: Comparison of related signs through exemplification 186

Figure 6.5: Selectional restrictions for two signs: DECREASE 1 and DECREASE $2 \quad 187$

Figure 6.6: Comparison between the sign phrases FULLY DEAF and STRONGLY / VERY DEAF

Figure 6.7: NZSL folk definitions using antonymy

Figure 6.8: Signs used in the NZSL folk definitions to indicate register or situations of use

194

Figure 6.9: Comments about register in NZSL folk definitions

Figure 6.10: Two variant forms of the sign PUT-ASIDE in formal and informal production

Figure 6.11: Comments related to variation in NZSL folk definitions

Figure 6.12: Two signs ASK in NZSL $\quad 200$

Figure 6.13: Simultaneous production of the target sign and referential pointing 205

Figure 6.14: List buoys in NZSL 206

Figure 6.15: Grammatical inflection labels in the ODNZSL 215

Figure 7.1: Definition of ASTEROID in the BSL Glossaries of Curriculum Terms 221

Figure 7.2: Contextualised presentation of signed explanation in Signs2Go 221

Figure 7.3: Correspondences between rating criteria for the top rated folk definitions

223

Figure 7.4: References to 'a person' in the pilot dictionary definitions 234

Figure 7.5: Revised test item format (production section) including an example or definition video

Figure 7.6: Revised test instructions for comprehension and production parts 


\section{List of Tables}

Table 4.1: Participants for the interview / Think Aloud Protocol

Table 4.2: Participants' use and awareness of sign language dictionaries $\quad 80$

Table 4.3: New vs. returning users to the ODNZSL 82

Table 4.4: Top 25 search terms in the ODNZSL 86

Table 4.5: Views of the different elements for the ODNZSL entry PLAY 89

Table 5.1: Categories of test items 131

Table 5.2: Test items in category 1-Culture-bound 132

Table 5.3: Test items in category 2-Idiomatic 133

Table 5.4: Test items in category 3-Polysemous 134

Table 5.5: Test items in category 4-Metaphoric / metonymic 135

Table 5.6: Test items in category 5-Vocabulary type / word class 136

$\begin{array}{ll}\text { Table 5.7: Test items in category 6-Other } & 137\end{array}$

Table 5.8: Number of test items per mean score of correct responses 143

Table 5.9: Analysis of Variance of test item categories $\quad 144$

Table 5.10: Test items with a mean score of $50 \%$ or less 145

Table 5.11: Test items with a lower mean score for correction than for error identification 149

Table 6.1: 15 stimulus items used to elicit folk definitions 171

Table 6.2: Demographics of participants in the folk definition elicitation study 172

Table 6.3: Semantic relationship types of folk definitions (Adapted from Casagrande and Hale, 1967)

Table 6.4: Attributive folk definitions compared with attributive features inherent in NZSL signs

Table 6.5: A comparison of the register of signs related to 'talk' through the contexts referred to in NZSL folk definitions

Table 6.6: Examples of inflected signs in NZSL folk definitions 201

Table 6.7: Signs used to signal folk definitions in the NZSL data 205

Table 7.1: Twelve pilot dictionary definition videos 231

Table 7.2: Two-way factorial Analysis of Variance of modified and unmodified test items in Test 1 and Test 2

Table 7.3: Comparison of Test 1 and Test 2 error correction scores for modified test items 


\section{List of Abbreviations}

\section{Sign languages}

ASL American Sign Language

Auslan Australian Sign Language

BSL British Sign Language

DGS German Sign Language

LIS Italian Sign Language

NZSL New Zealand Sign Language

SLN Sign Language of the Netherlands

Dictionaries (see section Dictionaries and glossaries for complete references)

COBUILD Collins COBUILD English Language Dictionary

DASL Dictionary of American Sign Language on Linguistic Principles

DBSL/E Dictionary of British Sign Language / English

ODNZSL Online Dictionary of New Zealand Sign Language

Other abbreviations

TAP Think Aloud Protocol

L1 First language

L2 Second language 


\section{Note on the representation of signs}

In this thesis, signs will be represented in several ways. Where it was necessary to represent signs in static form, a combination of still images or drawings and written glosses has been used. There are a number of disadvantages to representing signs through glosses, especially when the focus is on discussing the semantic and grammatical function of signs (see also section 3.2.1 - Representing signs without a written form). Wherever possible, I have therefore provided links to video material. Wherever a dictionary entry exists in the Online Dictionary of New Zealand Sign Language (http://nzsl.vuw.ac.nz; McKee et al., 2011) for a particular sign, the gloss(es) used in this thesis will correspond with the main English equivalent(s) in the dictionary entry. Each dictionary entry contains a video demonstrating the production of the sign in 'citation form'.

In order to preserve the anonymity of my research participants, I have regretfully been unable to show their original signed folk definitions. Short illustrative examples of signs discussed in Chapter 6 - Folk definitions were re-filmed with a sign model. Longer extracts illustrating aspects such as register, variation and cultural identity have been shown in English translation only. The original video samples will be available to examiners on request. Translations will be shown in italics.

Other transcription conventions I have followed are listed below.

- Signs are shown by glosses in small capitals, e.g. DEAF.

- Multiple words describing a single sign are hyphened, e.g. KEEP-IN-MIND.

- When a dictionary entry for a sign has more than one main English equivalent, all main equivalents are shown in the gloss, e.g. ASK / REMIND / TELL.

- Fingerspelled words are shown by separating each letter in the gloss with a hyphen, e.g. b-e-a-n.

- Mouthings are shown in single quotation marks and italicised, e.g. 'develop'.

- Polycomponential ('classifier') signs are preceded by CL:, e.g. CL:PERSON 


\section{Note on terminology}

It has been common practice in Deaf Studies and related fields to follow Woodward's (1975) use of the word 'deaf' (with a small initial letter 'd') to refer to the audiological aspects of deafness, i.e. the inability to hear sound, in contrast with the word 'Deaf' (with a capital) referring to the sociocultural aspects of Deafness, i.e. when the focus is on a person's social identity as a Deaf person, or on associated institutions or practices. In this thesis I have followed this practice in order to highlight similarities between Deaf people and other communities who are united through a shared language and culture. However, I am conscious that this useful distinction may obscure much more complex realities of language use and multiple identities and communities. These realities are reflected in the folk definitions of certain signs related to d/Deafness that will be reported on in this thesis. In using this written convention I therefore do not mean to imply that the distinction is binary and I acknowledge Woodward and Horejes' (2016) observation that individuals can be, and often are, both 'deaf' and 'Deaf' at the same time. 


\section{Chapter 1: Introduction}

\section{Introduction}

Dictionaries come in many shapes, from multi-volume tomes to pocket-sized travel companions, and increasingly also in electronic form. The content of dictionaries is equally varied: they may contain words in one, two, or several languages, definitions, examples of use, grammatical labels, pronunciation guides, etymologies, notes, and illustrations; and although this information is traditionally presented in alphabetical order, it may also be organised differently. In undertaking the complex task of making a dictionary, lexicographers are increasingly guided in their decisions about format and content by focusing first and foremost on the dictionary user (e.g. Hartmann, 1989; Atkins \& Rundell, 2008; Tarp, 2009; Nesi, 2013 b).

In documenting the lexicon of a language, dictionaries also have broader linguistic, cultural and political functions. With regard to sign languages, a report by the World Federation of the Deaf on Deaf people and human rights (Haualand \& Allen, 2009) noted that in addition to facilitating communication between sign language users and the surrounding spoken language community, a sign language dictionary could be "a fundamental tool to both promote the status of and enhance the use of sign language......] and also serve as documentation of the existence of a sign language" (p. 23). The increased recognition of the linguistic status of sign languages since the 196os, arising from the pioneering work of Stokoe (1960), and the corresponding wider visibility of sign language users in society have seen a parallel rise in the number of sign language dictionaries. However, the diversification in formats in response to the needs of different users that was observed in dictionaries in general has not happened in the case of sign language dictionaries.

Particularly noteworthy is the fact that no monolingual sign language dictionaries have appeared, and that the use of sign languages as dictionary metalanguages has been severely restricted. Although the technological capabilities of online multimedia sign language dictionaries have led to the inclusion of longer stretches of signed information on video, including signed example sentences, to date a key element of sign description - the dictionary definition-has remained largely absent from generic descriptive sign language dictionaries, for reasons that will be expanded on in this thesis. 
This thesis will present a case study of a modern online sign language dictionary to examine the potential role of signed dictionary definitions and to consider the form such definitions could take. The case study will focus explicitly on dictionary users and will apply a theoretical framework based mainly on Tarp's (2009) theory of lexicographical functions (discussed in section 4.1.1 - Theory of lexicographical functions). One of the main arguments this thesis will make is that folk definitions (defined here loosely as informal explanations of word meaning by native speakers) provide an appropriate starting point for the process of creating and evaluating signed definitions.

\subsection{Motivation for the thesis}

My personal interest in this area stems from my involvement in two different sign language dictionary projects. As a Masters student in the early 1990s, I had the opportunity to observe at close range the final stages of production of the pioneering Dictionary of British Sign Language / English (Brien, 1992). Although my role in this project was purely administrative, the long hours of typing up dictionary entries and manually ordering transcriptions gave me an insight into the immense challenges of representing the signed lexicon in a static, printbased format. Moreover, the project instilled in me the values of a descriptive linguistic approach centred on, and with direct involvement of, Deaf sign language users. These values are reflected in the objectives of the dictionary editorial team:

"1. that the signs contained in the Dictionary of British Sign Language / English (DBSL/E) should be BSL signs, i.e. signs identified by Deaf informants as signs they themselves, or other Deaf people, used when communicating in BSL;

2. that the entries should be organised on linguistic principles, appropriate to a signed language;

3. that a guide to the meaning(s) of the signs (and thereby a guide to appropriate usage) be provided;

4. that the DBSL/E should be of use to both Deaf users of BSL and people wishing to learn the language."

(Brien \& Turner, 1994, p. 394)

These four objectives have further relevance to the current thesis. Firstly, they demonstrate the envisaged multiple functions of sign language dictionaries, intended to serve both as tools for language description and documentation, and as practical reference works.

Secondly, they show the intention that a single sign language dictionary would be used by both L1 (first language) users of a sign language and L2 (second or other language) learners. 
This goal of serving multiple user groups, typical of most sign language dictionaries to date, reflects the realities of making a dictionary for a minority language which may lack governmental or institutional support and which may not have a sufficiently large market to make it commercially viable.

Thirdly, objective 3. shows that a 'guide to meaning' was considered an essential part of the Dictionary of British Sign Language/English, and that information on both the semantics and usage of signs should be shown. In spite of the fact that the descriptive part of the dictionary was not given the title 'definitions'-Brien and Turner (1994) reasoned that this designation was not warranted since there was not always sufficient evidence of usage to be 'definitive' about the meanings of the signs - the function of the 'Guide to Meaning' was equivalent to that of a dictionary definition, i.e. helping users to understand the meaning(s) and use of signs. The constraints of the print format meant that this information had to be shown in English, rather than in BSL. Significantly, the chosen format of the 'Guide to Meaning' consisted of a series of full-sentence definitions, adapted with permission from the then recently published Collins COBUILD English Language Dictionary (Sinclair, 1987 a). Brien and Turner's rationale for choosing full-sentence definitions as clear, concise and comprehensible statements about meaning that were particularly suited to language learners prompted my first interest in this more informal format as a model for signed definitions.

Nearly twenty-five years later, I started work as the Database Manager on a three-year project to create a multi-media, online resource based on the contents of the existing print Dictionary of New Zealand Sign Language (Kennedy, Arnold, Fahey, \& Moskovitz, 1997) and Concise Dictionary of New Zealand Sign Language (Kennedy, McKee, Arnold, Dugdale, Fahey, \& Moskovitz, 2002). In the process of populating a new custom-made database (created by the Technical Editor), all previous dictionary entries were reviewed and updated by the editorial team and a number of new records were added from data gathered and validated in the years since the publication of the print dictionaries. The Online Dictionary of New Zealand Sign Language (ODNZSL) (McKee, McKee, Pivac Alexander, Pivac, \& Vale, 2011) was launched in 2011.

The comprehensive project description by the editors (McKee \& McKee, 2013) clearly outlines the advantages of the online format over the previous print-based medium. The ODNZSL includes videos showing how each sign is produced, is searchable in both language directions, and includes examples of use that were informed by a digital corpus of signed 
texts. In other respects, the challenges and objectives of sign language lexicography have remained the same. The ODNZSL, just as the print NZSL dictionaries and the Dictionary of $B S L /$ English before it, is a descriptive multifunctional dictionary with limited resources that has to meet the needs of a wide range of user groups.

The ODNZSL and other online sign language dictionaries exist in a climate of increasing interest in learning sign languages and expansion of the domains in which sign languages are used, while at the same time there are indications that the number of people who use a sign language as their first language is diminishing. The factors that now place some sign languages at risk of becoming endangered languages will be described further in section 2.1.3 - Threats to the vitality of sign languages. In this context, it is even more important than before to document sign languages as fully as possible, in order to preserve and maintain the linguistic and cultural perspectives of Deaf sign language users. Schermer (2006) argued that monolingual sign language dictionaries are a necessary development in this respect, both "to do full justice to the richness of the lexicon of sign languages" (p. 322) and as the descriptive basis of bilingual dictionaries. However, given the resource constraints noted, it remains unlikely that purely monolingual (general) sign language dictionaries will see the light of day. A more practical avenue of research would therefore be to explore ways of incorporating additional signed descriptions of the sign language lexicon into the existing multifunctional, bilingual format.

The suggestion to include definitions in electronic sign language dictionaries is not new. Some of the recent literature on sign language lexicography has suggested that definitions would not only provide better description of the signed lexicon for documentation purposes, but that they would also be of practical use for language learners as dictionary users. For example, Kristoffersen and Troelsgård (2012) stated that definitions would be “... an obvious enhancement of comprehensive sign language dictionaries..." (p. 312), offering an improvement in semantic description from the current listing of one or more equivalents in the spoken language and potentially helping users to locate the right word or sign both in language comprehension and production; and McKee and McKee (2013) found that there would be scope for including encyclopaedic-type definitions for culturally rich lexical items, "especially for non-native users wanting to understand why a word exists and why a speaker would choose to use it in a context-in effect, providing ethnographic reference." However, there has so far been a lack of empirical evidence to establish whether dictionary users would indeed benefit from signed definitions. Moreover, the factors that have so far prevented the 
inclusion of definitions should be examined to find out whether it would be worthwhile to invest effort in their development in the current context.

Kristoffersen and Troelsgård's (2012) observation that no usability studies of current sign language dictionaries had been carried out, and their suggestion of Tarp's (2009) lexicographical function theory as a framework for investigating the format of sign language dictionaries, prompted my decision to carry out a user-focused case study of a current dictionary. My continued involvement in the maintenance of the database and website of the ODNZSL, as well as its features including bidirectional search facilities and signed example sentences, made this dictionary an obvious choice as the subject for a case study. My role as Database Manager afforded me access to anonymous log file data showing interactions with the ODNZSL website and I was of course very familiar with its contents. I have benefited greatly from discussions with the ODNZSL editorial team during all phases of its creation and upkeep. However, the research presented in this thesis and the application of a theoretical lexicographical framework are my own and were carried out independently.

Throughout this research, I have been conscious of my position as a hearing, non-native sign language user in this field. My proposal of eliciting folk definitions as the basis for creating signed dictionary definitions was primarily motivated by my desire to minimise my own influence on the form of signed definitions and to foreground Deaf sign language users' perspectives. The Deaf participants in my research have also had an active role in the evaluation of these definitions and in the creation of the pilot dictionary definitions.

\subsection{Aims and significance of the thesis}

The overall aim of the thesis is to explore the question: should signed definitions be incorporated into sign language dictionaries?

From the preceding short overview of the context in which sign language dictionaries are developed and used, it will be clear that a number of contributory issues will need to be addressed in order to answer the main research question.

- First of all, in view of the importance of a user-focused perspective in lexicography, we should ask what purposes signed definitions would serve and for whom. Although sign language dictionaries claim to be useful to a wide range of user groups, there is a lack of empirical evidence on actual dictionary use. Fundamental questions that should be asked first are therefore: who are the users of sign language dictionaries? What situations 
prompt dictionary use? How are these dictionaries used? Do users have particular problems or issues in using sign language dictionaries?

- Secondly, the current provision of information in sign language dictionaries needs to be evaluated in order to establish in which specific situations and for which lexical items this information may be inadequate.

- Thirdly, we need to consider whether it is possible to create definitions that are capable of meeting the needs of sign language dictionary users while at the same time supporting the broader ideological aims of sign language dictionaries.

- Finally, since the thesis argues that folk definitions should form the basis of signed dictionary definitions, the proposed usefulness of folk definitions as a model needs to be evaluated in practice.

By examining the research question from these various theoretical and practical angles, the thesis provides much-needed empirical data in a number of areas that have so far received little attention. The outcomes of this research will help sign language lexicographers to direct the limited resources available for dictionary making towards those areas where they will be most effective. This in turn may lead to sign language dictionaries that are better able to meet the needs of their users, and that therefore ultimately contribute to the promotion and maintenance of sign languages.

\subsection{Overview of the thesis}

This introductory chapter has set out the rationale for investigating the potential role of signed definitions in sign language dictionaries.

Chapter 2 - Sign languages and the sign language lexicon outlines the sociolinguistic context of sign languages and the nature of the sign language lexicon to identify potential obstacles to the inclusion of signed definitions.

Chapter 3 - Sign language lexicography reviews the history, purposes and format of sign language dictionaries and describes developments in electronic lexicography that make it possible to add new elements to the dictionary entry in video form.

The remainder of the thesis consists of four successive studies. Each of these studies is treated in a separate chapter that contains a distinct set of research questions, a review of the literature and methodology relevant to the particular study and a description of the 
method(s) used. Each chapter then reports on the results of the study, discusses the findings and limitations and relates these to the overall research question.

Chapter 4 - A user study of an online sign language dictionary introduces the Online Dictionary of New Zealand Sign Language (ODNZSL) as the case study for this thesis and describes an empirical user study of this dictionary. The results of this study lead to an analysis of the potential user needs for signed definitions.

Chapter 5 - Evaluating the user need for definitions focuses on the particular user needs of hearing, intermediate L2 learners of a sign language. An analysis of the current content of the ODNZSL, in particular English equivalents and signed examples, will be used to determine for which categories of lexical items the current description may be insufficient or misleading. The chapter then reports on the second study: a computer-based error correction test assessing whether the identified categories were in fact problematic for learners in comprehension or production.

Chapter 6 - Folk definitions describes the third study, in which a selection of signs that were identified as problematic in the previous study were used to elicit folk definitions from Deaf users of New Zealand Sign Language. An in-depth analysis of the content and form of the folk definitions will be used to support the proposal that signed dictionary definitions should be modelled on the folk definition format.

Chapter 7 - From folk definition to dictionary definition reports on the fourth study, which consisted of the creation of a small pilot set of signed dictionary definitions and a number of evaluations of their effectiveness, including a repeat of the error correction test comparing test items with and without definitions.

Finally, Chapter 8 - Conclusion summarises and synthesises the findings of the four studies, provides a qualified answer to the question whether signed definitions should be added to sign language dictionaries, and discusses the implications of the thesis for sign language lexicography. 


\section{Chapter 2: Sign languages and the sign language lexicon}

\section{Introduction}

This thesis asks whether signed definitions can make a contribution to sign language dictionaries. In order to answer this question, it is first of all necessary to explore why sign language dictionaries to date have rarely included definitions of any kind. There are broad sociological and linguistic factors that ultimately have an influence on the format and content of sign language dictionaries. This chapter therefore begins with a description of the sociolinguistic context of sign languages, focusing on the origins of Deaf communities and the position of sign languages as minority languages in constant contact with the surrounding majority language and culture. The current and future status of sign languages is partly determined by tensions between increasing formal recognition and associated expanding contexts of use, juxtaposed with threats to their vitality because of changing educational and medical policies that affect the deaf population.

Next, the lexicon of sign languages will be described, outlining different approaches to sign linguistics research and describing structural aspects that may be unique to the visualgestural modality and that are challenging for sign language lexicographers. The question of what constitutes a lexeme in a sign language will also briefly be addressed. The sign language lexicon will be shown to have a relatively small core vocabulary of established lexemes, with a much larger non-core or 'productive' lexicon and several non-native influences.

\subsection{The sociolinguistic context}

\subsubsection{Origins of natural sign languages}

Natural sign languages originate from within communities of Deaf people. There is evidence of signing being used by deaf people throughout history. Although it is highly likely that individual deaf people will develop some kind of gesture system to communicate with other people in their environment (Frishberg, 1987), for natural sign languages to evolve requires sufficient numbers of people in a community to use signing as the main form of communication.

There are a small number of communities where the prevalence of inter-generational deafness is much higher than in the general population (for example, through hereditary 
deafness) and where sign languages have developed that are used by deaf and hearing people alike. Documented cases of such sign language communities include Martha's Vineyard in the USA (Groce, 1985) and village communities in Jamaica, India, Turkey, Thailand and Bali (Zeshan \& De Vos, 2012).

With the exception of communities such as these, the incidence of deafness in the general population is generally low. The majority of deaf children (90-95\%) are born in hearing families (Mitchell \& Karchmer, 2004) where a spoken language is the main form of communication. Sign languages are therefore not usually passed on from parent to child. Instead, many deaf children are only introduced to sign languages at a later age when they come into contact with Deaf adults or with deaf peers. Traditionally, residential Deaf schools have therefore been sites for transmission of sign languages, and places around which Deaf communities were established (Ladd, 2003).

\subsubsection{The status of sign languages}

Although educational institutions provided an impetus for the formation of Deaf communities, predominant educational philosophies have actively tried to counter the use of sign languages, instead advocating the use of methods that emphasised speech and the amplification of sounds in order for deaf children to acquire the spoken language of the surrounding community. This philosophy, known as 'oralism' or 'the oral method', was especially influential from 1880, when a congress of (almost exclusively hearing) educators passed a resolution banning the use of sign language in education (Lane, 1984). The effects of this ban persisted in Europe and the United States (and in Australia and New Zealand) for most of the next 100 years. Although sign languages continued to be used outside the classroom and in Deaf clubs, the lack of institutional and societal support restricted their use in official or public domains.

Recognition of the linguistic properties of sign languages by Tervoort (1953) in the Netherlands and Stokoe (1960) in the United States led to a gradual reversal of this situation. In many countries, the 1970 and 1980 s were marked by Deaf communities' increasing selfawareness and sense of pride in the use of their sign language. Discourses emerged that described the Deaf community as a cultural and linguistic minority group (Padden, 1980; Lane, 1995). This period also saw an acceptance of sign languages in wider domains, including the re-introduction of signing in education and in some cases, public broadcasting making sign languages more visible to the majority hearing communities. The increased 
status of sign languages has led to their legal recognition in some countries (Reffell \& Locker McKee, 2009; Murray, 2015). However, such recognition has mainly been symbolic and has not been accompanied by resources or systems that would support the maintenance and promotion of the language (McKee \& Manning, 2015; Murray, 2015).

\subsubsection{Threats to the vitality of sign languages}

The expansion and increased visibility of sign languages in the public domain in recent decades is offset by noted decreases in the number of Deaf sign language users (Johnston, 2006). The closure of many deaf schools as a result of educational policies favouring mainstream school placements for deaf children has reduced traditional transmission opportunities for sign languages. The discourses that construct Deaf people as forming a linguistic minority have not taken root in the dominant hearing society, where deafness is still often considered to be a purely medical condition and a disability (Lane, 1995). As a result, there is a continued emphasis on medical interventions and hearing aid technology as ways of promoting the acquisition of the spoken language by deaf children. Since the use of sign languages, if not actively discouraged, is still not promoted or supported, and signing populations are decreasing, a number of sign languages have now been identified as at risk or endangered (McKee, 2016; Bickford, Lewis, \& Simons, 2015; Murray, 2015).

It is estimated that sign languages are the first or primary language of around 70 million people worldwide (World Federation of the Deaf, n.d.), although such estimates are difficult to make given the dispersion of deaf people and varying degrees of record keeping. In New Zealand, estimates of the number of sign language users vary from 6,900 (Greville, 2005) to 9,00o (Deaf Aotearoa New Zealand, 2011) primary users, although the group of people with a basic knowledge, defined as able to have a conversation "about a lot of everyday things" in NZSL is considerably larger at around 20,244 people in the most recent New Zealand Census (Statistics New Zealand, 2013).

In common with other minority languages that have been used in limited domains, Deaf communities have traditionally communicated face-to-face. Sign languages have remained unwritten languages: although a writing system called 'SignWriting' has been promoted with some success since its creation in 1974 (Sutton, 2002), it has never gained widespread use. Video recording has taken over some functions of written texts, but there is no sign language print culture. 


\subsubsection{Language contact between signed and spoken languages}

Due to the factors described above, sign languages, although unrelated in both modality and structure to the dominant spoken languages that surround them, are subject to constant influences arising from close language contact. Sign language users are rarely monolinguals and will usually be familiar with many aspects of the spoken language, even though this language may have been acquired only partially and imperfectly. Natural language contact phenomena such as code-switching, code-mixing and borrowing (e.g. Battison, 1978; Lucas \& Valli, 1990) are therefore common. These phenomena will be described in more detail in section 2.2.5 - The non-native lexicon.

In addition to these natural processes, attempts have been made at various times to introduce visual-gestural representations of the spoken / written language in deaf education, with the intention of teaching deaf children to speak and learn the grammar of the spoken language. Some systems such as Cued Speech use different hand configurations to represent spoken language phonemes (National Cued Speech Association, n.d.). Other systems use lexical signs (either created or borrowed from existing natural sign languages) to accompany the spoken words in a sentence. In these systems, signs are typically combined sequentially to follow the word order of the spoken language. Some systems also add specific hand forms as 'affixes' to represent spoken language morphemes such as a plural or possessive -s in English, or include signs to represent words perceived as 'missing' in sign language, such as the copula 'to be' (see e.g. Ahlgren, 1986). In addition, manual alphabets were historically in use in various hearing communities, including as a communication method for certain religious orders, and these alphabets were introduced in deaf education at various times (Padden \& Gunsauls, 2003).

The results of these contact phenomena are found in both situational and social variation of sign languages. Signed interactions in the presence of hearing individuals or in formal settings are likely to elicit more contact forms (e.g. Lucas \& Valli, 1990). Certain individuals are also more likely to use spoken language-influenced forms because of their educational background and their age of socialisation into the Deaf community.

\subsubsection{Sociolinguistic variation}

Sociolinguistic studies of sign languages have identified situational and social variation at phonological, morphological, syntactic and lexical levels (Lucas, Bailey, Valli, Rose \& Wulf, 2001; Schembri \& Johnston, 2004; McKee \& McKee, 2011). Common social variables of region, 
age, gender and ethnicity have been found to play a role in sign language variation; however, underlying these social variables there are wider 'macro' factors around the formation of Deaf communities. For example, it has already been mentioned that Deaf communities tended to gather in particular locations because of the presence of a Deaf school, and that educational policies and societal acceptance of sign languages have varied over time and in different countries.

It has become usual to name sign languages after the country in which they are used-for example, American Sign Language, New Zealand Sign Language, or Sign Language of the Netherlands. Naming a sign language is a powerful symbolic act validating its linguistic status. Naming sign languages after their country of use also highlights the fact that there is no universal sign language, and acknowledges the rights of Deaf people as citizens of their country and users of a minority language. However, there is a risk that the use of a national sign language name, paired with a drive for language standardisation, may suppress local variation (Branson \& Miller, 1997).

In fact, national sign languages show a great deal of internal variation. For instance, McKee and McKee (2011) found considerable lexical variation in NZSL, which they ascribed in part to the relatively recent expansion of NZSL into new domains and the absence of standardising influences such as the use of sign language in public media, in education, or in written form.

\subsubsection{Effect of age of acquisition}

There is ample evidence that when sign languages are acquired from infancy, for example by children growing up in families where both parents are sign language users, acquisition follows the same stages and milestones as for the acquisition of a spoken first language (see e.g. Woll, 1998; Petitto, 2000; van den Bogaerde, 2000). Where sign languages are acquired later in life, certain aspects may not be completely acquired. (Woll, 1998). Late acquisition particularly affects sentence-level processing and the comprehension of multi-morphemic signs (Mayberry \& Eichen, 1991).

\subsection{Linguistic structure and the lexicon}

\subsubsection{Approaches to the description of sign languages}

In the description of the sign language lexicon that follows, it is important to bear in mind that the recognition that sign languages are fully-fledged, natural human languages is still fairly recent. The prevalent opinion until the middle of the last century was that signs were 
simple, holistic gestures miming real-life actions and objects. These perceptions are still widely held amongst the lay public and they give rise to common misperceptions of sign languages as either created systems that represent spoken language on the hands, or alternatively as simple concrete depictions incapable of abstract expression, and the related assumption that sign language is a universal language.

The seminal work of Stokoe (1960) identified the structured formation of signs from a finite set of components. This analysis opened the way for further research on linguistic aspects of sign languages. In the relatively short time since Stokoe's groundbreaking study, sign linguistics has developed as a discipline that draws on all branches of general linguistics.

A distinction can be drawn between two ways in which sign linguistics has positioned itself with respect to general linguistic models of language structure and use. These two approaches were respectively referred to by Karlsson (1984) as 'oral language compatibility' and 'sign language differential' views. Oral language compatibility approaches emphasise the similarities between sign language structures and known models of spoken languages, for example by applying existing terminology to phenomena found in sign languages. This approach was particularly common in several influential studies in the 1970 and particularly the 1980 os (e.g. Supalla \& Newport, 1978; Wilbur, 1979; Klima \& Bellugi, 1979; Liddell, 1980; Petitto, 1987; Kyle \& Woll, 1987; Collins-Ahlgren, 1989) when there was considerable pressure to prove that sign languages met all the criteria of spoken languages and yet were autonomous systems. Subjects that seemed to contradict this autonomy, such as similarities to gesture, iconicity, or mouthing patterns influenced by spoken words, were avoided or deemphasised in this period (Vermeerbergen, 2006). The sign language differential view, also found in very early research, became more evident in later years. This approach focuses on the properties that are unique to sign languages due to the different organisational possibilities of the visual-gestural modality (a notable example is Cuxac, 1996; see also e.g. Johnston \& Schembri, 1999). Vermeerbergen (2006) noted that the oral language compatibility approach has been dominant until recently.

The presence of these two distinct approaches has led to at times vastly different descriptions of the lexicon of sign languages, for example in the areas of determining word classes and inflectional or derivational patterns. Furthermore, sign linguistics is also subject to shifting models in general linguistic theory. For example, many of the influential studies in the 1970s and 1980 os cited above drew on generative linguistics and analysed sign languages accordingly 
as highly inflected languages (e.g. Supalla, 1982, 1986; Padden, 1983); whereas other researchers have taken a cognitive linguistics approach focusing on conceptual models in their descriptions (e.g. Van Hoek, 1992; Liddell, 2003; Leeson \& Saeed, 2012). Features such as gesture and iconicity have been found to play a greater part in spoken languages than was previously thought (e.g. Kendon, 2004; Perniss, Thompson \& Vigliocco, 2010), leading to a reevaluation of the question whether the visual-gestural modality leads to unique features in sign languages, or whether certain language features are universal and sign languages and spoken languages share the same 'deep’ structures (e.g. Sandler \& Lillo-Martin, 2006).

The influence of these contrasting models on perceptions of the sign language lexicon is also evident in sign language dictionaries. A crucial period for sign language lexicography was the 1990s, when many linguistically informed descriptive sign language dictionaries saw the light (e.g. Johnston, 1989 a; Suwanarat, Ratanasint, Rungsrithong, Anderson, \& Wrigley, 1990; Brien, 1992; Kennedy et al., 1997). These dictionaries showed attempts to represent the unique properties of signs in some respects, for example ordering dictionary entries by phonological features of the sign and including 'proto-definitions' to draw attention to complex lexical sign constructions (Brien \& Turner, 1994). Other aspects of these dictionaries, however, were influenced by existing spoken language models: for example, signs in $A$ Dictionary of New Zealand Sign Language (Kennedy et al., 1997) were assigned to particular word classes even though multi-functionality of signs is common and there is indeterminate evidence for these classifications based on sign morphology (Meir, 2012).

\subsubsection{The sign language lexicon}

The main task of lexicography is to analyse and describe the lexicon of a language. The question of what constitutes a word, or more accurately a lexeme, has therefore been a central issue for dictionary-makers. For sign languages, the most comprehensive treatment of this question can be found in Johnston and Schembri (1999). The initial distinction made in this article is between non-linguistic gesture and linguistic signs, with the latter having a more consistent form, an internal structure, often a componential meaning, and being able to be combined in sentences. In the first instance, Johnston and Schembri therefore defined a sign as

"a relatively stable, identifiable visual-gestural act with an associated meaning which is reproduced with consistency by native signers and for which, consequently, particular agreed values can be given for handshape, orientation, location and movement (including lack of movement)." 
Within this definition Johnston and Schembri identified a smaller set of lexemes, which have an identifiable citation form and a context-independent meaning. They reviewed phonological, morphological and semantic criteria that can be used to distinguish between lexemes and other signs and reached the conclusion that in comparison with spoken languages, the collection of established sign lexemes is considerably smaller, typically numbering only around 3,00o to 4,ooo lexemes for the sign languages investigated. The larger part of the sign language lexicon therefore consists of other kinds of signs-mainly depicting signs and pointing signs - that can vary considerably in form and are dependent on the context for their meaning. This part of the lexicon is referred to as the 'non-core' (Brentari \& Padden, 2001) or 'productive' lexicon (Brennan, 1990).

A further distinction has been made between the 'native' lexicon, consisting of both core and non-core signs used by Deaf people, and 'non-native' vocabulary (Brentari \& Padden, 2001). Non-native signs are derived from contact with the majority spoken language, for example through spelling out written words through a manual alphabet (a process called 'fingerspelling') or incorporating handshapes representing individual letters into existing signs; through mouthing words from the spoken language while producing a manual sign; or through loan translations. Johnston and Schembri (2007) also consider borrowings from other sign languages, arising from increasing international contact between Deaf communities, to be a part of the non-native lexicon.

The non-core and non-native lexicon will be discussed in more detail below. First, a brief account will be given of the structure of signs and four modality-specific factors that are relevant in discussing the nature of signs.

\subsubsection{Modality and structure}

Sign languages are languages in the visual-gestural modality, as opposed to the auditory-oral modality employed by other human languages. Stokoe (1960) found that signs used by American Deaf people consisted of several parameters, including a hand configuration (usually referred to as a 'handshape' in the sign linguistic literature), a location on the body or in front of the body, and a movement. Stokoe coined the term 'chereme' for these components as an alternative for the 'phonemes' of spoken languages, although this term was not widely adopted and has fallen into disuse. One difference between cheremes and phonemes is that cheremes are combined more or less simultaneously whereas sequential patterns are predominant in the phonology of spoken languages. 


\subsubsection{Simultaneity}

Sign languages show simultaneity of structuring at all linguistic levels. Simultaneity in sign languages is possible because of the availability of a three-dimensional space in which signs are produced, and because there are multiple articulators: both hands, facial expressions including movements of the eyes and mouth, and (upper) body movements can all form part of the production of a signed utterance. Sandler and Lillo-Martin (2006) discuss three reasons for the prevalence of simultaneous structures in sign languages. Firstly, a tendency towards iconicity (see section 2.2.3.3 - Iconicity) combined with the availability of multiple articulators means that real-life events, often involving multiple actors and objects simultaneously present, will be represented as directly as possible. In other words: "The reason that sign languages express simultaneous events through simultaneous form may be simple: they do so because they can." (p. 491).

Secondly, the hands as articulators lend themselves to representing the form of objects and actors, while at the same time their movement through the signing space can show the actions performed by and with these objects and actors.

Finally, the production of a sign is typically slower than a spoken word and there is evidence for processing restraints and a shorter working memory span for signed information (e.g. Emmorey, 2002). Simultaneity allows for similar amounts of information to be conveyed in sign languages in spite of the slower lexical production rate (Bellugi \& Fischer, 1972; Klima \& Bellugi, 1979).

At the phonological level, Stokoe (1960) already noted that unlike the sequential production of spoken language phonemes, cheremes were produced simultaneously. Later studies also focused on simultaneity at morphological, syntactic and discourse levels (e.g. Miller, 1994; Engberg-Pedersen, 1994; Liddell, 2003). Vermeerbergen, Leeson, and Crasborn (2007) categorised simultaneous patterns into three different types:

- Manual: where each hand articulates different information;

- Manual-oral: where the mouth produces morphological, lexical or syntactic information that supplements the signs produced by the hands;

- Other: where information is conveyed simultaneously by various body parts other than the mouth (for example, eye gaze or body leans). 


\subsubsection{The use of space}

One of the phonological features identified by Stokoe (1960) is the location where a sign is produced. In Stokoe's analysis, the space in front of the signer is called the 'neutral space'. The neutral space is contrasted with all other places on a signer's body where a sign can be produced, but individual points in this neutral space are not contrastive (see e.g. Stokoe, Casterline, \& Croneberg, 1965). At the morphosyntactic and discourse levels, however, individual points in the signing space become meaningful when they are used to establish a relationship between referents and their location (e.g. Sandler \& Lillo-Martin, 2006). Referents can be associated with a location in the signing space by placing signs in that location, pointing towards it, or by directing eye gaze towards it. Spatial reference underlies pronominal and verb agreement systems as well as providing a means of representing spatial relationships between objects and their movements through physical space, through productive sign forms. Some researchers distinguish between 'syntactic' and 'topographic' use of space (e.g. Klima \& Bellugi, 1979; Sandler \& Lillo-Martin, 2006) whereas others (e.g. Van Hoek, 1992; Engberg-Pedersen, 1993; Liddell, 2003) have argued that these functions are integrated and that both are based on a conceptual representation of referents in 'real' physical space.

\subsubsection{Iconicity}

A key difference between spoken and signed languages is that the latter show a much higher proportion of iconicity (the resemblance of the form of a word or sign to its meaning). Although Perniss et al. (2010) stress that iconicity is more prevalent in spoken languages than previously assumed, especially in languages outside of the Indo-European family, the more usual relationship between the form of a spoken word and its meaning is arbitrary. In sign languages the reverse holds true: although arbitrary form-meaning relationships do occur, sign forms that are at least partly motivated are by far the most frequent. Current research ascribes this frequent iconicity to the idea that the visual modality lends itself to more direct representation of real-life actions (the hands and body can carry out imitations of the way they would move or interact with objects in the real world) and that perhaps, visual iconicity is more readily available than resemblances between sounds and the real world (Demey, van Herreweghe, \& Vermeerbergen, 2008; Perniss et al., 2010).

At first glance the high level of iconicity reinforces the lay perception of signs as simple mimetic actions. However, rather than being holistic imitations, iconic signs appear to comply with the same constraints on internal sign structure as abstract signs. For example, 
Klima and Bellugi (1979) reported that there were differences in the handshapes that were permissible in the construction of signs in ASL and Chinese Sign Language, and that these constraints applied to iconic as well as arbitrary signs.

Researchers are not fully agreed on the precise interactions between iconicity and the internal structure of signs (Zwitserlood, 2012). However, it is clear that the sub-lexical elements that were previously described as sign language 'phonemes' (hand configuration or handshape, location, and movement) also frequently have a direct form-meaning relationship. In other words, in many signs these basic parameters themselves act as morphemes contributing meaning to the sign construction in which they are used. For example, the movement of the NZSL sign SLOW is indeed slower than that of the sign FAST. Because of such iconic relationships, the traditional distinction between the phonological and morphological level is blurred in sign languages. One suggestion has been to reinstate the term 'chereme' or to allow other terminology specific to sign language 'phonemes' to develop (Johnston \& Schembri, 1999); however, this suggestion has not been widely taken up and instead many sign language researchers currently refer to 'phonomorphemes' (e.g. Demey et al., 2008).

Particular attention has been paid to the form-meaning relationships of the hand configuration parameter, used to "represent entities by denoting salient characteristics" (Zwitserlood, 2012), in polycomponential sign constructions. This will be further discussed in section 2.2.4 - The non-core lexicon.

Taub's (2001) cognitive linguistic model provides a possible account of the process by which signs are constructed using available (phonological) components to represent selected features of a real-life referent (see figure 2.1).
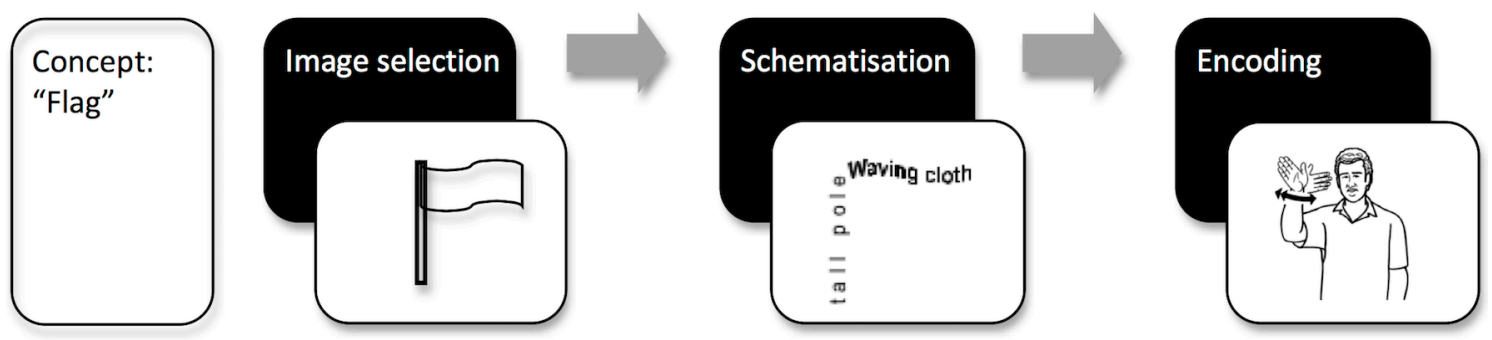

Figure 2.1: Iconicity—selection, schematisation and encoding (following Taub, 2001)

Taub's model also shows why the same concept may be represented differently in different sign languages (or even within the same sign language). Figure 2.2 shows entries from the Online Dictionary of New Zealand Sign Language (McKee et al., 2011) that represent an 
original NZSL sign (1) and a later borrowing from ASL (2) for the concepts of 'tree' and 'house'. It can be seen that these signs are all iconic but are based on different selected features of the concept. TREE 1 is based on an outline of the foliage whereas TREE 2 shows the whole object shape (trunk and branches); HOUSE 1 outlines both roof and walls but HOUSE 2 shows only the roof.

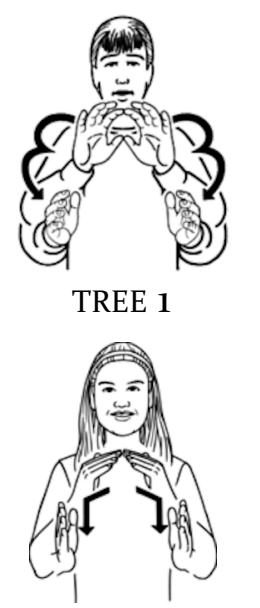

HOUSE 1

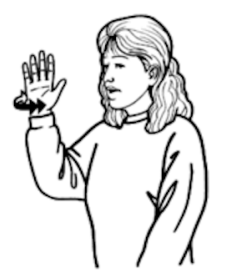

TREE $\mathbf{2}$

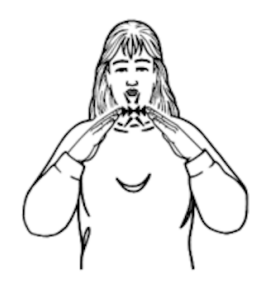

HOUSE 2

Figure 2.2: Iconic signs derived from schematisation of different features

Although iconicity has been shown to play a large role in sign languages, this does not necessarily mean that all motivated signs are transparent. Johnston (1989 b) distinguished between transparent, translucent, obscure and opaque signs and stated that even though many signs may originally have been motivated, the selected iconic features may no longer be recognised by later generations of signers. Sutton-Spence and Woll (1999), amongst others, argued that young children may not perceive iconic relationships that are transparent to adults: for example, if children have grown up in the city they may not understand that the sign MILK draws on the action of milking a cow. Boyes Braem, Pizzuto, Volterra, Schulmeister, and Reinitzer (2002) reported on a study of hearing and Deaf people's interpretations of Italian Sign Language (LIS) signs, showing that the meaning of deictic and pantomimic representations was more easily interpreted than the meaning of signs with a metonymic or metaphorical relationship. They also argued that cultural background may influence the schematisation of features: for example, although some signs for WOMAN may be based on universal characteristics such as body shape or an outline of the breast, other sign languages may select different culturally salient features, such as lipstick, earrings, or long hair.

First language acquisition of sign languages does not seem to be affected by iconicity, with children acquiring iconic signs no earlier than abstract signs. (e.g. Newport \& Meier, 1985). In contrast, there is evidence that adult L2 learners do use iconicity as an aid to learning signs 
(Campbell, Martin, \& White, 1992), although Mayberry (2006) states that this strategy is abandoned after learners know the phonological structure of signs. Smith, Tsimpli, Morgan, and Woll (2011) mention, however, that it is more usual for learners to discover the motivation for a sign's form after they have already been given a spoken language equivalent for the sign. Iconic signs do seem to be easier to remember for adult L2 learners, according to Johnston and Schembri (2007); it may therefore be the case that iconicity functions as a memory aid by establishing a conscious form-meaning link, facilitated by the learner's L1.

\subsubsection{Metonymy and metaphor}

Figure 2.2 in the previous section showed how signs may select and encode different salient elements. Whereas some signs depict a referent or action in its entirety, it is also very common for signs to draw on metonymy or synecdoche (part for whole). In the sign HOUSE, for example, the sign depicts only the roof; similarly, a conventional way to refer to the actions of a person is by the use of two extended fingers to represent the person's legs, as can be seen in the NZSL signs WALK, STAND, and JUMP (see figure 2.3).

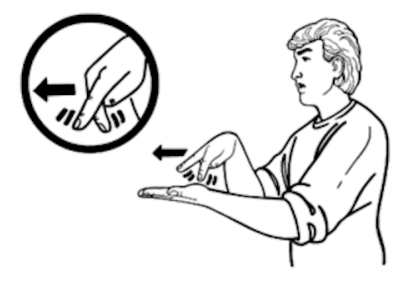

WALK

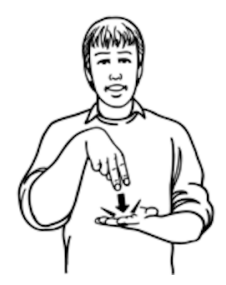

STAND

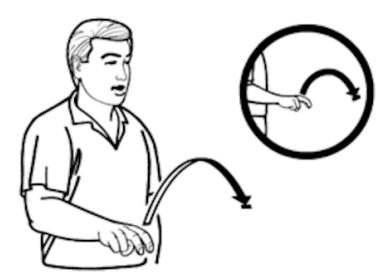

JUMP

Figure 2.3: NZSL signs using the fingers to represent a person's legs

Examples of figurative extension of this part-for-whole relationship in signs are found frequently in place and country names: for example, GERMANY refers to the spike on a traditional German helmet, PARIS depicts the Eiffel Tower, and SCOTLAND is shown by the action of compressing bagpipes.

Several researchers (notably Mary Brennan (1990, 1992, 2005) for BSL and Phyllis Wilcox (2000, 2005) for ASL) have pointed out the extensive use of metaphor in the creation of signs. Just as is the case for spoken languages (as described by Lakoff \& Johnson, 1980), metaphors in sign languages describe abstract ideas by using vocabulary from a concrete source domain. Lakoff and Johnson (1980) give the example of an equation of the abstract concept of 'time' with the concrete manipulation of 'money', thus allowing for expressions such as 'spending time with someone' or 'wasting your time'. In sign languages, according to Taub (2001), a 'double mapping' takes place to create metaphorical signs. The first mapping is the previously 
mentioned selection of meaningful sign components to represent a concrete object or action. This object or action is then used in a figurative sense to describe the abstract idea. For example, the NZSL sign REMEMBER (figure 2.4) shows the iconic action of holding an object being applied to 'holding' an idea inside the mind.

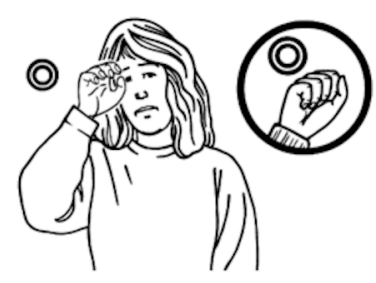

Figure 2.4: Double mapping in the NZSL sign REMEMBER

Brennan (1990) identified a number of prototypical metaphors in BSL and showed how each of the sign parameters can exhibit the double mapping mentioned by Taub (2001) and be a meaningful part of metaphorical sign constructions. For example, in the sign illustrated above, the handshape shows an abstract idea being 'held', whereas the location on the forehead represents the mind as a container into which thoughts can be placed or held. Relative locations in the signing space can also be meaningful, for example in the prototypical opposition metaphor, where the two hands are placed opposite each other in the signing space to express concepts such as 'arguing' or 'war'. In the definite vs. indefinite metaphors, it is the movement that exhibits double mapping: the movement in signs such as CERTAIN is firm and fast, whereas signs such as APPROXIMATE or UNSURE have a wavering or circular motion.

\subsubsection{The non-core lexicon}

\subsubsection{Depicting signs or polycomponential signs}

The previous sections have outlined how signs are constructed from different simultaneously produced components and that the form of signs is often motivated, either by a direct relationship between the form and meaning of the sign or by a metaphorical extension of an iconic image. Although the sign in its entirety can resemble an object or action, it has been found that sub-lexical components (handshape, location, and movement) are also frequently individually meaningful. For example, in the earlier example given in figure 2.3, the hand configuration with two extended fingers represents the legs; the movement describes the action; and the location where the sign is produced may also be a representation of real or metaphorical locations. Non-manual features may be included to show the manner of the action, for example whether someone is walking with ease or with effort. 
Signs such as these are frequently referred to as 'classifier verbs' or 'classifiers' (see Schembri, 2003 for a list of variations on this terminology). This terminology originated in early research on ASL (e.g. Frishberg, 1975), through a perceived parallel with certain spoken languages such as Navajo, in which some morphemes have the function of marking referents out as belonging to a certain class. The handshape in sign languages is said to fulfill a similar function, representing classes of entities "by denoting salient characteristics" (Zwitserlood, 2012). The handshape can represent the referent as a whole entity, show how the referent is handled, or outline the referent's size and shape (Supalla, 1986).

There is considerable disagreement about the appropriateness of the 'classifier' term as applied to sign languages, partly because of changing definitions of classifiers in spoken language linguistics, and partly because of a move away from the oral compatibility approach that motivated the appropriation of spoken language linguistic terms to label sign language phenomena (Schembri, 2003). In addition, the status of the subcomponents of these signs as morphemes is uncertain and there are alternative interpretations of the structure of the verb constructions that include a 'classifier' morpheme, with some researchers considering the movement as the root and the handshape as a proform or agreement marker affix (e.g. Supalla, 1982); others favouring the handshape as a central element (Engberg-Pedersen, 1993); and yet others arguing that the terms 'root' and 'affix' cannot be applied to simultaneous constructions of this kind where none of the elements are more 'basic' than others (Johnston \& Schembri, 1999). Schembri (2003) and Zwitserlood (2012) provide a comprehensive outline of the arguments for and against particular interpretations of these constructions, but these debates will not be covered in this thesis. The term 'polycomponential sign' advocated by Schembri has been adopted here as a neutral term that avoids committing to a particular morphemic analysis.

Polycomponential signs are present in most signed discourse but are especially prominent in narratives (e.g. Morford \& MacFarlane, 2003 for ASL). Signers may construct a unique polycomponential sign as a one-off description, creating what Brennan (1992, p. 46) referred to as a 'DIY' sign rather than an 'off the shelf' lexical item. These forms can be understood by interlocutors because of the conventional meaning of the individual subcomponents. However, Johnston and Schembri (1999) argued that the meaning of these one-off constructions is only specified within the context and is not more than the meaning contributed by each of the components. In other words, these constructions are not lexemes. 
The significance of polycomponential signs to lexicography lies in the fact that they are extremely productive (Brennan, 1990; 1992). A large proportion of the core lexicon of signs has its origins in a polycomponential construction that acquired a more specific, contextindependent meaning over time. An example given by McKee and Vale (forthcoming) is the sign lexicalised as BUNGY-JUMP in NZSL (see figure 2.5), where the dominant hand represents the legs, the non-dominant hand represents a flat surface, and the movement shows a jump off the surface. This lexicalised form has a more specific meaning than just jumping off a platform. Individual variations in the movement path or the distance between the fingers in this lexeme are no longer meaningful: a citation form of the sign can be said to exist. The meaning of the individual components, however, not only remains available for use in other polycomponential constructions, but can also be 're-activated' in a lexical sign (Brennan, 1990) or be 'delexicalised' (Johnston and Schembri, 1999). For example, a signer could make reference to a particular location on the platform by pointing at the flat hand; substitute a curved hand to indicate jumping from a bridge; vary the handshape to show the position of the legs; or change the movement path to show the speed of the jump more precisely. The boundaries between core lexemes and non-core polycomponential signs therefore remain unclear.

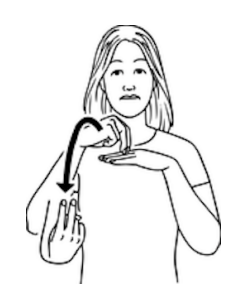

Figure 2.5: A lexicalised polycomponential sign—BUNGY-JUMP

\subsubsection{Indexicals or pointing signs}

Pointing signs (often referred to as 'indexicals' in the sign language literature) are prevalent in all described sign languages. These signs are used to refer to directions, locations, people and objects. Cormier, Schembri, and Woll (2013) discussed the features that sign language indexicals have in common with pointing gestures co-occurring with speech in spoken languages. Sign languages often use the same handshape for pointing as gestures in speakers of the surrounding spoken language. In both signed and spoken languages, pointing can be used with concrete or abstract referents, in the real environment or an imagined space, and to actual or metaphorical locations. In both types of languages, the handshape of the pointing gesture may change according to the context, and reference can be either deictic or anaphoric. In sign languages, there are also more complex indexicals which include the 
incorporation of number (e.g. 'three of us'), possession or reflexiveness through changes in the handshape (e.g. Johnston \& Schembri, 1999).

Pointing signs (treated as lexicalised signs in these studies) are amongst the most frequently used signs in the few frequency studies that have been carried out. Morford and MacFarlane (2003) listed a 'non-first-person indexical' and the first person form 'I, me' as the two most frequent signs in their study on ASL, and their top ten results also included first person and non-first person possessives. McKee and Kennedy (2006) found similar results for NZSL, with four 'pronominal' and 'locative' indexicals and a first person possessive in the ten most frequently used signs. Johnston (2012) compared these two studies with data from a large annotated corpus of Auslan and again found similar results.

Discussions on the role of pointing signs have centred around the question whether different types of pointing can be distinguished, and in particular whether a subset of pointing signs constitutes a pronominal system in sign languages. Johnston and Schembri (1999) argued that pointing signs should be classed together as 'deictics', whether they functioned as 'pronouns' (you, us, I, he), 'demonstratives' (this, that), 'locative adverbials' (here, there) or references to 'parts of the body' (e.g. a point to the nose, elbow, or knee). Cormier et al. (2013) also noted a determiner function, previously identified by Liddell (1980) and Lucas and Valli (1990) amongst others, where a pointing sign is part of a noun phrase and establishes a particular location as a reference point for further discourse. In all of these cases, determining whether the sign points to a referent or to the place in which that referent is located relies on the discourse context rather than on differences in form. Cormier et al. (2013) argued that these signs share features with spoken language pronouns as well as with co-speech pointing gestures, and therefore proposed that pronominal signs may be "grammaticalised forms of locative pointing gestures". Further evidence for this analysis comes from the fact that these signs are used much more frequently in sign languages than they are as co-speech gestures.

In lexicalised and grammaticalised form, arguments have been made for a pronominal system with first, second and third person forms (e.g. Friedman, 1975; Klima \& Bellugi, 1979). However, other researchers have maintained that the only distinction that can be made is between first person and non-first person forms (e.g. Engberg-Pedersen, 1993; Emmorey, 2002; Liddell, 2003). In the first person form, the location is the body of the signer (the centre of the chest in most documented cases), but in all other forms the direction of the pointing sign depends on the location of the referent in the (real or signing) space. There is therefore 
no 'listable' location parameter (Rathmann \& Mathur, 2002) since any of the locations within the signing space are potentially meaningful, and a citation form cannot be said to exist (Johnston \& Schembri, 1999). Johnston and Schembri (2007) stated that, although pointing signs are therefore not strictly lexemes, they considered the pronominal and possessive pointing signs to be "incompletely specified lexicalised signs" belonging to the core native lexicon. Similarly, signs indicating body parts by pointing are not considered to be true lexemes, in contrast to pointing signs that have acquired a more specific meaning such as a point to the ear in Auslan meaning 'to hear' (Johnston \& Schembri, 1999).

\subsubsection{The non-native lexicon}

\subsubsection{Fingerspelling}

Manual alphabets were historically in use in various hearing communities, including as a communication method for certain religious orders. Around the 16th century these alphabets were introduced into the education of deaf children as tools for speech training or to represent written language (Padden \& Gunsauls, 2003).

In manual alphabets of Western languages, each letter of the (Roman) alphabet is represented by a hand gesture and these gestures are combined sequentially to 'spell out' words of the spoken language. Variants of a common one-handed alphabet are in use in much of Western Europe and in the United States. In the United Kingdom, Australia and New Zealand, a two-handed alphabet is used. The practice of spelling out words is commonly referred to as 'fingerspelling'.

For sign language users, fingerspelling fulfils a range of functions. In many cases, fingerspelling may be interpreted as a form of codeswitching by bilingual Deaf people. Many of the 11 functions of fingerspelling listed by Sutton-Spence (as cited in Brennan, 2001) are of this type: for example, fingerspelled words are used to introduce concepts for which no sign (yet) exists, or in situations where one of the interlocutors is not familiar with a particular sign; idioms or acronyms from the spoken language may also be fingerspelled, and it may be used as a form of euphemism to avoid using explicit signs. In addition, Brennan (2001) mentioned the use of fingerspelling as a clarification strategy, especially in technical discourse where signs may not yet have been established.

In some sign languages, fingerspelling is used very frequently. Brentari and Padden (2001) reported that in ASL, between 7-10\% of vocabulary in everyday discourse consisted of fingerspelled words, and Padden and Gunsauls (2003) gave an even higher estimate of 12- 
$35 \%$. Because of this frequent use, fingerspelling was found to be a productive part of the ASL lexicon. Battison (1978) first discussed how fingerspelled forms were adopted into ASL as loan signs, undergoing modifications to conform to the formation rules of native signs. For example, the movement patterns might be reduced by shortening the fingerspelled word to only a few letters, and handshapes of individual letters might be assimilated to blend with preceding or subsequent letters. The resulting forms are fully lexicalised and some are able to undergo changes in location or movement to express spatial arrangements of referents, in a similar way to signs from the native lexicon. Brentari and Padden (2001) further elaborated on lexicalisation mechanisms of fingerspelling, including:

- $\quad$ spelling a complete word;

- $\quad$ spelling an abbreviated version of the word;

- $\quad$ using the first letter of the word;

- incorporating the handshape of an individual letter into an existing verb or as part of a polycomponential sign.

Both Brentari and Padden and Brennan (2001) found that lexicalised fingerspelling forms ranged from the full loan signs described by Battison (1978) to routinised, fluidly fingerspelled words that function as stable lexemes but are deliberately kept non-native. According to Brennan, “...the intention here is to enable the English-ness of the form to remain apparent." (p. 63).

In New Zealand, the two-handed fingerspelling alphabet was not introduced into deaf education until 1979. As a result, fingerspelling does not fulfill the same productive role in NZSL that has been noted for ASL, BSL, or Auslan. Pivac Alexander (2008) found that fingerspelling in NZSL occurred much less frequently $3.2 \%$ of the data, including some forms based on the one-handed ASL alphabet). Usage of fingerspelling was affected by sociolinguistic factors, with younger, female, northern and non-Māori signers more likely to use fingerspelling. Older generations did not use any fingerspelling at all, instead using other strategies such as mouthing. Lexicalised fingerspelling in NZSL was found to predominantly consist of single-letter forms (for example, a fingerspelled $\mathrm{M}$ for 'mother') or abbreviations (e.g. T-V or I-D) with fewer complete words. Pivac Alexander also found that fingerspelling is more restricted as to word category than has been reported for other sign languages: in NZSL, it is mainly used for proper nouns, common nouns, a few adjectives and affixes, and not at all for other word classes such as verbs, adverbs or conjunctions. 


\subsubsection{Mouth gestures and mouthing}

While Stokoe's original research (1960) mainly focused on the manual component of signs, it was soon found that movements of the face and body were also significant. In particular, mouth movements were found to accompany much of signed discourse. Two distinct types of mouth patterns have been identified in many sign languages: mouth gestures and mouthings. (Boyes Braem \& Sutton-Spence, 2001).

Mouth gestures are mouth patterns unrelated to the spoken language, although Johnston, van Roekel, and Schembri (2015) suggest that further research is required to investigate possible commonalities between mouth gestures used by Deaf signers and by people from the surrounding spoken language community. Liddell (1980) identified a number of mouth gestures in ASL that fulfilled adverbial or adjectival information, such as manner and degree, definiteness, or intensity. Similar types of mouth gestures have been found for many sign languages, including many of the European sign languages described in Boyes Braem and Sutton-Spence (2001) (see also Crasborn, van der Kooij, Waters, Woll, \& Mesch, 2008), in Auslan (Johnston \& Schembri, 2007) and in NZSL (McKee, 2007). In addition to their adverbial role, mouth gestures may enact actual mouth movements, for example a chewing motion accompanying a manual sign CHEW or puffed out cheeks with a sign BALLOON. A third category of mouth gestures is identified by Woll (2001) as 'echo phonology', since the mouth movements are coordinated with the actions of the hand: for example, the open mouth closing in parallel with a closing hand movement. These mouth gestures have no fixed meaning but are nevertheless an obligatory part of a sign, for example the 'shhh' mouth gesture that is an obligatory part of the sign THERE-EXISTS in Sign Language of the Netherlands (Bank, Crasborn, \& van Hout, 2011). In combination with other non-manual features, mouth gestures may also convey emotions (for example, opening the mouth wide to signify surprise) or signal negation and other grammatical processes.

The status of mouth gestures varies: semantically empty mouth gestures are considered purely phonological and a part of the lexical signs they accompany; adverbial mouth gestures may be considered to be bound morphemes since they are not tied to a particular lexical item and have an independent meaning; and enactment mouth gestures are similarly not associated with an individual lexical item since they can convey different information than the manual sign they occur with (Crasborn et al., 2008). Regardless of their status, mouth gestures are part of the native lexicon. 
By contrast, mouthings are a non-native influence, since they are derived from spoken words. Some mouthings are recognisable as complete (unvoiced) pronunciations of the spoken words, whereas other mouthings are reduced in form. Rates of mouthing vary in different sign languages: they are especially common in many European sign languages (Boyes Braem \& Sutton-Spence, 2001) but were initially reported to be much less frequent in ASL. However, Nadolske and Rosenstock (2007) found mouthing to be significant in ASL as well. It is possible that mouthing has been given less attention because fingerspelling is very productive in ASL and fulfils some of the same functions (Brentari \& Padden, 2001). McKee (2007) reported that on average, 73\% of signs in NZSL were accompanied by mouthings; Boyes Braem (2001) reported even higher rates of $76-80 \%$ in Swiss German Sign Language.

Rates of mouthing have been reported to vary according to genre, with fewer occurrences in narratives than in informative texts (Sutton-Spence \& Day, 2001). However, McKee (2007) found no genre differences in NZSL between conversational and interview data. Many studies have identified that mouthings occur most frequently with nouns (e.g. Boyes Braem \& Sutton-Spence, 2001; McKee, 2007). Boyes Braem (2001) found that mouthings occurred with open class elements rather than closed class elements.

Sociolinguistic factors have been found to influence the rate and type of mouthings. Boyes Braem (2001) compared early and late deaf learners of Swiss German Sign Language and found similar proportions of mouthing, but a qualitative difference in the kind of mouthing, with late learners producing more complete mouthings and fewer reduced forms. McKee (2007) identified age as a variable with older signers in NZSL producing the highest ratio of mouthing. Changes in the education of deaf children in New Zealand underlie this age variation: older signers were exclusively taught with strict oralist methods, whereas NZSL started to be used in education in the mid-1980s, and attitudes towards mouthing changed accordingly.

Mouthing generally fulfils semantic rather than grammatical functions. The main functions of mouthing identified by Schermer (2001) are to disambiguate between signs that have the same manual form, or to specify or complement the information provided by the manual sign. In addition to these lexical functions, Boyes Braem (2001) also included a discourse function where mouthings establish linguistic reference (for example, by clarifying names or abbreviations) and prosodic functions where mouthing adds emphasis or stress, or binds a phrase by stretching a mouthing across several signs (see also Schermer, 2001; McKee, 2007; 
Crasborn et al., 2008; Bank et al., 2011). Boyes Braem also identified the productive role of mouthing as a mechanism to extend the meaning of existing manual signs (see also McKee, 2007). This is a common way of deriving specific technical meanings from generic signs. An example would be the mouthing of 'precipitation' accompanying the existing sign RAIN.

Debates around the status of mouthing are ongoing. Some authors (e.g. Ebbinghaus \& Hessman, 2001) maintain that mouthing is an independent communication mechanism that should not be seen as an integral part of sign languages. Others have argued that mouthing is a particular kind of code-mixing whereby signs and spoken words are simultaneously produced and 'blended' together. The term 'code-blending', coined by Emmorey, Borinstein, and Thompson (2005), has been adopted to describe this phenomenon. Arguments for a code blending interpretation include the fact that mouthing is semantically redundant in some cases; that some mouthings occur as free-standing morphemes, without a manual sign; and that there can be great variation in the mouthings produced with a particular sign, so that different spoken words or different reductions of the same word are produced on different occasions (Schermer, 2001). As previously mentioned, mouthings can also stretch across preceding and following signs, suggesting that mouthings are not bound to individual signs. On the other hand, there is evidence to support an interpretation of mouthing as borrowing. Mouthings are an obligatory part of some signs, and Deaf signers judge these signs as not well-formed when they are produced without the mouthing (Schermer, 2001). Boyes Braem (2001) reported that highly frequent signs were accompanied by stable mouthings, and that both one-off borrowings and more established borrowings were common. Some mouth patterns are fully lexicalised and, in their reduced form, may no longer be recognised as deriving from a spoken word. Schermer (2001) found differences between the mouthings that accompanied signs in isolation and those that were produced with the same signs in context. Recent corpus-based studies confirmed variability in context; for example, Bank et al. (2011) found that signs that were typically accompanied by a mouth gesture in citation form could be accompanied by either a mouth gesture or a mouthing in context.

The uncertainty around the status of mouthings is one of the factors that have a significant impact on the representation of lexemes in sign language dictionaries, as will be further discussed in the chapter's conclusion. 


\subsubsection{Loan translations}

Loan translations, whereby spoken language words or phrases are transferred literally into the sign language, are another non-native sign formation process. Loan translations may be oneoff occurrences of code-switching by bilingual Deaf people, either unconsciously or with a specific intent to mark what is being said as originating from the spoken language (SuttonSpence \& Woll, 1999). Loan translations may also be a form of language play where puns on the spoken language word are intended (Sutton-Spence, 1999).

Loan translations are highly productive in many sign languages, especially in certain domains. Loan translations of compounds are common: for example, GREEN-HOUSE, BOYFRIEND and WORK-SHOP (see figure 2.6) and may not be perceived as borrowings at all (Leeson \& Saeed, 2012; Johnston \& Schembri, 2007).
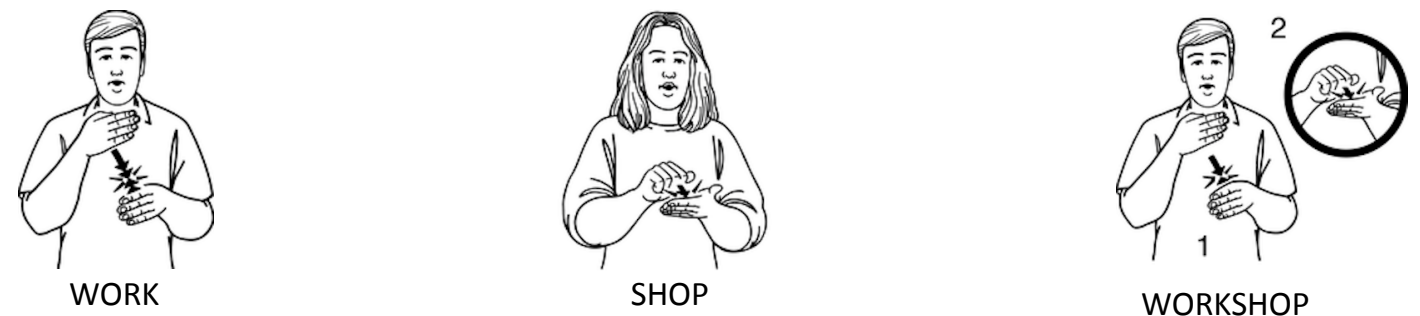

Figure 2.6: A loan translation compound in NZSL-WORKSHOP

Sutton-Spence and Woll (1999) mentioned English idioms as another common area for loan translations into BSL. Sutton-Spence (1999) and Johnston and Schembri (2007) both stated that place names and proper names are another common area for borrowings via loan translation. In NZSL, the place name Greymouth is signed using a combination of the signs GREY and MOUTH. The place name Paraparaumu in NZSL is rendered by the sign PRAM, based on a common shortened pronunciation of the place name in New Zealand English.

Some loan translations become lexicalised over time, although particular signs may not become widely accepted, especially where established native signs already exist or where Deaf people reject the adoption of spoken language grammatical and semantic patterns, preferring to call on native sign formation processes instead (Johnston \& Schembri, 2007).

Loan translation processes interact with native sign formation processes, so that initial borrowings may undergo morphological changes to become more native-like. McKee \& McKee (2013) gave an example of an NZSL sign 'open-minded' where the original form consisted of the two separate signs OPEN and MIND, but a current variant has relocated the two-handed sign OPEN to the forehead location of MIND. 


\subsubsection{Borrowing from other sign languages}

In addition to contact with surrounding spoken languages, sign languages are also increasingly in contact with each other. Johnston and Schembri (2007) mentioned greater opportunities for travel as one of the main reasons and this is echoed by McKee and McKee (2011), who stated that travel and immigration are both factors in unplanned borrowing of foreign signs into NZSL, especially from Auslan, ASL and BSL. More recently, an anecdotal source of borrowing is increased exposure to other sign languages, in particular dominant sign languages such as ASL, through signed videos shared via social media and the internet.

Lexical borrowing is often motivated by a need to expand the vocabulary, for example when Deaf people enter new areas of education and employment. Sign languages with a longer documented history of use and larger Deaf communities, such as ASL and BSL, may be seen as prestigious by smaller communities such as the New Zealand Deaf community. These larger sign languages are also likely to have an established vocabulary in specialist areas and this vocabulary may spread not only through personal contact, but also through available (print and video) resources (Johnston \& Schembri, 2007). Domains such as religion and education have therefore seen frequent borrowing into both Auslan and NZSL. Another frequent domain for borrowing between sign languages is place and country names, where signs established by Deaf people from the country or place in question may replace local signs (Matthews, McKee, \& McKee, 2009).

Borrowing from other sign languages also extends to the one-handed fingerspelling alphabet. Brennan (2001) described forms based on the Irish one-handed fingerspelling alphabet entering BSL; Johnston and Schembri (2007) noticed a similar influence through the establishment of Irish-staffed Catholic schools in Australia in the late $19^{\text {th }}$ century. The strong influence of ASL on other sign languages is also apparent here. Pivac Alexander (2008) found that $8.3 \%$ of fingerspelling tokens in her NZSL data were based on the one-handed ASL alphabet, either as single letter forms (for example, 'L' for the place name 'Levin' or 'O' for the month 'October'), or as complete borrowed lexical forms such as the ASL sign O-K or the initialised sign LANGUAGE.

\subsection{Summary and discussion}

In summary, natural sign languages have evolved in, and are mainly used by minority Deaf communities surrounded by a wider hearing population. Sign languages have until recently only been used in limited domains and experience a great deal of variation, arising partly 
from the lack of a written form. Due to continued contact between spoken and sign languages, many Deaf individuals are to some extent bilingual and may show spoken language influences in their signing. Since the majority of deaf people are born to hearing parents, there are also a large number of individuals who do not acquire a sign language until a later age. In spite of some recent recognition of their linguistic status, many sign languages are now considered endangered with diminishing numbers of users due to disrupted transmission patterns, fewer traditional sites of transmission, and limited institutional support.

The lexicon of sign languages was found to have a relatively small core of established, completely specified lexemes with a context-independent meaning. The larger part of signed discourse was found to consist of non-core signs such as polycomponential (depicting) signs and pointing signs, and non-native influences such as fingerspelling, mouthing, loan translations, and borrowed signs.

The distinction between signs in different parts of the lexicon is often unclear, since many core lexemes will have originally been derived from productive non-core and non-native elements. The lexicon in sign languages should therefore be seen as a continuum, containing partly lexicalised signs as well as completely specified lexemes (Johnston \& Schembri, 2007). For sign language lexicographers, it can be difficult to determine the lexical status of some signs and to decide whether these signs should feature as lemmata in the dictionary. Johnston and Schembri (1999) argued that sign language dictionaries should restrict themselves to documenting the core lexicon, since the non-core and non-native elements are better dealt with in a grammar. However, given the scarcity of accessible grammars and learning resources for many sign languages (for example, a learner-orientated reference grammar of NZSL (McKee, 2015) has only recently become available) and the uncertain lexical status of many signs, it has not always been possible to take this strict approach. Moreover, Brien and Turner's (1994) viewpoint was that sign language dictionaries have a role in documenting the productive lexicon alongside the core lexicon, as this part of the lexicon contains the very richest and most culturally bound parts of these languages. The lengthy introduction to the Dictionary of British Sign Language/English by Brennan (1992) provided extensive information on the productive lexicon of British Sign Language. In addition, Brien and Turner advocated the use of 'proto-definitions' in the dictionary entry itself, highlighting the roots of signs such as CATERPILLAR in productive elements showing an insect crawling up a narrow thin object. The importance to learners of certain productive or inflected forms as 
equivalents for frequently used spoken vocabulary can also warrant the inclusion of these signs in a bilingual sign language dictionary (McKee \& McKee, 2013).

Even when a decision has been made to enter a sign into the dictionary, the citation form can be difficult to determine. Due to the lack of a written standard form, signs vary a good deal in individual production (Zwitserlood, Kristoffersen, \& Troelsgård, 2013). Zwitserlood et al. also mentioned difficulties in determining the citation form for signs that are routinely modified: for example polycomponential signs such as GIVE (figure 2.7) where the handshape classifies the type of object given, or signs such as X-YEARS-OLD (figure 2.8) that incorporate different number handshapes to indicate age.
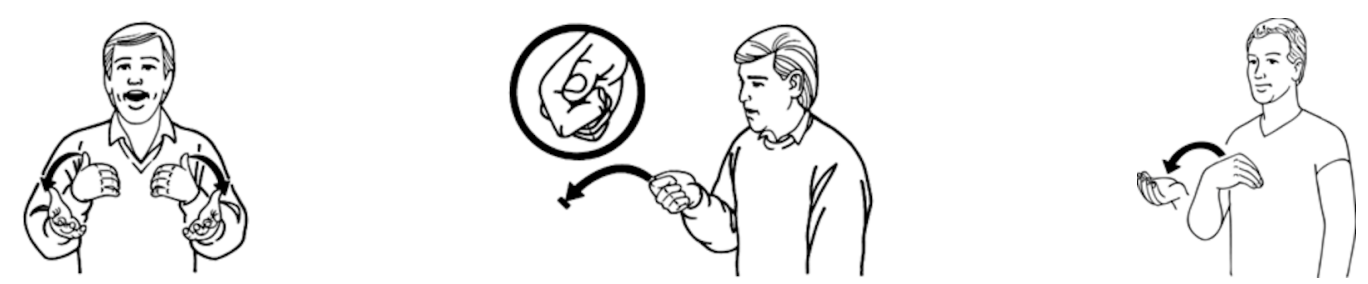

Figure 2.7: Three forms of a polycomponential NZSL sign—GIVE

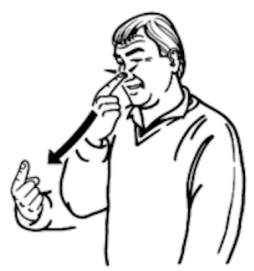

Figure 2.8: An NZSL sign showing number incorporation-ONE-YEAR-OLD

Further issues affecting lemmatisation and lemma description in sign language dictionaries are:

- the high level of polysemy in sign languages (Zwitserlood et al., 2013; McKee \& McKee, 2013);

- $\quad$ distinguishing between variant forms and synonyms (Zwitserlood et al., 2013);

- uncertainty around the assignment of word classes, with many signs showing no significant differences in form between noun and verb (McKee \& McKee, 2013);

- the debated status of non-native elements such as mouthing (McKee \& McKee, 2013 (see also section 2.2.5.2 - Mouth gestures and mouthing);

- the inclusion of certain frequently used inflected forms as separate lemmas (McKee \& McKee, 2013). 


\subsection{Conclusion}

This chapter has reviewed relevant literature on the sociolinguistic context and the linguistic description of the lexicon of sign languages. The sociolinguistic context has both direct and indirect implications for the current thesis. Firstly, as was described in Chapter 1 Introduction, the thesis as a whole is motivated by a desire to contribute to the promotion and maintenance of sign languages through practical lexicography. The aims of sign language lexicography will be discussed in further detail in Chapter 3 - Sign language lexicography. Secondly, it will be argued that signed folk definitions can give prominence to Deaf people's language use and cultural knowledge since they "prioritise the language under study over a language of analysis" (Dingemanse, 2015, p. 216). This prioritisation of Deaf people's perspectives is especially crucial given the pervasive influences of the dominant spoken languages that have been outlined here. Thirdly, dictionary users have become a central focus for lexicography in recent years. The users of sign language dictionaries are extremely varied (see section 3.2.2 - Envisaged user groups) and potentially include members of the hearing majority, Deaf native users and deaf sign language learners. To understand the user needs of these various groups, the sociolinguistic context has to be borne in mind. Fourthly, there are implications for the recruitment of research participants for the studies in this thesis. Folk definitions are paraphrases by native speakers of a language; however, given the nature of Deaf communities, true native sign language users who have acquired the language from birth are scarce. Finally, the frequent language contact between spoken and signed languages and the largely bilingual status of the Deaf community will impact on the collection of signed folk definitions, since participants may typically rely on spoken or written explanation strategies in their everyday life, rather than having signed "natural explanatory routines" (Dingemanse, 2015). These last two issues will be addressed further in Chapter 6 Folk definitions.

The nature of the sign language lexicon as described in this chapter implies that sign language dictionaries are likely to contain lemmas of various types, including marginal lexemes and lexemes that may have originated from the non-core and non-native lexicon. For the current thesis, a cross-section of lexemes of different types will therefore be selected to function as stimuli for eliciting folk definitions.

There are many areas in which the linguistic description of the lexicon is not yet advanced enough to provide guidance to lexicographers. Wilcox (2003), in his evaluation of an early effort to create a CD-ROM dictionary of ASL, pointed in particular to the lack of attention to 
semantics in sign linguistics. According to Lew (2007), there is a disjunction between formal linguistic semantics, which investigates how words mean, and the semantics that is the focus of lexicography, focusing on what words mean. Nevertheless, the gaps in our knowledge about how signs convey meaning are likely to affect the description of sign lemmas in dictionaries. The proposed study of signed folk definitions, as well as having a practical application in sign language dictionaries, may also contribute to our knowledge of sign language semantics by investigating the form that such explanations take.

In the next chapter I will outline further factors that have a bearing on the lack of definitions to date by examining the history and purposes of sign language lexicography and the format and types of sign language dictionaries. 


\section{Chapter 3: Sign language lexicography}

\section{Introduction}

This chapter examines the history of practical and theoretical sign language lexicography. The format of sign language dictionaries has been shaped by the fact that there have been limited resources for dictionary making, so that a single dictionary has often had to fulfill multiple purposes and meet the needs of different envisaged user groups. A major limiting factor has been the lack of an orthography for sign languages, which has implications for the way in which sign lemmas and associated information are represented in dictionaries. A number of typological distinctions will be applied to sign language dictionaries to show that there is a smaller range of formats compared to dictionaries of spoken languages. In particular, sign language dictionaries have to date been almost exclusively bilingual.

The chapter concludes by examining the potential offered by electronic lexicography. Corpora, increasingly central in general lexicography, are still in the very early stages of development for sign languages. Nevertheless, corpus evidence is informing several aspects of modern online sign language dictionaries. Other aspects of electronic lexicography may enable online dictionaries to address the needs of multiple users simultaneously. These developments make it possible to consider the inclusion of signed definitions.

\subsection{History and purposes of sign language dictionaries}

Due to the close contact between Deaf people and the surrounding hearing majority, there have always been hearing individuals who wanted to communicate with Deaf people. This communication need has historically been one of the major drivers for creating sign language dictionaries. Many such dictionaries were therefore created by educators, members of religious orders, or welfare workers (Zwitserlood, 2010). Early sign language 'dictionaries' were largely simple lists of words from the spoken language, accompanied by an image or description of a sign. An early example dates from late $18^{\text {th }}$ century France (Zwitserlood, Kristoffersen, \& Troelsgård, 2013), but Brien and Turner (1994) found that sign language dictionaries produced until the early 1990s still almost exclusively followed this same simple glossary format and focused on the communication needs of hearing users in their selection of concepts for inclusion in the dictionary. 
The influential research by Stokoe (1960) on the linguistic properties of American Sign Language also brought about another motivation for creating a sign language dictionary. Stokoe worked in the anthropological tradition, in which field studies frequently involved documenting the lexicon and grammar of the community being investigated. The Dictionary of American Sign Language on Linguistic Principles (DASL) (Stokoe et al., 1965) took a descriptive linguistic approach and was the first dictionary in which a sign language was the source language.

The number of sign language dictionaries being produced increased sharply after the publication of the DASL. A stocktake of sign language dictionaries worldwide, initiated by the World Federation of the Deaf (Carmel, 1992) revealed that the number of publications doubled between 1960-1970 and again between 1970-1980. In the early 1990s alone, around 120 sign language dictionaries were published worldwide. However, few of these resources were based on the linguistic principles set out by the DASL. Brien and Turner (1994) noted that educational policies in the UK in the time leading up to the production of the Dictionary of British Sign Language/English (Brien, 1992) still emphasised the use of signs as a support to spoken English and did not acknowledge sign languages as natural languages independent from the spoken language. The makers of sign dictionaries for use in education therefore paid little attention to the way in which signs were used in the Deaf community and often coined signs themselves to fill perceived gaps (Johnston, 2003).

As linguistic research on sign languages increased, a number of descriptive general dictionaries appeared over a short time in the 1990 (e.g. Johnston, 1989 a; Suwanarat et al., 1990; Brien, 1992; Kennedy et al., 1997). These dictionaries returned to the purpose of documenting the existing lexicon in use by the Deaf community, with the wider aims of contributing to sign linguistic research, enhancing the status of the sign language being documented, and promoting its acceptance by the majority community.

The importance of dictionaries to the Deaf community's status and linguistic rights is recognised by the World Federation of the Deaf (Haualand \& Allen, 2009). Sign language dictionaries may also contribute to achieving legal recognition or protection for the sign language in question (p. 23). The status-enhancing effect of dictionaries, however, is often tied to the part dictionaries play in codifying the language, leading to the development of a standard (Zgusta, 1971). Standardisation may happen inadvertently, for example when a particular variant is more strongly represented amongst the informants consulted during 
data collection (e.g. Schmaling, 2012); but it has often also been an explicit aim of sign language dictionaries. A standard sign language is seen as desirable since it would facilitate the use of the sign language for educational purposes (Stokoe et al., 1965) and the creation of shared resources and media. In some cases, government support for sign language-related projects may be dependent on a standardisation project, as was the case with Sign Language of the Netherlands (Schermer \& Koolhof, 2010). For these reasons, standardisation has also been supported and encouraged by Deaf communities themselves, although not without controversy (Johnston, 2003). The goal of standardisation may be in conflict with the professed descriptive aims of sign language dictionaries. However, Lucas (2003) argued that both the documentation of variation and the unification of a sign language through a standard form are "central to the continuing empowerment of Deaf people all over the world." (p. 339). Sign language lexicographers cannot avoid making decisions on how to deal with variation in the dictionaries they produce, and they are urged by Lucas to define their perspective clearly.

The acceptance of sign languages in education led to the increased participation of Deaf sign language users in new areas of education and employment, and a resulting need for signed technical vocabulary. Sign language dictionaries started to be developed that covered a particular domain or curriculum subject: for example, health, science, or religious signs. These specialist dictionaries included Deaf people as a target user group, alongside hearing educators and service providers such as social workers, or later sign language interpreters. While new signs were originally often coined by hearing educators, in more recent years it has been stressed that native Deaf signers should be involved in the process of developing new vocabulary. A team approach has become common to ensure a combination of native language knowledge, subject-specific content knowledge, and linguistic expertise (Schermer, 2006).

As sign languages have become more visible in the wider society, hearing people with no previous connection to the Deaf community have become interested in learning to sign and the demand for sign language dictionaries and other learning resources has increased. With the development of sign language interpreting as a profession, advanced hearing sign language users have also become a target user group of sign language dictionaries, both during their training and as professionals who come into contact with a wide range of social and situational sign language variants. 
While for many sign languages, the first documentation projects are still in progress, sign languages in countries with larger Deaf communities may now have several 'generations' of sign language dictionaries, allowing for diachronic comparisons. The present endangered status of some sign languages has made the preservation of signs used by older Deaf community members another goal for dictionary makers. Just as in other endangered language communities (Ogilvie, 2011), sign language dictionary makers are motivated to contribute not only to language maintenance, but also to language revitalisation.

\subsection{The format of sign language dictionaries}

Before examining whether distinct types of sign language dictionaries can be identified, there are some general observations that can be made about their format in comparison with spoken language dictionaries.

Firstly, sign language dictionaries have generally been smaller works, typically containing around 2,000-5,000 sign lemmas (Zwitserlood, 2010). The difference in size is partly due to the nature of the sign language lexicon, which was found to have a small core of established lexemes. In addition, sign languages were until recently only used in limited domains, leading to a more restricted vocabulary in the past. A further reason is that sign languages are 'lesser-resourced' languages (Prinsloo, 2012). Dictionaries of lesser-resourced languages, according to Prinsloo, typically are smaller, treat a smaller number of headwords, and provided limited data types, because financial and human resources for dictionary making are scarce.

Secondly, although the type and amount of information provided in the microstructure of dictionaries varies according to the kind of dictionary, sign language dictionaries generally provide less information than their spoken language counterparts. As tools for language documentation, dictionaries aim to provide extensive information not only on the form and semantics of lemmas, but also on grammar, variation, etymology et cetera. The fact that sign linguistics has only recently emerged as a discipline means that sign language dictionary makers have had to deal with incomplete and at times contradictory linguistic models when deciding how to categorise and describe sign lemmas. Gathering detailed, reliable evidence of sign language use is also problematic given the limited number of 'native' sign language users and the small number of available texts. 
There are three further factors that have shaped, and continue to shape, the format of sign language dictionaries.

\subsubsection{Representing signs without a written form}

A substantial influence on the format of sign language dictionaries has been the fact that sign languages are unwritten languages. A common step in field lexicography of unwritten minority languages is the creation of an orthography, which raises decisions about adapting existing written systems from another language or group of languages, or creating a custom set of writing symbols and conventions (Frawley, Hill, \& Munro, 2002). Stokoe (1960) devised a sign notation system that used collections of symbols showing the location, handshape, and movement of signs. This system was subsequently used to represent sign lemmas in the Dictionary of American Sign Language on Linguistic Principles (DASL). An adaptation of the Stokoe Notation System was used in the Dictionary of British Sign Language/English (Brien, 1992). Further notation systems have been developed and used in sign language dictionaries, most notably HamNoSys (Prillwitz, Leven, Zienert, Hanke, \& Henning, 1989) and SignWriting (Sutton, 2002). However, none of these systems have been successfully established as common writing systems. Stokoe Notation and HamNoSys were not intended to function as systems for writing down larger texts, but rather as analytical tools for the transcription of individual signs. Although SignWriting has been used to create larger written texts, its use by Deaf people in everyday life has not become widespread.

In sign language dictionaries, the use of notation systems has been restricted to representing the sign lemma itself, and sometimes acting as an alternative organising principle (see section 3.4.6 - Macrostructure). Notation systems have not been used as a dictionary metalanguage; in other words, apart from the sign lemma, all other information in the entry and in front and back matter has been provided in the written form of one or more spoken languages.

Although entries in the DASL only contained Stokoe notation and written English descriptions, more usually sign lemmas have been represented through images. Until the advent of electronic dictionaries this was the only method that was accessible to a nonspecialist dictionary user. Photographs or line drawings have been used, either annotated with arrows or symbols or presented as a sequence to show the movement of signs. Because as static representations of a three-dimensional moving sign, images are not always transparent, they have sometimes been accompanied by a written description or 'recipe' on how to 
produce the sign. The main shortcoming of using images is that they take up a good deal of space. This places a further restriction on the number of entries a print sign language dictionary can contain, and again prevents the use of the sign language as a metalanguage in the dictionary (Zwitserlood, 2010).

In the absence of a custom method to represent longer signed text, the practice of glossing continues to be common in sign language research and has also been applied in sign language dictionaries. Glosses are words or phrases from the written / spoken language that approximate the meaning of the sign they represent. Although glosses have also been used in spoken language dictionaries, in particular acting as translational equivalents in bilingual dictionaries, the shortcomings of glosses as semantic equivalents have long been noted (e.g. Zgusta, 1971; 1987). The issue of inequivalence between source language lexical items and target language glosses will be discussed in detail in section 5.2 - Equivalents or glosses.

The use of glosses in sign language dictionaries is particularly problematic. In the case of spoken languages, Pizzuto and Pietrandrea (2001) argue, glosses act as a supplement independent from the written representation of the source language lemma. In the case of sign languages, however, the gloss is the only written representation of the sign and is therefore equated with the sign itself. As a consequence, semantic and grammatical properties of the gloss may be misinterpreted as properties of the sign (Vermeerbergen, 2006). Johnston $(2008,2010)$ urges sign language lexicographers and corpus linguists to assign unique identifiers (so-called 'ID glosses') to each sign in order to avoid such misinterpretations. However, Kristoffersen and Troelsgård (2012) point out that using one fixed gloss in a dictionary entry, database or corpus, while having the advantage that it identifies a sign unambiguously, fails to alert the dictionary user that other meanings are possible in different contexts.

In electronic dictionaries, the use of video recordings, either of real sign language users or of animated avatars, has resolved some of the difficulties of representing signs. Video recordings more accurately reflect the form of signs and they can also be used to show longer signed texts, such as example sentences or, in principle, definitions. However, Kristoffersen and Troelsgård (2012) discuss the shortcomings of videos in that video recordings by themselves cannot be searched, cross-referenced or ordered in a database, or displayed easily in search results. It is therefore likely that other representations such as static images, notations, and glosses will continue to be a part of the dictionary entry in electronic dictionaries. 


\subsubsection{Envisaged user groups}

One of the foundations of modern lexicography is that different types of dictionary suit different purposes and different users. It is therefore important to consider the user groups that sign language dictionary makers have targeted as a further influence on the format of sign language dictionaries.

From the brief history of sign language lexicography in section 3.1 - History and purposes of sign language dictionaries, it can be seen that hearing people with personal and professional connections to the Deaf community have been the first and largest targeted user group of sign language dictionaries. The forewords to many sign language dictionaries reveal a large number of additional envisaged user groups, ranging from specific professionals such as teachers and interpreters, to Deaf adult sign language users, deaf children, and other people who use signs to support speech. Sign language researchers are also potential dictionary users (e.g. Kristoffersen \& Troelsgård, 2012).

While in the case of the simpler unidirectional glossaries, the information provided is so minimal that it is doubtful whether users' needs were considered in any detail, in the case of other sign language dictionaries, considerable efforts were made to identify what information the user groups needed and how this information should be provided. Moskovitz (1994), for example, carried out a detailed pre-compilation survey of 138 potential users of a Dictionary of New Zealand Sign Language (Kennedy et al., 1997). The survey, which was followed up with one-hour interviews with a representative sample of the potential user groups, influenced design decisions such as the use of line drawings instead of photographs, and content decisions such as the inclusion of grammatical information about both the sign and any English glosses, age variation, and mnemonics to aid in the production of signs.

A similar survey (McKee \& Pivac Alexander, 2008) of 301 potential users was carried out at the start of the project to create the ODNZSL. This survey specifically asked users to identify what they might use an online dictionary for, and how they might carry out a search for information, as well as asking about the contents of dictionary entries and technical details about their internet access. Again, the responses directly influenced the eventual inclusion of particular features in the ODNZSL. 


\subsubsection{Multifunctionality}

As previously mentioned, there are usually scant resources available for dictionary making in lesser-resourced communities. Sign language dictionaries have many different kinds of potential users, but the total number of users is relatively small and this means that sign language dictionaries are usually not feasible as commercial projects without additional sponsorship (Wilcox, 2003). As a result, a single dictionary generally has to fulfill multiple functions and has to try to meet both encoding and decoding needs of various user groups (Prinsloo, 2012). Given the exceptionally wide-ranging intended user groups for some sign language dictionaries-Schmaling (2012) observed that "sometimes the target group is defined so widely that the dictionary is basically aimed at anyone..." (p. 249)—it is likely that different purposes and user needs will at times be in conflict.

\subsection{Sign language dictionary types}

In summary, sign language dictionaries try to achieve multiple goals and meet the needs of a broad range of users, within the constraints imposed by the lack of a writing system and the limited availability of resources. We can envisage these issues as opposing influences: whereas diverse purposes and user needs would be best met by a diverse set of dictionary types, the constraints on resources and limitations on representing signs tend to result in a similar type of dictionary with little variation in format. Electronic sign language dictionaries have overcome some of the difficulties associated with the lack of a writing system and have the potential to be tailored to different situations and users, blending aspects of different dictionary types. However, Prinsloo (2012), amongst others, observed that many electronic dictionaries have not made full use of this potential. Instead, these dictionaries are often straight transfers of the same information and format of existing print dictionaries. Sign language dictionaries have been modelled on existing dictionary types, and decisions made for print dictionaries have sometimes carried over into electronic formats, as Zwitserlood (2010) noted in relation to a dictionary of Sign Language of the Netherlands with both print and online versions (Schermer, Koolhof, Muller, Geuze, \& Vink, 2009).

Kristoffersen and Troelsgård (2012) argued that instead of a classification according to traditional dictionary types, it would be more useful to examine how different user needs can be met by a single (electronic) dictionary. The question of which user needs would be met by definitions will be examined in more detail in Chapter 4 - A user study of an online sign language dictionary. First, a brief examination of dictionary types in relation to sign language 
dictionaries is still required to clarify why definitions have only rarely been a part of sign language dictionaries and to explore the potential for their inclusion in future dictionaries.

\subsection{Dictionary typology: some distinctions}

Most typologies include a caveat that many dictionaries blend aspects of different types (e.g. Zgusta, 1971; Swanepoel, 2003), and Béjoint (2000) went as far as to say that "it is impossible to classify dictionaries in a way that would be both orderly and applicable to all societies." (p. 37). A number of broad distinctions can nevertheless be made.

The most common distinction is between monolingual and bilingual dictionaries. In monolingual dictionaries, the lemmas and their description are in the same language. The description in a monolingual dictionary is most often centred around a definition of the lemma's meaning. By contrast, bilingual dictionaries typically offer translational equivalents of the lemma in another language and do not include definitions.

Zgusta (1971) further classified dictionaries according to:

- the kind of information found in the dictionary: linguistic vs. encyclopaedic;

- whether the dictionary covers the lexicon at a particular point in time or traces the history or origins of words: synchronic vs. diachronic;

- whether the entire lexicon is covered, or particular subsections: general vs. restricted / special;

- whether the dictionary describes only standard, regular, expected usage, with a normative effect, or whether it provides information on any kind of words in the language including obsolete and rare words: standard-descriptive vs. overall-descriptive.

The language direction and kind of information provided also depend on whether the dictionary aims to help users with receptive / decoding or productive / encoding language needs.

Béjoint (200o) added further types of dictionaries aimed at particular user groups, including foreign learners' vs. native speakers' dictionaries, and dictionaries for adults vs. dictionaries for children. Swanepoel's (2003) overview of dictionary typologies also considered differences in macrostructure such as the ordering of entries within the dictionary, and differences in microstructure such as the kind of grammatical information offered within an entry, and the ordering of that information. 
Although there are many dictionary types, for most people, the prototypical dictionary is a monolingual, general purpose dictionary, arranged in alphabetical order (van Sterkenburg, 2003). According to Béjoint (2000), this is

"the one that every household has, that everyone thinks of first when the word dictionary is mentioned, it is the type that is most often bought, most often consulted, the one that plays the most important role in the society that produces it."

\subsubsection{Monolingual vs. bilingual}

Given the predominant role of the monolingual, general purpose dictionary, it is not surprising that the desire for a monolingual sign language dictionary has been expressed frequently. Monolingual dictionaries are especially suited to language description and often have a normative effect (Zgusta, 1971) and these characteristics fit well with the stated aims of language documentation and standardisation common to many sign language dictionaries. As mentioned in Chapter 1 - Introduction, Schermer (2006) considered monolingual dictionaries a necessary part of a full description of the sign language lexicon. Yet, to date no general purpose monolingual dictionaries have been created: a monolingual ASL dictionary has been in development since a prototype was created (Bienvenu, 2003) but has not yet been published.

Sign language dictionaries, especially in print format, can be said to be bilingual by default since the absence of a written form necessitates the use of the spoken / written language as a metalanguage. The multifunctional nature of sign language dictionaries also made the choice of a bilingual format preferable. Stokoe et al. (1965) acknowledged that, even if it was feasible to create a purely monolingual dictionary, the DASL's inclusion of English would make it useful as a translation dictionary. A further reason for the DASL's inclusion of English descriptions was the unique sociolinguistic context of ASL which meant that "the deaf user of ASL ... is also part of the general American culture ...” (p.xxvii).

It is not unusual for initial documentation dictionaries of lesser-resourced languages to be bilingual (Zgusta, 1971; Corris, Manning, Poetsch, \& Simpson, 2002). Just as has been the case for sign languages, dictionary projects for many minority or endangered languages have been initiated by non-native speakers whose first need was for translational equivalents. Since this need is shared by the main user groups of these dictionaries, a bilingual format is a logical choice for a multifunctional dictionary. 
For minority, endangered, or 'not yet stabilised' non-standard languages, there are different perspectives on which language should be the source language of the dictionary. Zgusta (1971) suggested that in cases where a (national) language has not yet developed a standard form, a bilingual dictionary should use an established majority language as the source language, since this most clearly shows 'gaps' in the target language. This approach has been taken by many sign language dictionaries, in particular when those dictionaries were intended for use in educational settings. As was mentioned earlier, any perceived gaps were often filled by dictionary makers coining their own signs.

The opposite argument is made by Corris et al. (2002) who stated that dictionaries for endangered languages should have the endangered language as the source language for two reasons. Firstly, they identified that speakers of the language of the wider community would in the first instance have the most need for understanding words in the endangered language rather than producing words. In other words, they envisaged the first dictionary as a decoding dictionary. Secondly, they referred to the symbolic function of dictionaries and stated that for native speakers, using the endangered language as the source language "gives it a kind of primacy." (p. 331).

The importance of prioritising the (minority) sign language by giving it the position of the source language was also stressed by Brien and Turner (1994). They argued that unless the sign language is taken as the source language, there is a risk that the most culturally bound and idiomatic signs are omitted since they might not have an immediate equivalent in the majority spoken language.

The Dictionary of British Sign Language / English (DBSL/E)(Brien, 1992) was one of the first print dictionaries after the DASL that took a sign language as the source language and that were explicitly organised as bilingual, bidirectional dictionaries. This format required changes in dictionary macrostructure. Where previous sign language dictionaries had been ordered alphabetically by the spoken language gloss, the $D B S L / E$ and a small number of other linguistically descriptive dictionaries took the macrostructure of Stokoe et al.'s DASL as an example and organised entries in a custom order based on the handshape and location parameters of the sign. Bidirectionality was achieved through the use of a separate alphabetical index referring to sign entries by number.

In the $D B S L / E$, the two languages (BSL and English) were deliberately kept separate in the main part of the dictionary: whereas the left-hand page contained photographs and an 
adapted Stokoe notation for each sign lemma, the right-hand page contained a description in English on how to produce the sign, several translational equivalents, and usage notes (see figure 3.1: the large letter F on the right hand page in this figure is a label for the handshape rather than an alphabetical ordering mechanism).
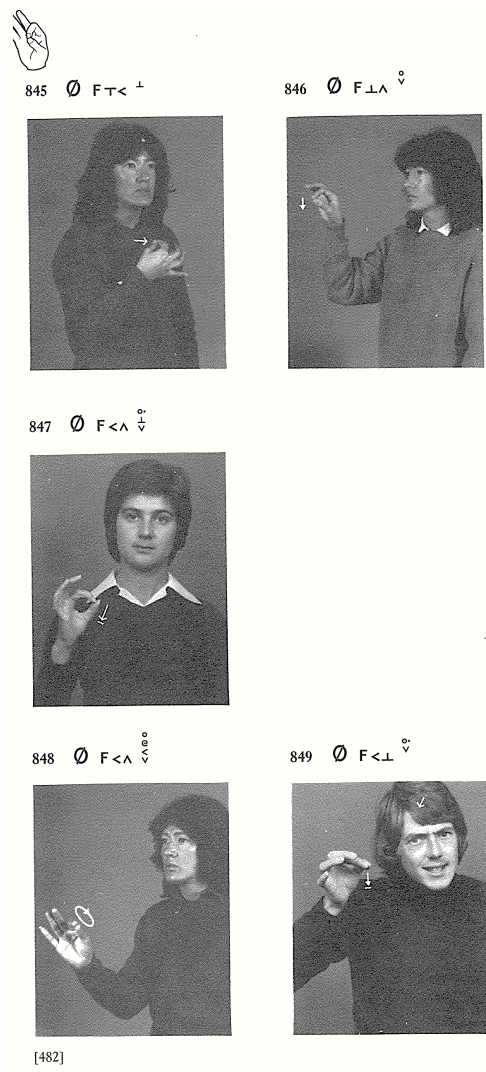

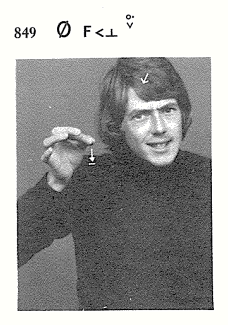

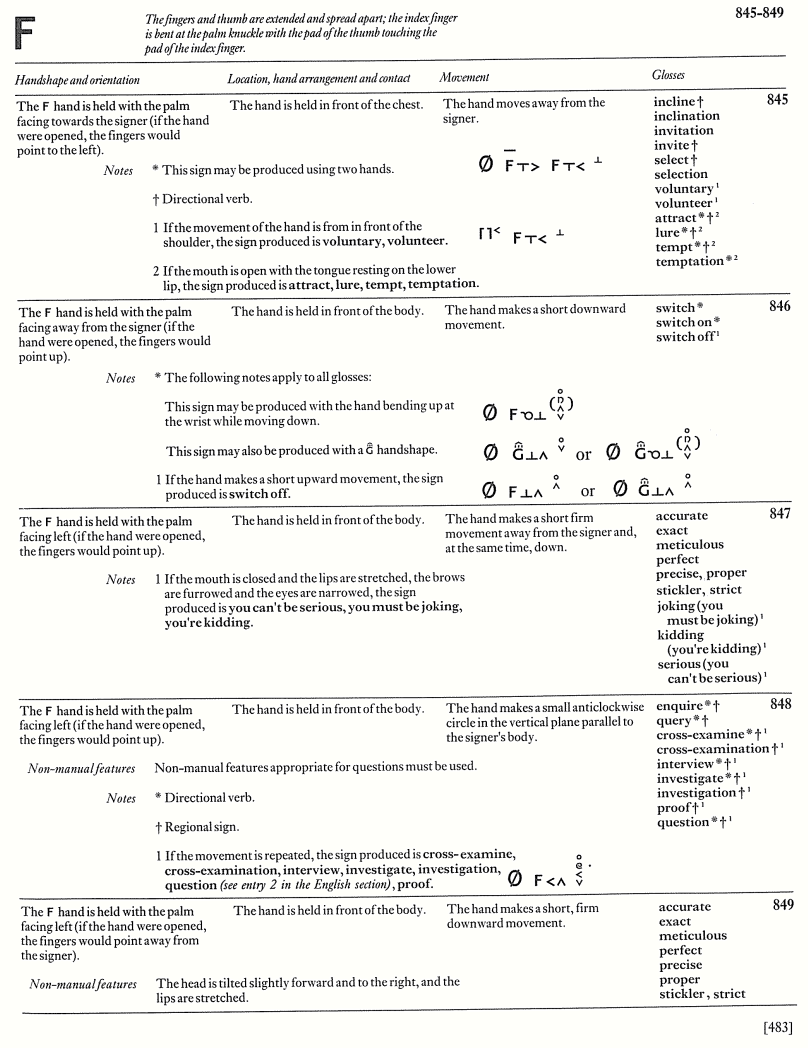

Figure 3.1: An entry in the Dictionary of British Sign Language / English

Uniquely, the $D B S L / E$ also included a Guide to Meaning, where the meaning of BSL signs was described in the target language, English. The resulting dictionary therefore combined elements of monolingual and bilingual formats. Other descriptive sign language dictionaries have not included definitions; in other respects, however, they have also been hybrids between monolingual and bilingual formats. Zwitserlood (2010) noted that the title of a recently published dictionary of Sign Language of the Netherlands implied a monolingual focus. Apart from the $D B S L / E$, which mentions both languages used in the dictionary, it seems to be common practice to refer only to the sign language, perhaps because it is recognised that these dictionaries' function goes beyond the usual translation dictionary. Kristoffersen and Troelsgård (2012) described the online dictionary of Danish Sign Language (Center for Tegnsprog, 2008) as "a monolingual dictionary, which instead of definitions has (searchable) equivalents in another language, Danish, which is also the general metalanguage of the dictionary". 
The use of the spoken language as the metalanguage in addition to its role as a target language of a bilingual dictionary has been recognised as problematic, since it may blur the distinction between the languages. In this respect, Zwitserlood (2010) critiqued the use of glosses to represent sign lemmas in a dictionary of Sign Language of the Netherlands. Schermer and Koolhof (2010) stated user-friendliness as their reason for organising entries in alphabetical order following the Dutch gloss, in preference over an alternative ordering by sign parameter. However, Zwitserlood (2010) pointed out that the gloss in this structure is considered to be equivalent with the sign, rather than being interpreted as one of several possible translations of a sign in the target language. In sign language dictionaries, then, the well-documented problem of anisomorphism or non-equivalence of two words paired in a bilingual dictionary (e.g. Zgusta, 1971; Duval, 2008) is compounded by the lack of an independent representation of the sign lemma's meaning. The inclusion of multiple glosses in some dictionaries, including the ODNZSL, partly mitigates against this issue.

Brien and Turner (1994) recognised that the DBSL/E's Guide to Meaning, written in English text, was a compromise towards a fuller semantic description of sign lemmas. When it became possible to record and store longer videos, the team responsible for the $D B S L / E$ created a pilot CD-ROM Dictionary of Deaf Community and Culture (Brien, Brennan, Collins, Reed, \& Thoutenhoofd, 1997). This CD-ROM demonstrated the potential to create multipurpose dictionaries where the sign language is available as a metalanguage throughout, from the menus and search facilities through to signed definitions. This potential has not yet been completely realised in subsequent electronic dictionaries. The reasons for choosing a bilingual rather than a monolingual dictionary format are still valid in the present day, since resource constraints are similar and this format suits the most user groups. However, a purely bilingual format, where spoken language equivalents are the only semantic description of sign lemmas, may be descriptively inadequate (as will be discussed further in section 5.2 Equivalents or glosses).

\subsubsection{Linguistic vs. encyclopaedic}

Although the focus of sign language dictionaries has mainly been on the documentation of linguistic information about sign lemmas, a case can be made for the inclusion of encyclopaedic information for certain entries. Encyclopaedic entries can highlight culturally significant events and concepts and therefore enhance sign language learners' understanding of the Deaf community and culture. Examples of encyclopaedic information from the BSL CD-ROM Dictionary of Deaf Community and Culture (Brien et al., 1997) can be found in 
entries about 'Deaf poetry' and the 'Congress of Milan' (an important event in Deaf culture since this congress banned the use of sign language in education).

Technical sign language dictionaries may also benefit from the inclusion of encyclopaedic information, since further (non-linguistic) knowledge about the technical concepts would enhance Deaf users' understanding, especially when signs for the concept have been newly coined and will therefore not be familiar. For example, the astronomy section of the online British Sign Language Glossaries of Curriculum Terms (Scottish Sensory Centre, n.d.) includes elements such as an interactive map of the solar system, with links to the sign entries for each of the planets.

The inclusion of broader world knowledge through hyperlinks or layered definitions in electronic dictionaries has been proposed by Fillmore (2003). Fillmore argued that the distinction between linguistic and encyclopaedic knowledge is artificial since we have to call on wider 'frames' of world knowledge to interpret linguistic utterances. Dictionaries, according to Fillmore, could help non-native users to understand a foreign language better by providing them with access to the cultural frames that are invoked when a word is used by native speakers. McKee and McKee (2013) discussed the role of corpus-derived example sentences in conveying such culturally rich information, but also saw an opportunity for future development of encyclopaedic-style definitions for sign language dictionaries. Folk definitions originating from native sign language users could provide a foundation for such developments.

\subsubsection{Synchronic vs. diachronic}

Many sign languages are relatively 'young' and for those sign languages that are known to have existed for a longer time, few historical texts survive. Diachronic dictionaries have therefore been rare. A recent historical and etymological dictionary of American Sign Language (Shaw \& Delaporte, 2014) contained around 500 signs, showing that even in the case of a relatively well-documented sign language, historical evidence of use is not consistently available for many signs.

General synchronic sign language dictionaries can and should play a part in documenting language change (Wilcox, 2003) by providing evidence about the form and use of signs, including variants, at a particular point in time. Later dictionaries can then build on this evidence to create a diachronic record. 


\subsubsection{General vs. restricted}

As was noted in section 3.1 - History and purposes of sign language dictionaries, increased participation of Deaf people in education and in new areas of employment has led to a rapid expansion of vocabulary in certain areas. While some general purpose dictionaries include such recently established vocabulary alongside core sign lemmas, subject-specific and technical dictionaries, both in print and especially in electronic form, have become common for sign languages with a longer history of use.

Technical sign language dictionaries share a bilingual format with general purpose dictionaries; however, in technical dictionaries the spoken language is the source language. Alphabetical ordering or a search method by written word in this case suits the dictionary's purpose: Deaf users' decoding needs (to understand the spoken / written language term), and sign language interpreters' and educators' encoding needs (to express a known technical concept in the sign language) are met by this format. The German Sign Language Fachgebärdenlexikon Gesundheit und Pflege (Konrad et al., 2007) also offers advanced search possibilities, including a search by sign features.

Definitions have been more common in technical dictionaries than in general purpose dictionaries. For example, ArtSigns, an early online sign language glossary for art and design (University of Wolverhampton, n.d.) included written English definitions, supplementary encyclopaedic material, and hyperlinks to related terms. More recently, several online subject glossaries and dictionaries have also included both written and signed definitions including encyclopaedic material: for example, the British Sign Language Glossaries of Curriculum Terms (Scottish Sensory Centre, n.d.) and the Swiss-German Sign Language / German Gebärden-Fachlexikon (Boyes Braem, 2009).

While these definitions offer a useful comparison to the proposed folk definitions that are the subject of this thesis, they are different in nature. In technical dictionaries, the object of the definition is to explain the meaning of the unfamiliar spoken language term to a Deaf dictionary user, using the sign language as the target language. The explanations may include encyclopaedic knowledge as well as linguistic knowledge, and where a sign equivalent has been newly coined, the explanation may focus on features of the sign that make it a suitable choice to represent the technical concept. An example of this definition style can be found in the video definition for 'Neptune' in the BSL Glossaries of Curriculum Terms: http:// www.ssc.education.ed.ac.uk/BSL/astronomy/neptuned.html (Scottish Sensory Centre, n.d.). 
Definitions in a general sign language dictionary, by contrast, would have the meaning of the sign lemma as their object and would aim to make explicit the linguistic knowledge that native users possess about the sign lemma's semantics and use.

\subsubsection{Standard-descriptive vs. overall-descriptive}

According to Zgusta (1971), standard-descriptive dictionaries, also referred to as 'academic dictionaries', focus on the standard national language and describe its regular use at the time of compilation. Although they do not necessarily have prescriptive aims, by their focus on standard usage these dictionaries are normative in character. Overall-descriptive or 'reference dictionaries' describe the wider lexicon, for example including dialectal and regional variation and archaic forms.

As has been stated earlier, sign languages are comparatively 'young' languages that experience a great deal of both synchronic and diachronic variation because of disrupted transmission, restricted domains of usage until very recently, and the lack of a written form. Since there is therefore no standard to describe, it is not possible to create a standard-descriptive sign language dictionary. Overall-descriptive approaches are also problematic because of the sheer extent of lexical variation. While some sign language dictionaries, such as the Auslan Dictionary (Johnston, 1989 a) explicitly indicate regional variants, in many cases evidence for particular variation patterns is scarce (McKee \& McKee, 2011). Decisions for sign language lexicographers therefore include not only which variants to document, but also which forms are included as citation forms and which as variants.

The competing drives towards standardisation and the documentation of variation were already discussed in section 3.1 - History and purposes of sign language dictionaries. One further issue to raise is the interaction of these dictionary types with users' needs and expectations. Zgusta (1971) noted that overall-descriptive dictionaries are usually decoding dictionaries only, supporting dictionary users who are not native users of a particular variant or archaic term to look up its meaning. These users would not normally seek to produce these terms themselves, nor are these dictionaries intended for native users: as Zgusta pointed out, "a man who speaks the local patois of a village will hardly feel any uncertainty about the usage of the respective expressions which are so very much bound to concrete situations" (p. 212). Standard-descriptive dictionaries, on the other hand, serve both decoding and encoding needs for language learners and native users alike. 
Several factors may lead the users of sign language dictionaries to expect that the dictionary they are using is a standard-descriptive, hence normative, dictionary. Firstly, we have seen that many forewords mention multiple user groups including Deaf native sign language users as well as hearing learners. Secondly, the title of the dictionary nearly always includes a national name of the sign language (e.g. 'New Zealand Sign Language'), suggesting to learners that they will find information about a single standard language. Finally, because of the scarcity of research evidence, individual entries may contain little information about variation and the ordering of semantically equivalent variants in the dictionary may not be transparent to the user.

\subsubsection{Macrostructure}

In the previous section it was shown that the choice of a bilingual format with a sign language as the source language affected the macrostructure of some sign language dictionaries. Ordering by the handshape and location parameters of the sign, while it has the advantage of representing the sign language on its own terms, is less user-friendly (Schermer \& Koolhof, 2010) and challenging for a novice dictionary user to navigate (McKee \& McKee, 2013).

Alternatives such as thematic ordering have also been explored in some print sign language dictionaries. An early dictionary of Sign Language of the Netherlands, aimed primarily at children (Schermer, Harder, \& Bos, 1988) was issued in a ring binder with entries on individual cards to enable custom ordering.

Ordering issues are largely avoided in electronic dictionaries, where users can enter search criteria directly. However, traditional searches by written word will only be useful if the dictionary user already knows the meaning of a sign. To look up an unfamiliar sign by its form, alternative search methods are required. A small number of electronic dictionaries have implemented the facility to search by sign parameters: for example, dictionaries of Danish Sign Language (Center for Tegnsprog, 2008), Finnish Sign Language (Kuurojen Liitto, 2003), and New Zealand Sign Language (McKee et al., 2011) (See figure 4.2 in the next chapter). Further searches, for example by theme or by grammatical or usage characteristics of the sign, allow electronic dictionaries to be tailored more closely to users' needs.

\subsubsection{Microstructure}

Reasons that sign language dictionaries generally provide less information about the lemma have been mentioned previously. Nevertheless, entries in many general descriptive sign 
language dictionaries do provide a range of information in addition to representing the sign's form and one or more spoken language translational equivalents. Zwitserlood, Kristoffersen and Troelsgård (2013, pp. 269-270) listed the following additional aspects that may be covered in the sign language dictionary microstructure:

- information about mouth movements if these typically accompany the sign;

- information about form variants, for instance rendered as additional video clips;

- $\quad$ parts of speech;

- morphological information (inflection and derivation);

- description of the use of signs where the meaning or function cannot be rendered satisfactorily through a written language equivalent;

- definitions of sign meanings;

- $\quad$ cross-references to sign synonyms, antonyms, etc.;

- information about usage restrictions, like region or age-specific use of the sign;

- example sentences.

While space considerations often prevented the inclusion of much information in print dictionaries, electronic dictionaries are able to provide additional video material and hyperlinks. Although in principle, entries in electronic dictionaries can be very long since users can scroll down, Zwitserlood et al. noted that multiple videos and links on one screen can become visually overwhelming. The amount of information presented in an entry is an issue common to all dictionaries. Nielsen (2008) discussed the 'information cost' associated with both searching for a lemma and understanding the information given in a dictionary entry and recommended that this cost be kept as low as possible. Users tend to ignore information that is perceived as too complicated and only look as far as they need to in order to identify the item they think is equivalent (Tomasczcyk, 1971; quoted in Béjoint, 2000). The total information cost of an entry, then, needs to be borne in mind as a factor in the decision whether signed definitions are a useful addition to the microstructure of sign language dictionaries.

\subsubsection{Electronic sign language dictionaries}

Throughout this section, electronic sign language dictionaries have been mentioned alongside print dictionaries. The increasing capabilities of computers have influenced lexicography considerably. De Schryver (2003) referred to three phases in electronic 
lexicography: firstly, computer-assisted (paper) lexicography, secondly, transfer of existing paper dictionaries to an electronic format, and finally, electronic dictionaries in their own right. A number of print sign language dictionaries have used computerised databases to store data since the late 1980s (for example, the Dictionary of British Sign Language/English and the Dictionary of New Zealand Sign Language). Wilcox (2003) described an early attempt to transfer the content of the Dictionary of American Sign Language on Linguistic Principles to a multimedia CD-ROM format; however, for a number of reasons this project was never completed. The direct transfer of an existing paper dictionary to an electronic format without further modifications has not been common in the case of sign language dictionaries: since the advantages of using video materials were recognised early on, moving to this format necessitated as a minimum the filming of citation forms for each lemma. There have, however, been concurrent electronic versions of existing print dictionaries, either on CDROM (e.g. Sternberg, 1996) or online (e.g. Schermer et al., 2009) and offering features such as additional search functions, video clips and examples. Other electronic dictionary projects have extensively reviewed and added to the data from existing print dictionaries (e.g. Troelsgård \& Kristoffersen, 2008; McKee \& McKee, 2013).

Modern electronic sign language dictionaries can be found in several formats. Firstly, there are CD-ROMs or DVD-ROMs that may accompany print versions or be offered as a stand alone resource. Secondly, many sign language dictionaries are available online, either as paid or freely available resources. Lastly, there are apps designed to run on mobile devices or tablet computers.

The most comprehensive discussion of electronic sign language lexicography to date can be found in Kristoffersen and Troelsgård (2012). This work summarised the following features that make electronic media particularly apt for representing sign languages:

- hyperlinks and multimedia;

- $\quad$ search facilities (including bidirectional search options);

- flexibility of space;

- $\quad$ meeting diverse user needs.

As was found to be the case with dictionaries in print form, there is great variety in the extent to which these features are exploited in electronic sign language dictionaries. Available dictionaries range from simple, short, unidirectional glossaries in which a search by word retrieves a video of a sign in isolation, to comprehensive general descriptive bidirectional 
dictionaries with multiple search methods, a detailed microstructure including multiple translational equivalents, example sentences, and links to other relevant information. The Online Dictionary of New Zealand Sign Language (ODNZSL) (McKee et al., 2011) is an example of a modern comprehensive online dictionary that includes many of these features and it will be used as a case study throughout this thesis. A full description of the structure and content of the ODNZSL will be given in section 4.5.1 - Materials: The ODNZSL.

Contemporary online sign language dictionaries are now available for a wide range of sign languages. In addition to the dictionaries of Danish Sign Language, Finnish Sign Language, and New Zealand Sign Language that have already been mentioned, there are also online dictionaries of Flemish Sign Language (van Herreweghe, Slembrouck, \& Vermeerbergen, 2004); Auslan (Johnston, 2014); British Sign Language (Fenlon et al., 2014); Estonian Sign Language (Eesti Keele Instituut, n.d.); Czech Sign Language (Müller, n.d.) and Indian Sign Language (FDMSE, n.d.). Sophisticated technical online dictionaries have also been published in the past few decades, for example the previously mentioned Fachgebärdenlexikon Gesundheit und Pflege (Konrad et al., 2007). These dictionaries have been created by teams of Deaf and hearing researchers and their content is based on extensive linguistic research. As well as the features mentioned above, such modern online dictionaries also include new kinds of information such as links to an external spoken language dictionary, encyclopaedic information, other learning resources and exercises, or a vocabulary sheet function where lemmas can be saved and printed out.

Although some online sign language dictionaries already make comparatively full use of the electronic medium, Kristoffersen and Troelsgård (2012) identified further challenges for electronic sign language lexicography. These challenges include:

- exploiting the potential of IT to tailor dictionary content and presentation to individual user needs;

- using the sign language as a metalanguage in the dictionary;

- integrating dictionary content further with other electronic resources;

- adding new information, especially better semantic description of signs through definitions

- expanding and utilising sign language corpora;

- finding out how dictionaries are used in practice through usability studies. 
The last three challenges are particularly relevant to the current thesis. In line with Kristoffersen and Troelsgård's argument that definitions are an "obvious enhancement of comprehensive sign language dictionaries" (2012, p. 312), I will investigate the potential of elicited folk definitions to fulfill this role. It will be argued in the next section that sign language corpora, while providing guidance on the meaning and use of signs in some instances, are not yet sufficiently developed to carry out a semantic analysis of many lexical items. Elicited folk definitions may be added to existing corpora to provide complementary data on sign meaning. Finally, although the electronic medium now provides the opportunity to include signed definitions, the effectiveness of this medium and the extent to which the needs of different users are presently met have not been assessed. A study of the users of an online dictionary is therefore an essential step in the research proposed in this thesis.

\subsubsection{Corpora and corpus-based lexicography}

One of the greatest impacts of electronic technology on lexicography has been the creation of digital corpora. While collections of written texts had been used in lexicography since the $18^{\text {th }}$ century, such early corpora were usually created from a small set of texts chosen by the lexicographer to confirm their own intuitions about already selected lemmas and to provide illustrative quotes. (Béjoint, 2000). Systematic analysis of large-scale digital corpora was first employed in the creation of the Collins COBUILD English Language Dictionary (Sinclair, 1987 a). This dictionary used corpus information to inform lemma selection based on lexical frequency and to provide objective evidence of authentic language use. The COBUILD's use of corpus-based evidence has been hugely influential, although there are ongoing debates over the merits of corpus-based examples as opposed to purposely created illustrative examples (e.g. Fox, 1987; Cowie, 1989) and over the particular style of full-sentence definition that was implemented to reflect authentic language use (e.g. Rundell, 2006). These debates will be discussed in Chapter 5 - Evaluating the user need for definitions and Chapter 6 - Folk definitions respectively, since they are relevant to the application of folk definitions to sign language dictionaries. There is general agreement, however, that corpus analysis provides far more reliable evidence of language use than native speaker intuition.

While corpora for spoken languages are continuously increasing in size and range, sign language corpora are still at an early stage of development. There are both sociolinguistic and technical reasons that sign language corpora are not expected to reach the size and capabilities of spoken language corpora in the foreseeable future. Firstly, the size of sign language corpora will be constrained because there are fewer texts available. In the absence of 
a print culture, signed texts have been restricted to film or video recordings. Until recently, such recordings were not generally publicly available. Many sign language corpora are therefore largely based on elicited material gathered either for the specific purpose of creating a dictionary, or for other linguistic research (as can be seen in the data descriptions in the inventory of sign language corpora maintained by Konrad, 2012). More signed texts are now becoming available as sign languages are increasingly used in public domains: for example, in television programmes and information videos targeted at sign language users, and through increased shared video communication through social media. However, the number of texts for individual sign languages is likely to remain relatively small.

Secondly, the automated processing of texts that is possible for spoken language corpora is not feasible for video recordings. To create searchable corpora, video texts have to be coded with annotations and transcriptions. Early sign language corpora such as the Wellington Corpus of NZSL (McKee \& Kennedy, 2006) used spoken language glosses to create transcriptions that were not linked to the original video material. The drawbacks of glossing were described in section 3.2.1 - Representing signs without a written form. Modern software such as ELAN (Max Planck Institute for Psycholinguistics Language Archiving Technology Group, 2009) and iLex (University of Hamburg: see e.g. Hanke, 2002) enables time-linked coding using multiple tiers of information (including phonological sign notations), but this remains a very time-intensive process that requires skilled annotators. Most current sign language corpora are therefore only partly completed. Fenlon, Schembri, Johnston, and Cormier (2015) stated that one of the larger corpora, the AUSLAN Corpus, currently contains around 105,000 fully annotated tokens. It is expected that full annotation of the remaining texts will require another five years at least.

Thirdly, as was described in section 2.2 - Linguistic structure and the lexicon, there are still uncertainties around fundamental aspects of sign language linguistics such as the question of what constitutes a sign lexeme, which word classes are found in sign languages, and the categorisation of e.g. polycomponential signs, indices, and mouthing. Such questions affect consistent lemmatisation of sign language corpora (Fenlon, Cormier, \& Schembri, 2015). Often, sign language corpora are developed in parallel with lexical databases, with each project relying on input from the other (e.g. Crasborn, van der Hulst, \& van der Kooij, 20o1; Johnston, 2008; Mesch \& Wallin, 2012). 
In spite of these shortcomings, sign language corpora are increasingly used to inform aspects of sign language lexicography. Konrad's (2012) inventory listed 34 corpus projects for 16 sign languages: nine of these projects had lexicography as one of their aims. Lexical frequency data from the 100,00o token Wellington Corpus of NZSL (McKee \& Kennedy, 2006) informed lemma selection for a Concise Dictionary of NZSL (Kennedy et al., 2002). Corpus data is also used extensively in a number of online dictionaries (e.g. Prillwitz et al., 2008; Mesch \& Wallin, 2012; McKee \& McKee, 2013). Lexical databases created for the purpose of corpus development have partly been made public in online dictionary form as the Auslan and BSL SignBank projects (Johnston, 2014; Fenlon et al., 2014).

Of particular note is the inclusion of corpus-derived example sentences in online sign language dictionaries. Both the Danish Sign Language Dictionary (Center for Tegnsprog, 2008) and the ODNZSL (McKee et al., 2011) include signed examples on video accompanied by a translation into the spoken language and a series of hyperlinked glosses linking to other sign lemmas used in the example. Such example sentences can help users understand both the meaning and use of signs in context, according to McKee and McKee (2013). This raises the question whether corpus-derived example sentences (combined with translational equivalents in the spoken language) actually provide dictionary users with sufficient insight into the meaning of signs, or whether there is a need for separate definitions. As previously mentioned, McKee and McKee certainly see the potential for (encyclopaedic) definitions for particular culturally rich signs. They also stated that due to the limited size of the corpus, suitable examples illustrating typical use could only be found for approximately $50 \%$ of the headwords. Further data on sign meaning and/or usage would therefore be required for authentic examples for all lemmas.

In conclusion, although sign language corpora are of great benefit to the development of sign language dictionaries, they are not currently large or complete enough to enable a full, systematic analysis of sign language use. It is likely that dictionary compilers will continue to rely in part on other sources of data, such as existing lemma lists and consultation with native sign language users. Elicited folk definitions from a range of sign language users will be a valuable addition to data about sign meaning for lexicographers, irrespective of whether they prove to be useful or necessary as additions to the dictionary entry. 


\subsection{Conclusion}

The aim of this chapter was to explain why signed definitions have rarely been a feature of sign language dictionaries to date, and to explore the potential for their inclusion in future dictionaries.

In this chapter I have therefore reviewed the history, purposes, format and structure of sign language dictionaries to date. Reasons that signed definitions have been rare include the predominantly bilingual format of sign language dictionaries, which itself has arisen from difficulties in representing signs without an orthography, the multiple functions these dictionaries have to fulfill, and an initial focus on hearing L2 user groups.

The review of the literature found that the lack of definitions, and of semantic description of signs in general, was recognised as problematic. Opportunities to include definitions are now offered by electronic media. In spite of this potential, recent comprehensive online dictionaries have not included definitions.

The increasing role of corpora in sign language lexicography was found to be an important factor; however, for the foreseeable future it is likely that elicited data will play a large part in the creation of sign language dictionaries.

The conclusion that can be drawn from this literature review is that signed folk definitions have two contributions to make to sign language lexicography: firstly, as a method for gathering specific data about sign meaning and use, as a complement to corpus data; and secondly, as a basis for signed definitions in the dictionary entry.

With respect to this second contribution, it has to be acknowledged again that sign language lexicography operates under considerable resource constraints and that there are a number of other descriptive challenges outstanding that might take priority over the inclusion of signed definitions. Before going on to examine the format of signed folk definitions, it is therefore important to evaluate whether signed definitions will be beneficial to the various user groups of sign language dictionaries, and in what circumstances. The next two chapters will address this question. Chapter 4 - A user study of an online sign language dictionary describes a study of users of the ODNZSL and Chapter 5 - Evaluating the user need for definitions examines the content of the ODNZSL to consider which types of sign lexeme are potentially problematic for these users. 


\section{Chapter 4: A user study of an online sign language dictionary}

\section{Introduction}

The previous chapter outlined the development of sign language dictionaries and the social and linguistic factors that have shaped their format. Current electronic sign language dictionaries were found to have overcome some of the earlier challenges of representing signs without a written form and to have the potential to include signed definitions. For a number of reasons these dictionaries remain largely bilingual and multifunctional, having to meet the diverse dictionary needs of several envisaged user groups.

Dictionary users have become increasingly central to lexicographical research (e.g. Nesi, 2013 b). Hartmann (1989) outlined a number of research principles to govern this area of research. One of these principles is that "an analysis of users' needs should precede dictionary design" (p. 103). The main proposal of this thesis-to add signed definitions as a new element of sign language dictionaries-has implications for the (re-)design of the dictionary entry. It is therefore important first to establish a baseline of current dictionary use, in order to identify what user needs signed definitions might fulfil, and for which users.

Since empirical studies of sign language dictionary use have been scarce to date, I determined to carry out a user study as part of the thesis. In this chapter I will report on this preliminary work, which takes the form of a case study of the Online Dictionary of New Zealand Sign Language (ODNZSL). The chapter will begin with a short overview of user studies to date and their relevance to sign language dictionaries. A summary of studies specific to sign language dictionaries will indicate what questions about user behaviour still remain to be answered. Based on this overview, research questions for the user study will be articulated. Next, an evaluation of the literature outlining a methodology for user studies will lead to my selection of two complementary methods for the current study: log files, and a Think Aloud Protocol (TAP). After describing these methods and the study procedure, the remainder of the chapter will present and discuss the results of the study and implications for the main thesis. 


\subsection{User studies in lexicography}

The motivation and purpose for placing the user at the centre of dictionary developments are clear and widely acknowledged. Hartmann (1983), for example, stated that "all dictionaries are motivated by and judged against the lexical needs of those who consult them" (p. 9). Nesi (2013 b) articulated the aim of all user studies simply as “... to discover ways to increase the success of dictionary consultation" (p. 62). In spite of this clear purpose, Nesi found that there are still large gaps in our knowledge of how dictionaries are consulted.

Several authors have called for a more systematic approach to user studies, with the aim of identifying the relevant variables and principles and outlining a suitable methodology. The work of Wiegand (e.g. 1977; 1998) in formulating a user-focused theory of lexicography continues to exert a strong influence over the field. While Wiegand's theoretical work is beyond the scope of the current chapter, his call for a 'sociology of the dictionary user' was taken up by Hartmann (1989), who posed the following areas of enquiry that would form part of such a sociology:

- history of dictionary use;

- the functions of the dictionary;

- dictionary typology;

- the image of the dictionary;

- user needs;

- variations in use;

- reference skills.

In a previous work, Hartmann (1983) had posed four variables that determine dictionary users' needs:

- information (such as meanings, pronunciation, spelling, or facts contained in the dictionary);

- operations (how people use the dictionary);

- $\quad$ users (for example children, teachers, critics...);

- purposes (e.g. extending native language knowledge, learning a foreign language, playing word games, composing a report etc.). 
In a later work, Hartmann (2001) concluded that a complete empirical user sociology was still lacking. In this work, Hartmann included pedagogical lexicography, dictionary awareness and the need for user training as perspectives on dictionary use, and also introduced the category of 'reference needs' or 'activity contexts' in which a user may decide to consult a dictionary. Tono (2003) combined Hartmann's earlier (1989) classification with the inclusion of two further categories: the teaching of dictionary skills, and critical comparisons and reviews of dictionaries. Tono also substituted Hartmann's (1989) "user needs" with Hulstijn and Atkins' (1998) wording: "the attitudes, needs, habits and preferences of dictionary users" (p. 7).

Even within the work of a single author, then, there is already considerable variation in the terminology and classification of user studies. This indicates that an undisputed, comprehensive theory of dictionary use has not yet emerged. In particular, the interrelated concepts of 'user needs', 'dictionary functions' and 'situations of use' are defined differently depending on the theoretical or practical framework that a particular author subscribes to. For the purpose of this study, I will follow the definitions of these terms as outlined by Tarp (2009) within the context of the theory of lexicographical functions (e.g. Bergenholtz \& Tarp, 2003).

\subsubsection{Theory of lexicographical functions}

The theory of lexicographic functions, according to Bergenholtz and Tarp (2003) is situated within lexicography as an independent scientific discipline. The theory states that lexicographic functions are determined by three factors: users, user needs and user situations. These factors are interrelated and bound to a particular context: that is:

"A lexicographic function of a given dictionary is to provide assistance to a specific user group with specific characteristics in order to cover the complex of needs that arise in a specific type of user situation." (p. 176)

Tarp (2009) further expanded on each of these factors, arguing that empirical dictionary studies should identify the type of users, user situations, and user needs involved and also consider the users' usage of a dictionary (i.e. the dictionary consultation) and the degree to which user needs are satisfied (i.e. whether the consultation was successful or not). 


\subsubsection{Users}

Although in an ideal situation, dictionaries would be able to tailor how they present information to individual users, there are practical limitations to this kind of customisation. In reality, dictionary makers have to target larger groups of people with similar user needs. Tarp (2009) therefore refers to types of user. The particular characteristics that make up a user type are not further elaborated by Tarp on this occasion. However, in an earlier work, Bergenholtz and Tarp (2003, p.173) outlined a number of questions that could be asked to create a more detailed user profile:

“1. Which language is their mother tongue?

2. At what level do they master their mother tongue?

3. At what level do they master a foreign language?

4. How are (sic) their experience in translating between the languages in question?

5. What is the level of their general cultural and encyclopaedic knowledge?

6. At what level do they master the special subject field in question?

7. At what level do they master the corresponding LSP [Language for Specific Purposes] in their mother tongue?

8. At what level do they master the corresponding LSP in the foreign language?"

These questions distinguish between native speakers and foreign language learners and also identify the users' different skill levels, as well as their linguistic and general knowledge, whether specialist or generic.

Varantola (2002, p. 33) expressed a similar distinction more succinctly by identifying three broad types of user:

- language learners;

- $\quad$ professional users (for example, translators);

- non-professional users.

Nesi (2013 a) also added age as a factor. The difference between younger and adult learners can partially be expressed by their different skill levels in their native and foreign language and their general knowledge; however, it is likely that school age beginner foreign language learners, for example, will have different dictionary requirements than their adult counterparts at the same level. 


\subsubsection{User situations}

Tarp (2009) defines the user situation as the situation that prompts the dictionary use. He distinguishes this situation from the usage situation, which is the actual dictionary consultation.

Tarp divides user situations into three categories:

- Communicative situations: where users have a problem communicating with another person or persons and they intend to consult a dictionary to solve this problem.

Communicative situations include language production, comprehension, translation, text revision and marking.

- Cognitive situations: where users intend to consult a dictionary to find general cultural, encyclopaedic or linguistic information. Cognitive situations can prompt either systematic or sporadic dictionary use.

- Operative situations: where users want to find out how to consult the dictionary itself, e.g. by reading a manual or how-to section.

The first two categories replace the previous labels of communication-orientated and knowledge-orientated situations referred to by Bergenholtz and Tarp (2003), who consider user situations as the main determinant of a dictionary's function.

\subsubsection{User needs}

A lexicographic user need is essentially a need for information experienced by a particular type of user in a specific situation. Tarp further specifies the nature of user needs through a number of contrasts.

Firstly, Tarp distinguishes between the potential needs of users as envisaged by dictionarymakers, and the actual needs they experience during the dictionary consultation. Empirical research is focused on examining actual user needs.

Secondly, Tarp identifies lexicographic user needs as historical-cultural needs that are likely to change over time and that may take different forms, since dictionaries will have evolved in different ways in different cultures.

Thirdly, Tarp differentiates between recognised and unrecognised needs. Unrecognised needs are an obvious target for lexicographical improvements. However, if either the users or the lexicographers have not recognised that a particular need exists, then no dictionary 
consultation will take place: in the first case, because the user isn't aware that their need could be met by a dictionary, and in the second case, because such a dictionary does not yet exist. Tarp therefore warns that it is not sufficient for user studies to only concentrate on actual dictionary lookups.

A fourth distinction is between subjective and objective user needs; in other words, what users think they are looking for vs. what information they actually need to solve their problem. Empirical studies should focus on objective needs and this has consequences for the chosen methodology, since users will only be able to articulate their subjective needs if asked directly (for example, in a survey or interview).

Fifthly, Tarp acknowledges that some user needs are not genuine needs at all, but rather are generated by marketing and publicity to persuade users of the benefits of a particular dictionary. Although sign language dictionaries have not usually been commercial enterprises, it may well be that the benefits of certain features are unintentionally exaggerated in an attempt to secure funding or to attract larger numbers of users.

Tarp concludes by arguing that user studies should take care to separate the needs that arise before the dictionary is consulted (for example, the need to translate a word into a foreign language) from the usage-related needs during the dictionary consultation (for example, the need to locate a particular word sense quickly).

\subsection{User studies of sign language dictionaries}

There are few studies in the sign language lexicography literature that are explicitly focused on the user. In this section I will refer to two kinds of studies that reveal something about the “attitudes, needs, habits and preferences" (Hulstijn \& Atkins, 1998) of the users of sign language dictionaries. The first type of study aims to identify potential users' needs prior to the compilation of a new sign language dictionary. The second type of study evaluates one or more existing sign language dictionaries to see whether it meets user needs.

\subsubsection{Pre-compilation surveys}

Two examples of pre-compilation surveys of potential dictionary users are relevant to the current study, since they were carried out as part of New Zealand Sign Language dictionary projects. These surveys were previously mentioned in section 3.2.2 - Envisaged user groups. 
The survey and interviews carried out by Moskovitz (1994) prior to the compilation of $A$ Dictionary of New Zealand Sign Language (Kennedy et al., 1997) identified vocabulary learning, increasing NZSL skills, and acting as "a reference book to check words and signs" as major potential dictionary functions. Compared to these communicative purposes, cognitive situations (for example, "browsing the dictionary for enjoyment" or "finding out more about the Deaf world through supplementary information") were rated as less important. A stated preference for the language direction English-NZSL by $90 \%$ of the participants, combined with low numbers of participants stating that they would use the dictionary to look up English words when unsure of their meaning or spelling, indicated that the main user need would be language production in NZSL by non-native users. Survey participants also valued the wider status-enhancing and standardising effects a sign language dictionary might have. The inclusion of (written) definitions was rated as a low priority by the participants.

McKee and Pivac Alexander (2008) surveyed potential users at the beginning of a project to update and supplement the content of the print Dictionary of New Zealand Sign Language and to create an online multimedia resource, the ODNZSL. In contrast with Moskovitz (1994), this survey did not ask participants to identify their purpose for using the dictionary; however, participants indicated the capacity in which they would use the dictionary as, for example, a teacher of NZSL, a learner, an interpreter, a Deaf student, a parent of a deaf child, or staff working in Deaf education. This last group contained the most respondents, followed by NZSL learners.

The electronic format allows for more flexibility in both searching for and presenting dictionary information. An indication that dictionary users appreciate this flexibility comes from the fact that the majority of participants in McKee and Pivac Alexander's survey preferred to have access to all possible options. For example, although there was still a marked preference for the language direction English-NZSL (49\%), 44.5\% of participants indicated that they would use all search options available, i.e. also searching by features of a sign or by topic. Similarly, more than $80 \%$ preferred to include both videos and static drawings of the sign, and more than half of the participants (54.5\%) preferred to include all the possible information categories within the dictionary entry.

Example sentences on video showing the target sign in context were rated the highest priority, followed by meanings, synonyms, grammatical class, information about variation, and finally examples of the English word used in an English sentence. Although 'meanings' 
were rated higher than in Moskovitz' survey, it is not clear whether participants understood this to refer to definitions or to translational equivalents.

A vocabulary sheet function whereby signs from the dictionary can be saved and printed was evaluated positively by $82 \%$ of the participants, and overall more than $80 \%$ of participants indicated that extended learning activities or games were either important or a 'nice to have' feature if project funding allowed. These responses suggest that vocabulary learning and increasing NZSL skills continued to be priorities for potential dictionary users.

A large majority (86\%) of participants were in favour of a proposed online feedback mechanism whereby Deaf people could comment on dictionary content, showing a high interest by native users in the content of the dictionary.

The results of these two pre-compilation surveys were vital in identifying those features that users would value in the respective dictionaries. However, returning to Tarp's (2009) categorisation of user needs, it is clear that such surveys only identify potential, subjective and recognised user needs. Findings from pre-compilation surveys should therefore be compared with empirical evidence of actual dictionary use.

Furthermore, user needs can change rapidly. For example, McKee and Pivac Alexander's survey included questions around technological issues associated with the new electronic format, such as whether participants had access to broadband and whether a DVD should be produced alongside the online version. Even within the three years of the ODNZSL project, this situation had changed considerably with increased internet access and the spread of tablets and mobile devices. It is therefore important to re-assess user needs regularly.

\subsubsection{Evaluations of existing dictionaries}

There are a small number of critical reviews and evaluations of existing sign language dictionaries. Zwitserlood (2010), for example, reviewed a dictionary of Sign Language of the Netherlands. Zwitserlood provided an insight into the usability of the dictionary under review by discussing specific entries where information about the sign (such as glosses or example sentences) was unhelpful to the potential user.

Schmaling (2012) carried out a comparison of African sign language dictionaries. As well as a detailed comparison of these dictionaries in terms of megastructure, macrostructure and microstructure, this review also included observations relevant to dictionary use. For 
example, Schmaling noted that many African sign language dictionaries are not widely distributed and that Deaf people are therefore often not aware of their existence.

Critical reviews such as these can provide further information about user needs. However, reviewers are likely to have expert linguistic or lexicographical knowledge that is not available to the average dictionary user. Therefore, issues highlighted in these reviews may not coincide with objective user needs.

Further explorations of sign language dictionary user needs can be found in a few studies which encouraged dictionary users to provide feedback on an existing dictionary or prototype. McKee and Pivac Alexander's (2008) survey participants had the chance to view a pilot online NZSL dictionary and comment on its features. It is easier for users to comment on dictionary features that they have used in practice, rather than envisaged features with which they have no experience. As a result, comments in this part of the survey were much more specific about aspects of the layout, design and content of the dictionary. Such comments point to the actual needs users experience while consulting the dictionary, even though these needs are reported subjectively after the fact.

Ow (2009) carried out a survey of users of an electronic Malaysian Sign Language dictionary. This survey, however, had some design flaws that may have made the results unreliable. The survey asked participants to rate the dictionary according to a number of leading statements such as "The 500 words in e-Sign Dictionary is sufficient for communication with the deaf and for daily use" but did not allow for further comments. Only 45 participants were surveyed, of whom 30 were recruited from the general public. Only one third of the participants therefore had direct experience of using the dictionary.

In summary, studies about the users of sign language dictionaries so far have mainly identified subjective user needs and have relied on a limited number of methods: surveys and critical reviews.

\subsection{Research questions}

Since the current study is the first empirical study of the ODNZSL, and indeed may be the first study of its kind for any online sign language dictionary, the research questions were deliberately kept broad. The four questions were formulated to focus on the users, user situations, usage situations, and user needs respectively. 
- Who uses the ODNZSL?

- What situations prompt these users to use the dictionary?

- How is this dictionary used, and what kinds of information do users look up?

- Do users have particular problems or issues in using this dictionary?

\subsection{Methodology of user research}

An appropriate methodology for empirical user studies was discussed by Hartmann (2001). Hartmann listed six social science methods that had been employed in dictionary user research:

- critical reviews;

- questionnaires;

- interviews;

- protocols;

- experiments;

- tests.

Several authors drew upon this classification, including Tono (2001), Tarp (2009), Welker (2010) and Nesi (2013 b), with some modifications such as the addition of 'observations' as a method, and the omission of critical reviews. The later studies (Tarp, Welker and Nesi) also included 'log files' as a method that had become available with the advent of online dictionaries. In deciding which of these methods would be most suitable for the current study, I considered the advantages and disadvantages of each method outlined by all these authors, but in particular Tarp (2009).

As was discussed in the previous section, the only methods used in relation to sign language dictionaries have been critical reviews and questionnaires / surveys. Some of the disadvantages of these studies were already mentioned. Although questionnaires have been very common, Tarp questioned the reliability of this method since it elicits subjective answers. Béjoint (2000) also mentioned that respondents were likely to give answers that they thought would be preferred by the researcher. In Deaf communities, a further disadvantage of questionnaires is that they rely on written information that may not be easily understood by some Deaf participants, since average levels of literacy in the Deaf community are lower than in the general population. 
Interviews benefit from the researcher being present in person to explain or clarify questions, and to ensure that participants give 'honest' answers. However, the presence of the interviewer may also influence the participant's actions and this method still elicits subjective answers. Interviews are also time-consuming and are therefore likely to include fewer participants. Nevertheless, Tarp recognised that qualitative, relatively unstructured interviews could complement the data on actual usage gained by other methods such as observations, protocols, or log files.

Observations, protocols, and log files all record actual interactions of users with the dictionary. Observations involve the researcher observing or recording users' interactions with a dictionary and making notes about what they observe. Protocols ask participants to comment, either on paper or orally, on the actions they are taking during a task involving dictionary use. Log files are computer files recording users' interactions with an electronic dictionary. Of these methods, observations and log files are the most reliable and provide objective data. Log files in particular are unobtrusive and able to access large amounts of data, which can be analysed both qualitatively and quantitatively. However, these methods provide no insight into the reasons the user decided to consult the dictionary in the first place (i.e. the user situation). Protocols, on the other hand, can include the context before and after the dictionary consultation but only show the users' subjective needs. Like interviews, protocols are time-consuming and likely to involve fewer participants.

Experiments and tests assess the performance of dictionary users under particular conditions, to evaluate to what extent a dictionary meets user needs. Experiments consist of one or more tests and either compare the results of a control group against the experimental condition, or alternatively compare results pre- and post-test. To be effective, experiments need to isolate external variables and have access to a high number of participants, ideally selected at random. The kind of test may influence the reliability of the results. For example, multiple choice questions are easy to administer but may skew results.

Both Hartmann (2001) and Nesi (2013 b) stated that it was desirable to triangulate results from different studies by using a number of different approaches. Tono (2001) and Hartmann (2001) also emphasised the value of qualitative studies with a limited set of participants and individual case studies to gather in-depth data as a supplement to larger quantitative studies.

Taking into consideration the broad nature of the research questions, the need to gather objective data about sign language dictionary users to compare with what is known about 
their subjective needs, and the benefits of using both qualitative and quantitative methods, I decided on a study design using two methods:

- $\quad \log$ files;

- a think aloud protocol (TAP) with a short follow-up interview.

\subsection{Method}

\subsubsection{Materials: The ODNZSL}

The ODNZSL was chosen as the subject of this user study for three reasons:

1. Representativeness: as previously described, the $O D N Z S L$ is a representative example of modern online dictionaries and contains the features that make it possible in principle to add signed definitions.

2. Availability of data: general website traffic for the ODNZSL has been tracked since its inception in July 2011.

3. Availability of participants: due to my personal involvement with the project to create the ODNZSL, I was familiar with its features and had access to potential users.

The ODNZSL was launched in July 2011 and at the time of this study contained around 4,300 headwords.

A mobile application of the ODNZSL was created by a volunteer (Hewgill, 2013) and made available as a free download on both Apple (iOS) and Android platforms. No analytical data was available for users of the app on iOS, but a total of 7737 downloads on Android devices had taken place by the end of the selected time period (June 2014) and 3,004 of these devices were logged as 'current users' at that time. Although these statistics suggest that a considerable proportion of users were accessing the ODNZSL via the app, the current study did not include app usage in any detail for two reasons. Firstly, the app contained less dictionary content, omitting example sentences and usage notes. At the time of this study, it was also not possible to carry out a search by features of the sign on the app. Secondly, no logged user data was available for analysis beyond the number of overall downloads. 


\subsubsection{Features of the ODNZSL}

\section{The home page}

Figure 4.1 shows the home page of the ODNZSL.

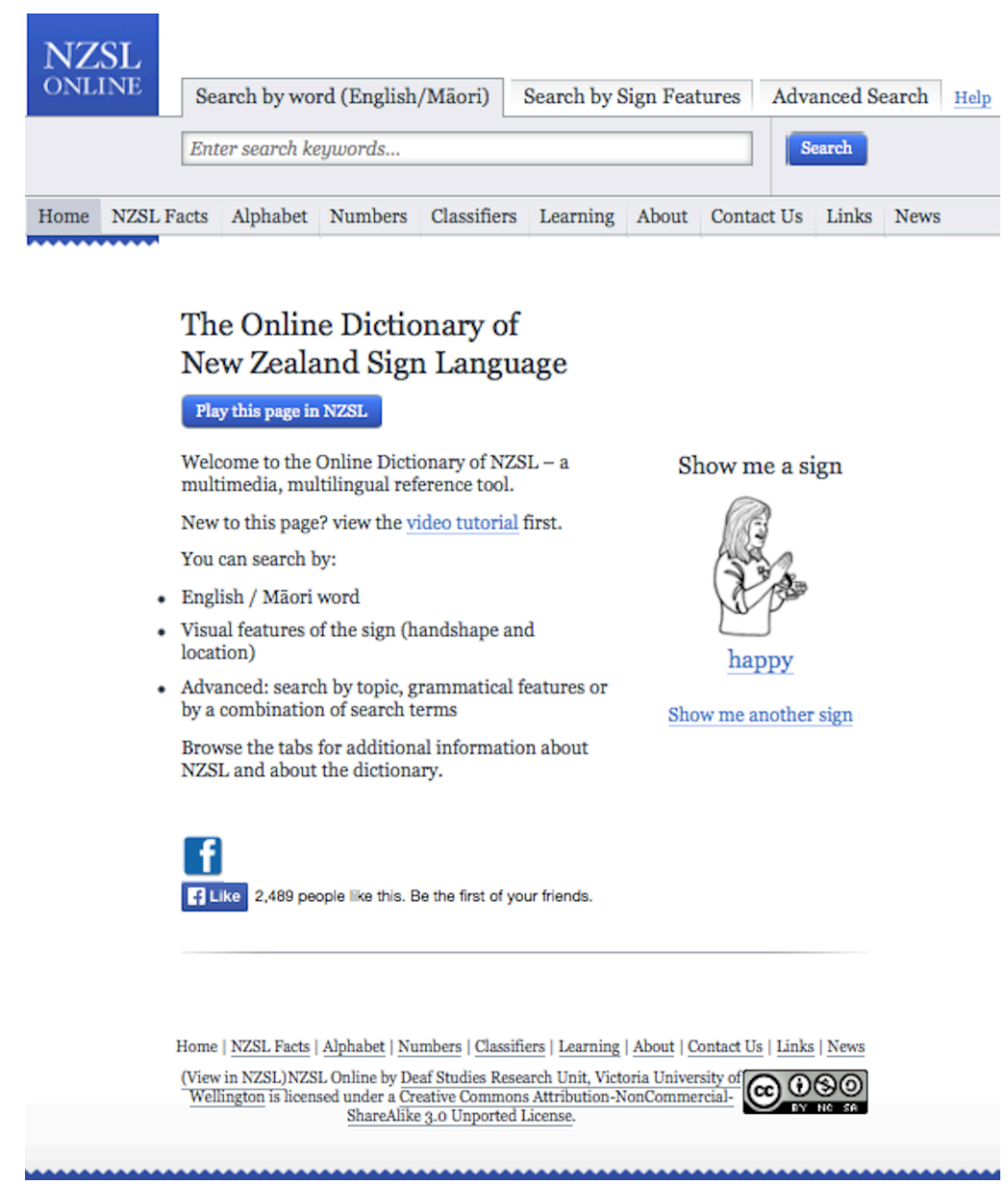

Figure 4.1: The ODNZSL home page

The home page gives access to the front and back matter of the dictionary through a series of tabs, providing background information on NZSL; grammatical information about the number system, fingerspelling alphabet, and classifier / polycomponential signs in NZSL; a help menu which also contains a glossary of terms used in the description of signs in the dictionary; advice for learners with a link to learning exercises; links to relevant organisations; and a contact form which allows users to provide feedback or ask questions.

A 'show me a sign' feature provides a link to a random sign entry, in a similar way to the 'Word of the Day' now provided by some online dictionaries.

By clicking the 'play this page in NZSL' button, the information on the home page and in the tabs can be viewed in video format signed in NZSL. NZSL is therefore used not only as part of the bilingual dictionary structure but also, where possible, as a metalanguage. Te Reo Māori 
translations of each headword were added to the ODNZSL in 2013, so that all three official languages of New Zealand are now represented in the dictionary, although Te Reo Māori is not used as a metalanguage.

\section{Search methods}

Three search methods are available:

- The Search by Word (English / Māori) is a standard search box, which brings up predictive text suggestions of headwords in the dictionary once the user starts typing.

- The Search by Sign Features asks users to select two main phonological features of a sign from a menu of images: the handshape and the location where the sign is produced. (See figure 4.2.)

- The Advanced Search allows for a combination of search criteria from the above two methods, as well as a choice of topics for a thematic search and a list of five usage tags: neologism, archaic, obscene, informal and rare.

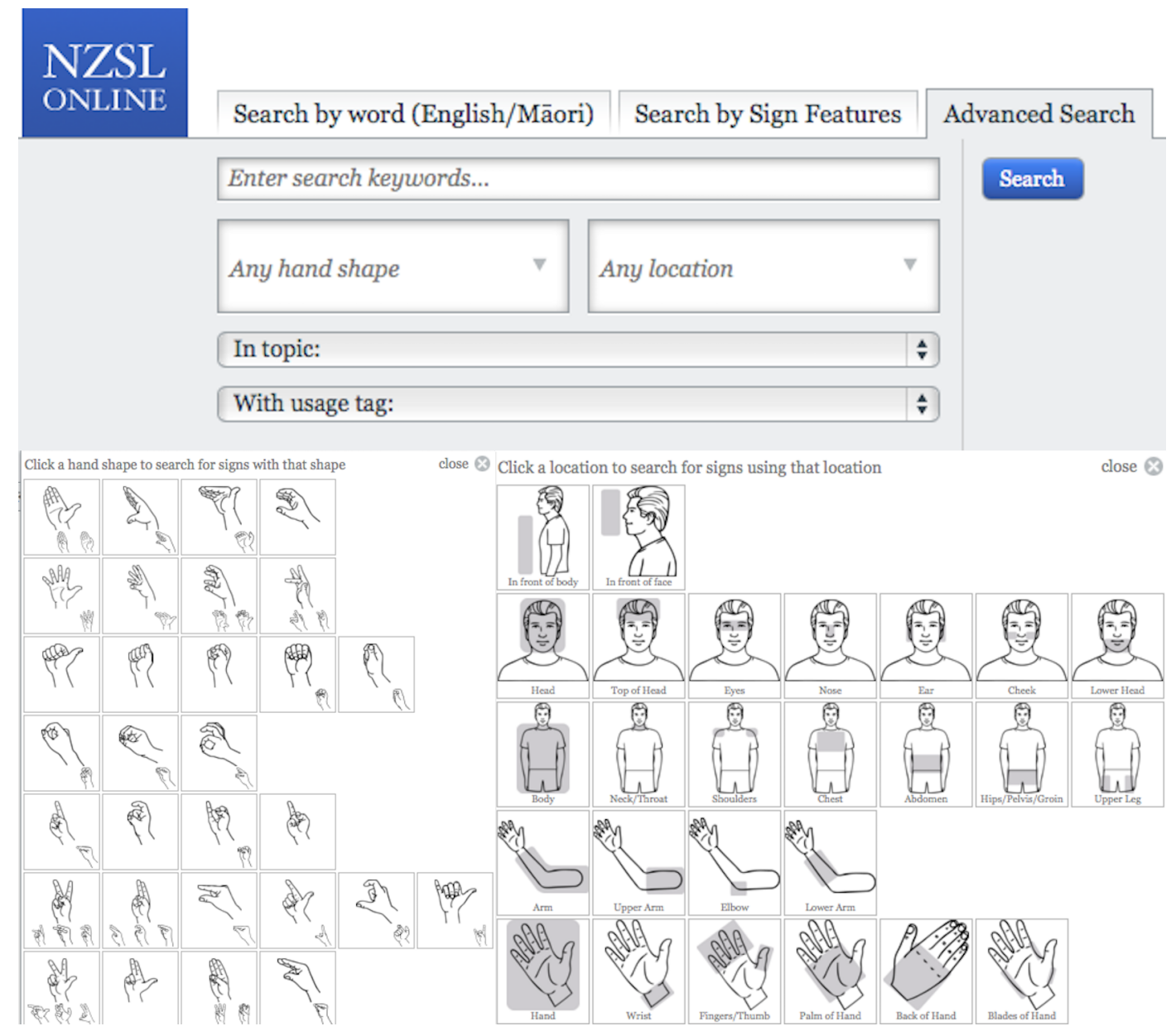

Figure 4.2: Search methods in the ODNZSL 


\section{Search results}

Information displayed in the search results consists of a drawing representing the sign form, followed by glosses in English and Te Reo Māori that capture the main sense(s) of the sign, a series of further translational equivalents in English, and the word class(es) the sign belongs to. Static representations of the sign are used here instead of video files in order to speed up the loading of the search results. Due to the space the drawings take up, results are paginated with a limit of nine results displayed per page (see figure 4.3). Results are displayed in alphabetical order with exact matches for the main gloss displayed first, before exact matches in the translational equivalents and partial matches in both. When there are multiple exact matches, the most frequent sign is displayed first.

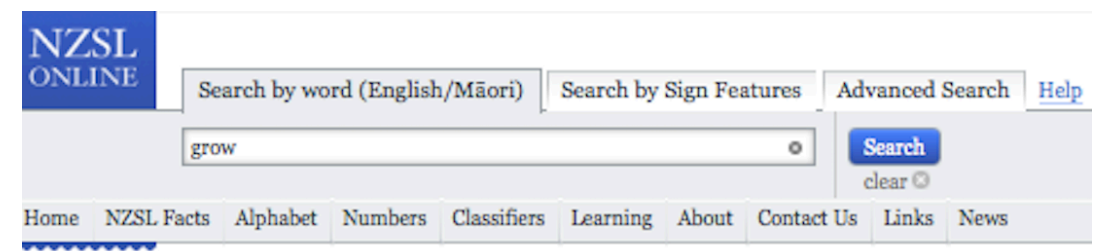

Search results for: "grow"

4 results
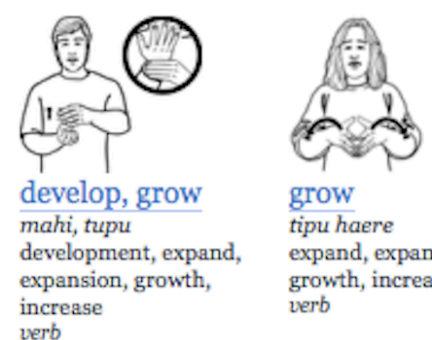

tipu haere

expand, expansion,

growth, increase

verb

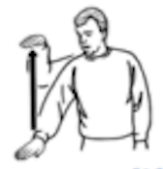

grow up, lifetime

e tipu ake ana, aku rā

katoa

childhood, life

noun

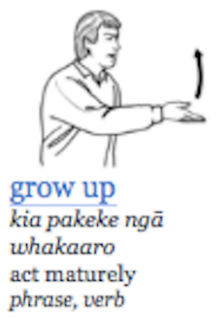

Figure 4.3: Search results display in the ODNZSL 


\section{The dictionary entry}

Figure 4.4 shows the information that is displayed for an individual entry.
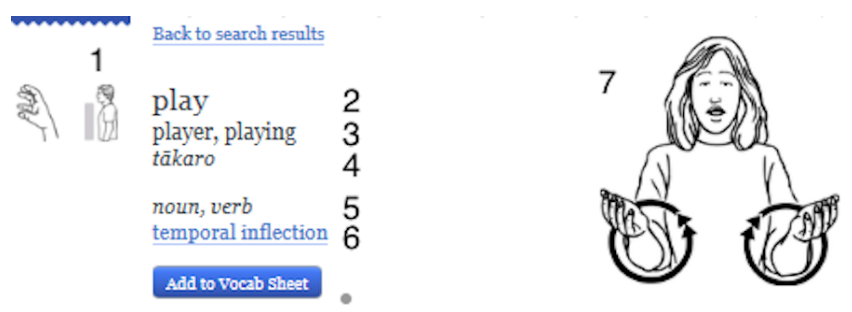

8

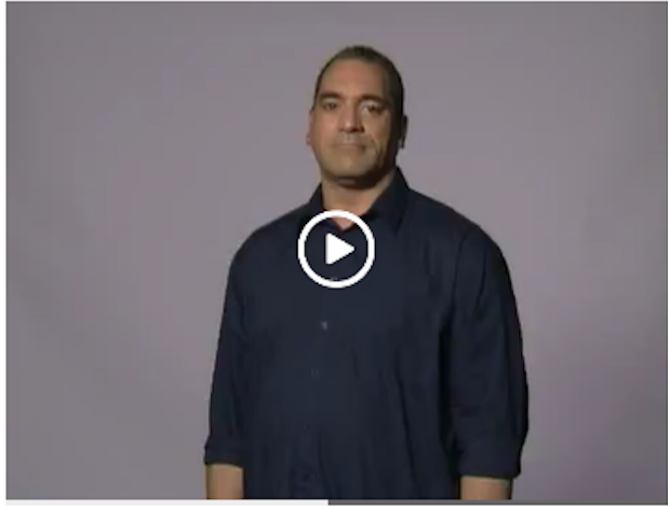

9

Usage Examples

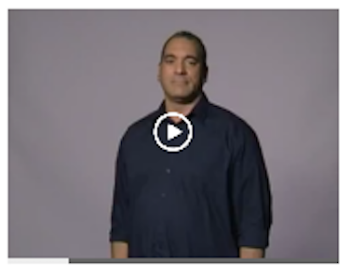

He is very keen on sports and plays socerer and tennis.

he keen sport play socoer tennis he

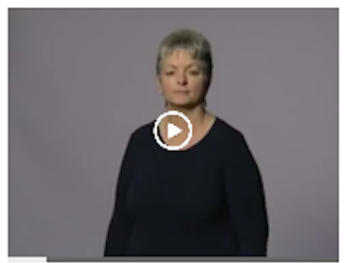

It was raining outside. The two sisters had nothing to do and played with toys inside.

outside rain both sister twiddlethumbs play toy inside

10 Notes Not used for playing a musical instrument.

Figure 4.4: Individual dictionary entry in the ODNZSL

Each entry contains the following elements (numbered in the figure):

1. drawings indexing the handshape and location of the sign;

2. one or more English glosses showing the main sense(s) of the sign;

3. a number of further glosses that are either less common senses or common translational equivalents of the sign;

4. a Te Reo Māori gloss; 
5. word class information;

6. possible inflections, hyperlinked to a glossary in the help menu;

7. a drawing of the sign;

8. a large video showing how the sign is produced;

9. example sentences, consisting of a signed video accompanied by a translation into English, and a glossed representation of the sentence where each gloss is hyperlinked to the relevant entry in the ODNZSL;

10. a usage note and/or a hint for producing the sign where applicable.

Users also have the option to play any video in slow-motion and to add the sign (in the form of the drawing and English and Te Reo Māori glosses) to a vocabulary sheet to be printed or saved as a PDF.

\subsubsection{Participants}

Although an examination of log files does not involve direct interaction with dictionary users, the privacy of these users should be respected. In consideration of this, I decided not to make use of Google Analytics' enhanced 'unique user' profile, that can distinguish between users from the same IP address, and conversely can trace the use of different devices by the same user. The unique user profile also offers detailed demographical data beyond what was required for the limited purposes of this study. If a unique user profile is implemented on a website, it is recommended that visitors are informed that their personal data is gathered from the site and asked for their consent. For dictionary users, this additional step might be a deterrent to using the ODNZSL. Instead, individual users were therefore tracked via 'cookies'. This method still provides an improvement over logging server side requests (where cached pages, for example, cannot be easily tracked); however, inaccuracies may occur due to users blocking or periodically deleting cookies, or being misidentified as unique users when logging in from different devices.

Selection of participants for the Think Aloud Protocol was based on the potential user groups identified in McKee and Pivac Alexander (2008) and also reflects the previously mentioned categorisation by Varantola (2002) of dictionary users as language learners, non-professional users and professional users. 
Participants were recruited through existing networks, both through distribution of an information sheet and through personal invitation to relevant groups, such as networks of sign language interpreters, NZSL classes and the local Deaf community. Twelve volunteers were selected. Table 4.1 shows the selected participants by category and their status in relation to fluency in / use of NZSL.

To establish a more detailed profile of the participants as dictionary users, a short preinterview was held asking about the participants' prior language learning and dictionary use and their familiarity with sign language dictionaries.

\begin{tabular}{|c|c|c|c|c|}
\hline $\begin{array}{l}\text { Varantola (2002) } \\
\text { dictionary user } \\
\text { category }\end{array}$ & NZSL status & $\begin{array}{l}\text { Length of time since } \\
\text { learning NZSL }\end{array}$ & $\begin{array}{l}\text { Amount of time } \\
\text { spent using NZSL }\end{array}$ & $\begin{array}{l}\text { Number of } \\
\text { participants }\end{array}$ \\
\hline \multirow[t]{2}{*}{$\begin{array}{l}\text { Language } \\
\text { learners }\end{array}$} & $\begin{array}{l}\text { Beginner learner } \\
\text { (first year class) }\end{array}$ & $\begin{array}{l}6 \text { weeks of course } \\
\text { learning }\end{array}$ & 4-7 hours a week & 3 \\
\hline & $\begin{array}{l}\text { Intermediate learner } \\
\text { (second year class) }\end{array}$ & $\begin{array}{l}1-2 \text { years of course } \\
\text { learning }\end{array}$ & 4-7 hours a week & 2 \\
\hline \multirow[t]{2}{*}{$\begin{array}{l}\text { Non-professional } \\
\text { users }\end{array}$} & $\begin{array}{l}\text { Hearing friends of a } \\
\text { Deaf person }\end{array}$ & $\begin{array}{l}\text { Minor exposure; no } \\
\text { formal learning }\end{array}$ & Very occasionally & 2 \\
\hline & Deaf community & $\begin{array}{l}1 \text { since early } \\
\text { childhood; } 1 \text { since late } \\
\text { teens }\end{array}$ & Daily (main language) & 2 \\
\hline \multirow[t]{2}{*}{$\begin{array}{l}\text { Professional } \\
\text { users }\end{array}$} & $\begin{array}{l}\text { NZSL tutors / } \\
\text { teachers }\end{array}$ & $\begin{array}{l}\text { Since early childhood } \\
\text { (before age } 3 \text { ) }\end{array}$ & $\begin{array}{l}\text { Daily (main } \\
\text { language ) }\end{array}$ & 1 \\
\hline & NZSL interpreters & $\begin{array}{l}\text { 8-11 years, including } \\
\text { course learning for } \\
\text { 3-4 years }\end{array}$ & Daily (work + social) & 2 \\
\hline
\end{tabular}

Table 4.1: Participants for the interview / Think Aloud Protocol

\subsubsection{Participants' language learning profile}

The groups identified as 'language learners' in table 4.1 were undertaking formal language learning through university classes. Many participants in other categories also had some experience of formal language learning. Many of the students enrolled in an NZSL course were also studying spoken languages, or had done so in the past. Hearing friends of a Deaf person had both formal and informal spoken language learning experiences, and one participant in this category had a home language other than English. The Deaf people in this study considered (written) English to be a second language, and had also learned other spoken / written and signed languages. NZSL interpreters had been involved in extensive 
previous study of NZSL and in one case had also undertaken spoken language courses during their post-compulsory education.

\subsubsection{Participants' dictionary use profile}

All participants with the exception of one owned at least one spoken language dictionary. Eight of the participants owned a monolingual English dictionary (mostly the New Zealand Oxford Dictionary (Deverson \& Kennedy, 2005) and two more had a monolingual English dictionary in the family home as a child although they no longer own one themselves. Although many participants remembered using monolingual dictionaries at school, in adult life they had stopped using them, or only referred to these dictionaries when playing Scrabble, filling in crosswords or playing other language games.

Most participants (7) owned and used bilingual dictionaries for the foreign languages they studied. The majority of these were from L1 -> L2 (5). Three participants owned an L2 -> L1 dictionary. Although several participants owned multiple dictionaries for the same language, nobody had dictionaries in both language directions for the same language pair. Only two participants owned a monolingual dictionary for a language other than English. Other resources mentioned were thesauri, phrasebooks, and specialist crossword dictionaries.

No participants listed online, electronic or pocket dictionaries or phrasebooks. Two participants mentioned that their use of dictionaries had diminished drastically, since using a generic internet search would mostly give them the information they needed.

\subsubsection{Participants' familiarity with sign language dictionaries}

Table 4.2 summarises participants' responses to the questions about sign language dictionaries they were aware of or owned.

Although there is still a high level of awareness of the print dictionaries of NZSL, their use and ownership has mostly been superseded by the ODNZSL and its mobile app version. With the exception of one person (a friend of a Deaf person who had no formal experience of learning NZSL), all participants had used the ODNZSL. 


\begin{tabular}{|c|c|c|}
\hline Name of dictionary & $\begin{array}{l}\text { Know of its } \\
\text { existence }\end{array}$ & Owned / used \\
\hline $\begin{array}{l}\text { Introduction to New Zealand Sign Language } \\
\text { (Levitt, 1986, out of print and no longer } \\
\text { available) }\end{array}$ & 1 & 0 \\
\hline $\begin{array}{l}\text { A Dictionary of NZSL (Kennedy et al., 1997, out of } \\
\text { print) }\end{array}$ & 7 & 3 \\
\hline $\begin{array}{l}\text { A Concise Dictionary of NZSL (Kennedy et al., } \\
\text { 2002, print) }\end{array}$ & 3 & 2 \\
\hline ODNZSL (web) & 11 & $\begin{array}{l}11 \text { (2 occasionally, } 6 \text { regularly, } \\
3 \text { did not specify frequency) }\end{array}$ \\
\hline ODNZSL (mobile app) & 4 & 4 \\
\hline Overseas sign language dictionary (print or video) & 2 & 1 \\
\hline Overseas sign language dictionary (online) & 0 & 0 \\
\hline Other (Total Communication, Gestuno) & 2 & 1 \\
\hline
\end{tabular}

Table 4.2: Participants' use and awareness of sign language dictionaries

\subsubsection{Procedure: Log files}

Data for the ODNZSL was tracked using Google Analytics, a widely available web analytics programme. Standard information tracked by Google Analytics includes the number of visitors, how they arrived on the site, how much time they spent on the site, how many pages they viewed and what site searches they carried out. To track user interaction in more detail, 'Events' were set up to also track:

- the exact search string typed in during a search;

- instances where a user clicked on a video to view it;

- clicks on help items, including the introductory video on how to use the dictionary, the help menu, and links to an explanation of grammatical terms;

- instances where a user clicked on one of the glossed signs in an example sentence;

- the position of a search result of a sign entry when the user clicked on it.

Since these adaptations to the log files were not implemented until March 2014, the selected time period to collect data was the three months of April-June 2014, a representative period which includes the most active months of dictionary use during the year. During this period, a total of 31,753 sessions were logged. The number of users was 16,296 . The number of page views was 319,662, making for an average of 10.07 page views per session. 


\subsubsection{Procedure: Think aloud protocol}

The activity consisted of four parts:

- a short pre-interview;

- a familiarising exercise;

- the Think Aloud Protocol (TAP) exercise;

- a follow-up interview.

The activities took place either in the person's work / study place, or at their home (and in one case at my home).

The need for a rehearsal task prior to a TAP was mentioned by Okuyama and Igarashi (2007). In the current study, participants were asked to imagine they were in a supermarket on their regular grocery shopping trip and to describe their thoughts while walking through the supermarket aisles selecting goods.

For the TAP itself, participants were shown the ODNZSL web page and directed to use the dictionary as they normally would (or if they were not currently dictionary users, to treat this activity as if they were looking up information in a real situation). No specific task instructions were given, but participants were asked to look up at least three items. I remained present in the room during the TAP to deal with technical issues and to prompt participants to 'keep talking' if necessary.

Since some of the participants were Deaf and would be using NZSL during the TAP, several modifications to the procedure were considered. 'Thinking aloud' may not be a feature of sign languages; although there is some evidence for a sign language-based articulatory rehearsal loop equivalent to a 'phonological loop' in spoken languages (Wilson \& Emmorey, 1997), one's own signing is most likely not observed as often, or in the same way, as hearing one's own voice. Also, while navigating through the dictionary a mouse or keyboard has to be used, which inhibits using the hands as articulators at the same time. Since I am a fluent NZSL user myself, I sat opposite the Deaf participant and provided minimal feedback cues (e.g. head nods) to encourage ongoing talk. I made no other comments. Deaf participants were also encouraged to articulate their thoughts before carrying out an action on the keyboard or mouse. Both the screen and the participant were recorded on video. 
The follow-up interview probed further into participants' use of the ODNZSL in this instance and in general. Participants were asked to pinpoint information in the dictionary that they regularly use and information they do not use at all; they were also prompted to explore any problems that they experienced either during the TAP or during their own use of the ODNZSL. Finally, participants were asked to name features that their ideal dictionary would include.

The total length of the interview and TAP varied slightly between participants. The time taken was between 25 mins -45 minutes.

\subsection{Results and discussion}

\subsubsection{Who uses the ODNZSL?}

In line with patterns for other online dictionaries (Johnsen, 2005), the ODNZSL experienced growth in both the number of sessions and the number of users every year since its inception. The proportion of new users continued to rise (see table 4.3), suggesting that while the ODNZSL attracted further interest, in most cases this did not develop into regular dictionary use.

\begin{tabular}{llll}
\hline & Apr-Jun 2012 & Apr-Jun 2013 & Apr-Jun 2014 \\
\hline New users & $8,629(35.9 \%)$ & $11,681(37.2 \%)$ & $14,567(45.9 \%)$ \\
Returning users & $15,390(64.1 \%)$ & $19,690(62.8 \%)$ & $17,186(54.1 \%)$ \\
\hline
\end{tabular}

Table 4.3: New vs. returning users to the ODNZSL

We should bear in mind that the log file data may mistakenly identify returning users as new users because they visit the site from a new device or because they have cleared their 'cookies'. However, the societal factors outlined in section 2.1 - The sociolinguistic context may also have influenced this changing user profile. The 2013 New Zealand Census (Statistics New Zealand, 2013) noted a drop in the number of people who indicated they could have a conversation "about a lot of everyday things" in NZSL (from 24,084 in 2006 to 20,244 in 2013). Some reasons behind this decrease were noted in McKee (2016) and include a lack of support for NZSL in mainstream schools, few opportunities for deaf children to communicate with other deaf peers, funding cuts to adult education programmes that previously offered NZSL classes, and few opportunities for families to learn NZSL. Factors such as these indicate that there may now be fewer learning environments that would support regular dictionary use. Paired with this decrease, however, is a rise in awareness of NZSL by the general public. 
McKee also noted an increase in visibility of a 'Deaf voice' on the internet.

Further support for the dictionary receiving a high level of casual interest but fewer 'serious' dictionary consultations comes from an examination of the frequency and page depth statistics. $45.88 \%$ of visitors were new users and therefore had only visited the website once. A further $22.07 \%$ had visited less than five times, showing that even among visitors logged as 'returning users', there are a large number of casual users. The ODNZSL has a smaller number of highly regular users: $\mathbf{2 . 8 8} \%$ had visited the site more than 200 times, and a further $1.45 \%$ had made between 100-200 visits. Returning users viewed more pages per visit than new users (11.14 vs. 8.81 respectively), indicating that on return visits, users engaged with the website in more depth. $28.2 \%$ of users left the website after only viewing a single page, and new users were more likely to do so. At the other end of the spectrum, $13.46 \%$ of all visits involved viewing 20 or more pages. These in-depth users were more likely to be returning visitors. From the log files, it can be concluded then that although the majority of visitors to the ODNZSL were new users who did not engage with the site in much depth, there was also a sizeable minority of highly regular users who carried out multiple queries each time they visited.

Similar patterns of usage were reported in the interview data. Non-professional dictionary users who were not involved in formal language learning were aware of the ODNZSL's existence but had not used the dictionary beyond an occasional browse out of curiosity. Deaf NZSL users said that they very rarely used the ODNZSL to look up signs or English words for themselves, although in their role as language teachers (both teaching classes and informally 'teaching' friends, colleagues or parents of deaf children) they were frequent dictionary users. In this case, they would look up known signs to add to a vocabulary sheet, but would not look at the entry content in any detail. Responses from beginner and intermediate learners in NZSL classes indicated that they were the most regular dictionary users and looked up several signs daily. The two sign language interpreters in this study (who can be seen both as advanced language learners and as professional users) stated that they only occasionally used the ODNZSL.

\subsubsection{What situations prompt dictionary use?}

Although log files cannot directly reveal users' reasons for using a dictionary, some inferences can be made from examining how they arrived at the dictionary website. The largest source of traffic (65.0\%) was through the use of search engines, mainly Google. Less than a quarter of 
visitors arrived at the dictionary website directly (through typing in its URL or having the page bookmarked). Although it may seem more likely that returning users will be more familiar with the website and will therefore access it directly, in fact they were only slightly more likely to do so than new users (22.68\% vs. 20.82\%). Other traffic showed a sharper contrast, with new users making up the majority of traffic that arrived at the ODNZSL via search engines (11.79\% new vs. 6.98\% returning) or social network sites such as Facebook (6.o6\% new vs. $2.26 \%$ returning).

The search terms that result in a visit to the ODNZSL show that many users may not be looking for the dictionary specifically. 'NZSL dictionary' was only the third most common search term, with the majority of users searching for more generic terms such as 'NZSL' or 'NZ sign language'. Other common search terms were 'learning NZSL', 'basic sign language', 'NZSL alphabet' and various permutations of 'how do you say $\mathrm{x}$ in sign language'.

Reasons participants gave for looking up information during the TAP comprised both communicative and cognitive situations (Tarp, 2009). The TAP did not involve a particular task: participants were left to decide which information to look up. This unguided exercise probably encouraged general browsing of the ODNZSL; many searches were sparked by the participant speaking an English word during the TAP and then wondering how this word was expressed in NZSL; others spotted interesting signs that were not related to their original search in the results and followed through. Nesi (2013 a) noted that electronic dictionaries facilitate browsing through features such as hyperlinks, saving search results and creating vocabulary lists. While the TAP was not an authentic dictionary usage situation, participants also mentioned using the ODNZSL in this way outside of the exercise. An often-mentioned cognitive situation was looking up signs that had previously been learned or seen for rehearsal.

Most of the communicative situations involved language production rather than reception. Participants mentioned wanting to find vocabulary to have a conversation with a Deaf person. For beginners, this involved looking up words or phrases to do with greetings and introductions and themes such as food or family. Both beginner and intermediate learners said they often prepared a conversation topic in advance for classes or when they knew they were going to meet a Deaf person. They wanted to broaden what they could talk about by looking up new signs around a theme, expanding on the standard phrases learned in class. One participant gave the following example: 
so we had a class, and he said 'how are you all?' and I realised I only know how to say 'I'm fine' which is not true! I was like really tired and I was like, it's a complete lie! (laughs) So then I thought actually I should probably learn how to say something else!"

Extending their knowledge of signs learned in class also included looking up grammatical and variation information. One Deaf participant looked up information in the other language direction, i.e. wanting to express a known sign in English. Looking up signs for reception was limited to classroom situations such as translation exercises or watching a video conversation. In real-life situations, participants said they would usually clarify the meaning with the signer on the spot rather than consulting the dictionary.

The authoritative role that dictionaries have traditionally played was also evident. Many users were aware of the relatively high levels of regional and age variation in NZSL and used the dictionary to confirm whether a sign they had observed or had been taught was in common use. A Deaf sign language teacher preferred to choose the particular sign variants in the ODNZSL for inclusion in teaching resources, even when she might use a different variant herself.

Of the 12 participants in the TAP, 8 used both the mobile app and the website. Participants were most likely to use the mobile app in communicative situations when they quickly wanted to look up a sign either for immediate communication or for preparation and rehearsal of vocabulary for classes. The same participants commented that they used the website at home or at work to look up more detailed grammatical information, look at example sentences, and browse the dictionary for interest.

\subsubsection{How is the ODNZSL used?}

\subsubsection{Front and back matter}

The tabs containing front and back matter attracted very few views. The most viewed information was on the fingerspelling alphabet (1.6\% of total page views). All other tabs received less than one percent of page views. The help menu and glossary of terms were accessed least often ( $0.2 \%)$ even though the TAP exercise showed that there was confusion about navigation and about certain usage labels such as 'neologism'.

There were only a few TAP participants who looked at the tabs during the exercise. In the follow-up interview, two participants commented that they had read the information in each tab on first using the dictionary, but did not use it anymore except for the link to learner 
exercises. A beginner participant found the information overwhelming, especially in the 'classifiers' tab.

\subsubsection{Searching}

As mentioned previously, one of the original features of the ODNZSL is its choice of search direction, allowing users to either search by word or by sign features. McKee and Pivac Alexander (2008) found in their survey that $44.5 \%$ of potential users stated they would use all available search options. Log file data show that actual user behaviour is rather different: the overwhelming majority of searches (98\%) were a search by English / Māori word. Searches by sign features only accounted for $0.7 \%$ of all searches, with the remainder being advanced searches (allowing for any combination of sign features, glosses, themes, and grammatical categories). Advanced searches that identified a topic category only constituted $0.2 \%$ of searches. Searches by English / Te Reo Māori word were predominantly for highly frequent words or phrases in English. The top 25 search terms are shown in table 4.4 .

\begin{tabular}{lll}
\hline Search term & Number of searches & Percentage of searches \\
\hline 1. hello & 601 & $0.53 \%$ \\
2. thank you & 498 & $0.44 \%$ \\
3. good & 355 & $0.31 \%$ \\
4. name & 355 & $0.31 \%$ \\
5. you & 344 & $0.31 \%$ \\
6. happy & 341 & $0.30 \%$ \\
7. love & 329 & $0.29 \%$ \\
8. dog & 323 & $0.29 \%$ \\
9 please & 321 & $0.28 \%$ \\
10. play & 315 & $0.28 \%$ \\
11. what & 309 & $0.27 \%$ \\
12. cat & 302 & $0.27 \%$ \\
13. like & 299 & $0.27 \%$ \\
14. my & 296 & $0.26 \%$ \\
15. how are you? & 286 & $0.25 \%$ \\
16. morning & 276 & $0.24 \%$ \\
17. go & 264 & $0.23 \%$ \\
18. I & 260 & $0.23 \%$ \\
19. school & 249 & $0.22 \%$ \\
20. no & 242 & $0.21 \%$ \\
21. want & 241 & $0.21 \%$ \\
22. sorry & 237 & $0.21 \%$ \\
23. friend & 233 & $0.21 \%$ \\
24. and & 230 & $0.20 \%$ \\
25. is & 228 & $0.20 \%$ \\
\hline
\end{tabular}

Table 4.4: Top 25 search terms in the ODNZSL 
The top 25 search terms constituted $6.8 \%$ of all searches in the ODNZSL. This figure is slightly higher if misspellings and phrases containing the same words (e.g. 'my name is') are included. Beginner participants in the TAP looked up similar words and phrases, as did NZSL teachers preparing for a lesson. De Schryver and Joffe (2004) noted that users in their data also mainly looked up very frequent words in the Sesotho sa Leboa-English Dictionary, such as hello / dumela.

Although the log data does not distinguish between English and Te Reo Māori word searches, there were few of the latter, and the most frequently looked up Māori words are considered to be borrowings into the New Zealand English lexicon such as 'kia ora' (a greeting) or 'whānau' (extended family).

Taken together, these findings lend further support to the conclusion that the ODNZSL's main user group is (hearing) people with an interest in learning the language, mostly at a beginner level, who mainly consult the dictionary for language production.

Over the three month period, all 4,300 entries in the ODNZSL were visited or showed up in search results at least once. This coverage demonstrates that the current dictionary content, with its focus on the most frequent signs and words, is in line with the needs of its main user group. However, in total 21,232 different search terms were entered, indicating that there are also unmet needs where either the dictionary content does not include a searched for word, or the search does not identify the target.

\subsubsection{Search results}

In line with the findings of other studies (e.g. Lew, 2004; Lew, Grzelak \& Leszkowicz, 2013), users of the ODNZSL clicked on the first search result more than half of the time (52.82\%), as compared to signs appearing in the second position (20.47\%) and third position (9.89\%). The number of clicks on signs appearing in lower positions steadily declined. It has to be considered that signs are more likely to be displayed in early positions, since all valid searches will have at least one search result but may not have more. The same behaviour can be seen to occur for individual search results, however. For example, the most popular search query, 'hello', returns three different signs. The first search result made up $60 \%$ of the clicks, whereas both the second and third search results were selected $20 \%$ of the time.

This preference for the first search result in the ODNZSL may not signify a lack of sense discrimination on behalf of the user. For example, the most frequently clicked search result 
for 'fine' was the second sign with the sense 'alright, ok' rather than the first sign which has the sense of a monetary fine or punishment.

Interestingly, there is some evidence that dictionary users avoided polysemous signs in favour of signs which have a single sense. An example is that in the search results for the query 'cat', the most frequent sign, which also has the general meaning 'pet', was not selected at all whereas the second search result, with a single gloss 'cat' was selected 147 times. Similarly, a general questioning sign with the sense 'what', 'where', or 'why' was passed over in the search results in favour of a less frequent sign with the single sense 'what'. One participant in the TAP looked up 'nephew' and commented that they would not use the sign which also has the glosses 'niece' or 'son', because “I don't know how specific it is, or how you use it in context”. This suggests that dictionary users consider signs with a single gloss a safe choice, allowing them to avoid ambiguity of meaning when the dictionary information does not contain specific guidance for sense selection.

\subsubsection{Information viewed in the dictionary entry}

Table 4.5 takes as a typical example the entry PLAY to examine use of clickable elements in the entry. With 315 searches, the word 'play' was one of the top 25 search terms in the data. The sign PLAY, the first entry to come up in the search results, was viewed 263 times in total. The elements on the entry page were shown previously in figure 4.4.

As shown in this figure, not all page views involved further interaction with the more indepth information on the page. The most used interactive element was the video of the sign in isolation. The ability to show signs dynamically on video rather than as a static image is hailed as one of the greatest advantages of online sign language dictionaries over printed ones (McKee \& McKee, 2013). In the light of this it is interesting to find that only just over $36 \%$ of page views involved watching the video. This percentage may be somewhat lower than in other cases: the most viewed video for the sign HOW ARE YOU was clicked in $55.84 \%$ of all page views. Overall, the video showing the sign in its citation form was viewed at least once for $93.81 \%$ of all entries, and there were more than 50,000 views in total, showing that this feature is on the whole well used.

One explanation for the low percentage of video views may be the relatively high number of times the sign PLAY was added to a vocabulary sheet. Signs can only be added to a vocabulary sheet from within the individual sign entry and not directly from the search results. In section 4.6.2 - What situations prompt dictionary use?, rehearsal of previously learned signs 
by hearing NZSL learners was mentioned as one motivation for using the ODNZSL, and in section 4.6.1 - Who uses the ODNZSL? it was found that the Deaf participants, when they used the dictionary, often did so in their role as NZSL teachers, whether formally or informally. In both these situations, the immediate purpose of creating a vocabulary sheet would require users to click on the individual sign entry page, but they would not be likely to view the video or other material on the page. An intermediate language learner commented in the interview after the TAP: "It depends. I watch the video if I can't understand the picture from the search." This participant gave an example of looking up the word 'vegetable' during the TAP. As the sign in the search results turned out to have the form of a fingerspelled V-G, the participant did not feel the need to look at the video.

\begin{tabular}{|c|c|c|}
\hline Element & Number of views & $\begin{array}{l}\% \text { of page views } \\
\text { including element }\end{array}$ \\
\hline Total page views & 263 & $100.00 \%$ \\
\hline Video showing sign production & 97 & $36.88 \%$ \\
\hline Slow-motion video showing sign production & 12 & $4.56 \%$ \\
\hline Video example 1 & 9 & $3.42 \%$ \\
\hline Slow-motion video example 1 & 6 & $2.28 \%$ \\
\hline Video example 2 & 15 & $5.70 \%$ \\
\hline Slow-motion video example 2 & 5 & $1.90 \%$ \\
\hline $\begin{array}{l}\text { Grammatical information (link to an explanation of } \\
\text { grammatical terms) }\end{array}$ & 0 & $0.00 \%$ \\
\hline Hyperlinks to other signs in the example sentences & 7 & $2.66 \%$ \\
\hline Add to vocabulary sheet & 79 & $30.04 \%$ \\
\hline
\end{tabular}

Table 4.5: Views of the different elements for the ODNZSL entry PLAY

Example sentences were viewed considerably less often than the citation form video; only $24 \%$ of example videos were viewed. Novice learners in the TAP found the speed and content of the example sentences too challenging, whereas intermediate and advanced learners stated that their user needs had changed over time. For example, one participant said:

"I don't look at the sentences much. I think they were more useful when I was starting, because the sentences are quite simple, so they contain other simple signs, so that can be quite useful when you're first learning. But now, generally you're looking for a specific sign, so you're not as concerned with the other information.” 
Slow-motion views of the videos were viewed regularly; however, this included the videos for signs that are simple to produce such as 'hello' (a wave of the hand). Since 'hello' is also the most popular search term, this suggests that users who were new to the ODNZSL viewed all possible information for this entry, possibly as an operative situation-to find out how to use the features of the dictionary (Tarp, 2009).

Hyperlinks to other content in the dictionary were used least often, although several TAP participants did follow hyperlinks out of general curiosity while they were browsing the dictionary rather than searching for a specific sign.

\subsubsection{Problems and issues}

\subsubsection{Navigation and searching}

During the TAP, participants commented extensively on technical issues such as long loading times and glitches with video playback. If a page was not displayed in seconds, participants would lose patience and click on other parts of the page, try to reload, or give up on the search altogether. This behaviour has implications for the technical design of online dictionaries, especially sign language dictionaries that need to deal with the smooth display of large quantities of videos.

Participants also experienced difficulties because of being unfamiliar with the dictionary interface. A participant who had not used the ODNZSL prior to the TAP spent some time trying to locate the search box and commented in the follow-up interview on the layout of the home page and the need for more prominent search facilities. Other participants missed information because displaying the results required scrolling down. Pagination of search results was also difficult to navigate. It is noteworthy that nearly all participants indicated that the ODNZSL is the first and only online dictionary they have used; in the context of learning other (spoken) languages, they used print dictionaries, and to look up information about English, a general Google search was used instead of consulting an English dictionary (whether in print or online).

Participants' interactions with the ODNZSL interface are coloured by their more general online experiences. Log file data on search terms entered in the ODNZSL showed that users searched for extraneous information, such as song lyrics, names of famous people and other proper names; there were also instances of terms in languages other than the three languages of the ODNZSL. 
The influence of generic web searches on dictionary interface expectations can also be seen in the way search terms were entered as natural language queries. Thus, the data included searches for whole phrases such as 'my name is', 'you owe me chocolate', or 'the bird flew up in the tree', and searches for inflected word forms such as 'am', 'going, 'made', or 'days'. Such queries currently do not return any search results since English glosses in the ODNZSL are not lemmatised.

It was noted in section 3.2 - The format of sign language dictionaries that sign language dictionaries are generally smaller works. The ODNZSL contains just over 4,300 lemmas and mainly covers the most frequently used signs and concepts. It is not surprising, then, that many of the 21,0oo logged search terms did not find a match in the ODNZSL. Data on these failed searches can be used to identify so-called 'lemma lacunae' (Bergenholtz \& Johnsen, 2007). Indeed, since this user study, several of these 'missing' signs, such as TURTLE, PINEAPPLE and SLIDE have been added to the dictionary.

Other search terms that failed to bring up a result may be more difficult to resolve. Firstly, there were searches for auxiliaries, modals and forms of the verb 'to be' that do not have a parallel in NZSL. Secondly, lower frequency English words were searched for, including words from more formal and technical registers (e.g. 'inebriated', 'totalitarian', 'prism'). Participants with intermediate and advanced NZSL skills commented that as they had become more aware of the nature of the NZSL lexicon, they had realised that they needed to modify their search strategies, for example by searching for a higher frequency equivalent such as 'drunk' instead of 'inebriated'. Thirdly, some search terms were words that have only recently entered the English language and may not (yet) have an accepted equivalent in NZSL: e.g. 'Minecraft', 'unfriend', and 'onesie'.

A final problem with inputting a written search query was misspelled or mistyped information. The ODNZSL uses predictive text in the search box to assist with this issue, and some participants acknowledged that this was an advantage of online dictionaries, although in the TAP the correction suggestions were sometimes overlooked.

Section 4.6.3.2 - Searching reported that the alternative search methods provided in the ODNZSL were rarely used. One reason for this may be that the predominant identified user situation was language production by learners of NZSL, requiring a search by word. However, as Schmaling (2012) pointed out, thematic searches are particularly useful for beginner learners and for people who are unfamiliar with using dictionaries. Since beginner learners 
and casual dictionary users were found to be the largest user group of the ODNZSL, a higher rate of searches by topic would be expected. Indeed, search terms such as 'natural disasters', 'personal qualities' and 'zoo animals' were typed into the 'search by word' box, suggesting that users did have a need for thematic categories. Making alternative search facilities more prominent on the website and informing dictionary users how the ODNZSL can be searched would most likely increase the uptake of these features. Some participants in the study mentioned that their NZSL class teacher had demonstrated alternative searches and that they had been given specific class tasks involving alternative searches. This had increased their use of these search methods.

Results from the log files showed that dictionary users treated the 'search by word' box as a generic site search, with some search terms reflecting broader topic categories or information that is found in the front and back matter (e.g. 'fingerspelling chart', 'numbers'). An alternative approach to improving the ODNZSL would therefore be to implement a generic search box that would return results from any of the text in the dictionary. If this approach were taken, attention would need to be given to the ordering of search results to ensure that the most relevant results (i.e. a direct match with a sign entry's gloss) were presented first.

Several participants in the TAP experienced problems when attempting to search by sign features. Beginner learners were not familiar with the handshape and location parameters of signs and stated they did not know where to start. Other participants (including a Deaf NZSL user who tried to use this search method to find an English equivalent for a sign) talked about the difficulties of isolating the specific features of a sign in motion.

\subsubsection{Making sense of search results}

In section 4.6.3.3 - Search results, I discussed the tendency for dictionary users to click on the first search result. This tendency has been noted by many authors alongside other issues to do with dictionary users' lack of reference skills (e.g. Atkins \& Varantola, 1997; Béjoint, 20oo; Tono, 2001). Since the ODNZSL was in many cases the only electronic dictionary that participants in the current study were familiar with, and given that sign language dictionaries display and organise information in their entries differently than dictionaries of spoken languages, it would be expected that users would initially find it difficult to navigate the search results. Beginner users during the TAP did indeed have more difficulties interpreting the search results than participants who had been shown how to use the ODNZSL in classes. 
Other problems affected all participants regardless of their reference skills and language skills. One common difficulty was that some queries returned a very high number of results. This was especially the case after a search by features of the sign, since there are many signs that are produced with a very common handshape such as the flat hand. One participant related an occasion where a search returned 276 results.

Participants also expressed that they found the drawings presented in the search results difficult to interpret when the sign was not known to them. To view the video showing how the sign was produced, participants had to click on each search result in turn and then navigate back to the search results. This led to some confusion and participants commented that they sometimes lost sight of their original purpose for looking up the information during the process of viewing the details in multiple individual entries.

Since beginner language learners were not aware of the complex nature of the sign language lexicon, they were confused by search results that included several alternative signs with the same or similar English glosses. As was discussed in Chapter 3 - Sign language lexicography, sign language dictionaries often include multiple variants in the absence of a standard. However, reliable information on variation is not always available to the dictionary compilers and may therefore not be shown in the entries. In other cases, multiple signs express the same sense reflecting different visual or metaphorical aspects of the activity, emotion or object. For example, figure 4.5 shows the search results for 'soccer', which include iconic depictions of a ball being kicked or bouncing off the head.

\section{Search results for: "soccer"}

3 results

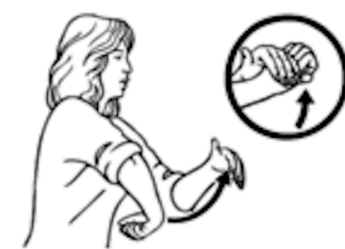

soccer

whana poikiri

football

noun, verb

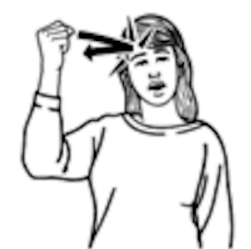

soccer

whana poikiri

football

noun, verb

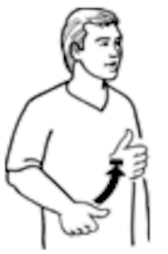

soccer

whana poikiri

football

noun

Figure 4.5: Multiple search results for 'soccer' 
Although search results in the ODNZSL are displayed with the most frequently used sign appearing first, this was not transparent to users. When asked about features their ideal dictionary would have, many participants therefore asked for further information on variation, etymology and frequency. However, when such information was present in the usage notes, participants did not always understand what it meant. For example, one participant did not understand the label 'age variation: older' and wondered if it meant the sign could be produced in different ways to show it was an older sign.

When no further information was available to guide their decision, participants used other clues to guess at which sign would be most appropriate to use. For example, several participants decided to use a particular sign because they recognised the person who demonstrated the sign in the video. Several participants addressed the person in the video directly during their interaction with the dictionary interface, for example greeting the person in the video, saying they recognised them, or even asking them to show the sign again or to slow down. This user behaviour reinforces McKee and McKee's (2013) observation that:

"Video clips are embodied linguistic performance by individuals who have social identities and styles that are immediately recognisable to their own language community, and which will be perceived by learners and other dictionary users as prototypical models of the target language."

The implication of these observed direct interactions is that the format in which information is presented in the ODNZSL may be as influential to users as the information itself.

\subsection{Summary of findings}

The main findings of the two studies reported in this chapter (analysis of log files, and Think Aloud Protocol with brief follow-up interview) were:

Users

- The ODNZSL's largest volume of traffic came from casual, one-off users.

- In the Think Aloud Protocol, beginner and intermediate language learners involved in NZSL classes emerged as the ODNZSL's most frequent and most in-depth users.

- In the Think Aloud Protocol, professionals (Deaf teachers of NZSL and sign language interpreters) indicated that they used the ODNZSL less frequently. 


\section{User situations}

- A general interest in NZSL prompted frequent browsing of the ODNZSL for cognitive purposes.

- The most frequent communicative situations involved language production by (hearing) language learners, either to communicate with Deaf people on a casual basis or in the context of learning NZSL systematically.

- Use of the ODNZSL as an authoritative resource to confirm current language use was also observed.

\section{Usage of the ODNZSL}

- Searches were overwhelmingly in the language direction English—NZSL.

- The most common searches were for highly frequent vocabulary.

- Users avoided polysemous signs or signs with several related translations if an alternative with a single sense or gloss existed.

- The most viewed part of the dictionary entry is the video showing the sign in citation form. However, even this video is not viewed in many cases.

- Front and back matter, including the help menu and grammatical information, are rarely viewed.

\section{Problems and issues}

Unsuccessful dictionary consultations were the result of:

- technical difficulties such as long loading times and missing video links;

- unfamiliarity with the dictionary interface: some users did not know where to input a search, or had difficulties navigating from search results to individual entries and back;

- unfamiliarity with dictionary conventions: users searched for whole phrases and inflected forms rather than citation forms. This type of search was probably influenced by experience with generic internet searches and translation tools;

- the nature of the NZSL lexicon: users searched for English words for which no direct NZSL equivalent exists, either because of structural differences between the lexicons or because the NZSL lexicon has been restricted until very recently; 
- lack of information: due to factors described in section 3.2 - The format of sign language dictionaries, many entries in the ODNZSL do not contain complete grammatical, usage and variation information. The ODNZSL's focus on frequent NZSL vocabulary also means that less frequent NZSL signs, in particular recently developed technical vocabulary, are not found in the dictionary.

\subsection{Limitations}

The main limitations of the present study were:

- the lack of a unique identifier for each user in the log files;

- the lack of available log files for the popular mobile apps;

- the fact that log files could only track events that involved clicking on some part of the dictionary entry.

The inclusion of a Think Aloud Protocol and follow-up interviews partly mitigates against these shortcomings by providing more in-depth observations of users' interactions with the ODNZSL. However, this part of the study only included a very small number of users. The study could have been improved by including further user types, in particular deaf children and their parents and teachers who are tasked with teaching basic NZSL as part of the school curriculum, but who may themselves not be proficient in NZSL. A further improvement would be to increase the number of participants.

Because the research questions for the study were rather broad and there was limited previous data on users of the ODNZSL, the TAP exercise did not specify a particular user situation. Users' lookups during the TAP may therefore not have been typical of their behaviour during authentic user situations.

Recent studies (e.g. Simonsen, 2011; Lew et al., 2013) have used eye tracking successfully as a method for detailed observation of users' interactions with electronic dictionaries. A followup study using this method to track interactions with an online sign language dictionary would be beneficial, especially within the context of a specific user situation such as an NZSL classroom task. 


\subsection{Conclusion}

As a self-contained study, the user study of the ODNZSL described in this chapter has provided an overview of how this online sign language dictionary is currently used. Some of the findings of this study correspond with user responses to previous surveys (Moskovitz, 1994; McKee \& Pivac Alexander, 2008) prior to the development of the ODNZSL. In particular, the current study confirmed that one of the dictionary's main functions is as a tool for language learning; that the main user groups are hearing language learners; and that their user need is predominantly for language production. In other respects, objective data from observed user interactions showed that some features of the dictionary are used much less often in practice than subjective survey responses would predict. This was the case with videos showing examples of a sign used in context, drawings, and searching by features of a sign.

The current study has also highlighted problems that users of the ODNZSL experience. Data on unsuccessful dictionary lookups can help to improve the ODNZSL and other sign language dictionaries. Firstly, this data helps to identify 'lemma lacunae' (gaps in the content of the dictionary). Secondly, improvements to general navigation could be made, although any changes to scrolling, pagination of search results, and video display need to be weighed up against possible increased page loading times. Thirdly, search methods could be adjusted in acknowledgment of the changing behaviour of dictionary users in the digital age that was also noted by Lew and de Schryver (2014). Users expect to be able to enter natural language queries and inflected forms, for example. Adding lemmatisation of the English glosses in the ODNZSL and allowing searches for other fields (such as topics or grammar information) within the same search box may improve the 'hit' rate of search results. Fourthly, one way to balance conflicting user needs would be to explore customising how the dictionary content is displayed for different users. By displaying the most looked for information early on in the search results (for example, by allowing users to play the main sign video directly from the search results without needing to click through), beginner language learners can be shown the essential information in a way that keeps the information cost low. More advanced users can then click through to more detailed information. Lastly, the findings of this study suggest that providing training for users to become familiar with the features of the ODNZSL is an important step to increase successful lookups.

While suggested general improvements such as these do not address the main thesis question directly, the effectiveness of including signed definitions is ultimately affected by the manner 
in which these definitions are presented, and whether dictionary users will be able to locate and make sense of this new information. The findings of this study therefore not only make an indirect contribution to the thesis as a whole, but they have wider implications for the ODNZSL and other online sign language dictionaries. The study also contributes more broadly to our knowledge about dictionary users in the digital age and how they interact with novel dictionary formats and features.

For the purpose of the thesis, the study aimed more specifically to determine whether there was a user need for (signed) definitions in sign language dictionaries. A user need was defined as a need for information experienced by a particular type of user in a specific situation (Tarp, 2009). Definitions, as information about word meaning, may serve different purposes depending on the kind of user and the kind of situation. At least four distinct user needs can be identified in relation to definitions.

1. As was discussed in section 3.4 - Dictionary typology: some distinctions, definitions have typically been a central element in monolingual, general descriptive dictionaries. In this context definitions are intended to meet the receptive, or decoding, needs of native language users in both communicative and cognitive situations, particularly for infrequent or rare vocabulary, since native language users are likely to already know the meaning of more frequent words (e.g. Summers, 1988; Béjoint, 200o).

2. In dictionaries that address language for specific purposes (LSP), such as technical dictionaries, both linguistic and encyclopaedic knowledge about the meaning of the lemma may be required (Kwary, 2011).

3. Definitions have also been included in monolingual learners' dictionaries and in 'bilingualised' dictionaries, which combine definitions in the target language with translational source language equivalents (Laufer \& Melamed, 1994). In these dictionaries, definitions are targeted at language learners and potentially help these learners with both receptive / decoding and productive / encoding needs. Depending on the level of the language learner, they may have need of definitions of high frequency or lower frequency vocabulary for sense discrimination in communicative situations.

4. Definitions may also play a role in developing vocabulary knowledge beyond the immediate communicative situation, although their effectiveness at contributing to vocabulary learning has been disputed (e.g. Nation, 2001) and may depend on the quality of the definition (e.g. McKeown, 1993; Nist \& Olejnik, 1995). 
Evaluating these user needs in the light of the findings of this study is necessarily limited to the context of existing multifunctional, bilingual sign language dictionaries such as the ODNZSL. With regard to the first user need, for example, there may well be an unrecognised need for Deaf NZSL users to look up the meaning of infrequent signs in monolingual resources. However, until such resources exist, this user need cannot be evaluated objectively. In the present user study, Deaf NZSL users were unlikely to use the ODNZSL to look up the meaning of unknown signs. Looking up signs for decoding also requires these signs to be locatable without searching by an English equivalent. The search by sign features, when attempted, proved to be problematic even to native NZSL users.

The second user need was found to be present; however, the ODNZSL is not likely to be able to meet this user need as it is primarily a general purpose dictionary with a focus on frequent vocabulary. Both interpreters and intermediate users were found to look up technical vocabulary related to their work situations or area of study, but commented that they were often unsuccessful in finding these signs in the ODNZSL. As was mentioned in section 3.4.4General vs. restricted, signed definitions have indeed appeared in technical sign language dictionaries such as the British Sign Language Glossaries of Curriculum Terms (Scottish Sensory Centre, n.d.).

Learners of NZSL, especially those involved in formal NZSL learning through classes, were found to be regular users of the ODNZSL. NZSL learners used the dictionary both in communicative situations and as an aid to vocabulary learning. The third and fourth user needs are therefore the most suitable focus for the following chapter and for the final evaluation of the usefulness of signed folk definitions. In particular, the user needs of intermediate NZSL learners will be considered. This user group's predominant need is to acquire mid- and low-frequency vocabulary (Humblé, 2001) which is more likely to require additional information for sense recognition and word / sign knowledge. Intermediate NZSL learners are also more likely to be able to make use of information presented in NZSL as the target language, whereas beginner learners may not have sufficient fluency to attempt to view longer stretches of signed material.

In the next chapter I will discuss the role of definitions for language learners in more detail. I will describe a test carried out with intermediate NZSL learners to determine for which types of lexical item these users have a need for further information. The question whether signed definitions can effectively meet these user needs will be taken up in later chapters. 


\section{Chapter 5: Evaluating the user need for definitions}

\section{Introduction}

In the first chapters of this thesis, I concluded that the possible contributions of signed folk definitions need to be evaluated in the context of existing multifunctional, bilingual sign language dictionaries. The Online Dictionary of NZSL (ODNZSL) is an example of such a dictionary, with both descriptive and pedagogical aims. Although definitions can potentially contribute to both these aims, indiscriminately creating a definition for all existing dictionary entries may lead to an undesirable situation where "dictionary compilers make great efforts to define words that no user ever looks up." (Béjoint, 200o, p. 97). A more practical approach for lesser-resourced sign languages is therefore to first target a particular user group and user situation, and then to identify the lexical items for which these users are most likely to have need for a definition.

The previous chapter outlined a user study of the ODNZSL that identified adult, hearing, intermediate learners of NZSL as the most likely group to require and make use of signed definitions, especially for the purposes of language production and vocabulary learning.

In the current chapter I will focus more closely on the user needs of this target group. I will first give an overview of the literature regarding the use of dictionaries in language learning, and the role definitions play in this context. This overview will describe the potential functions of dictionaries in decoding, encoding, and vocabulary learning. There is some uncertainty about the role and effectiveness of definitions for these purposes. I will therefore examine other elements of the dictionary entry that are relevant to the same user needs. Firstly, I will discuss reasons that equivalents in bilingual dictionaries (including sign language dictionaries) are not a satisfactory substitute for definitions. Secondly, I will examine how examples of use contribute to language learners' understanding and production of the target language. In common with other dictionary elements, examples will be found to be most effective when they are targeted at particular user needs. I will briefly discuss the multiple functions of examples in the ODNZSL to show that these examples do not currently meet learners' user needs in all cases.

Having identified particular categories of lexical items for which current dictionary information may be inadequate, I will test which of these categories are most problematic for 
intermediate sign language learners. I will describe how I developed an online 50-item test with sections for comprehension and production based on an error correction format, and how this test was administered to two small groups of intermediate sign language learners involved in university courses. The results of the test did not identify any category as being more problematic than others. I will therefore conclude that a small selection of signs from different categories should be used to elicit folk definitions.

\subsection{Dictionaries and language learning}

\subsubsection{Purposes of dictionary use}

In the previous chapter (Chapter 4 - A user study of an online sign language dictionary), reference was made to three different purposes that language learners have in using dictionaries:

- decoding: finding out the meaning of a lexical item, within the context of coming across an unfamiliar lexical item while reading, or more rarely listening to, a text in the target language;

- encoding: finding out how a lexical item is used in the target language, with the purpose of using it in a written, or more rarely spoken, target language text;

- vocabulary learning: finding out about both the meaning and use of a lexical item to add it to one's target language lexicon.

The first two of these purposes are communicative whereas the third purpose is cognitive (Tarp, 2009). In communicative contexts, learners have an immediate need to apply the information they find in the dictionary. Often this will be in the context of a classroom task such as reading, writing, or a translation activity. The priority will therefore be to locate the information most relevant to the task quickly in the dictionary. In cognitive situations, the dictionary potentially provides in-depth information on form, meaning and use that contributes to word knowledge (Nation, 2001). Since this information is not immediately applied, the speed of the lookup is not as crucial. The intelligibility of this information is the most important factor in this case.

As dictionary users, language learners differ from native language users in several ways. Firstly, while first language (L1) users also use dictionaries for decoding and for cognitive purposes, they mostly look up unusual, infrequent or rare lexical items (e.g. Summers, 1988; Béjoint, 200o), whereas second language (L2) learners need to find out the meaning of highly 
frequent vocabulary to understand target language texts. Secondly, as language learners progress, their need for encoding information becomes the dominant purpose for using a dictionary (Humblé, 2001). Native language users may only need to know how to spell a word in order to produce it, but language learners require much more information including spelling, pronunciation, inflections, grammatical structure, collocations and cultural restrictions (Jackson, 2002). Finally, language learners are likely to use dictionaries as an explicit vocabulary learning strategy.

Nesi (2013 b) summarised a number of older studies that found a preference for dictionary use during decoding activities and especially for activities involving the written rather than spoken medium. Dictionary use for spoken language is mainly limited to preparation for controlled class activities, since it is not feasible to carry out a dictionary query during a normal spoken interaction (Rundell, 1999). The activities involved in learning a sign language differ from this pattern since sign languages are largely unwritten languages (see section 3.2.1 - Representing signs without a written form). Instead of reading and writing, learners of a sign language can therefore be expected to spend most of their time on class and independent learning activities involving face-to-face signed interaction and video texts. In the user study of the ODNZSL (see section 4.6.2 - What situations prompt dictionary use?), learners at all levels of proficiency were found to be far more likely to use the ODNZSL for encoding activities. This is in contrast to Nesi's (2013 b) finding that generally, beginner learners use dictionaries more when reading (a decoding activity), while advanced learners used dictionaries more when writing (an encoding activity). In other respects, however, the purposes for which sign language learners use dictionaries correspond with those of learners of spoken languages and the user needs for learners in general are likely to be more similar than the user needs of native speakers or signers.

\subsubsection{The choice of dictionary formats}

To understand the role definitions play in meeting the different purposes of language learners, I will first return briefly to the distinction between monolingual and bilingual dictionaries made in section 3.4 - Dictionary typology: some distinctions. In a monolingual dictionary, the definition forms the central part of the entry, whereas bilingual dictionaries pair lexical units in one language with a series of equivalents in another language. Both monolingual and bilingual dictionary entries may contain further information about the headword's form, meaning and use, including example phrases or sentences, and grammar, register and variation information. Monolingual dictionaries generally include more 
extensive information and more lemmas than bilingual dictionaries, although this will depend on the purpose and target group of the dictionary.

The choice of dictionary is determined not only by the relevance of different information to the purpose of the learner, but also because this information needs to be located first. Research into the effectiveness of these different formats has shown a general preference for bilingual dictionaries. In a large-scale survey and test carried out by Atkins and Knowles (1990), 75\% of students chose to use a bilingual dictionary. Bilingual dictionaries may be especially suited to communicative functions because they provide a reasonably quick answer to the user's query in the immediate lookup situation, although Luppescu and Day (1993) found that even using a bilingual dictionary drastically increased the time spent on reading a text.

However, Atkins and Varantola (1997) found that the choice of dictionary format was determined by a more complex set of four factors:

- the language direction of the task the learners were involved in;

- the kind of information searched for;

- the proficiency level of the learners;

- the previous searches for information about a word in other dictionaries.

\subsubsection{Dictionaries and vocabulary learning}

As a deliberate focusing strategy, using dictionaries can assist vocabulary learning, according to Nation $(1989 ; 2001)$. Although the overall impact of a single dictionary lookup on learning vocabulary is very small, Nation argued that dictionaries made a valuable contribution when combined with other vocabulary learning strategies. and that this contribution could be enhanced by reconsidering the type and format of information in learners' dictionaries.

Nation (2001) generally supported the use of bilingual dictionaries, stating that the process of linking new words in the foreign language with known vocabulary in the learner's first language is an effective way of reinforcing vocabulary learning. However, he argued that bilingual dictionaries could be seen as "a complement, rather than a competitor, to monolingual dictionaries" (p. 290).

With regard to monolingual definitions, Nation (1989) argued that it was more useful to present words in sentence contexts rather than in a formal, context-free definition, and that 
broad definitions covering as many different uses of a word as possible were required rather than exhaustive definitions for separate word senses. Nation also noted that definitions that explain the meaning and relationship of individual productive word parts would help language learners to understand other vocabulary with these word parts more quickly.

Nation (2001) also reviewed the role of spoken definitions in language learning. This discussion will be relevant in the next chapter (Chapter 6 - Folk definitions) since the proposed format of signed definitions has much in common with the kind of definitions provided by teachers during classroom discourse.

\subsubsection{Dictionaries and decoding}

Decoding tasks involve finding out the meaning of a word from the language learner's L2. Learners can look up the word in either a monolingual dictionary or in the part of a bilingual dictionary that lists L2 headwords with equivalents in the learner's L1. For decoding, the usefulness of definitions can therefore be compared with the usefulness of Li equivalents.

In Atkins and Varantola's (1997) study, learners initially overwhelmingly chose bilingual dictionaries, and this preference was strongest for beginner learners. These learners may not have sufficiently developed skills in their L2 to understand the definition and other material in a monolingual dictionary (see also Carter \& McCarthy, 1988). The Li equivalents in the bilingual dictionary will generally be more easy to understand. A significant disadvantage, however, is that equivalents not only provide less information than definitions, but that in some cases, equivalents may mislead users as to the meaning of the target word (Luppescu \& Day, 1993). The issue of lack of equivalence in bilingual dictionaries will be discussed further in section 5.2 - Equivalents or glosses.

Atkins and Varantola's study confirmed that learners were aware of the disadvantages of L1 equivalents. As their proficiency in their L2 increased, learners began to use monolingual dictionaries more (see also Carter \& McCarthy, 1988; Atkins \& Knowles, 1990). In particular, Atkins and Varantola noted that a lookup in a monolingual dictionary often followed several unsuccessful lookups in a bilingual dictionary. However, they also found that although advanced learners were more likely to use monolingual dictionaries, they were less likely to be confident that they had located the information they needed.

For languages with a large target market of learners (particularly English), monolingual learner's dictionaries have been developed such as the Longman Dictionary of Contemporary 
English (Summers, 1987), the Collins COBUILD English Language Dictionary (Sinclair, 1987 a), the Oxford Advanced Learner's Dictionary of Current English (Hornby \& Cowie, 1989) and the MacMillan English Dictionary for Advanced Learners (Rundell \& Fox, 2002). These dictionaries focus on more frequent vocabulary and provide guidance notes or signposts that are intended to improve the success rate of the lookup and to help learners locate the required information more quickly. Definitions in learner's dictionaries are written so that they can be more easily understood by learners with lower proficiency levels: they may use a controlled vocabulary (e.g. Carter \& McCarthy, 1988) or a full sentence format based on everyday informal explanatory strategies (Hanks, 1987). The effectiveness of different definition formats has been a frequent subject of discussion (e.g. McKeown, 1993; Nist \& Olejnik, 1995; Rundell, 2006). The suitability of different definition formats for sign language dictionaries will be discussed in more detail in Chapter 6 - Folk definitions.

\subsubsection{Dictionaries and encoding}

In encoding tasks, learners know the meaning they are trying to express and need to find out how this meaning is expressed in the L2. A bilingual dictionary (L1-L2) fits this task well, especially when the L2 equivalents are accompanied by examples of use. However, the more extensive information in monolingual dictionaries may still be required especially by advanced learners. Definitions can provide information to help encoding learners distinguish between different senses of the L2 word. Definitions in full sentence format also show typical patterns of use (Hanks, 1987).

While definitions are potentially of value for encoding tasks, in a monolingual dictionary this information is not easily locatable since learners do not know the form of the target word to look up. In Atkins and Varantola's (1997) study, translators and other advanced language learners therefore initially looked up possible L2 equivalents in a bilingual dictionary and then consulted one or more monolingual dictionaries to find out more information about each of the equivalents. To shorten this laborious process, a new format of dictionary has been developed. In so-called 'bilingualised' dictionaries, entries from a monolingual (often a learner's) dictionary, including a definition, are supplemented with translational equivalents in the learners' Li. This format potentially suits a broad cross-section of learners at different levels (Laufer \& Melamed, 1994; Laufer \& Hadar, 1997; Laufer \& Kimmel, 1997).

Some studies found that individual learners may not use all of the information in bilingualised dictionary entries as intended. For example, Laufer and Kimmel (1997) found 
that most lookups in their study of a bilingualised English-Hebrew dictionary only involved learners accessing the information in either Hebrew or English. Similarly, Thumb (2004) found in her think-aloud study that most learners preferred to look up either the L1 translational equivalents or the L2 definition, but not both, and that they mainly stuck to strategies they were familiar with. However, in Laufer and Kimmel's study many learners did make use of the information in both languages, but not simultaneously and not for all entries. Humblé $(1998,2001)$ argued that the need of encoding learners for specific information on meaning and use is partly predictable from the lemma's frequency level. These findings suggest that definitions are likely to be most effective when they are targeted at particular lexical items.

\subsubsection{Relevance to the thesis}

With regard to the present thesis, the findings in support of combining the features of monolingual and bilingual dictionary formats are relevant because the addition of signed definitions would result in a similar hybrid format. The current bilingual structure of sign language dictionaries, including equivalents in the spoken L1, is helpful for encoding and will especially suit beginner learners; however, as was shown in this chapter, learners with higher proficiency levels would benefit from L2 definitions for sense discrimination, and signed definitions could also provide information on typical patterns of use that are essential for encoding. L2 definitions provide more in-depth sense information than equivalents and therefore support decoding learners, provided that the definitions are intelligible. For vocabulary learning, although the link to learners' first language made by the spoken L1 equivalents is helpful, signed definitions that cover a broad set of uses of a sign and that draw learners' attention to the individually meaningful parts of the sign may enhance vocabulary learning. This may especially apply to polycomponential signs (see section 2.2.4.1 - Depicting signs or polycomponential signs) or signs which are based on a visual metaphor (see section 2.2.3.4 - Metonymy and metaphor).

The main difference between the proposed hybrid format of sign language dictionaries and existing bilingualised dictionaries is that the latter are founded on comprehensive monolingual data. In bilingualised dictionaries, all lemmas are already defined and the main lexicographical task is to add translational equivalents and examples to help learners with encoding. Sign language dictionaries do not have the same level of monolingual data available. The findings of Laufer and Kimmel (1997) and Humblé (2001) that definitions are not equally accessed for all lexical items support a selective approach for sign language 
dictionaries, where definitions are only provided in cases where the current information does not adequately meet the needs of dictionary users.

The aim of the remainder of this chapter is to identify representative sign lemmas in the ODNZSL for which definitions would be beneficial for the target group of hearing, adult, intermediate L2 learners of NZSL. With this aim in mind, I will first examine the role of two main existing elements of bilingual sign language dictionary entries: equivalents or glosses, and examples of use.

\subsection{Equivalents or glosses}

\subsubsection{Degrees of equivalence}

The basic assumption underlying the format of bilingual dictionaries is that two words in different languages can be equivalent in both meaning and use. This assumption is even more likely to be made by users of sign language dictionaries, firstly because, as was mentioned in section 3.2.1 - Representing signs without a written form, the signs themselves have no independent written representation, and secondly because of the still widespread perception that signs either depict real-life actions and objects in a transparent way, or that they are visual representations of spoken words.

In fact, due to the inherent anisomorphism between languages described by Zgusta (1971), complete equivalence is very rare. It is more common in bilingual dictionaries to find situations of partial equivalence, where a target language lexical item overlaps semantically with the headword but is not identical, with differences not only in semantics but also in grammar or usage. There may also be situations of zero equivalence (e.g. Adamska-Salaciak, 2013), where no equivalent lexical unit exists in the target language for a dictionary headword.

\subsubsection{Types of equivalence}

Different relationships exist between the dictionary lemma and its equivalents in the target language, or, more strictly in the opinion of some authors (e.g. Atkins \& Rundell, 2008) between lexical units in the source and the target language. Zgusta (1979 / 2006; 1987) distinguished between translational equivalents and explanatory equivalents. Translational equivalents are 'insertable' in a target text and provide a smooth translation for the headword in a particular context. Explanatory equivalents, on the other hand, paraphrase the meaning of the headword (or a particular sense of the headword), but are not themselves necessarily lexical units in the target language. 
Adamska-Salaciak (2010) reviewed various other distinctions and proposed an expanded model of four different equivalence relationships:

- cognitive equivalence - a target language lexical unit that covers the general, decontextualised, prototypical sense of the headword;

- explanatory equivalence-a descriptive paraphrase of the headword's meaning;

- translational equivalence - a lexical unit that translates the headword in a specific context;

- functional equivalence-a target language expression that fulfils the same function as the headword in a specific context.

The first two types of equivalence are relatively decontextualised, whereas the last two types are context-dependent. Cognitive and translational equivalents are lexical units in the target language that may share not only semantic characteristics with the source language lexical unit, but also grammatical and usage characteristics; explanatory and functional equivalents are paraphrases or expressions that are unrelated to the grammatical form of the headword.

The significance of these various equivalence relationships is that each kind of equivalent meets certain dictionary user needs, but may lead users to experience problems in other areas. For example, while an explanatory equivalent provides information about the source language lemma that will help language learners with text comprehension, when the same explanatory equivalent is used in language production, the user will sound unidiomatic. Zgusta (1971, p. 320) gave an example of the French equivalent 'état de fille' for the English word 'girlhood': while this equivalent correctly shows that the English word refers to female young people only, in practice French speakers would use a gender-neutral expression such as 'adolescence'. A series of translational equivalents on the other hand may serve language production purposes better, but may leave users uncertain about the prototypical meaning of the headword or about which sense(s) a particular translational equivalent covers.

\subsubsection{Types of equivalence in the ODNZSL}

The purpose and intended user group of the dictionary, then, determine the usefulness of a particular type of equivalent. For this reason, the two halves of a bilingual dictionary are usually asymmetrical: i.e., the equivalents presented when the language is the source language are different when the same language is the target language. In multifunctional, bidirectional online sign language dictionaries, however, there are no separate sections for the two language directions. The same dictionary entry is displayed regardless of whether the user searched by a spoken language word or by features of a sign. In this context, glosses take 
on a dual role. They act at the same time as headwords for a search by a spoken language word for a sign, and as target language equivalents describing the meaning of a (source language) sign.

The problems arising from this dual function were already mentioned in section 3.4.1 Monolingual vs. bilingual, where it was also stated that providing multiple glosses partly mitigates against users equating the gloss with the meaning of the sign. References to 'main' and 'secondary' glosses in McKee and McKee's (2013) discussion of the creation of the ODNZSL indicate that the main gloss(es) were primarily intended to capture the prototypical sense(s) of the sign lemma, i.e. as cognitive or explanatory equivalents. Secondary glosses were conceived as additional translational or functional equivalents. An example of this intended distinction between main and secondary glosses can be found in the following description and the corresponding dictionary entry in the ODNZSL (figure 5.1).

“... the sign glossed as BUILD may also translate in context as 'builder, carpenter, construct, nail'..." [emphasis added]

(McKee \& McKee, 2013, p. 520)

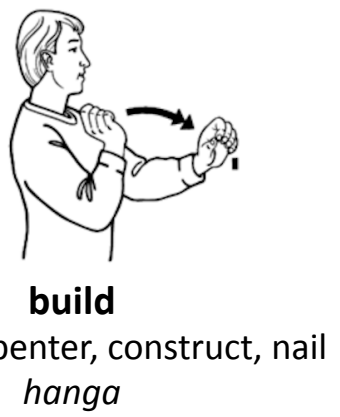

Figure 5.1: Main and secondary glosses for the ODNZSL entry BUILD

\subsubsection{Categories of non-equivalence}

Zgusta (1971) argued that "anisomorphism must be expected in all lexical units and can be found in most of them." (p. 296). While differences between lexical units can be found in nearly all domains, a number of lexicographers (Zgusta, 1971; Snell-Hornby, 1990; Duval, 2008; Atkins \& Rundell, 2008) have addressed the question whether there is more divergence in some lexical categories than in others. I will first summarise the conclusions of these authors and then I will illustrate how they apply to entries in the ODNZSL.

Zgusta (1971) and Duval (2008) identified both extralinguistic and linguistic differences between lexical units. Extralinguistic differences (labelled by Duval as differences in the signified) arise when referents in the real world exist in one culture but not in the other. Linguistic differences (or differences in the signifier) occur when one language labels a 
referent but another language doesn't at all, or in a different way. Snell-Hornby (1990) identified specialist terminology / nomenclature and internationally known items and sets as the categories most likely to have equivalent semantic content and therefore to be least problematic for language learners. Zgusta, however, pointed out that non-standard (minority) languages may have onomasiological gaps in these areas where many terms have not yet stabilised in use. When these languages are the target language in a bilingual dictionary, it may be difficult to find equivalents even when the referent exists in the culture.

At the other end of the spectrum, when unique referents exist in a particular culture, other languages may not have an equivalent word to refer to it. Zgusta gave examples of culturebound terms for flora and fauna unique to a particular region as well as objects in the material culture (such as types of shop), while Duval also included abstract referents such as political bodies and significant dates.

Other differences in semantic content arise when two lexical items denote the same referent but have different connotations (Zgusta, 1971; Duval, 2008; Atkins \& Rundell, 2008). SnellHornby (1990) found that this type of non-equivalence was particularly likely in words expressing perception and evaluation, often linked to sociocultural norms. This category of words is not necessarily problematic for the purpose of decoding, but may cause difficulties for encoding language learners. For this reason, Duval stated that an equivalent may be found that captures the same connotation even though it denotes a different referent. Duval gave the example of using the English translational equivalent 'snakes and ladders' for the French 'jeu de l'oie' (literally 'game of the goose'), since the connotation of a children's board game where a player's token advances along a set of squares may be more important than the actual game.

Duval stated that further linguistic differences arise when lexical items differ in their extension-i.e. the lexical unit in one language has a similar sense but refers to a wider or narrower range of referents than its equivalent in the other language-or their comprehension (the features specific to that concept). Atkins and Rundell's example of the relationship between the English 'teacher' and the French 'professeur' falls in this category, and it is also likely to affect Snell-Hornby's category of 'concrete objects, basic activities, and stative adjectives'.

Moving on from differences in semantic content, Atkins and Rundell (2008) distinguished four further relevant areas: 
- collocational context;

- vocabulary type (e.g. differences in register, style, region, or attitude);

- message: this category includes idioms, proverbs and sayings for which an equivalent may have the same pragmatic force but not the same literal meaning;

- function: this comprises semantically 'empty' grammatical words or function words.

These four categories especially pose problems for encoding language learners. Zgusta (1971) emphasised function words and interjections as being particularly difficult, whereas Duval (2008) focused on the treatment of proverbs and idioms in bilingual dictionaries.

\subsubsection{Non-equivalence in the ODNZSL}

\subsubsection{Semantic content}

\section{Onomasiological gaps}

In spite of rapid expansion of NZSL vocabulary in recent years, there are still onomasiological gaps in many domains. In section 4.6.4.1 - Navigation and searching, users of the ODNZSL were found to look up many English words that did not have an equivalent NZSL sign. While such gaps are problematic for sign language learners, they will not be further discussed here since the purpose of this chapter is to identify existing signs that would benefit from being defined.

Gaps in the other language direction, i.e. signs that have no equivalents in English, also exist although they are less noticeable to dictionary users, because all signs included in the ODNZSL are accompanied by glosses of some kind to enable bidirectional searching. The problems that the dual role of glosses potentially cause for users were mentioned in section 5.2.3 - Types of equivalence in the ODNZSL.

It is interesting to note that there are entries for which no equivalent in Te Reo Mãori has been included. Some of these entries describe referents that would not have been present in Māori culture and would therefore not have been named: for example, MEERKAT, GRAPEFRUIT, or LASAGNA. The grammatical use of the NZSL sign EXIST also did not have a suitable equivalent in Te Reo Māori. Finally, some of these entries were culture-bound signs; these will be discussed in the next section. 


\section{Culture-bound signs}

As Stokoe et al. (1965, p. xxvii) pointed out, Deaf sign language users form a part of the wider regional or national culture surrounding them. Many of the objects, artefacts and institutions of this culture, for example kinds of food, clothing, buildings, kinds of transport etc., will therefore be shared between Deaf and hearing people. However, Deaf community members also share experiences, beliefs, attitudes, history, norms, values and artistic expressions with other Deaf people that are different from the experiences they share with hearing people on the basis of a shared national or local identity. Although the term 'Deaf culture' is still contested and there is a lack of research on the features of such a culture (Ladd, 2003), we could therefore consider certain signs to be 'culture-bound' since they have no direct equivalent in the surrounding spoken language; or when an equivalent term exists, the spoken / written word has very different connotations and frequency of use.

Examples of culture-bound signs in the ODNZSL include a few objects from the material culture that are used by people with a hearing loss, such as flashing doorbells, vibrating alarm clocks and TTYs (an early telephone that converted typed text into signals that could be transmitted through an ordinary telephone; now almost obsolete due to the advent of mobile phones and SMS messaging). There are also institutions and organisations that are frequently referred to within the New Zealand Deaf community such as Kelston and Van Asch/ Sumner Schools for the Deaf, and Deaf Aotearoa, the national member organisation (and service provider) of Deaf people in New Zealand. Although no direct equivalents for such signs exist in the spoken language, they can usually be described by explanatory glosses and usage notes providing relevant (encyclopaedic) information.

More complex from a lexicographical point of view are those signs that describe people in terms of their identity or relationship with the Deaf community. Three examples of such signs are shown in figure 5.2. The English equivalents for these signs only describe whether, and to what degree, a person's sense of hearing is functioning, whereas the signs also identify people's status as insiders or outsiders in the community and therefore generate associated 'frames' (Fillmore, 2003) about the language use, behaviour and experiences of the people being described.

While these signs are likely to be amongst the first to be taught in sign language classes, intermediate learners may still have some difficulties in understanding their full semantic content and use. Culture-bound signs such as these will therefore be included in the test that I will report on later in this chapter. Regardless of whether culture-bound signs prove to be 
problematic for learners, their key role in conveying core cultural information and the frequency with which they are used in sign language discourse means that they are good candidates for definitions. The only non-technical dictionary (to my knowledge) that has included signed definitions, the BSL CD-ROM Dictionary of Deaf Community and Culture (Brien et al., 1997), focused exclusively on this category of signs.

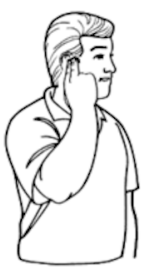

Deaf

turi

deaf, deaf person, deafness

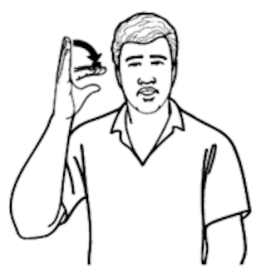

go deaf, hearing impaired

turi, turi haere

deaf, deafened, hard of hearing,

lose hearing, partially deaf

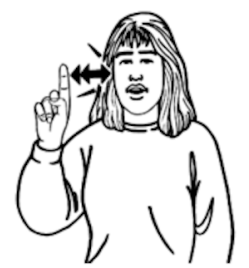

hearing

rongo

Figure 5.2: Signs referring to people in terms of their hearing status and relationship to the Deaf community

\section{Extension and comprehension}

One type of sign that may show differences in extension and comprehension is polycomponential signs (see section 2.2.4.1 - Depicting signs or polycomponential signs). For example, the sign shown in figure 5.3 includes a handshape that is used as part of the productive lexicon of NZSL (and other sign languages) to denote small, round objects. In the partly lexicalised form included in the ODNZSL, the sign denotes small round foods, such as a bean, a berry or a grape. The sign also extends to cover food items such as nut, oyster, pea, rice, savory, scallop, and seed.

Polycomponential signs form a large part of the sign language lexicon and express rich visual meaning in a culture-specific way. Brien and Turner (1994) argued that explanatory equivalents or 'proto-definitions' that highlight the simultaneous visual elements in polycomponential signs are therefore essential for learners. As an example, Brien and Turner showed that the sign TOOTHPASTE actually simultaneously encodes information about the toothbrush, the toothpaste tube, and the action of squeezing the tube. The English gloss '(small round) food' in the dictionary entry in figure 5.3 functions as such an an explanatory equivalent in the absence of a suitable hypernym with the same extension, whereas the Te Reo Māori equivalent 'pōkarakara' ('pellet') captures the sense of the round shape but would not typically be used for the kinds of food that are denoted by the sign. Explanatory equivalents are perhaps more effective as a description of the sign's sense, but they do not function well as searchable terms for encoding dictionary users. The entry should therefore 
also contain translational or functional equivalents that enable users to locate the sign. in the case of '(small round) food', a set of hyponyms, i.e. pea, berry, and so on, is provided for this reason.

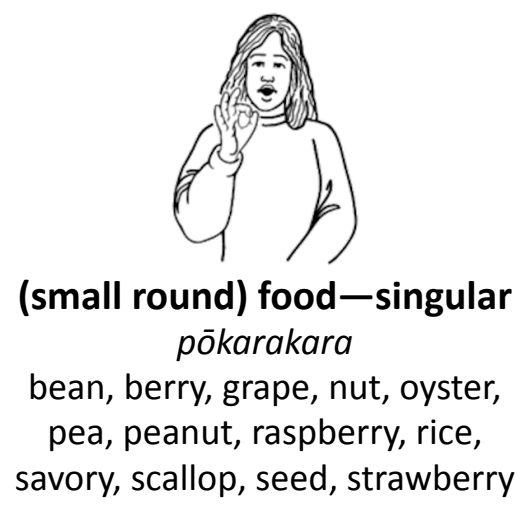

Figure 5.3: A partly-lexicalised sign in which the handshape denotes small round objects, especially food

\subsubsection{Collocational context}

The collocational context of signs is likely to differ significantly from their spoken language equivalents in two respects. First of all, differences in semantic content of culture-bound signs may also be reflected at the level of phraseology. The sign DEAF collocates with other signs to form frequently used sign phrases such as DEAF WORLD, DEAF IDENTITY and DEAF CULTURE (see figure 5.4). These are not common collocations of the English word 'deaf' and as English phrases, the equivalents 'Deaf world', 'Deaf identity' and 'Deaf culture' are therefore potentially uninformative to learners, although the fact that these phrases are included in the ODNZSL as separate headwords will give an indication of their importance and their frequent use in signed discourse.
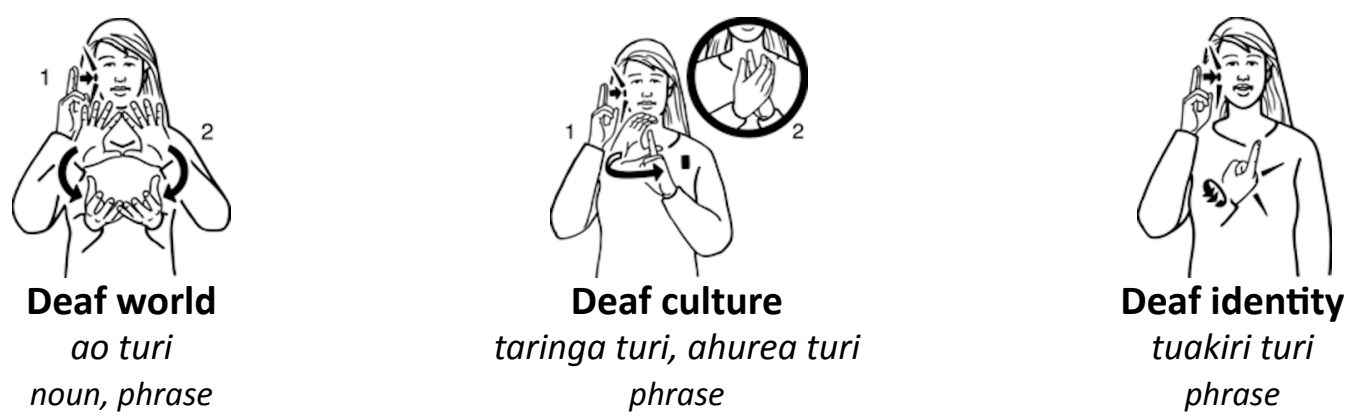

Figure 5.4: Collocations with the sign DEAF as multi-sign headwords in the ODNZSL

Secondly, the differences in modality and structure described in section 2.2.3 - Modality and structure lead to differences in collocational context. The iconic nature of many signs affects collocation patterns: for example, different signs indicating growth are used depending on whether the referent is a person, a plant, or a mass (figure 5.5). 
The simultaneous combination of several independently meaningful elements into a single sign means that some collocation patterns such as verb + noun or verb + adverb actually take place at the morphological level rather than at the syntactic level. For example, verb signs such as GIVE, THROW, or CARRY will typically incorporate different handshapes, use one or both hands, or have a different size of movement to indicate different objects being handled. For the purpose of decoding, the English equivalents will not be problematic here, but they do not provide sufficient information for encoding.

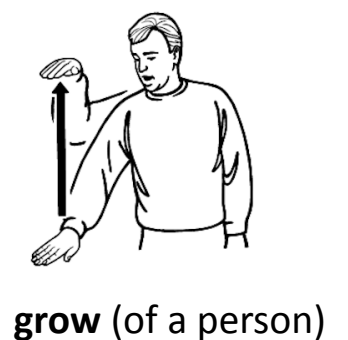

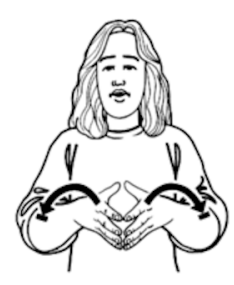

grow, expand (of a mass)

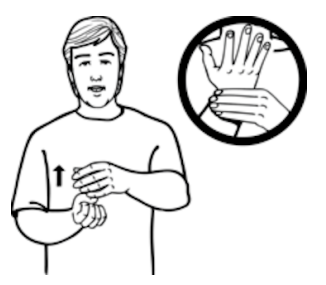

grow (of a plant)

Figure 5.5: Three signs with different collocations-GROW

\subsubsection{Vocabulary type}

A small set of entries in the ODNZSL contain English equivalents that are different in vocabulary type to the sign headword, either because they are drawn from a different register, or because they belong to a different word class.

Figure 5.6 shows two examples of signs that belong to a more informal register than their English main glosses.

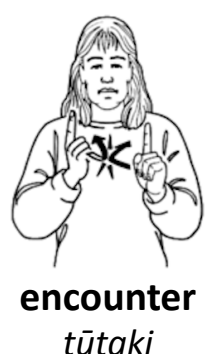

bump into, come across, meet by chance

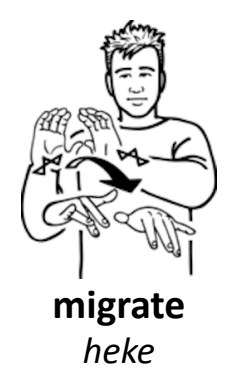

emigrate, immigrate, immigrants, migrants, migration

Figure 5.6: ODNZSL entries with English equivalents drawn from a different register

The choice of English equivalents from more formal registers may be motivated by the need to reflect the main sense of a sign in a one-word gloss, since this gloss not only functions as a (translational) equivalent but also as a written headword, as discussed before. For the sign ENCOUNTER, the more colloquial secondary glosses 'bump into' and 'meet by chance' meet learners' encoding needs more fully. 
English equivalents from technical registers are sometimes applied to sign neologisms that fill previous onomasiological gaps. Such signs often also have non-technical senses based on their iconic or metaphorical properties (see section 2.2.3 - Modality and structure). For example, the sign MIGRATE more literally conveys many people or animals moving from one place to another. The English gloss 'migrate' is useful for encoding dictionary users, but may suggest that such signs are fully lexicalised technical signs when in fact the non-technical senses may be predominant for all but a small part of the Deaf community.

In section 2.2 - Linguistic structure and the lexicon, I referred to the uncertain status of word classes in sign languages. McKee and McKee (2013) mentioned the tagging of word class as one of the larger challenges in making the ODNZSL. They described how many signs, especially signs with an iconic or metaphorical motivation, play different semantic roles in context without varying significantly in form. This situation is reflected in the ODNZSL because many entries include multiple English equivalents from different word classes (e.g. 'bicycle' and 'ride a bicycle'; 'hair dryer' and 'blow dry').

In a few cases, the semantic role of a sign is most effectively shown by an English equivalent from a different word class. The sign shown in figure 5.7 derives from a third person possessive pronoun with a repeated movement indicating emphasis or regularity. The function of this sign is to refer to typical features or characteristics of the referent. The combination of three different word class labels (adjective, verb, and pronoun) as well as English verb, noun and adjective equivalents is potentially confusing for language learners.

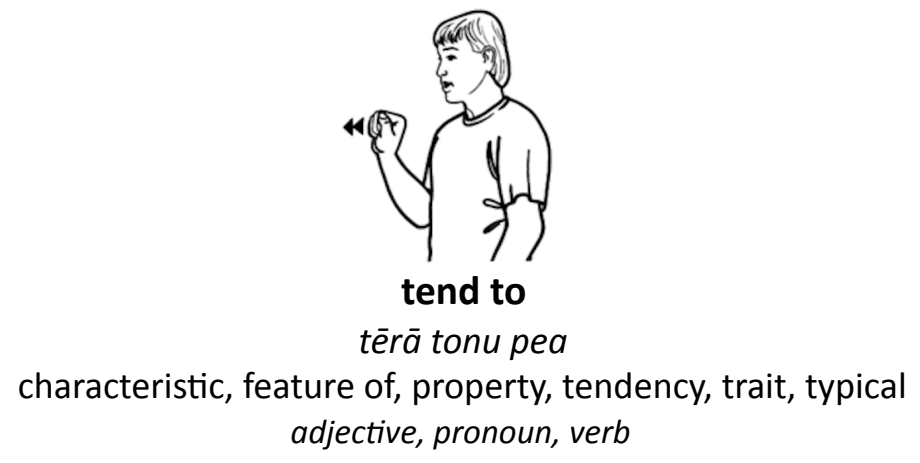

Figure 5.7: ODNZSL entry with English equivalents belonging to a different word class

\subsubsection{Message}

Idiomatic expressions, proverbs and sayings are multi-word constructions with a meaning that is unpredictable from the individual words. Multi-sign fixed expressions are rare in sign languages. According to Johnston and Ferrara (2012), however, idiomaticity is very much 
present in sign languages, but it mostly takes place at the level of lexical constructions, made up of simultaneously combined morphemes. The authors consider any fully-lexicalised sign that has acquired a conventional meaning that is unpredictable from its component parts to be idiomatic. In this view, signs become idiomatic when they move into the category of 'core lexemes' (see section 2.2.2 - The sign language lexicon). In the ODNZSL, the label 'idiom' is reserved for signs that have a figurative meaning and a pragmatic force that is similar to that of a spoken language idiom or phrase.

In some cases, the semantic content of idiomatic NZSL signs is matched by corresponding English idiomatic expressions because the underlying metaphor is similar. This is the case for the ODNZSL entries in figure 5.8.

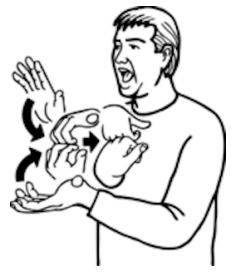

eat my words

kimi i taku patero, kai i aku hamuti

feel stupid, be wrong

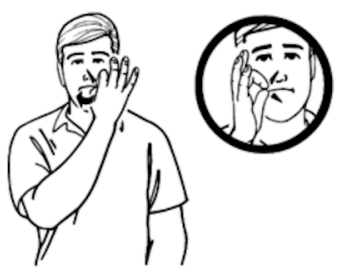

don't tell

kaua e kōrero, kaua e whāki can't tell, keep mum, lips are sealed, remain silent, won't say

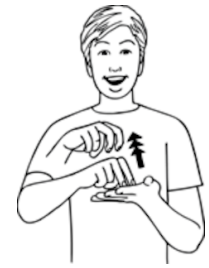

jump for joy

wehe ana i te rekareka delighted, ecstatic, elated, over the moon, very happy

Figure 5.8: Idiomatic expressions with a similar semantic content in NZSL and English

In other cases, signs may include a prototypical metaphor (see section 2.2.3.4 - Metonymy and metaphor) that is not shared with the English equivalents. Figure 5.9 shows some examples.

The three signs on the right-hand side share a movement that indicates something 'falling' or moving away from a previous state (shown in the left-hand column). The choice of an English equivalent for these modified forms is more difficult. While Atkins and Rundell (2008, p. 472) state that "Only the message really matters when it comes to translating idioms and sayings", it may be that an understanding of the underlying visual metaphor would be helpful for sign language learners, especially for vocabulary learning. 


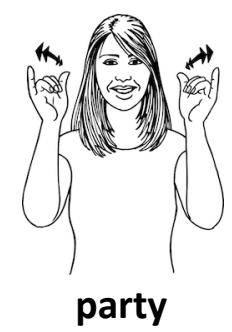

pāti, whakangahau

celebrate, celebration, enjoy, have a good time, have fun

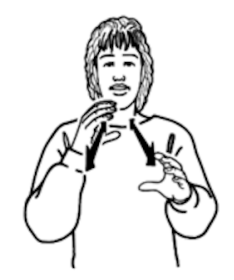

communicate

kōrero, whakawhiti whakaaro

communication

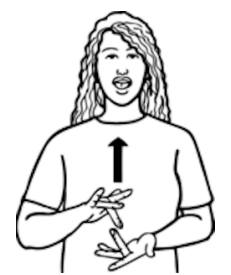

well-behaved

pai

conservative, goody-goody, naive, on one's best behaviour, proper, prudish

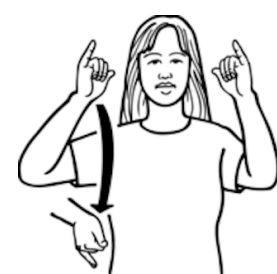

partied out

pi te tero, pau te kaha

not in a party mood, party fatigue, unsociable

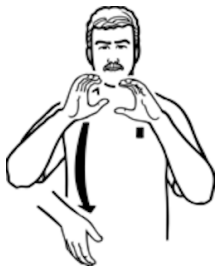

miscommunicate

kāore i mārama tētahi ki tētahi

miscommunication, communication breakdown

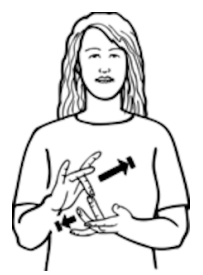

go wild

kua pōrangi noa iho, kua heahea noa iho let one's hair down, let oneself go, loosen up

Figure 5.9: Idiomatic expressions with a different semantic content in NZSL and English

\subsubsection{Function}

Frequency studies of several sign languages (Morford \& MacFarlane, 2003; McKee \& Kennedy, 2006; Johnston, 2012) have shown that the most frequently used signs include a much lower proportion of function signs than is the case for function words in spoken languages. McKee and Kennedy (2006) attributed the smaller number of function signs to the fact that many grammatical functions are not expressed by separate signs, but rather through non-manual features and the use of space (see section 2.2.3.2 - The use of space). Johnston (2012) pointed out that many signs are multifunctional, so that a sign that is used as a grammatical marker in one context may have semantic content in another. Johnston gave as examples the Auslan sign WITH, that can also be used as a verb GO WITH; and the sign BUT, that can also be used as an interjection HOLD ON. There is also uncertainty around the status of pointing signs as pronouns (see section 2.2.4.2 - Indexicals or pointing signs). 
ODNZSL entries for function signs are most likely to be problematic for learners when the equivalents suggest a function or use that is different from their use in NZSL. For example, the sign THROUGH shares some functions with the English word 'through', but is also often used as a conjunction with a sense of causation (e.g. becoming deaf 'through' rubella) or as a verb ('go through'); and the sign OVER can be used in a similar way to the English 'about'.

A further problem may be that sign language learners, especially beginner learners, are unaware that some highly frequent English conjunctions, auxiliaries or other function words are used much less frequently in sign language. They may therefore over-use signs such as AND or the auxiliary WILL in their sign production.

These problems would not be solved by adding signed definitions, since as explanations of word meaning, definitions are not useful for semantically empty function words. Other strategies are required to facilitate learners' understanding of the grammatical functions of certain signs. In the ODNZSL, this includes the provision of signed examples as well as usage notes and grammatical labels. Some grammatical categories are also provided as searchable topics (e.g. pronouns) or as separate sections of the website (e.g. numerals).

\subsection{Examples}

The previous section has shown that equivalents by themselves may not provide sufficient information to help users identify the correct sense and usage of the word or sign. One of the main parts of the dictionary entry that is intended to address these user needs is the example.

The original function of examples in descriptive and historical dictionaries was mainly one of attestation: proving that a particular word is actually used in the language by showing a quotation from a text source (Atkins \& Rundell, 2008). Examples have two other main functions, both well documented in particular in the context of learner's dictionaries, whether monolingual or bilingual (see e.g. Fox, 1987; Nesi, 1996; Prinsloo \& Gouws, 20oo; Atkins \& Rundell, 2008). Firstly, examples can help decoding users to understand the meaning of the headword better. Examples elucidate meaning by illustrating usage and disambiguating between senses for polysemous words. Secondly, examples are especially important for encoding users, who not only need to identify the correct sense of a target word, but also need to use it correctly. For this purpose, examples need to show the word in a typical context of use, illustrating grammatical structure and collocation, register, regional distribution et cetera. 
Several studies have tried to assess the impact of examples by comparing language learners' performance after using dictionary entries with and without examples. Laufer (1992) found that comprehension of new words was better when both definitions and illustrative examples were provided than with examples on their own. Gumming, Cropp, and Sussex (1994) found that learners preferred the fullest possible dictionary information, including both (full sentence) definitions and examples, but with examples being rated more important than definitions. However, no significant differences were found in either production or comprehension tests with different formats of dictionary entry. Similarly, Nesi (1996) found that examples had no effect on language production. Al-Ajmi (2008) noted that examples had a negative impact in a reading comprehension / translation task, with learners making more errors after consulting dictionary entries with both definitions and examples than when they only used the definition. Frankenberg-Garcia (2012) argued that Al-Ajmi's findings might have been caused by situations where examples provided more semantic information than the core meaning expressed in the definition. In her own study, Frankenberg-Garcia found that with a single example, learners did not perform as well as with a definition, but when multiple full-sentence examples were provided, learners were able to draw conclusions on the basis of patterns in the examples alone and had improved scores on both comprehension and production.

The inconclusive findings from these studies suggest that there are other factors that determine the usefulness of examples. Nesi (1996) posed three possible factors: firstly, the learners' patterns of dictionary use may mean that they did not locate or use the information available to them in the examples; secondly, the examples did not provide the right information; or thirdly, the method used to test learners' production was at fault. These factors are relevant to evaluating any element of the dictionary entry and will therefore be borne in mind for the evaluation of signed definitions in a later chapter (Chapter 7 - From folk definition to dictionary definition). In the current chapter, the quality and source of examples are relevant.

Atkins and Rundell (2008) formulated three basic criteria for good examples, stating that they should be natural and typical; informative; and intelligible. There has been considerable debate on the kind of example most likely to meet these criteria. Initial debates centred on a comparison between examples extracted from a corpus and lexicographer-created examples. Fox (1987), for instance, argued that corpora were a better guide to authentic and typical usage than intuition, and that corpus examples should be edited as little as possible. By 
contrast, Cowie (1989) maintained that it was difficult to find authentic corpus examples that would suit both encoding and decoding purposes and be easily understood by learners.

The effect of example format on learner performance has not been conclusively established: Laufer (1992) and Nesi (1996) found in favour of created examples, but later studies criticised these findings, arguing that both learners and teachers prefer authentic examples when surveyed (Potter, 1998) and that the nature of the tasks given to learners in these studies did not reflect typical learning contexts (Frankenberg-Garcia, 2012). Later authors therefore argued for a middle ground, based on the function of the example rather than its source. Potter (1998) conceded that absolute beginner users may benefit from created examples and that some editing of corpus examples for length and potentially distracting information might be required. Prinsloo and Gouws (2000) proposed systematic editing of corpusinformed examples so that each example addresses the needs of at least one target user group. Atkins and Rundell (2008) argued that it was extremely rare to locate examples in a corpus that would meet all three of their criteria and that some editing would therefore be required in most cases, except for the purpose of attestation. In active bilingual dictionaries, examples should nearly always be created to maximise intelligibility and informativeness.

Humblé $(1998,2001)$ not only considered the function and user groups of dictionaries as a whole, but argued that different entries called for different examples. Focusing on language learners consulting the dictionary for the purpose of encoding, Humblé distinguished between beginner, intermediate and advanced learners. Each group is characterised not only by different language proficiency (command of vocabulary, syntax and lexicogrammar), but also by different lookup behaviour. Whereas beginner learners tend to concentrate on high frequency vocabulary, advanced learners rarely look up these words, instead focusing on low frequency or technical words. Humblé therefore argued that authentic corpus examples were most suitable to illustrate low frequency words, providing rich information on collocation and usage in a form accessible to advanced learners. For high frequency vocabulary, intelligibility of the example would be a priority, calling in most cases for created or heavily edited examples. Uniquely, Humblé also proposed that intermediate learners have specific need for mid-frequency vocabulary and require both intelligibility and natural, authentic usage information.

Frankenberg-Garcia (2012) recommended that multiple examples focused explicitly on either encoding or decoding should be given, with an emphasis on decoding needs for low 
frequency vocabulary and on encoding needs for high frequency words. Frankenberg-Garcia suggested that with this approach, examples on their own would provide sufficient assistance to learners so that definitions would in theory no longer be required. However, she also pointed out that in practice, locating the right information in examples and distinguishing between examples for decoding and examples for encoding would be very difficult. Since Frankenberg-Garcia also stressed the importance of using full-sentence examples carefully selected to illustrate meaning, this approach is not incompatible with the proposed addition of folk definitions to sign language dictionary entries that already contain corpus-based examples, such as the ODNZSL. In some ways, folk definitions could be treated as a specific kind of explanatory example rather than an attempt to provide a traditional dictionary definition. In Chapter 6 - Folk definitions, I will discuss the nature of folk definitions in more detail. First, I will examine current examples in electronic sign language dictionaries.

\subsubsection{Examples in sign language dictionaries}

Since many early sign language dictionaries took the form of rudimentary glossary lists (see section 3.1 - History and purposes of sign language dictionaries), usage examples were rarely provided. In descriptive sign language dictionaries in print form such as the Dictionary of British Sign Language/English (Brien, 1992) and the Dictionary of NZSL (Kennedy et al., 1997), written English examples were provided to indicate which of the sense(s) of the English glosses corresponded with the meaning of the sign. Such examples, however, by their very nature could not illustrate grammatical and usage features of the sign in context.

In electronic sign language dictionaries, signed examples can be presented directly on video. The source and functions of video examples have been described for the Danish Sign Language dictionary (Kristoffersen, 2010), and for the ODNZSL (McKee \& McKee, 2013). Both these dictionaries serve as descriptive works and as pedagogical resources. Video examples in these dictionaries have therefore been used both as attestation and to present the sign in a typical context for decoding and encoding purposes, particularly for language learners.

In section 3.4.9 - Corpora and corpus-based lexicography, I referred to the increasing role of corpora in sign language lexicography. Both the Danish Sign Language dictionary and the ODNZSL made use of sign language corpora to extract authentic examples where possible. The small size of sign language corpora meant that no suitable corpus evidence was available in about 30\% of cases for the ODNZSL (McKee \& McKee, 2013) and 45\% of cases for the Danish Sign Language Dictionary (Kristoffersen, 2010). In addition to corpus-derived 
examples, both dictionaries therefore also contain examples created by Deaf members of the lexicography team. As discussed in the previous section, although this mixture of corpusbased and created examples is a pragmatic compromise motivated by the small size of sign language corpora, it is not an unusual approach especially in pedagogical lexicography. For attestation purposes, authentic examples are required, but for all other purposes, at least some editing of corpus examples is usually required.

Both the ODNZSL and the Danish Sign Language Dictionary based their selection and editing of corpus examples, and formulation of examples when there was no available corpus data, on Atkins and Rundell's (2008) criteria that examples should be natural and typical, informative, and intelligible. Whether the resulting edited examples in the ODNZSL meet these criteria is difficult to evaluate. The shortage of corpus data and linguistic descriptions of the grammar of sign languages mean that it is not possible in many cases to say with certainty whether a particular usage is natural or typical. The intelligibility of examples has not been tested with dictionary users, but the user study in section 4.6.3.4 - Information viewed in the dictionary entry indicated that intermediate learners generally found the examples easy to understand even though they were too difficult to follow for beginner learners. Two features that increase intelligibility are written English translations of the examples, and cross-links to the dictionary entries for other signs used in the examples.

To assess the kind of information that is contained in the examples, e.g. whether they provide semantic, contextual or grammatical support, would require a systematic analysis of the examples as a whole, similar to that provided by Hiles (2011) of examples in South African school dictionaries. Such an analysis would show more clearly which user needs are currently met by the examples and which would be better met by definitions. While a full analysis is beyond the scope of this thesis, I will here provide some illustrations of examples in the ODNZSL that provide different kinds of information.

\subsubsection{Elucidating meaning}

\section{Examples as definitions}

In a very few cases, the example provides an explanation of the core sense of the sign in a way that is similar to a (context-free) definition. Figure 5.10 shows the example for the sign DIRECTIONAL-VERB, a technical sign that is included in the ODNZSL because of its relevance to sign language linguistics. This example is informative but appears to be modelled on a formal dictionary defining style, making it a less natural and typical NZSL utterance. 


\section{Example 1}

A directional verb is a sign that can inflect according to who is performing the action.

Figure 5.10: An example providing a definition of the sign DIRECTIONAL-VERB

\section{Distinguishing senses}

Figure 5.11 shows two examples that help dictionary users differentiate between two different senses of the sign DEAF (http://nzsl.vuw.ac.nz/signs/887). This sign phrase, consisting of the component signs FULLY (http://nzsl.vuw.ac.nz/signs/889) and DEAF (http://nzsl.vuw.ac.nz/ signs/4656), can either refer to a person who is 'fully immersed' in the signing Deaf community, or to a person who has a 'complete' or 'profound' hearing loss. The contextual support provided in these examples both illustrates and distinguishes between the two senses and shows typical NZSL utterances in which the sign might be used.

FULLY-DEAF

Example 1

She is culturally Deaf. She's very involved in the Deaf community and is a fluent signer.

\section{Example 2}

I had an ear infection, and in one week, my hearing worsened and I was totally deaf.

Figure 5.11: Examples distinguishing between two senses of the sign phrase (CULTURALLY/ TOTALLY) DEAF

\section{Overcomplete context}

The example in figure 5.12 illustrates the meaning of the idiomatic sign HUMILIATED by providing a scenario that might cause the signer to experience a sense of humiliation. Although the example provides a suitable context for understanding the meaning of the sign, Potter (1998) and Atkins and Rundell (2008), amongst others, warned against the risk that such invented examples may be over-explanatory and as a result lose the ability to provide learners with information on natural or typical usage. 
HUMILIATED

\section{Example 1}

I was trying to pull the door open but a man came along and casually pushed it open-I felt humiliated.

Figure 5.12: A created example providing a context for the sign HUMILIATED

\subsubsection{Grammatical support}

Many of the examples in the ODNZSL provide grammatical support that is especially valuable for encoding learners. This role of examples is clearest in the case of function signs, where semantic content does not need to be illustrated. Figure 5.13 shows two examples illustrating that the sign OFF is frequently used as a verb (as reflected in the translational equivalents 'get off' and 'remove') and that, just as in English, the collocation 'off work' is typical in NZSL.

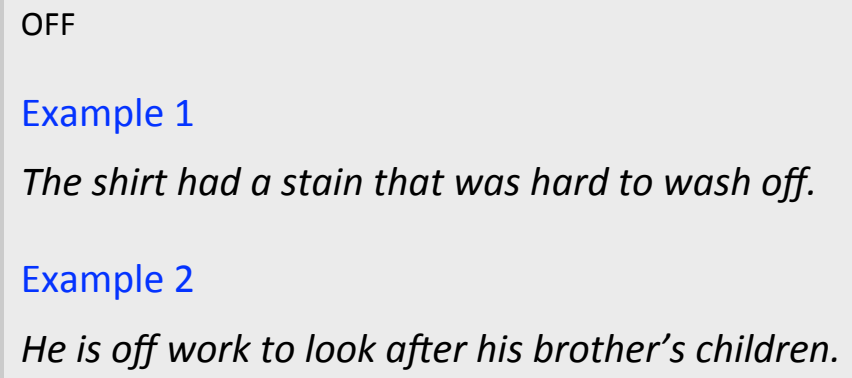

Figure 5.13: Examples with grammatical support illustrating uses of the sign OFF

\subsubsection{Cultural support}

According to McKee and McKee (2013, p. 518) corpus-based examples are especially valuable in sign language dictionaries because they illustrate "Deaf cultural frames that underpin the lexicon in use". In other words, by showing signs in a typical context, authentic examples can provide learners with information about the shared experiences and values of Deaf people.

Figure 5.14 shows examples for the sign HEARING and for a variant form with the same meaning. The examples illustrate two typical contexts in which these signs are used: firstly, to describe individuals whose hearing status is relevant because they have direct contact with the Deaf community, e.g. family members or close friends; and secondly, to refer to the wider majority society in general in contrast with the minority Deaf community-hence, a 'hearing' club as opposed to a club run by the Deaf community. These examples are important as models for encoding, but are not immediately useful for decoding learners. The addition of a usage note stating that these signs "describe people who are not Deaf rather than the act of 
hearing sound" is an acknowledgment that the English equivalent 'hearing' is potentially misleading and that the examples do not directly clarify the meaning of the sign for learners who are not already aware of its meaning.

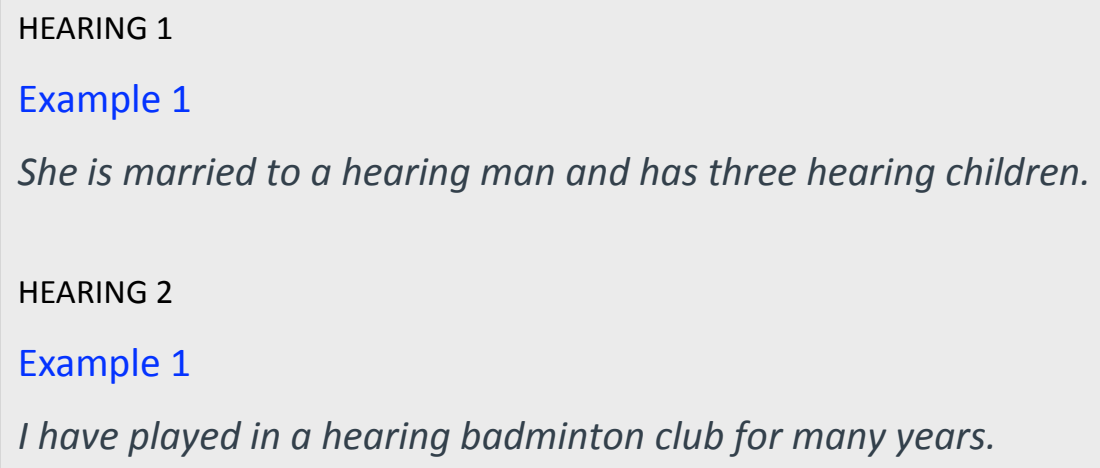

Figure 5.14: Examples illustrating Deaf cultural frames for the signs HEARING 1 and HEARING 2

Minaeva (1992) and Potgieter (2012) have both argued that examples that include implicit target language cultural information are distracting for learners. Instead of being able to focus on the sense and usage of the target word, learners spend time 'unpacking' the unfamiliar cultural references. While conveying culturally rich information to learners is a crucial function of sign language dictionaries, an example is therefore not the ideal place for such information to be included. This is especially true for examples illustrating the usage of signs that have no culture-specific semantic content. For example, learners who are trying to understand the meaning and use of the sign PLAIN (figure 5.15) would need to understand that the sign INTERPRETER refers specifically to sign language interpreters and that sign language interpreters' hands need to be clearly visible against the background of their clothing. The useful collocational information that the sign PLAIN is typically used to describe clothing may get lost in the process of trying to understand the implicit frames in the example.

\section{PLAIN}

Example 1

Interpreters should wear plain tops so that they can be seen clearly.

Figure 5.15: An example for the sign PLAIN containing potentially distracting implicit cultural information 


\subsection{Research question}

The overview of equivalents and examples of use in the ODNZSL has identified some categories of signs that are inadequately described by the current information. Although these categories provide a starting point for the selection of signs for which definitions could be developed, it is important to confirm that the current dictionary entries for these signs are problematic for learners in encoding, decoding and/or vocabulary learning. A test was developed to assess the sufficiency of current dictionary information for these language purposes, by simulating language activities that would require the target group of hearing, adult, intermediate L2 learners to use the dictionary information. The research question this test was designed to answer is:

- Which signs or sign categories could benefit most from signed (L2) definitions to provide additional information that (intermediate) learners of NZSL need to understand and use these signs?

\subsection{Methodology of dictionary tests}

In designing the test, I considered a number of models from previous studies that have assessed the effectiveness of different types of dictionary information. These tests have mainly used what Tono (2001) referred to as 'quasi-experimental' designs: they were quantitative studies of a relatively small set of language learners from a homogenous background (often drawn from a single course at the same institution), using either a pre-test post-test format with one or more groups of students (e.g. Nesi, 1996) or a between-group comparison with or without a control group (e.g. Laufer, 1992; Laufer \& Melamed, 1994).

Usually, tests have taken place as part of normal classroom activities to ensure ecological validity, although in some studies, the tasks set in order to control study variables may be somewhat unnatural. For example, Nesi and Meara (1994) acknowledged that in a classroom situation, students would not be expected to immediately use unfamiliar vocabulary that was presented to them out of context.

Tests that assessed the effect on both comprehension and production have generally included different decoding and encoding tasks with the same test items, although FrankenbergGarcia (2012) tested lower frequency vocabulary for comprehension and higher frequency vocabulary which was already partly familiar to her participants for production. 
Decoding activities have included translation of the test item into the student's L1 (Laufer, 1992; Al-Ajmi, 2008), selecting the correct sense from a multiple-choice list (Laufer \& Melamed, 1994; Frankenberg-Garcia, 2012); or deciding whether the test item was used correctly in a sentence (Gumming, Cropp, \& Sussex, 1994).

The common encoding activity of producing a sentence with the target word after dictionary consultation (e.g. Laufer, 1992; Laufer \& Melamed, 1994) has been criticised as being unrealistic for learners to be able to carry out effectively for lexical items that they are completely unfamiliar with. Learners may also produce sentences that do not provide any information about whether they have correctly understood the target word. Alternative encoding activities have included the requirement to use both the target item and another word in a sentence (Nesi \& Meara, 1994), and identifying and correcting errors in a sentence with the target item (Frankenberg-Garcia, 2012).

The design for the current test was influenced by the fact that the test needed to fulfil two purposes. Firstly, it should identify candidate signs for the development of a small number of signed definitions in the next study (see Chapter 6 - Folk definitions). For this purpose, a test with a single group of participants would be sufficient. However, once signed definitions had been developed, the effect of including the definitions for these items would also need to be measured. Numbers of students in intermediate NZSL classes were likely to be small and I therefore chose a between-subjects design, with the first group of students' test results serving as a pre-test for the second group and the items that did not receive the treatment serving as a control to ensure that the two student groups were matched. The method and results of the first test group will be described in the next section. The repeat test after the treatment will be addressed in section 7.3 - Testing the effectiveness of dictionary definitions.

Since the ODNZSL already includes bilingual equivalents in English, a translation task was not suitable to assess comprehension. I decided on an error correction format for both the comprehension and the production section, to limit the signed output students would have to produce. Following the test design in the study of Gumming, Cropp, and Sussex (1994), the test contained sentences with an error as well as error-free sentences. As in the study by Frankenberg-Garcia (2012), participants not only had to identify whether a sentence was correct or not, but also had to correct the error. 


\subsection{Method}

\subsubsection{Materials}

Test items were selected from existing entries in the ODNZSL, based on the following criteria:

- Test items should be drawn from those categories that were identified in section 5.2.5 Non-equivalence in the ODNZSL as most likely to exhibit partial or zero equivalence.

- The test should include a cross-section of lexemes of different types, including marginal lexemes and lexemes that may have originated from the non-core and non-native lexicon. As was discussed in section 2.3 - Summary and discussion, the structure and semantics of these types of lexeme would be least familiar to learners whose native language is a spoken language.

- The test should include some items where the iconic or metaphoric motivation of the sign might compensate for incomplete description in the dictionary entry.

- Test items should be less familiar to intermediate learners of NZSL.

- The test should include a mixture of encoding and decoding issues, including distinguishing between different senses or uses of polysemous signs.

In total 463 sign lemmas (approximately $8 \%$ of the total number of entries in the ODNZSL) met these criteria. For the purpose of achieving a balanced test, 50 signs were selected and grouped into six different categories. Table 5.1 describes these categories; tables 5.2 through to 5.7 list the signs selected in each category and the corresponding entries in the ODNZSL. It should be noted that some signs could fit into multiple categories: for example, signs that were selected because they demonstrate a potentially unfamiliar collocation pattern may also be culture-bound. There is also considerable overlap between the idiomatic and metaphoric categories. Categories 5 and 6 in particular contained a mixture of grammatical and collocational issues. 


\begin{tabular}{|c|c|c|c|}
\hline $\begin{array}{l}\text { Category } \\
\text { number }\end{array}$ & Category name & Description & $\begin{array}{l}\text { Number of } \\
\text { test items }\end{array}$ \\
\hline 1 & Culture-bound & $\begin{array}{l}\text { Signs that reflect Deaf cultural referents, } \\
\text { experiences and behaviours }\end{array}$ & 10 \\
\hline 2 & Idiomatic & $\begin{array}{l}\text { Signs with a figurative meaning that is } \\
\text { unpredictable from their component parts }\end{array}$ & 10 \\
\hline 3 & Polysemous & $\begin{array}{l}\text { Signs that have multiple senses, including partially } \\
\text { specified polycomponential signs }\end{array}$ & 5 \\
\hline 4 & $\begin{array}{l}\text { Metaphoric / } \\
\text { metonymic }\end{array}$ & $\begin{array}{l}\text { Signs that include a visual metaphor (recognition } \\
\text { of which may contribute to learners' } \\
\text { understanding of the sign) }\end{array}$ & 5 \\
\hline 5 & $\begin{array}{l}\text { Vocabulary type / } \\
\text { word class }\end{array}$ & $\begin{array}{l}\text { A mixture of grammatical issues, including signs } \\
\text { that either belong to a different register or to a } \\
\text { different word class from the English equivalents }\end{array}$ & 10 \\
\hline 6 & Other & $\begin{array}{l}\text { Function signs and other grammatical and } \\
\text { collocational issues }\end{array}$ & 10 \\
\hline
\end{tabular}

Table 5.1: Categories of test items 


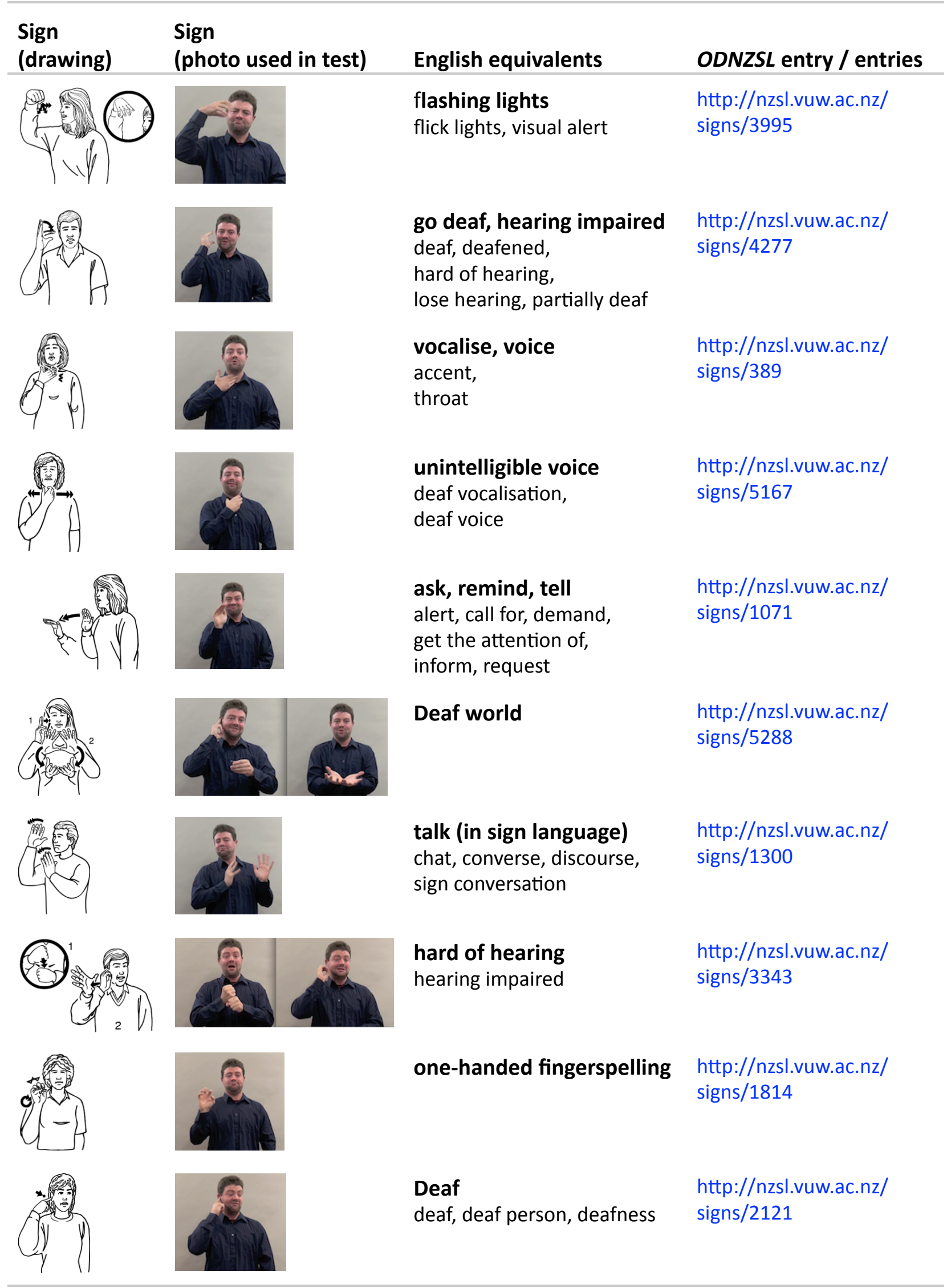

Table 5.2: Test items in category 1 -Culture-bound 


\begin{tabular}{|c|c|c|c|}
\hline $\begin{array}{l}\text { Sign } \\
\text { (drawing) }\end{array}$ & $\begin{array}{l}\text { Sign } \\
\text { (photo used in test) }\end{array}$ & English glosses & ODNZSL entry / entries \\
\hline & & $\begin{array}{l}\text { face up to } \\
\text { face }\end{array}$ & $\begin{array}{l}\text { http://nzsl.vuw.ac.nz/ } \\
\text { signs/329 }\end{array}$ \\
\hline & & $\begin{array}{l}\text { keep inside oneself } \\
\text { bottle up, keep it to oneself, } \\
\text { live with, suppress }\end{array}$ & $\begin{array}{l}\text { http://nzsl.vuw.ac.nz/ } \\
\text { signs/1157 }\end{array}$ \\
\hline & & $\begin{array}{l}\text { whoops } \\
\text { misstep, oops, what am I doing? }\end{array}$ & $\begin{array}{l}\text { http://nzsl.vuw.ac.nz/ } \\
\text { signs/5109 }\end{array}$ \\
\hline & & $\begin{array}{l}\text { eat my words } \\
\text { feel stupid, be wrong }\end{array}$ & $\begin{array}{l}\text { http://nzsl.vuw.ac.nz/ } \\
\text { signs/5176 }\end{array}$ \\
\hline & & $\begin{array}{l}\text { one-up } \\
\text { out-do }\end{array}$ & $\begin{array}{l}\text { http://nzsl.vuw.ac.nz/ } \\
\text { signs/5052 }\end{array}$ \\
\hline & & $\begin{array}{l}\text { silly me } \\
\text { doh, kick oneself, oops }\end{array}$ & $\begin{array}{l}\text { http://nzsl.vuw.ac.nz/ } \\
\text { signs/5204 }\end{array}$ \\
\hline & & $\begin{array}{l}\text { been to, have experience of } \\
\text { been with, done that, } \\
\text { touch, visit }\end{array}$ & $\begin{array}{l}\text { http://nzsl.vuw.ac.nz/ } \\
\text { signs/4794 }\end{array}$ \\
\hline & & $\begin{array}{l}\text { multitask } \\
\text { divided attention }\end{array}$ & $\begin{array}{l}\text { http://nzsl.vuw.ac.nz/ } \\
\text { signs/5451 }\end{array}$ \\
\hline & & $\begin{array}{l}\text { oh good } \\
\text { looking forward to, yay }\end{array}$ & $\begin{array}{l}\text { http://nzsl.vuw.ac.nz/ } \\
\text { signs/4899 }\end{array}$ \\
\hline & & $\begin{array}{l}\text { I was wrong, (you) got me } \\
\text { eat my words, fell for it, } \\
\text { feel silly, shamefaced, sheepish, } \\
\text { sucked in }\end{array}$ & $\begin{array}{l}\text { http://nzsl.vuw.ac.nz/ } \\
\text { signs/6089 }\end{array}$ \\
\hline
\end{tabular}

Table 5.3: Test items in category 2-Idiomatic 


\begin{tabular}{|c|c|c|c|}
\hline $\begin{array}{l}\text { Sign } \\
\text { (drawing) }\end{array}$ & $\begin{array}{l}\text { Sign } \\
\text { (photo used in test) }\end{array}$ & English glosses & ODNZSL entry / entries \\
\hline & & $\begin{array}{l}\text { confidential, private } \\
\text { confidentiality, in confidence, } \\
\text { in private, keep quiet, } \\
\text { privacy, privately }\end{array}$ & $\begin{array}{l}\text { http://nzsl.vuw.ac.nz/ } \\
\text { signs/267 }\end{array}$ \\
\hline & & $\begin{array}{l}\text { plain } \\
\text { dull, simple, tasteless }\end{array}$ & $\begin{array}{l}\text { http://nzsl.vuw.ac.nz/ } \\
\text { signs/266 }\end{array}$ \\
\hline & & $\begin{array}{l}\text { (small round) food-plural } \\
\text { beans, berries, cherries, grapes, } \\
\text { nuts, oysters, peas, radishes, } \\
\text { rice, scallops, seeds, } \\
\text { strawberries }\end{array}$ & $\begin{array}{l}\text { http://nzsl.vuw.ac.nz/ } \\
\text { signs/3137 }\end{array}$ \\
\hline & & $\begin{array}{l}\text { develop, grow } \\
\text { development, expand, } \\
\text { expansion, growth, increase }\end{array}$ & $\begin{array}{l}\text { http://nzsl.vuw.ac.nz/ } \\
\text { signs/3567 }\end{array}$ \\
\hline & & $\begin{array}{l}\text { centre, Central (zone), } \\
\text { middle } \\
\text { central }\end{array}$ & $\begin{array}{l}\text { http://nzsl.vuw.ac.nz/ } \\
\text { signs/4798 }\end{array}$ \\
\hline & & $\begin{array}{l}\text { grease } \\
\text { fat, fatty, greasy, oily }\end{array}$ & $\begin{array}{l}\text { http://nzsl.vuw.ac.nz/ } \\
\text { signs/5826 }\end{array}$ \\
\hline & & $\begin{array}{l}\text { thing } \\
\text { stuff, substance }\end{array}$ & $\begin{array}{l}\text { http://nzsl.vuw.ac.nz/ } \\
\text { signs/5824 }\end{array}$ \\
\hline & & $\begin{array}{l}\text { fabric } \\
\text { cloth, cotton, material, nylon, } \\
\text { silk, wool }\end{array}$ & $\begin{array}{l}\text { http://nzsl.vuw.ac.nz/ } \\
\text { signs/5825 }\end{array}$ \\
\hline & & $\begin{array}{l}\text { fine } \\
\text { delicate, soft }\end{array}$ & $\begin{array}{l}\text { http://nzsl.vuw.ac.nz/ } \\
\text { signs/4052 }\end{array}$ \\
\hline & & $\begin{array}{l}\text { sand, soil } \\
\text { dirt, earth }\end{array}$ & $\begin{array}{l}\text { http://nzsl.vuw.ac.nz/ } \\
\text { signs/1507 }\end{array}$ \\
\hline & & $\begin{array}{l}\text { texture } \\
\text { feel }\end{array}$ & $\begin{array}{l}\text { http://nzsl.vuw.ac.nz/ } \\
\text { signs/5828 }\end{array}$ \\
\hline
\end{tabular}

Table 5.4: Test items in category 3-Polysemous 


\section{Sign}

\section{Sign (drawing) (photo used in test)}

English glosses

ODNZSL entry / entries

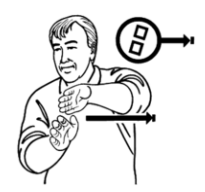

N/A (alternative sign

put aside

shown in the test item)

apart from, defer, except,

http://nzsl.vuw.ac.nz/

let alone, next topic
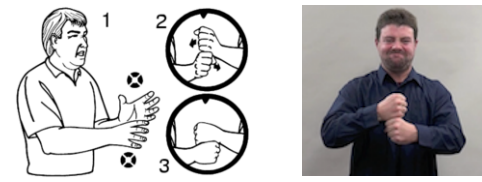

exasperated with

http://nzsl.vuw.ac.nz/

be infuriated, exasperating,

get on one's nerves

signs/1878

N/A (alternative sign contradict oneself

change one's story, falsify, lie

http://nzsl.vuw.ac.nz/ shown in the test item)

signs/5149

disconnect

lose contact, lose touch, out of touch

http://nzsl.vuw.ac.nz/

signs/5213

hook up

http://nzsl.vuw.ac.nz/

get together (couple), pick up

Table 5.5: Test items in category 4-Metaphoric / metonymic 


\begin{tabular}{|c|c|c|c|}
\hline $\begin{array}{l}\text { Sign } \\
\text { (drawing) }\end{array}$ & $\begin{array}{l}\text { Sign } \\
\text { (photo used in test) }\end{array}$ & English glosses & ODNZSL entry / entries \\
\hline & & $\begin{array}{l}\text { time span } \\
\text { duration, from ... to, } \\
\text { length of time, period of time }\end{array}$ & $\begin{array}{l}\text { http://nzsl.vuw.ac.nz/ } \\
\text { signs/492 }\end{array}$ \\
\hline & & $\begin{array}{l}\text { strongly, very } \\
\text { marked, strong, very much }\end{array}$ & $\begin{array}{l}\text { http://nzsl.vuw.ac.nz/ } \\
\text { signs/3266 }\end{array}$ \\
\hline & & $\begin{array}{l}\text { off } \\
\text { get off, remove }\end{array}$ & $\begin{array}{l}\text { http://nzsl.vuw.ac.nz/ } \\
\text { signs/555 }\end{array}$ \\
\hline & & $\begin{array}{l}\text { Deaf } \\
\text { culturally Deaf, } \\
\text { profoundly deaf, totally deaf }\end{array}$ & $\begin{array}{l}\text { http://nzsl.vuw.ac.nz/ } \\
\text { signs/887 }\end{array}$ \\
\hline & & $\begin{array}{l}\text { over } \\
\text { about }\end{array}$ & $\begin{array}{l}\text { http://nzsl.vuw.ac.nz/ } \\
\text { signs/5415 }\end{array}$ \\
\hline & & hearing & $\begin{array}{l}\text { http://nzsl.vuw.ac.nz/ } \\
\text { signs/2117 }\end{array}$ \\
\hline & & $\begin{array}{l}\text { to } \\
\text { towards }\end{array}$ & $\begin{array}{l}\text { http://nzsl.vuw.ac.nz/ } \\
\text { signs/2299 }\end{array}$ \\
\hline & & $\begin{array}{l}\text { decrease } \\
\text { decline, die down, drop, } \\
\text { dwindle, fall, lessen, reduce }\end{array}$ & $\begin{array}{l}\text { http://nzsl.vuw.ac.nz/ } \\
\text { signs/1294 }\end{array}$ \\
\hline & Not used in test & $\begin{array}{l}\text { decrease } \\
\text { decline, die down, drop, } \\
\text { dwindle, fall, lessen, reduce }\end{array}$ & $\begin{array}{l}\text { http://nzsl.vuw.ac.nz/ } \\
\text { signs/249 }\end{array}$ \\
\hline & & exist & $\begin{array}{l}\text { http://nzsl.vuw.ac.nz/ } \\
\text { signs/5837 }\end{array}$ \\
\hline & & $\begin{array}{l}\text { have } \\
\text { possess }\end{array}$ & $\begin{array}{l}\text { http://nzsl.vuw.ac.nz/ } \\
\text { signs/1376 }\end{array}$ \\
\hline & & then & $\begin{array}{l}\text { http://nzsl.vuw.ac.nz/ } \\
\text { signs/5970 }\end{array}$ \\
\hline
\end{tabular}

Table 5.6: Test items in category 5-Vocabulary type / word class 


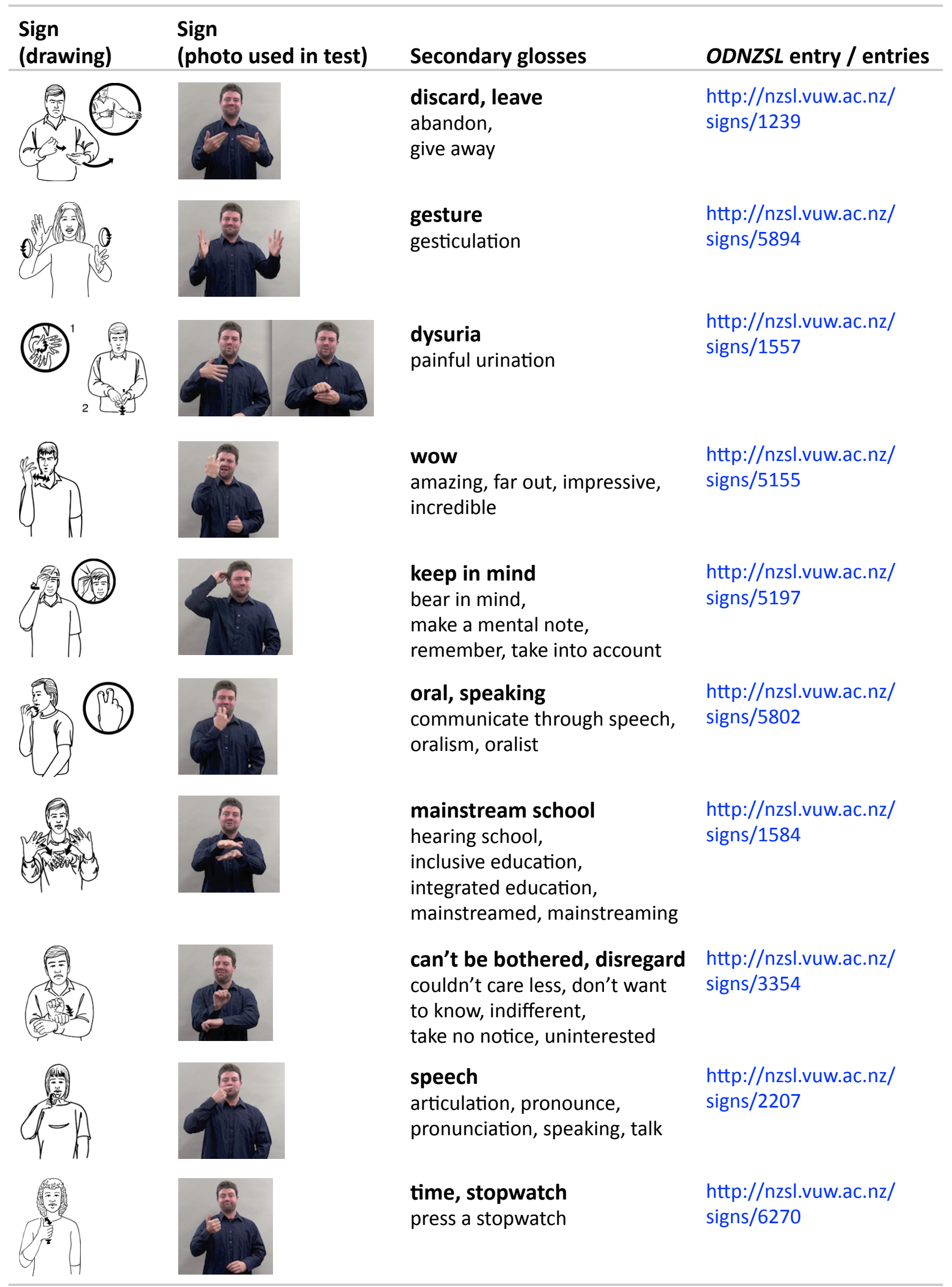

Table 5.7: Test items in category 6-Other 


\subsubsection{Developing the test instrument}

A computer-based test instrument was necessary because both signed test items and participants' signed responses required the inclusion of video material. Participants would also be expected to refer to information from an online dictionary. I used the May 2014 version of Qualtrics survey software (Qualtrics, 2016) to create the test instrument. The Qualtrics platform allowed me to integrate multimedia into the test, to randomise test items, to administer the test remotely and to report on test results anonymously.

The 50 selected test items were grouped into five blocks of to items so that each block contained the same number of items from each category.

Each block was further divided into two sections of five questions each, simulating decoding (comprehension) and encoding (production) tasks. Items from categories 1, 2, and 3 were included in the comprehension section, and items from categories 4, 5, and 6 made up the production questions.

In the comprehension section, an NZSL sentence was presented on video and a translation of this sentence into English was either correct or contained an error. In the production section, a written English sentence was shown and participants had to judge whether a translation of this sentence into NZSL was correct or contained an error. Figure 5.16 and figure 5.17 show examples of the comprehension and production question formats respectively.

Since the objective of the test was to assess whether learners were able to decode and encode a target sign correctly on the basis of information in the signs's dictionary entry, it was important that this information was actually viewed. Due to the length of dictionary entries in the ODNZSL, full entries could not be embedded in the test. Instead, each question therefore directed participants to click on a still image of the target sign to be taken to the relevant ODNZSL entry.

Production questions required participants to film themselves signing their response. Unfortunately it was not possible to record filmed responses from within the Qualtrics platform. However, all participants were familiar with recording themselves during class exercises. The video responses were collected separately and linked to the test via an ID number presented to participants during the test. Video responses were reviewed and marked as valid or invalid corrections, after which this data was treated anonymously. 
To create the NZSL parts of the test items, a native Deaf NZSL user was filmed signing the sentences. In section 4.6.4.2 - Making sense of search results it was found that dictionary users sometimes assess the validity of a sign on the basis of their familiarity with the signer in the video. For this reason, a Deaf person was selected who was not a sign model in the ODNZSL and who had not been involved in teaching NZSL classes in the institutions from which test participants were recruited. One challenge in creating the NZSL parts of the test was that incorrect sentences had to be signed with the same fluency and apparent veracity as correct sentences.

A further challenge in creating the test instrument was that participants had to be able to look up individual signs used in the NZSL sentence, and to select individual signs to indicate errors. Since referring to signs by English glosses would be inappropriate here, still shots were taken of each sign used in the videos. Altogether, more than 300 still images were created and embedded in the test.

The complete test instrument is shown in Appendix B2. A sample of the test instrument with working videos and hyperlinks can be viewed online: http://vuw.qualtrics.com/SE/? SID=SV_bimcirveCqqiOP3. 
Comprehension E_1

a) Watch this NZSL video:

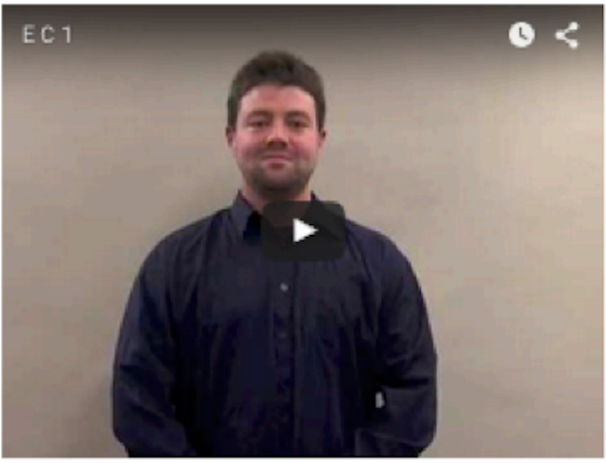

b) Use the dictionary:

Click on a still image of a sign from the sentence you have just watched. This will allow you to view information about this sign in the NZSL Online Dictionary.

First click on the image with a thick blue border. This is the target sign for this sentence.

You can then click on other images to look up dictionary information if you wish.
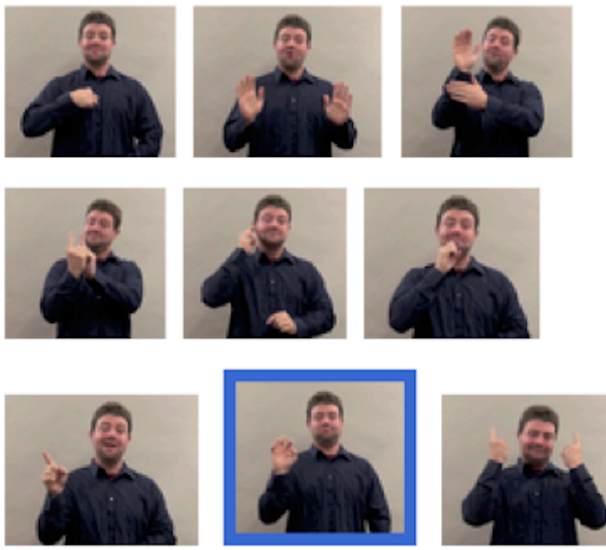

c) Is this translation correct or not?

While on holiday abroad I met a Deaf man. I couldn't understand his signing.

Tick CORRECT if you think this is an accurate translation of the NZSL sentence.

If you think there is an error, tick the box below the word(s) you think are incorrect, and type your correction.

\begin{tabular}{ccccc}
\hline & While on & a Deaf & couldn't his \\
CORRECT holiday abroad I met man. & understand signing.
\end{tabular}

Correction:

Figure 5.16: Format of a question in the comprehension block of the online test 
Production E_1

a) Target sentence:

You are trying to express the following sentence in NZSL:

He tried to pick up a girl at the bar. He bought her a drink.

The target word or phrase for this sentence is underlined and highlighted in blue.

b) Use the dictionary:

What search term(s) would you use to look up the target word or phrase in NZSL Online?

Click on the NZSL Online logo to carry out this search.

\section{NZSL}

ONL.INE

Are there any other parts of the sentence you would like to lock up in the dictionary?

Yes: I also want to look up these words or phrases:

No

c) Production

Watch this video which shows a version of the target sentence in NZSL:

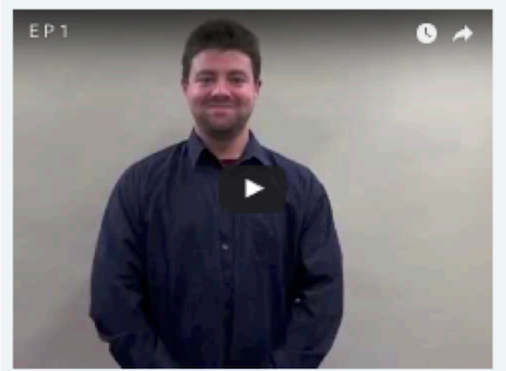

The target word or phrase is shown in this still image. Click on the image to view information about this sign in the Online NZSL Dictionary.

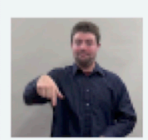

Tick CORRECT if you think this is an accurate translation of the English sentence. If you think there is an error, tick the box below the sign(s) you think are incorrect. (you
may have to scroll).

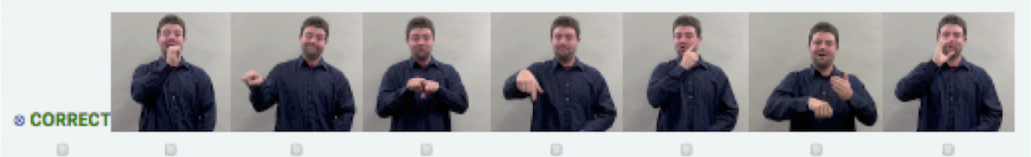

Record your correction on your lab computer and save it. Please sign your ID number and question number. You do not have to sign the full sentence - just the sign(s) you want to correct.

I have recorded and saved my correction.

Figure 5.17: Format of a question in the production block of the online test 


\subsubsection{Participants}

Participants were recruited from intermediate classes in NZSL at university (B.A.) level. Only two New Zealand universities offer intermediate and advanced study in NZSL:

- Victoria University of Wellington: NZSL Studies programme.

- Auckland University of Technology: NZSL and Deaf Studies, and NZSL-English Interpreting programmes.

Students taking second year NZSL papers (course modules) in any of these programmes will on average have completed 100-120 hours of formal NZSL learning, including both language classes and papers on Deaf community and culture.

The yearly intake of students is small: second year paper enrolments are usually between 1020 students. At the time of recruitment for the current study, 12 students were enrolled in the second year NZSL paper at Victoria University and 9 students at AUT University. A total of 21 participants was therefore available.

Since this small number of participants does not allow for a robust statistical approach, I considered recruiting more NZSL learners from other sources. However, while some community education classes in NZSL exist at intermediate levels, at the time of this study there was no consistent curriculum for these classes. Demographically, community education classes are more varied, with students of a wide range of ages, educational backgrounds, and familiarity with ICT. The location and facilities of community education classes would also make it difficult to administer the test. I therefore decided not to include community education classes in my sample and proceeded with the small number of 21 participants of a more consistent background.

\subsubsection{Procedure}

To increase the ecological validity of the online test, it was scheduled as part of a language lab, during which students would normally be expected to carry out computer- or videobased classroom activities. Four participants were unable to attend the lab or experienced technical difficulties during the lab. These participants completed the test independently at other times during the same week.

Participants were told that the test was part of their normal classroom activities, but that this exercise was not assessed and that the results would not be seen by their course teacher. The 
consent form, included in the online test, gave participants the choice not to submit their results.

Information on how to complete the test, presented on screen at the beginning of the test, included instructions for participants to complete the test independently without discussion with others, and to use the information in the dictionary as fully as possible.

To enable participants to complete the test within their normal class time, only two blocks (20 test items) were shown to each participant. The presentation of blocks was randomised but balanced so that each block would receive a roughly equal number of responses. The 10 reception questions were presented first, followed by the 10 production questions. The total time taken to complete the test varied greatly, from 31 minutes to 100 minutes. The average length of time taken was just over an hour ( 1:01:46).

\subsection{Results and discussion}

\subsubsection{Quantitative analysis}

To account for the variable number of responses to each question, each test item was given a score by calculating the mean number of correct responses for that item. A 'correct response' was defined as either identifying an errorless sentence as correct, or identifying the error.

The mean score per item was $71 \%$ (range $13-100$, SD 24). A summary of the results is presented in table 5.8 .

\begin{tabular}{ll}
\hline Mean score (\%) & Number of test items $(\mathbf{n}=\mathbf{5 0})$ \\
\hline $0-25$ & 5 \\
$25-50$ & 5 \\
$50-75$ & 15 \\
$75-100$ & 25 \\
\hline
\end{tabular}

Table 5.8: Number of test items per mean score of correct responses

There were a total of 40 items with a mean score of $50 \%$ or over. This indicates that these 40 items (or $80 \%$ of the total test items) were answered correctly by at least half of the participants.

Of the 25 items with mean scores of $75 \%$ or more, seven items achieved a score of $100 \%$, i.e. they were answered correctly by all participants. These seven items will be considered 
unproblematic in that the current dictionary content is sufficient for the majority of intermediate students to identify their meaning and correct usage.

To find out which factors might influence item scores, paired t-tests and a one-way analysis of variance (ANOVA) were carried out.

\subsubsection{Error vs. no error}

There was a significant difference between scores for items containing an error $(\mathrm{n}=15, \mathrm{M}=$ 64.17\%; $\mathrm{SD}=22.71$ ) as compared to items without an error ( $\mathrm{n}=35, \mathrm{M}=87.81 \%$; $\mathrm{SD}=16.90)$; $\mathrm{t}$ $(48)=-3.61 ; p=.0007$ (2-tailed). This shows that items without an error were more likely to be answered correctly.

Out of the 15 test items without an error, 6 were marked as correct by all participants. Only one error-free item received a mean score of less than $50 \%$ (i.e. more than half of the participants identified some part of the test sentence as incorrect even though it was correct).

\subsubsection{Comprehension vs. production}

Although comprehension items $(\mathrm{M}=76.88 \%)$ scored higher than production items $(\mathrm{M}=$ $65.50 \%$ ), the difference was not significant: $\mathrm{t}(48)=1.74 ; \mathrm{p}=.09$ (2-tailed). Nevertheless, given the fact that the highest mean score was achieved for the 5 questions in comprehension block AC $(M=91.20 \%)$ and the lowest mean score for production blocks BP and CP $(M=55.20 \%$ and $\mathrm{M}=55.40 \%$ respectively), in further analysis comprehension and production items were separated.

\subsubsection{Test item category}

A one-way analysis of variance (ANOVA) was carried out to find out whether the category of the test item influenced the item score. Results are presented in table 5.9.

\begin{tabular}{lllllll}
\hline Category & & $\begin{array}{l}\text { Sum of } \\
\text { squares }\end{array}$ & df & $\begin{array}{l}\text { Mean } \\
\text { square }\end{array}$ & F. & P \\
\hline Comprehension & $\begin{array}{l}\text { - Culture-bound } \\
\text { - Idiomatic } \\
\text { - Polysemous }\end{array}$ & 0.059264 & 2 & 0.029632 & 0.84 & 0.45 \\
Production & - Metaphoric/metonymic & 0.031497 & 2 & 0.015748 & 0.2 & 0.82 \\
& $\begin{array}{l}\text { - Vocabulary type/word class } \\
\text { - Other }\end{array}$ & & & & & \\
& & & & & & \\
\hline
\end{tabular}

Table 5.9: Analysis of Variance of test item categories 
None of the categories approached significance, showing that items in any category were as likely to be problematic as items in other categories.

When the error-free items were removed from the analysis, results still indicated no significant differences between the categories for either comprehension items $(\mathrm{F}=1.19 ; \mathrm{p}=$ o.33) or production items $(\mathrm{F}=0.1 ; \mathrm{p}=0.91)$.

Multiple regression analysis also showed that no categories were a good predictor of the mean item score.

\subsubsection{Further analysis}

Since the initial statistical analysis did not provide a clear answer to the research question, a qualitative approach was taken next by examining common factors in incorrect responses. There were 10 test items for which the mean score fell below 50\%, i.e. for which half of more learners were not able to identify the error, and which could therefore be considered to be in need of further dictionary information. These test items are listed in table 5.10.

This list confirms that there was at least one difficult test item in each category. Although the majority of low scores occurred on the production side of the test, learners also experienced difficulties with comprehension. In the case of test item $\mathrm{CP}_{5}$ HEARING, for example, this sign was not recognised as referring to people in terms of their relationship with the Deaf community, rather than to the physical sense of hearing something.

\begin{tabular}{lll}
\hline Test item no. & English main gloss(es) & Category \\
\hline AP2 & discard, leave & vocabulary type / word class \\
BP1 & exasperated with & metaphoric / metonymic \\
BP2 & dysuria & vocabulary type / word class \\
BP5 & Deaf & other \\
CC1 & ask, remind, tell & culture-bound \\
CC4 & silly me & idiomatic \\
CP2 & keep in mind & vocabulary type / word class \\
CP5 & hearing & other \\
DC5 & centre, Central, middle & polysemous \\
DP5 & decrease & other \\
\hline
\end{tabular}

Table 5.10: Test items with a mean score of $50 \%$ or less 
Learners also had difficulties with test items that required more subtle sense distinctions. For example, to answer test item $\mathrm{CC}_{1}$, learners had to make the distinction between the literal action of tapping someone's shoulder to gain their attention (a cultural behaviour within the Deaf community), and its abstracted sense of asking, telling or reminding someone. The sense distinction between 'middle' and 'Central (zone)' in test item DC 5 may have required general (non-linguistic) knowledge of the zones into which New Zealand is divided for (Deaf) sporting events.

Learners were not able to identify the different collocation patterns of two signs used in test item DP5 DECREASE. The collocational error in test item BP1 EXASPERATED-WITH was also not recognised. It seems that the iconic or metaphorical characteristics of these signs (the direction of movement in the case of DECREASE, and the metaphor of 'wringing someone's neck' in the case of EXASPERATED-WITH) were not helpful.

When sign phrases were used as headwords but their English equivalents were single words, the usage patterns of the individual signs were not apparent to learners. In test item $\mathrm{BP}_{2}$, the technical term DYSURIA therefore obscured the fact that the sign headword is a phrase consisting of the signs HURT and PEE-(MALE) and can therefore not be applied to a female subject. Similarly, the fact that test item $\mathrm{BP}_{5}$ DEAF, a phrase consisting of signs FULLY and DEAF (see section Distinguishing senses under section 5.3.1.1), cannot be used in collocations such as 'Deaf sports' or 'Deaf club' was perhaps difficult to identify because the English main gloss is simply 'Deaf'.

In both these cases, usage notes in the dictionary entry provide relevant information that would avoid these errors. The fact that more than half of the participants did not identify the error indicates that, in spite of the instructions to use dictionary information fully, the usage notes were not read. The support provided by examples showing correct collocation patterns was also not used. For test item $\mathrm{DC}_{5}$, the reference to 'NZ Deaf games' in the example may have been distracting (see section 5.3.1.3 - Cultural support), whereas for test item $\mathrm{DP}_{5}$, examples of correct collocations may have been ignored because there is no indication that the collocations illustrated are exclusive.

In test item CP2 KEEP-IN-MIND, the information in the dictionary was misleading. Both the video showing the production of this sign and the example include a facial expression that signals a negative connotation, creating a sense that is closer to 'bearing a grudge' rather than a neutral 'bearing something in mind'. Learners assumed from the dictionary information 
that this facial expression is an integral part of the sign even when it is used in a positive context (keeping in mind a possible birthday gift).

The low scores of the two remaining items may have been due to the construction of the test sentence. For AP2 DISCARD / LEAVE, the distractor sign GIVE was substituted for the intended sense 'give away', which is a secondary gloss for the test item. When learners marked the sentence as correct, this therefore only indicates that they were not able to use the distractor sign correctly, but it does not give a direct indication about their knowledge of the target sign. The English translation of the sentence in test item CC4 SILLY ME used the secondary gloss 'oops' at the beginning of the sentence, rather than in the equivalent place that the sign SILLY ME was used in the signed sentence. This led to learners identifying an error when the translation was in fact correct.

This examination of the lowest scoring items has shown that learners experienced difficulties with some test items, as was predicted from the evaluation of dictionary information in this chapter and from dictionary user behaviour patterns in the previous chapter. However, it should be borne in mind that most of the test items (80\%) were answered correctly by more than half of the participants. The conclusion might be drawn, then, that the information provided by the ODNZSL is generally sufficient for the majority of learners. To confirm the extent to which learners' needs are indeed met by the current information, two further questions should be asked. Firstly, how did individual learners perform on the test? And secondly, were learners also able to correct the errors they identified?

\subsubsection{Participants' scores}

Participants' scores on the 20 test items ranged from $40 \%$ to $100 \%$, with a mean score of $68.6 \%$ (SD 17.5). Even given the small sample size, these scores suggest that there was no undue influence of a few very high or very low participant scores on the results per test item.

A further factor to consider was whether time spent on the test influenced participants' results. Duration of the test showed no direct correlation with participants' scores. The four highest scoring participants were amongst those participants who took the longest time to complete (57 minutes and over); however, two of the four lowest scoring participants took similar times to complete (54 minutes and over). Conversely, two high scoring participants with a score of $80 \%$ took only 29 and 31 minutes respectively to complete the tests. Short completion times suggest that some participants did not examine the dictionary information in great detail before answering questions. 
These findings have implications for the validity of the test instrument as a measure of the influence of dictionary information on NZSL learners. These implications will be further discussed in section 7.3 - Testing the effectiveness of dictionary definitions when I will report on the results of the repeated test to measure the effect of signed definitions.

\subsubsection{Error correction}

The statistical analysis showed that it was easier for learners to confirm that a test item was used correctly in a sentence than it was to identify incorrect usage. This confirms that some language tasks are easier than others. To correct errors (in either language direction) requires much more specific knowledge about the precise meaning and use of a sign than is required to only identify the error.

When appropriate error correction was included in the analysis, the mean score per item dropped from $71 \%$ to $65 \%$ (range 13-100, SD 25), and the number of items that were answered correctly by at least half of the students dropped from $80 \%$ to $68 \%$. Table 5.11 lists test items for which the correction score was lower than the error identification score.

Examining the nature of the corrections for these test items sheds further light on the kind of difficulties that NZSL learners experienced. As was the case for some test items with low scores, some mistakes in error correction were due to the test format. In two cases (test items $\mathrm{EP}_{1}$ and $\mathrm{EP}_{5}$ ), a participant did not record their video response. Two further responses suggest that the participant misunderstood the task. In the sentence for test item $\mathrm{BC}_{2}$ UNINTELLIGIBLE-VOICE, the target sign was incorrectly translated as 'accent'. Instead of correcting the English translation, the participant described another sign that translates as 'accent'. A similar response occurred for test items BC 5 SMALL-ROUND-FOOD-PLURAL and CC 3 ONE-UP.

In several cases, the participant identified several signs as incorrect, including the target sign, but then proceeded to provide a correction only for a non-target sign: e.g. providing an alternative for the sign LIVE in the sentence for item AP4 TIME SPAN, for the sign TELL in the sentence for item BP2 DYSURIA, for the sign AUDIENCE in the sentence for item $\mathrm{CP}_{5}$ HEARING, and for the sign GIVE in the sentence for item EP2 SPEECH. 


\begin{tabular}{|c|c|c|c|c|}
\hline $\begin{array}{l}\text { Test Item } \\
\text { no. }\end{array}$ & English main gloss & Category & $\begin{array}{l}\text { Mean error } \\
\text { identification } \\
(\%)\end{array}$ & $\begin{array}{l}\text { Mean } \\
\text { correction (\%) }\end{array}$ \\
\hline AC4 & keep inside oneself & idiomatic & 88.9 & 44.4 \\
\hline AP3 & gesture & vocabulary type & 77.8 & 66.7 \\
\hline AP4 & time span & other & 77.8 & 44.4 \\
\hline $\mathrm{BC} 2$ & unintelligible voice & culture bound & 62.5 & 50.0 \\
\hline BC5 & (small round) food-plural & polysemous & 75.0 & 37.5 \\
\hline BP2 & dysuria & vocabulary type & 25.0 & 12.5 \\
\hline CC3 & one-up & idiomatic & 87.5 & 62.5 \\
\hline CP5 & hearing & other & 50.0 & 37.5 \\
\hline DC2 & hard of hearing & culture bound & 75.0 & 37.5 \\
\hline DC3 & been to, have experience of & idiomatic & 75.0 & 62.5 \\
\hline EC1 & one-handed fingerspelling & culture bound & 66.7 & 55.6 \\
\hline EC3 & oh good & idiomatic & 88.9 & 77.8 \\
\hline EP1 & hook up & metaphoric & 77.8 & 66.7 \\
\hline EP2 & speech & vocabulary type & 77.8 & 66.7 \\
\hline EP5 & then & other & 55.6 & 44.4 \\
\hline
\end{tabular}

Table 5.11: Test items with a lower mean score for correction than for error identification

In the comprehension section, one participant provided an alternative translation of the sign shown in figure 5.18, indicating that it should be translated as 'land' instead of 'beach'.

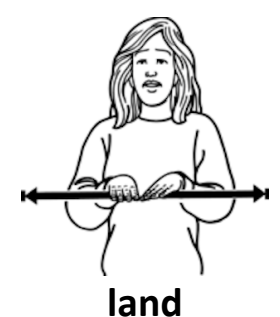

whenua, pito whenua beach, country, farm, field, garden, grass, ground, lawn, park, playground, property

Figure 5.18: A polycomponential, partly specified sign in NZSL

Although this response did not indicate whether the target sign DC3 BEEN-TO / HAVEEXPERIENCE-OF had been understood, it showed that the polysemy of polycomponential, partially specified signs is difficult for some learners, when several translational equivalents are plausible in the context (such as 'beach' or 'land'). These difficulties were also evident in test item $\mathrm{BC}_{5}$ SMALL-ROUND-FOOD-PLURAL, where responses varied between two plausible 
translational equivalents, 'beans' and 'peas'. One participant opted for the response 'small round things e.g. berries', even though the translational equivalent 'berries' was less likely in the sentence context of food being served with meat at a restaurant. The other test item of this nature asked participants to distinguish between the senses 'dirty' and 'greasy' of a sign that can also have the senses 'fine / delicate', 'fabric', 'thing / stuff / substance', 'sand / soil', and 'texture'. This item had a mean score of $66.7 \%$. Overall, these responses indicate that the precise reference of polycomponential signs is not always resolved by the context.

Responses for two items in the culture bound category (DC2 HARD-OF-HEARING and EC1 ONEHANDED-FINGERSPELLING) and one item which also relates to Deaf culture (AP3 GESTURE) showed that although participants had some familiarity with the semantic and cultural content of these signs, they were not always able to reflect nuances in usage of these signs or their translations in their own production. For example, one response used the informal TALK-INSIGN-LANGUAGE (see table 5.2) as a correction instead of the more general SIGN. The sign HARDOF-HEARING is mostly used as a label for a person with a hearing loss who is not a member of the Deaf community; but one participant expressed uncertainty about using the phrase 'hard of hearing' in English and instead chose 'has difficulty hearing'. Responses to test item EC1 included 'one-handed signing', although this is a minor distinction given that in some contexts, this sign can extend its strict sense of 'one-handed fingerspelling' to 'a stretch of discourse in a foreign language that includes a large amount of fingerspelling'. Another response simply stated 'fingerspelling', although the use of one hand is relevant here since the two-handed alphabet used in New Zealand is referred to by a different sign.

The grammatical structure of the NZSL sentence, rather than the semantic content of the target sign, was the cause of incorrect answers for test item $\mathrm{AC}_{4}$ KEEP-INSIDE-ONESELF. This test item is shown in figure 5.19.

AC4 KEEP-INSIDE-ONESELF

Test item video

Test translation (error marked in bold):

My friend was having a barbecue. He didn't tell me because I had a sore stomach.

Correct translation (correction marked in bold):

My friend was having a barbecue. He didn't tell me. I felt hurt but I kept it to myself.

Figure 5.19: Test item $\mathrm{AC}_{4}$ KEEP-INSIDE-ONESELF 
The figurative sense of feeling emotionally hurt but keeping it to yourself was identified correctly by all but one respondent. However, in 5 responses the action of 'keeping it to yourself" was wrongly attributed to 'my friend'. The NZSL sentence in this test item is an example of a grammatical structure where no overt subject is included (pro-drop); a structure that is common in NZSL (see also section 5.3.1.2 - Grammatical support) and that can lead to ambiguities that are difficult for learners to resolve.

\subsection{Summary of findings}

The results of the test indicate that in most cases, dictionary information in the ODNZSL is sufficient for intermediate learners to identify correct and incorrect uses and senses of signs. A small number of test items were problematic and learners also experienced more difficulties correcting the errors they had identified. None of the test categories were good predictors of difficulties, nor was the distinction between comprehension and production tasks. A qualitative examination identified a number of problems, which can be summarised under four factors:

1. Linguistic factors

- collocations;

- partially specified polycomponential signs;

- non-manual features;

- nuances in culture-specific terms / usages;

- larger grammatical structures (e.g. pro-drop);

- lack of awareness of multiple sign variants (signalled by learners replacing correct signs with better known variants);

- metaphor and iconicity did not have an influence on learners' sign production and comprehension.

\section{Dictionary factors}

- inadvertent connotations in the example were misleading;

- the pairing of sign phrases as headwords with a single word English gloss obscures the grammatical behaviour of the sign phrase; 
- even when examples provide useful collocation or usage information, learners did not make use of this information, perhaps because it is unclear whether the examples exhaustively covered all possible collocations, or because the example contained a distracting cultural reference.

3. User factors

- usage notes and possibly examples were not noticed by some participants;

- the short test completion time of some high scoring participants suggests that these participants achieved these scores without using the dictionary.

\section{4. $\quad$ Test factors}

- error-free test items were easier than items with an error;

- Some participants misunderstood the comprehension and production tasks or failed to record their response;

- The construction of some test items meant that the participants' understanding of the relevant features of the target sign could not be assessed from their responses.

\subsection{Limitations}

The main limitation of this study was the low number of participants and (anecdotally) the wide range of actual NZSL proficiency within these second year classes, with some students having had considerably more opportunities to use NZSL outside of the classroom than others. It would therefore be inadvisable to draw any strong conclusions from the statistical analysis presented here. The overlap between some test categories and the fact that some test items could be placed in multiple categories may have further weakened the analysis. However, the indicative results of the quantitative analysis were useful as a guide to determine which test items warranted further qualitative investigation.

As indicated in the reported results, some test items did not measure participants' ability to understand or use the target sign, but instead gave information about a distractor item or assessed a different issue than the category would suggest. A pilot study would have prevented these issues; however, this would have further reduced the potential participant group for the actual test. 
A further limitation is that the highly labour-intensive test preparation, including videos, still images and hyperlinks for each test item, may make this kind of test format impractical as a regular evaluation tool.

Finally, the ecological validity of the test was affected by the instruction to use dictionary information fully in all cases, regardless of the participant's familiarity with the test item.

\subsection{Conclusion}

The overview of the role of dictionaries in language learning presented in this chapter confirmed that definitions potentially contribute to decoding, encoding and vocabulary learning needs, but that they are only likely to be effective in combination with other dictionary information and when they are presented in a manner that can be understood by language learners.

Hybrid dictionary formats with a mixture of definitions, examples, and equivalents, especially in electronic form, were found in the literature to be of benefit to a wide range of language learners. This finding provided support for the inclusion of signed definitions in sign language dictionaries such as the ODNZSL to supplement their current content. However, the literature also showed that language learners do not access definitions equally for all lexical items. This suggested that a study was needed to identify where the current information in the ODNZSL does not adequately meet the needs of language learners.

An examination of the two main elements in current ODNZSL entries-equivalents or glosses, and examples of use-showed that in spite of a systematic approach towards selecting both translational and explanatory equivalents, the information provided by these equivalents was in some cases unhelpful or misleading. Examples in the ODNZSL were shown to serve a number of functions but to be less successful when learners relied on a single example for information on both meaning and grammatical usage simultaneously. Multiple examples targeted at decoding and encoding needs separately were recommended in the literature. Signed definitions could serve as a particular meaning-focused example in this context and could also provide explicit information about the shared experiences and values of the Deaf community without the distraction that this cultural information potentially causes when presented implicitly in examples of use.

The review of the current content of the ODNZSL identified categories of potentially problematic lexical items. These categories were tested with intermediate learners. A 
statistical analysis showed that in spite of potential lack of equivalence or insufficient dictionary information, the majority of test items were not difficult for most learners. The statistical analysis did not identify any category as being more problematic than others.

While test results had limited statistical significance, a qualitative review of learners' responses showed that for the small number of problematic test items, there were several linguistic factors that were difficult for learners. Current dictionary information was not sufficient especially in the case of sign phrases and to help learners identify restricted collocations. Some participants were also found not to make use of dictionary information, in particular usage notes. This finding confirms results of the user study referred to in section 4.9 - Conclusion and suggests again that customisation of dictionary information for different users and providing training in dictionary use for learners will be crucial factors determining whether signed definitions will be effective.

In spite of the limitations of the test, the results suggest that there are indeed a small number of sign lemmas from a range of categories for which additional information in the dictionary is required. The next two chapters will investigate whether signed definitions, in particular folk definitions, are able to provide this information. Chapter 6 - Folk definitions will describe the elicitation of folk definitions for 15 of the low-scoring test items identified as problematic in the current test. Chapter 7 - From folk definition to dictionary definition will examine the suitability of folk definitions as part of the dictionary entry. This chapter will include a repeat of the current test with a matched group of intermediate learners to evaluate the effect of including signed definitions. 


\section{Chapter 6: Folk definitions}

\section{Introduction}

So far in this thesis I have addressed the question whether signed definitions can make a contribution to sign language dictionaries by examining the format, users, and content of the ODNZSL. In this chapter the focus turns to the form and content of the definitions themselves.

I will argue that so-called 'folk definitions' - spontaneous lay explanations of word meaning by native speakers - are a useful basis for developing signed definitions. The chapter begins with an overview of the literature, providing several perspectives on folk definitions to support this claim.

- I will first examine the function of folk definitions as data. A small number of anthropologists and linguists have employed folk definitions as a fieldwork tool in documenting languages, especially oral languages. Dingemanse (2015) has made a convincing case for reviving this method of gathering rich data while postponing the translation stage of language documentation. Sign languages, as unwritten languages for which language description and documentation are often still at an early stage (see Chapter 2 - Sign languages and the sign language lexicon and Chapter 3 - Sign language lexicography), could benefit from this approach to data gathering.

- Next, I will describe the role of spoken definitions and explanations in language learning. Informal explanations of word meaning are common both in parent-child interaction and in classroom discourse. Some research has suggested that characteristics of such explanations enhance language learning and vocabulary retention.

- I will then focus on folk definitions in dictionaries. I will outline reasons why some lexicographers have recommended a move away from traditional dictionary definition formats, in favour of more informal explanations. The full sentence definition format in the Collins COBUILD English Language Dictionary (Sinclair, 1987 a) has been the most systematic application of folk definitions in dictionaries to date. The applicability of fullsentence definitions to the proposed signed definitions will be discussed in the light of criticisms of this definition format, and differences between the written and spoken/ signed medium. 
Having established a rationale for choosing a folk definition format, I will go on to describe how the format of explanations by Deaf people is influenced by the different contexts in which signed explanations of sign / word meaning have been found to occur, including both face-to-face discourse settings and in video form in dictionaries and language learning resources.

After considering further methodological issues in gathering folk definitions, I will report on a study in which folk definitions were elicited from 13 Deaf NZSL users, using the 15 signs that were found to be problematic for learners in the previous chapter (see section 5.8 - Summary of findings). I will discuss the semantic content of the elicited folk definitions; other content such as contextual information about register, variation, grammatical features and cultural connotations; and selected aspects of their form. The aim of this analysis is to identify common features that could form the basis of dictionary definitions. I will draw particular attention to those areas where signed folk definitions may differ from folk definitions in spoken languages, since these areas have implications for the final format of signed dictionary definitions.

\subsection{Folk definitions as data}

Folk definitions, defined by Dingemanse (2015) as "native, informal, multi-modal explanations of linguistic items", were first used as data on word meaning by researchers carrying out linguistic fieldwork in the anthropological tradition. There are a number of parallels between such fieldwork and sign language documentation and lexicography. Firstly, linguistic anthropological research is often initiated by researchers from outside of the language community, as has been the case for much sign language research. Secondly, the languages being documented in linguistic anthropology have frequently been unwritten languages used in smaller communities, and although there are factors that make sign languages especially challenging to document in written form, methods developed to gather data specifically in oral communities are likely to be useful models. Thirdly, as outsiders, anthropological researchers have to acquire sufficient fluency in the language to be able to observe, understand, and participate in the community and as a first step in their research. They therefore often create a list of vocabulary with glossed equivalents in their own first language. These word lists may become the first bilingual glossaries or dictionaries of the language being documented. In section 3.1 - History and purposes of sign language dictionaries it was noted that many sign language dictionaries were similarly motivated by 
outsiders' immediate communication needs. Given these parallels between language documentation and lexicography in anthropological fieldwork and in sign language research, it is reasonable to assume that the arguments for using folk definitions as data apply equally to both.

Folk definitions were first investigated by researchers who recognised the inadequacies of the bilingual glossary method. Although helpful as a vocabulary learning tool for non-native researchers, the lack of equivalence between two words in different languages, and the tendency of glosses to obscure culture-and language-specific characteristics of the listed vocabulary, were already noted in previous chapters (see sections 3.2.1 - Representing signs without a written form and 5.2.1 - Degrees of equivalence). The 'word list' approach to language documentation was also found to be subject to the problem of 'indeterminacy of translation' (Quine, 1960): when a word is taken out of the context in which it was used, its reference and the part it plays in determining the meaning of longer utterances are uncertain and may be misrepresented by the allocated gloss. Several authors therefore proposed that vocabulary should first be described where possible in native speakers' own words, and in authentic contexts.

Motivated by Weinreich's (1967 / 1970) argument that semantic description could start by making explicit native speakers' intuitions about relationships between words and concepts in their language, Casagrande and Hale (1967) collected around 8oo folk definitions from a native speaker of Papago. Although their original intention was to use these folk definitions as prompts for an elicitation exercise with other native speakers to research lexical variation, Casagrande and Hale found that the folk definitions themselves provided valuable data about the semantic and grammatical structure of the language.

Mosel (2011) noted the benefits of recording and analysing folk definitions in dictionaries of endangered languages. Since most dictionary projects for endangered languages are small and led by researchers from outside of the speech community, it is important that these first dictionaries represent native speakers' interpretations of the meaning of the dictionary headwords and their relationship with other expressions in the language. This argument has also been made in relation to sign language dictionaries by Brien and Turner (1994) and Brien and Collins (1997). Having folk definitions available in a dictionary enables further linguistic and anthropological research, and unlike bilingual word lists, folk definitions can later be used for the development of monolingual resources and teaching materials. 
Mosel recognised that in most cases, dictionary projects have to be completed before large enough linguistic corpora can be established. Elicited data, including folk definitions, will therefore remain important for lexicography in smaller language communities, especially for non-literate societies. In section 3.4.9 - Corpora and corpus-based lexicography, I commented on a similar situation for sign language research.

Dingemanse (2015) argued that folk definitions call on natural explanatory routines that are near to native language users' experiences. This has the benefit of significantly widening the number of people from whom data can be gathered, since no expert knowledge is required beyond native language competence. The informal nature of folk definitions also ensures that they reflect typical face-to-face interactions. Considering the small number of 'native' Deaf sign language users described in section 2.1.3 - Threats to the vitality of sign languages, increasing potential sources of data would be of great benefit to sign language research.

Dingemanse's own study of folk definitions involved recording folk definitions on video to examine the multimodal nature of explanations about ideophones: words that have an iconic form-meaning relationship through sound symbolism. Dingemanse's use of multimodal data, and his observation that folk definitions offer a way of studying word categories for which the meaning is hard to elicit by traditional methods, provide a further rationale for applying the folk definition method to sign language research.

\subsection{Spoken definitions and explanations}

In addition to their role as data on language use, folk definitions have also been studied in the context of language learning.

With regard to first language acquisition, research has concentrated on children's development of definitional skills as an indication of the vocabulary they have acquired and their recognition of the symbolic nature of words. A longitudinal study (Kurland \& Snow, 1997) found that the quality of children's definitions changed over time, with definitions becoming more formal in structure as the children progressed. Formal definitions in (Aristotelian) philosophy have been said to consist of a term, a class or category, and one or more characteristics or attributes that differentiate the term from other terms in the same class (e.g. Flowerdew, 1992; Atkins \& Rundell, 2008). This kind of analytical definition has been taken as the foundation for most traditional dictionary definitions (Hanks, 1987; Stock, 1988; Atkins \& Rundell, 2008) and there is evidence that the use of formal definitions in 
spoken language has strong links with literacy development (Sinclair, 1986) and schooling, including practice at defining and teachers' classroom discourse modelling decontextualised language (Kurland \& Snow, 1997). Kurland and Snow found that when they repeated the defining task in their study with the child participants' mothers (who had had limited formal schooling after the compulsory education age), longer, narrative-like, rambling explanations which lacked one or more elements of the formal definition were the norm.

The hypothesis that a person's lexicon development and productive definition skills are influenced by the type of definitions that they are exposed to has also been investigated in the context of second language learning. Chaudron (1982) analysed high school and university subject teachers' elaborations about the meaning of vocabulary in their speech addressed to EFL learners. 'Elaboration' is a broader concept than 'definition': Chaudron labelled as 'elaborations' any instances where a teacher "in some way defined, qualified, questioned, repeated, paraphrased, exemplified, or expanded upon" a term or expression. (p. 171). Within this broader set of strategies, Chaudron identified phonological, morphological, syntactic, semantic and wider discourse features that were characteristic of teachers' deliberate and implicit attempts at clarifying word meaning. Such features included clearer or slower pronunciation, extra stress, and particular intonation patterns; the use of similar morphological structures for the term being defined and any synonyms or explaining terms; the use of repetition, sentence conjunction, substitution and ellipsis; and frequent use of deixis. Not all strategies were equally effective. For the present thesis, the use of these wider phonological, morphological, syntactic and discourse strategies in spoken explanations is relevant since the final form of signed dictionary definitions is still a 'live' performance on video that can only partly be scripted and therefore may include strategies that would not be found in written, depersonalised definitions.

Chaudron also analysed the semantic and cognitive relationships between terms being defined and the teachers' elaborations. He identified three different categories:

- subordinate: the elaboration is a specific instance of the term being defined. This included showing, pointing at, or drawing real-life objects or images, or giving verbal examples focusing on the function of the item or a context in which it would be used;

- equivalent: the elaboration is a synonym, antonym, near-synonym or comparison of the term being defined. In situations where the students had the same language background, Chaudron noted that teachers might provide an equivalent in the students' L1; 
- superordinate: the elaboration places the term being defined into a higher class, i.e. a hypernym is provided. Naming (this is [called] an X) and defining (this is a [kind of] X [which / who] ... ) are common superordinate strategies.

This classification shows that formal definitions, providing both a superordinate term and identifying features, are only one of a range of explanation strategies found in spoken language, even in an academic context.

Stock's (1988) list of features of folk definitions when compared with traditional dictionary definitions overlaps in part with Chaudron's findings: for example, the frequent use of direct demonstration, repetition, use of subordinate examples, and use of synonyms. She also noted that functional definitions were more frequent than concentrating on the visual aspects of referents, and that folk definitions often embedded information about register and context. Stock argued that these features could be helpful for language learners and, by extension, as a model for dictionary definitions:

"In my own experience lexicographic definitions, however elegant and logically constructed (indeed particularly when elegant and logically constructed) can be unhelpful as an aid to learning new meanings. (...) the immediacy, the accessibility and the vividness of folk definitions often make them more memorable and consequently more likely to be of help in both decoding and encoding."

(pp. 86-87)

Richards and Taylor (1992) also argued that folk definitions are a more useful model than formal dictionary definitions for L2 learners. They found that the use of particular defining strategies depended on two factors: firstly, the kind of word that was being defined; and secondly, the linguistic proficiency of the person providing the definition. Richards and Taylor noted that formal analytic definitions were only used for about $50 \%$ of nouns in their data and hardly at all for other word categories. L2 learners were more likely than native speakers to use a definition based on characteristics rather than using a superordinate, and Richards and Taylor suggested that intermediate language learners may not yet have developed the range of classifying terms required to use more analytic definitions.

Flowerdew's (1992) study of definitions in science lectures focused on spoken explanations as speech acts within a wider academic discourse genre. The large variation in form of spoken definitions led Flowerdew to conclude that it is important to describe typical definitional patterns, including syntactic and lexical 'signals' that were used to introduce definitions, so that learners are able to identify and eventually use these patterns themselves. 
Finally, Nation (2001) provided an overview of studies that found a positive effect on vocabulary learning from spoken explanations in context. Nation attributed this effect to the simple, direct nature of spoken explanations and found potential benefits of defining as a form of decontextualisation, i.e. focusing attention on the word itself rather than the role it played as part of a wider message. Like Chaudron, Nation argued that equivalents in the learners' first language were a simple but effective kind of definition.

In summary, research on the role of definition and explanation in language learning supports the use of informal, spoken folk definitions as a potential model for signed definitions. Spoken folk definitions were found to be beneficial to L2 learners' decoding, encoding and vocabulary learning. Examinations of the explanation strategies used by native speakers have found that although there are is a large variation in form, typical semantic categories and syntactic and lexical 'signals' could be identified that were used consistently to define particular word categories.

\subsection{Folk definitions in dictionaries}

Folk definitions have been considered by a number of lexicographers as an alternative to traditional dictionary definitions. Historically, lexicographers have based dictionary definitions on formal models in which words are assumed to have discrete senses that can be pinpointed precisely (see e.g. Zgusta, 1971). Three principles of traditional definitions have their basis in these semantic and logical models:

1. The genus and differentiae approach: the genus (a hypernym) places the word in a hierarchical class, and one or more differentiae specify what makes the word different from other words in the same class.

2. Necessary and sufficient conditions: words are defined by naming the conditions that have to be true for the word to be applied.

3. Substitutability: the definition can take the place of the word itself in a sentence without altering the truth or grammaticality of the statement.

Critical evaluations such as Stock (1988) have pointed out that these three principles of an ideal formal definition are rarely attainable in practice. For example, only certain words such as concrete nouns (and some verbs, according to Atkins and Rundell, 2008) fit into a taxonomy and can therefore be described successfully through the genus and differentiae approach. Attempts to list all the necessary criteria covering all instances of a word are also 
not realistic, and Stock showed that traditional definitions are in most cases too lengthy and technical to be substitutable in a real text.

Stock proposed that folk definitions offered a range of techniques that could usefully be applied to dictionary definitions. First of all, Stock noted the benefits of the short, simple and memorable wording of many folk definitions. Secondly, she argued that the tendency of folk definitions to explain the function of objects rather than providing a physical description would be more useful to dictionary users. Thirdly, the form in which traditional dictionary definitions are expressed often includes abbreviations and formulaic sentence structures that may be difficult to understand. Folk definitions, by contrast, are expressed in natural syntax and offer examples of the word in use. Finally, Stock found that folk definitions show register as an integral part of a word's meaning, offering more flexibility than treating register separately through a limited set of abbreviations.

Wider trends in lexicographical practice have lent further support to alternative definition styles that share common features with folk definitions. An increased focus on the needs of dictionary users meant that definitions began to be evaluated by how well they fulfilled their function as aids in decoding, encoding, and vocabulary learning for particular user groups, especially children and foreign language learners. Both the content and the form of traditional dictionary definitions were found to be frequently inadequate, being difficult to understand and at times misleading.

McKeown (1993) focused on vocabulary learning by children and found that traditional dictionary definitions frequently exhibited problems such as being too broad, using terms in the definition that were likely to be misinterpreted by young learners, using vague language, and spreading the definition over a set of disjointed components (for example, a set of nearsynonyms). McKeown argued that definitions should be created from a cognitive perspective instead, reflecting how humans think about and categorise objects. Research by Rosch (1973) had concluded that people based their judgments of category membership on a comparison with a typical example of the category. Rosch (1978) argued that categories are not completely distinct, but that some members will be recognised as more central, while there may be doubts about peripheral members that meet some criteria but not others. In this view, attempts to list all the necessary conditions for category membership are likely to fail, since there will usually be unclear borderline cases. Instead our attention should be focused on the clear cases, which Rosch labelled prototypes. Rosch's prototype theory gained currency in 
cognitive linguistics (e.g. Aitchison, 1987) and alongside other gradual developments in linguistic philosophy (see Hanks, 2015 for an overview), the understanding of word meaning as 'fuzzy' became more widespread in linguistics. The implications for definitions are that they should clearly describe the most typical features of a word.

Hanks $(1987,2015)$ furthermore emphasised that words do not have pre-determined senses independent of the context in which they are used. Rather, words have meaning potential that is realised in particular instances in which the word is used. Definitions should therefore help users to identify this meaning potential by focusing on the most likely sense(s) in which a word is typically used. Sinclair's COBUILD project, of which Hanks was a member, pioneered the analysis of large digital corpora to determine the most frequent word senses. The Collins COBUILD English Language Dictionary (Sinclair, 1987 a) was also the first dictionary to introduce a definition format based on the informal explanations of lay people. Although this format has sometimes been referred to as 'folk definition', Hanks (1987) and Sinclair (1987 b) used the term 'full-sentence definition'.

The similarities of full-sentence definitions and spoken folk definitions go beyond the use of informal, everyday language and the focus on typical senses. Folk definitions also embed information about the context in which a word is used. Casagrande and Hale (1967) found that definitions for particular semantic categories used similar grammatical structures: for example, the contingency type of definition (which states a necessary condition) was usually introduced with a relative clause similar to an 'if' or 'when' clause in English. Sinclair (1987 b) and Hanks (1987) argued that such grammatical patterns were inseparable from the meaning of the words. Defining a word in a full sentence allows lexicographers to show typical grammatical patterns and collocations in a way that is easily understood by learners. For example, Hanks (1987) showed that a very simple distinction between the introduction of the definitions for 'brick' and 'calligraphy' differentiates effectively between countable nouns ("A brick is....") and uncountable nouns ("Calligraphy is ...."). Barnbrook (2002) further described the systematic use of particular constructions in the full-sentence definitions of different word categories. Such definitions are especially informative for encoding.

Although he agreed with the theoretical principles that led to the full-sentence definition format, Rundell (2006), argued that this format should not be universally applied to all words. Rundell noted that full sentence definitions tended to be longer and therefore placed a larger processing burden on language learners, even though the wording of the definition 
itself might be simple. While mostly the additional length is justified by the detailed information provided, in cases where the co-text was not significant, nothing was gained by insisting on a full-sentence definition. As was mentioned before, Stock (1988) found that folk definitions were in fact sometimes very short and did not always repeat the word being defined. This suggests that the full-sentence definition format, when applied universally, loses some of the features of folk definitions that are potentially useful to learners, especially in decoding and vocabulary learning.

Although these criticisms warrant caution against the universal use of full-sentence definitions, Rundell acknowledged the huge positive influence of this format on recent learners' dictionaries. The inclusion of this folk definition-inspired format alongside other techniques has spearheaded a general call for traditional definitions to be replaced by easyto-understand 'explanations' providing detailed information for encoding and decoding, and pointing to the most typical and frequent usage patterns (e.g. Atkins \& Rundell, 20o8; Hanks, 2015). Furthermore, Stock (1992) argued that the inclusion in such explanations of cultural connotations is crucial for decoding learners, even when they already understand the denotational meaning of the word. Atkins and Rundell (2008) similarly suggested that cultural connotations and sensitivities, as well as the pragmatic force of certain words, should be integrated in definitions, although they noted that it may be difficult to determine which cultural connotations are essential for learners to know.

These developments show that there are precedents for using folk definitions as a model for dictionary definitions. Their application in sign language dictionaries, however, will differ in at least three major respects. Firstly, as was described in section 3.4.9 - Corpora and corpusbased lexicography, sign language dictionaries cannot draw on large corpora to determine typical usage. Elicited signed folk definitions will therefore not only function as a template for the format of the eventual dictionary definitions, but they will also be the main source of data for how the sign is used in context. Secondly, unlike the edited written full sentence definitions in English learner's dictionaries, signed dictionary definitions will need to be reperformed by an individual in order to be fixed on video. While some editing is possible, the end product will therefore retain some features of spontaneous production. Finally, there are still many uncertainties about the status of certain lexical items in sign languages. As was described in section 2.3 - Summary and discussion, it is often not possible to allocate signs to a particular word class or even to state with certainty that they are completely specified. These factors make it improbable that a completely consistent system of different sentence formats 
could be devised to show nuanced grammatical information. Nevertheless, some common sentence patterns or co-text preferences may emerge from an analysis of folk definitions given by multiple sign language users.

Returning briefly to the user needs that signed dictionary definitions are ultimately intended to meet, it is worth noting that video clips showing a sign model, being closer in form to spontaneous spoken explanations than to the structured written full sentence definitions, potentially serve as better tools for vocabulary learning. McKeown (1993), for example, noted that dictionary definitions in themselves would not be sufficient stimuli for vocabulary learning. She suggested, however, that dictionary definitions could be used by teachers in conjunction with spoken explanations:

"Teachers might promote their students' learning by transforming definitions into explanations that characterize a word's prototypical use in readily comprehensible language. There could be much value for a language learner in a restatement of a definition by an experienced language user."

As was discussed in section 4.6.4 - Problems and issues, language learners using the Online Dictionary of NZSL sometimes treated the video images as if they were a person in a live interaction, addressing comments to the video as well as copying the signs in the example. This suggests the possibility that watching signed explanations on video has similar benefits to those listed for spoken explanations in section 6.2 - Spoken definitions and explanations.

\subsection{Signed definitions and explanations}

Although to my knowledge there are no dedicated studies of folk definitions in sign languages, there are references to signed explanations by Deaf adults in the wider sign language studies literature. Casagrande and Hale (1967) referred to explanations of word meaning as a "universal linguistic need" (p. 165), and it is indeed clear that the contexts in which Deaf people are called upon to explain the meaning of signs are similar to those contexts in which spoken folk definitions were found to occur. These contexts span from informal everyday conversation to formal educational settings. The need to explain word or sign meaning typically arises in interactions with language learners, whether children learning a first language from their parents, children acquiring literacy at school, students in classes taught in a foreign language, or adult researchers involved in learning and documenting a community's language and culture. However, there are some sociolinguistic circumstances that may affect the way in which such explanations are given by Deaf people in 
everyday contexts and that therefore have implications for eliciting signed folk definitions in the current study.

First of all, folk definitions are usually explanations by native speakers of a language. The sociolinguistic context of sign languages that I described in section 2.1.1 - Origins of natural sign languages means that there are few truly native sign language users: the majority of adults who use a sign language as their main or preferred language will not have learned the language until a later age. Richards and Taylor (1992) found that linguistic proficiency of the speaker was one of the factors that influenced the strategies chosen to explain word meaning. It is therefore possible that there will be more individual variation in the strategies elicited in the current study than was found in studies of spoken folk definitions.

Secondly, the continuous contact between Deaf sign language users and the surrounding spoken language means that many Deaf adults are to some extent bilingual. In contexts where explanations of the meaning of signs are requested by a hearing person (for example, a student in a sign language class or a hearing researcher), Deaf people may therefore use speech or writing instead. While spoken folk definition strategies also included providing an equivalent in the target audience's first language (e.g. Chaudron, 1982; Nation, 2001), for many Deaf people such strategies might be a more natural response than signing their explanations. Even if explanations are signed, they are likely to show more features of the non-native lexicon such as loan translations, fingerspelling or mouthing (see section 2.2.5 The non-native lexicon). For the purpose of the current study, even though the envisaged end users of signed folk-based dictionary definitions are hearing language learners, definitions should be modelled on explanations given to other Deaf sign language users.

Studies of Deaf adults interacting with deaf children or young students have primarily focused on overall 'scaffolding' strategies including features of child directed signing (e.g. Neese Bailes, Erting, Erting, \& Thumann-Prezioso, 2009) and explanations of written or spoken words through the medium of the sign language, rather than on explanations of sign meaning per se. These strategies, however, have many characteristics in common with folk definitions: for example, Smith (2003) showed that Deaf teachers provided short, clear explanations aimed at solving immediate comprehension problems in the context of another task by giving signed equivalents for written words (e.g. the NZSL sign BOx for the English word 'carton') and by relating words to children's real-world experiences. Longer elaborations included showing real-life objects as well as using a strategy referred to as 
'chaining' (Humphries \& MacDougall, 1999) to link together different representations of the same concept, for example by showing a written word, fingerspelling it, mouthing the word, and providing a signed equivalent.

Situations where Deaf sign language users learn another sign language are becoming more common, mostly as part of increased migration and travel as well as exposure to foreign sign languages through video sharing via the internet. Hessman and Pyfers (2014) reported on a unique formal online course, Signs 2 Go, aimed at Deaf learners of British Sign Language (BSL). Lessons in the course were based on video clips showing interviews and dialogues between BSL users, with a BSL teacher providing explanations of vocabulary used in the dialogues. Hessman and Pyfers compared the explanations provided by the BSL teacher with the parallel explanations in one of the target groups' first language, German Sign Language (DGS). Their analysis is the most complete study of meaning explanation strategies in sign languages to date, and it is significant that many of the strategies reported correspond closely with Chaudron's (1982) account of strategies used by English teachers. For example, Hessman and Pyfers noted that synonyms, paraphrases and extended examples were common strategies used in both the BSL and DGS explanations, and that the DGS explanations added English equivalents (through fingerspelling) as well as giving DGS equivalents. These parallels suggest that the benefits of spoken explanations as a model for foreign language users and for dictionaries that were noted in the previous sections will also be applicable to signed explanations in sign language dictionaries.

Hessman and Pyfers also found evidence of strategies specific to the signed modality, such as making explicit iconic and motivated features of the sign (see section 2.2.3.3 - Iconicity). The teachers frequently commented on language variation and on features of the dialogues that showed language contact between BSL and English, such as fingerspelling and mouthing. In their explanations of the meaning of some signs they also commented on the grammatical usage of these signs, for example demonstrating inflections, plural forms, and non-manual features that were considered an integrated part of the sign or provided adverbial or intensifying information. Finally, the explanations included contextual information such as English abbreviations and geographical locations, and cultural information such as the role of Deaf clubs and BSL users' strategies to get someone's attention before starting to sign.

One key difference to note is that Deaf people have been found to communicate much more easily with users of other sign languages than two speakers of mutually unintelligible spoken 
languages would be able to do. Supalla and Webb (1995) found that there was considerable overlap in the grammatical devices used in different sign languages, and that this enabled Deaf users of different sign languages to use complex grammatical structures such as inflected verb morphology and various types of negation in ad hoc communication with each other. Hessman and Pyfers (2014) similarly found that the Deaf teachers in the online course assumed that many aspects of grammar would be understood by foreign Deaf learners of BSL. The implications for the current study are that native Deaf sign language users' signed explanations are likely to include grammatical constructions that are potentially valuable as models for hearing learners' language production, but that at the same time might be too complex for these learners to understand. For this reason, McKee and McKee (2013) commented on the avoidance of some of these structures in signed example sentences aimed at hearing learners. The final form of signed dictionary definitions will need to balance the main end-user group's comprehension and production needs.

As well as demonstrating that signed explanations share common qualities with spoken folk definitions, the study by Hessman and Pyfers also shows that these qualities of 'live', informal explanations were preserved when the BSL teacher was recorded on video. The video explanations in Signs 2 Go are therefore a useful starting model for folk-derived definitions in a sign language dictionary. However, since in Signs 2 Go these explanations are provided for signs used in a video dialogue, they will be different from the context-free presentation of signs in a dictionary.

Since signed dictionary definitions to date have been very rare, there are few accounts of the processes used to create these definitions and their final form. Brien and Collins (1997) reported on discussions held amongst a group of eight Deaf and eight hearing researchers during an international workshop that led to decisions on an appropriate format for definitions in the BSL CD-ROM Dictionary of Deaf Community and Culture (Brien et al., 1997). Brien and Collins' finding that definitions generated by Deaf sign language users themselves revealed a unique conceptual base supports one of the key arguments for using such native user paraphrases as the basis for dictionary definitions. Other findings reported by Brien and Collins included a greater focus to the role of context in the signed definitions. This finding may be a result of the previously mentioned link between definition style and formal education and literacy, with Kurland and Snow (1997) reporting longer, more narrative-like, context-embedded explanations by speakers who had not been exposed to formal style definitions. Bienvenu (2003) remarked that a number of the Deaf people in an 
evaluation group for a pilot monolingual ASL dictionary did not immediately grasp the purpose of definitions, partly as a result of their limited experiences as dictionary users. Attempting to model signed definitions on a formal definition format is therefore not only less reflective of natural and typical language use by Deaf people, but also runs counter to recent trends in general lexicography that have favoured informal explanations with a larger focus on typical word contexts (see the previous section). Brien and Collins noted that a definition style emerged from their dictionary project that was evaluated as appropriate and comprehensible by other Deaf people. The aim of the current study is to identify in more detail the features of such a definition style and to suggest a methodology for creating dictionary definitions based on these features.

\subsection{Research questions}

The study in this chapter was intended to answer the following research questions:

- Can common semantic, grammatical or discourse features be identified in signed folk definitions?

- Which features of (spontaneous) signed folk definitions could be carried over in (planned) signed dictionary definitions?

\subsection{Methodology of folk definition studies}

An important difference between the current study and previous projects involving signed definitions is that I propose to separate the collection and analysis of lay folk definitions from the creation of dictionary-ready signed definitions. By first collecting folk definitions from a cross-section of Deaf sign language users, common patterns of use can be identified in a more objective way than definitions created by a single Deaf person allow. In addition, the collected folk definitions can be a useful source of data in themselves, beyond their use in a dictionary or learning resource.

The studies of folk definitions cited in the literature review in this chapter used two distinct methods. Some of these studies (e.g. Chaudron, 1982; Flowerdew, 1992) recorded folk definitions they observed occurring in spontaneous discourse. Other studies (e.g. Casagrande \& Hale, 1967; Manes, 1980; Dingemanse, 2015) elicited definitions by presenting native speakers with a pre-determined list of words. Given the context-embedded features of folk definitions, the observation method would at first sight be preferable; however, when the 
purpose of the study is to concentrate on specific vocabulary (such as Dingemanse's study on ideophones), this method cannot be relied on. Manes (1980) compared spontaneous definitions with definitions elicited from a word list and found that they were very similar in structure and content. For the current study, elicitation of definitions through presenting a list of signs via video clips was therefore the most suitable method.

As was discussed in the previous section, folk definitions addressed to a hearing person might show influence of language contact and non-sign language communication to a greater extent than desirable. The elicitation of signed folk definitions in this study therefore would benefit from the presence of other Deaf people as conversation partners. A small pilot elicitation session with a group of 10 Deaf teachers showed that an informal discussion format with other Deaf people worked well, especially if one of the participants wasn't familiar with a particular sign, since this required genuine explanations in an authentic situation. However, some participants' contributions dominated the discussion while other participants would take a back seat and when prompted, tended to agree with previous contributions rather than offering their own explanations. I therefore decided to hold individual elicitation interviews, with a Deaf person acting as the interviewer and main conversation partner.

\subsection{Method}

\subsubsection{Materials}

Table 6.1 shows the 15 target signs that were chosen as the stimulus for eliciting folk definitions. These signs were selected from the lower-scoring test items that were found to be in need of further dictionary information in the test reported on in the previous chapter (Chapter 5 - Evaluating the user need for definitions). In order to ensure that the test could be re-administered to evaluate the effect of signed definitions, it was necessary to select target signs evenly from test blocks A-E, from both the comprehension and production halves of the test, and to ensure that the six different categories (culture-bound, idiomatic, polysemous, metaphoric/metonymic, vocabulary type / word class, and other) were represented. The selected target signs were therefore not the absolute lowest-scoring items in the test. However, all selected items scored below 0.78 indicating that at least 2 students had not identified the error for that item in the test. Where the test item involved multiple dictionary entries (for example, where the sign had one or more homonyms or where the test item involved comparing two sign variants with different grammatical or collocational behaviour), only one sign video was presented as a stimulus for definition. 


\begin{tabular}{|c|c|c|}
\hline Test item no. & Sign (drawing) & Sign (video) \\
\hline AC4 KEEP-INSIDE-ONESELF & & $\begin{array}{l}\text { http://freelex.nzsl.vuw.ac.nz/dnzsl/freelex/assets/1157/ } \\
\text { keep_inside_oneself.1157.main_glosses.rp.r480x360.mp4 }\end{array}$ \\
\hline $\begin{array}{l}\text { AC5 PLAIN / CONFIDENTIAL, } \\
\text { PRIVATE }\end{array}$ & & $\begin{array}{l}\text { http://freelex.nzsl.vuw.ac.nz/dnzsl/freelex/assets/266/ } \\
\text { plain.266.main_glosses.rp.r480x360.mp4 }\end{array}$ \\
\hline AP1 PUT-ASIDE & & $\begin{array}{l}\text { http://freelex.nzsl.vuw.ac.nz/dnzsl/freelex/assets/512/ } \\
\text { apart_from.512.main_glosses.sp.r480x360.mp4 }\end{array}$ \\
\hline BC2 UNINTELLIGIBLE-VOICE & & $\begin{array}{l}\text { http://freelex.nzsl.vuw.ac.nz/dnzsl/freelex/assets/5167/ } \\
\text { deaf_voice.5167.main_glosses.sp.r480x360.mp4 }\end{array}$ \\
\hline $\begin{array}{l}\text { BC5 SMALL-ROUND-FOOD- } \\
\text { PLURAL }\end{array}$ & & $\begin{array}{l}\text { http://freelex.nzsl.vuw.ac.nz/dnzsl/freelex/assets/3137/ } \\
\text { radish.3137.main_glosses.rp.r480x360.mp4 }\end{array}$ \\
\hline BP5 DEAF & & $\begin{array}{l}\text { http://freelex.nzsl.vuw.ac.nz/dnzsl/freelex/assets/887/ } \\
\text { full_deaf.887.main_glosses.vs.r480x360.mp4 }\end{array}$ \\
\hline CC1 ASK / REMIND / TELL & & $\begin{array}{l}\text { http://freelex.nzsl.vuw.ac.nz/dnzsl/freelex/assets/1071/ } \\
\text { ask.1071.main_glosses.mb.r480x360.mp4 }\end{array}$ \\
\hline CC5 DEVELOP / GROW & & $\begin{array}{l}\text { http://freelex.nzsl.vuw.ac.nz/dnzsl/freelex/assets/3567/ } \\
\text { grow.3567.main_glosses.mb.r480x360.mp4 }\end{array}$ \\
\hline CP5 HEARING & & $\begin{array}{l}\text { http://freelex.nzsl.vuw.ac.nz/dnzsl/freelex/assets/2117/ } \\
\text { hearing.2117.main_glosses.jo.r480x360.mp4 }\end{array}$ \\
\hline DC1 TALK-IN-SIGN-LANGUAGE & & $\begin{array}{l}\text { http://freelex.nzsl.vuw.ac.nz/dnzsl/freelex/assets/1300/ } \\
\text { converse.1300.main_glosses.sp.r480x360.mp4 }\end{array}$ \\
\hline $\begin{array}{l}\text { DC3 BEEN-TO / } \\
\text { HAVE-EXPERIENCE-OF }\end{array}$ & & $\begin{array}{l}\text { http://freelex.nzsl.vuw.ac.nz/dnzsl/freelex/assets/4794/ } \\
\text { been_to.4794.main_glosses.mb.r480x360.mp4 }\end{array}$ \\
\hline DP5 DECREASE & & $\begin{array}{l}\text { http://freelex.nzsl.vuw.ac.nz/dnzsl/freelex/assets/249/ } \\
\text { decrease.249.main_glosses.rp.r480x360.mp4 }\end{array}$ \\
\hline $\begin{array}{l}\text { EC1 ONE-HANDED- } \\
\text { FINGERSPELLING }\end{array}$ & & $\begin{array}{l}\text { http://freelex.nzsl.vuw.ac.nz/dnzsl/freelex/assets/1814/ } \\
\text { ASL fingerspelling.1814.main glosses.mb.r480x360.mp4 }\end{array}$ \\
\hline $\begin{array}{l}\text { EC4 I-WAS-WRONG / YOU- } \\
\text { GOT-ME }\end{array}$ & & $\begin{array}{l}\text { http://freelex.nzsl.vuw.ac.nz/dnzsl/freelex/assets/6089/ } \\
\text { i_was_wrong.6089.main_glosses.spa.r480x360.mp4 }\end{array}$ \\
\hline EP2 SPEECH & & $\begin{array}{l}\text { http://freelex.nzsl.vuw.ac.nz/dnzsl/freelex/assets/2207/ } \\
\text { speak.2207.main_glosses.rp.r480x360.mp4 }\end{array}$ \\
\hline
\end{tabular}

Table 6.1: 15 stimulus items used to elicit folk definitions 


\subsubsection{Participants}

A total of 13 participants were recruited through personal networks. Selection of participants took into account the sociolinguistic factors discussed in section 6.4 - Signed definitions and explanations. Although efforts were made to select a cross-section of participants from different regions, ages and genders, the overriding criteria were native-like use of NZSL (with priority given to Deaf people from Deaf families) and some familiarity with explaining the meaning of signs. All participants used NZSL as their main or preferred language on a daily basis and had been born in New Zealand or lived here since childhood. The majority of participants were NZSL teachers involved in teaching hearing L2 learners. Other participants had experience of explaining sign meaning through their work supporting deaf school students, giving formal presentations or producing instructional videos.

While participants were only recruited in two regions, Auckland and Wellington, several participants had previously lived or been educated in other parts of New Zealand, including in the South Island.

Table 6.2 summarises the demographics of the participant groups in the two regions.

\begin{tabular}{lll}
\hline & Wellington $(\mathbf{n}=6)$ & Auckland $(\mathbf{n}=\mathbf{7})$ \\
\hline Age & 3 & 1 \\
\hline Younger (<30 years) & 2 & 2 \\
Mid (30-50 years) & 1 & 4 \\
Older (>50 years) & 3 & \\
\hline Gender & 3 & 0 \\
Male & 3 & 7 \\
Female &
\end{tabular}

Table 6.2: Demographics of participants in the folk definition elicitation study

\subsubsection{Procedure}

Elicitation interviews took place in various locations in Wellington and Auckland at participants' places of work or meeting rooms. In Wellington, the interviews were conducted by a Deaf NZSL user while I was present to film and to explain the purpose of the study. In Auckland, I was unable to arrange for a Deaf interviewer. In two cases I therefore interviewed participants together (as a pair and a group of three). The three remaining individuals were 
interviewed by myself. In the data analysis I took into account that my status as a hearing NZSL user may have influenced the register used in these three interviews.

Interviews were recorded on video. Although all participants were used to being recorded in the course of their work, the presence of video cameras may inhibit informal language production at first. A 5-10 minute warmup conversation was therefore held with participants before the elicitation started. The warmup conversation was deleted from the data before analysis.

After the warmup conversation, participants were presented with each of the 15 signs in turn and asked to explain the meaning of that sign. Presentation of the stimulus was in the form of the video for that sign entry in the ODNZSL (see table 6.1). In a few cases where participants did not spontaneously provide an explanation for a sign, the Deaf interviewer used prompts such as 'Do you use this sign yourself?; 'Who uses this sign?'; and 'Are there other signs with this sense?'

The elicitation interviews were approximately one hour in length.

\subsubsection{Analysis}

The elicited folk definitions were coded using NVIVO qualitative data analysis software version 11 for Mac (QSR International, 2015). Semantic categories were assigned on the basis of Casagrande and Hale's (1967) categorisation of semantic relationships found in Papago folk definitions, since their landmark study has been the foundation of most research on folk definitions. Common grammatical patterns and occurrences of fingerspelling and mouth patterns were also coded. Any signs that were used by more than one person in their explanation of the meaning of the target sign, either as synonyms or as collocations, were noted, as well as any comments on sociolinguistic and situational features such as register, regional and age variation.

Although Casagrande and Hale (1967) created an exhaustive list of 13 categories to account for all semantic relationships found in their data, they acknowledged that further semantic relationship types might be identified in other languages, and suggested that data on folk definitions in other languages was needed to answer questions about the cognitive nature of such definitions, for example whether universal semantic types could be identified, or whether preferences for certain semantic types might reflect differing world views. Later studies of folk definitions have shown that there are indeed remarkable similarities in 
semantic types used in folk definitions in different languages (Manes, 1980). Newport and Bellugi (1978) demonstrated that prototypical semantic relationships, including superordinate and subordinate levels, were also expressed in American Sign Language.

It has been shown that particular semantic categories may be associated with defining particular word classes (Casagrande \& Hale, 1967; Mathiot, 1979; Manes, 1980). As was discussed in the literature review, there is also considerable overlap between folk definitions provided by 'linguistically naive' speakers of unwritten languages and informal explanations by teachers in academic discourse (e.g. Chaudron, 1982; Richards \& Taylor, 1992; Flowerdew, 1992). Although these later authors have developed their own categorisations, expanding on or conflating some of Casagrande and Hale's semantic relationship types, the correspondences between different categorisations are large enough to take the original 13 categories as a starting point in exploring the semantic content of signed folk definitions. Table 6. 3 shows the types of semantic relationships in folk definitions as outlined by Casagrande and Hale. 


\begin{tabular}{|c|c|c|}
\hline $\begin{array}{l}\text { Semantic } \\
\text { relationship } \\
\text { type }\end{array}$ & $\begin{array}{l}\text { Relationship between } X \text { (the word } \\
\text { being defined) and } Y \text { (the terms } \\
\text { used to define it) }\end{array}$ & $\begin{array}{l}\text { Common features, or contexts in which this } \\
\text { relationship occurs }\end{array}$ \\
\hline Attributive & $\begin{array}{l}X \text { is defined with respect to one or } \\
\text { more distinctive or characteristic } \\
\text { attributes } Y\end{array}$ & $\begin{array}{l}\text { Commonly used to define nouns. } \\
\text { Attribute types: } \\
\text { - intrinsic or extrinsic stimulus properties } \\
\text { - distinctive markers } \\
\text { - habitat } \\
\text { - behaviour or action } \\
\text { - age, sex, line of descent, generation }\end{array}$ \\
\hline Contingency & $\begin{array}{l}\mathrm{X} \text { is defined with relation to a usual } \\
\text { or necessary antecedent or } \\
\text { concomitant } \mathrm{Y}\end{array}$ & $\begin{array}{l}\text { - X usually or necessarily follows an event } \mathrm{Y} \text {, or } \\
\text { happens at the same time as } Y \\
\text { - May be introduced by a conditional clause }\end{array}$ \\
\hline Function & $\begin{array}{l}X \text { is defined as the means of } \\
\text { effecting } Y\end{array}$ & $\begin{array}{l}\text { - we perform } Y \text { with (the aid of) } X \\
\text { - } Y \text { is performed with the aid of } X \\
\text { In Papago, usually a nominalisation of one of } \\
\text { these sentence structures }\end{array}$ \\
\hline Spatial & $\begin{array}{l}\mathrm{X} \text { is oriented spatially with respect } \\
\text { to } \mathrm{Y}\end{array}$ & $\begin{array}{l}\text { - Uses locatives (under, on, above) } \\
\text { - X's 'stance' often included as an attribute, e.g. } \\
\text { X stands, protrudes, sits, lies }\end{array}$ \\
\hline Operational & $\begin{array}{l}X \text { is defined with respect to an } \\
\text { action } Y \text { of which it is a } \\
\text { characteristic goal or recipient }\end{array}$ & \\
\hline Comparison & $\begin{array}{l}\mathrm{X} \text { is defined in terms of its } \\
\text { similarity and/or contrast with } \mathrm{Y}\end{array}$ & $\begin{array}{l}\text { - } \mathrm{X} \text { is like } \mathrm{Y} \\
\text { - } \mathrm{X} \text { looks like } \mathrm{Y} \\
\text { - } \mathrm{X} \text { and } \mathrm{Y} \text { are often in the same position in a folk } \\
\text { hierarchy }\end{array}$ \\
\hline Exemplification & $\begin{array}{l}\mathrm{X} \text { is defined by citing an } \\
\text { appropriate co-occurrent, } \mathrm{Y}\end{array}$ & $\begin{array}{l}\text { Reverse of attributive definition, used for nouns or } \\
\text { adjectives: } \\
\text { - } X \text { is itself an attribute of } Y \text {; or } \\
\text { - } Y \text { characteristically possesses } X\end{array}$ \\
\hline Class inclusion & $\begin{array}{l}X \text { is defined with respect to its } \\
\text { membership in a hierarchical class } \\
Y\end{array}$ & $\begin{array}{l}\text { - } \mathrm{X} \text { is a } \mathrm{Y} \text { (cf. 'naming'); } \\
\text { - may co-occur with attributive definition: } \mathrm{X} \text { is a } \\
\mathrm{Y} \text { which ... (cf. formal definition) }\end{array}$ \\
\hline Synonymy & $\begin{array}{l}X \text { is defined as being equivalent to } \\
Y\end{array}$ & $\begin{array}{l}\text { - } X \text { and } Y \text { may not always be substitutable; } \\
\text { - } X \text { and } Y \text { may be equivalents from different } \\
\text { dialects }\end{array}$ \\
\hline Antonymy & $\begin{array}{l}\mathrm{X} \text { is defined as the negation of } \mathrm{Y} \text {, its } \\
\text { opposite }\end{array}$ & $\begin{array}{l}\text { - Often (almost always in Papago) used to define } \\
\text { adjectives }\end{array}$ \\
\hline Provenience & $\begin{array}{l}X \text { is defined with respect to its } \\
\text { source } Y\end{array}$ & - $X$ comes from / out of $Y$ \\
\hline Grading & $\begin{array}{l}X \text { is defined with respect to its } \\
\text { placement in a series or spectrum } \\
\text { that also includes } Y\end{array}$ & $\begin{array}{l}\text { - used e.g. in listing days of the week, or colours } \\
\text { in a spectrum }\end{array}$ \\
\hline Circularity & $X$ is defined as $X$ & $\begin{array}{l}\text { - adds no further meaning; not strictly a } \\
\text { definition or explanation }\end{array}$ \\
\hline
\end{tabular}

Table 6.3: Semantic relationship types of folk definitions (Adapted from Casagrande and

Hale, 1967) 


\subsection{Results and discussion}

As was mentioned in section 3.2.1 - Representing signs without a written form, transcribing signs through English glosses is less than ideal since this method runs the risk of conflating the semantics and grammatical properties of the sign with those of the English word chosen to represent the sign. However, for ease of reference and to preserve the anonymity of the participants, data in this section will mainly be presented in transcribed form. Video clips of key examples will be available to examiners on a separate disk.

Transcription conventions can be found in the front matter of this thesis (Transcription conventions).

As was found in Casagrande and Hale's (1967) data and also remarked on by Kurland and Snow (1997), many folk definitions are narrative-like and compound in nature. In other words, it is common for people to use several strategies and to focus on different aspects of a word or sign's meaning in turn. Examples in this section will only describe the part of the folk definition that is relevant to the particular semantic category under discussion.

\subsubsection{Semantic categories}

Examples of all semantic relationships from Casagrande and Hale's categorisation were found in the data. However, the frequency with which particular categories occurred differed from Casagrande and Hale's findings (table 6.3 listed the semantic categories roughly in order of frequency, i.e. the 'attributive' relationship occurred the most often in the Papago data).

Figure 6.1 shows which semantic categories were used in the folk definitions for the 15 target signs. The most frequently used categories were synonymy and exemplification, followed by the comparison and attributive categories. Antonymy and spatial categories were used for three target signs each. The remaining categories were each used for only one target sign. 


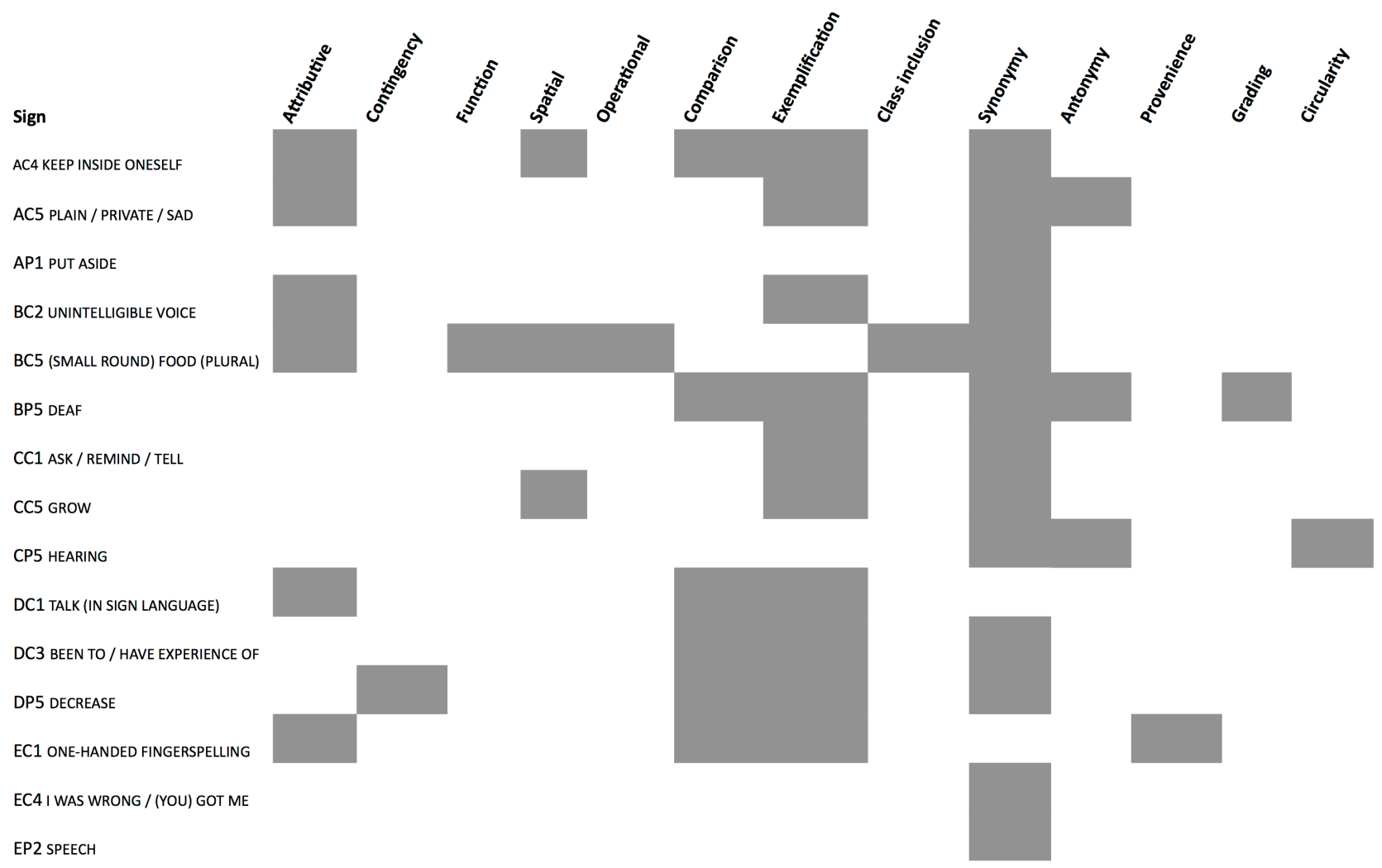

Figure 6.1: Semantic categories used in NZSL folk definitions for 15 target signs

\subsubsection{Synonymy}

In section 5.2.1 - Degrees of equivalence, it was stated that equivalence between two words in separate languages is rare. The same holds true for synonymy, or equivalence between two words in the same language. Nevertheless, providing a synonym or 'equivalent' was found to be a common strategy of spoken explanations (e.g. Chaudron, 1982). Casagrande and Hale (1967) acknowledged that synonyms in folk definitions were not exactly the same in meaning, nor were the lexemes offered as synonyms necessarily grammatically substitutable for the item being defined. The semantic category of 'synonymy' therefore does not imply equivalence, but only that the person providing a folk definition recognises a strong similarity in meaning or use between the 'synonyms'.

Figure 6.2 shows the signs that were provided as synonyms or near-synonyms in response to 13 of the target signs. 
AC4 KEEP-INSIDE-ONESELF

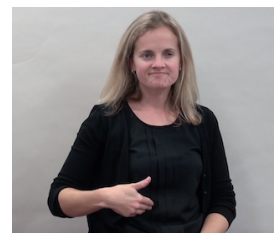

GUT-FEELING

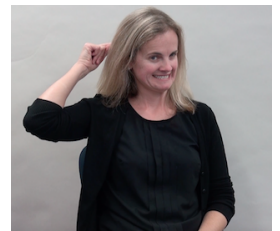

NIGGLING-THOUGHT

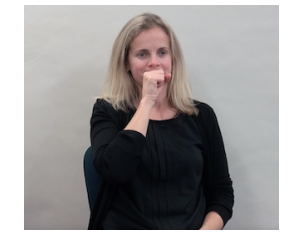

BITE-ONE'S-TONGUE

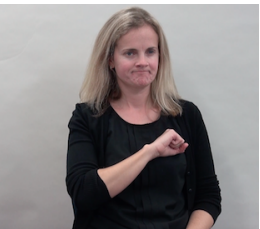

FEEL-HURT

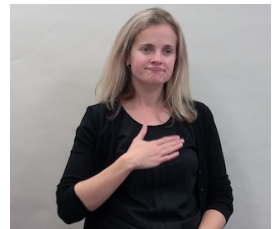

PUT-UP-WITH

\section{AC5 PLAIN / CONFIDENTIAL, PRIVATE}

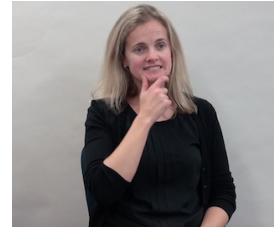

SAD

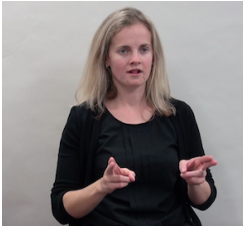

NORMAL

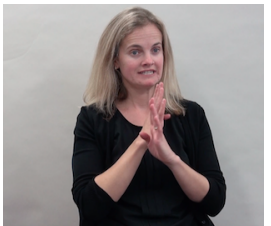

PRIVATE

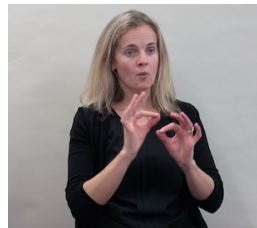

QUIET

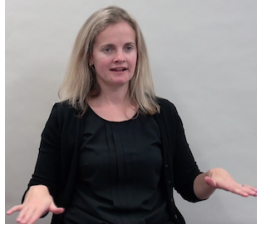

PLAIN

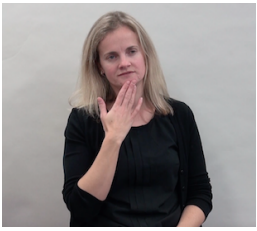

BORING

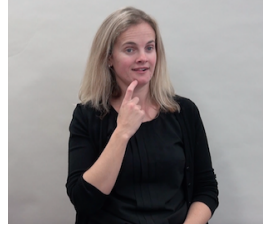

SIMPLE

AP1 PUT-ASIDE

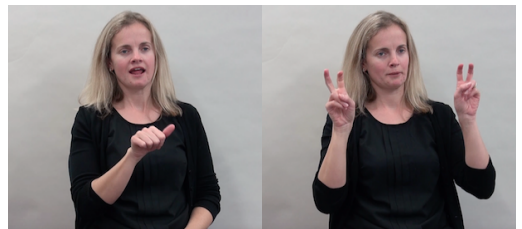

NEXT TOPIC

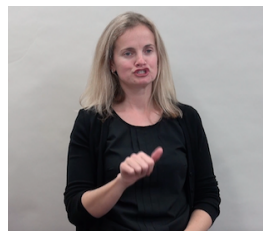

NEXT-TURN

BC2 UNINTELLIGIBLE-VOICE

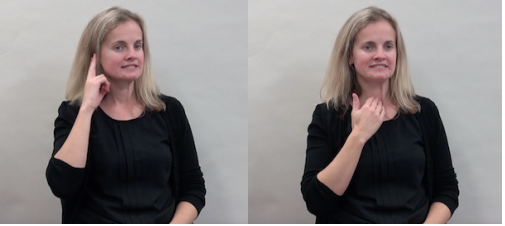

DEAF-VOICE

\section{BC5 SMALL-ROUND-FOOD-PLURAL}

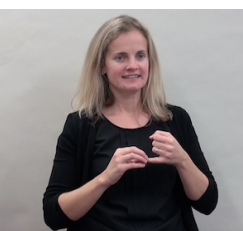

BERRY /

STRAWBERRY

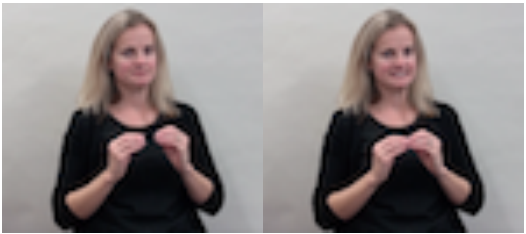

BAKED BEANS 1

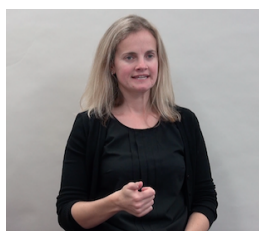

PEA

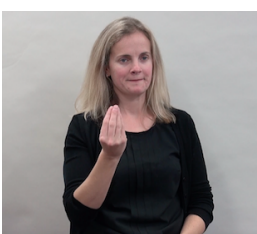

GRAPES 1

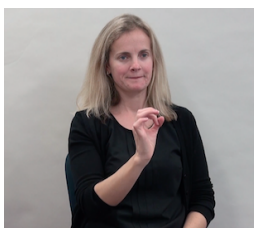

GRAPES 2

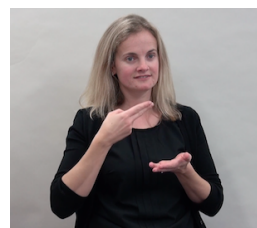

RICE

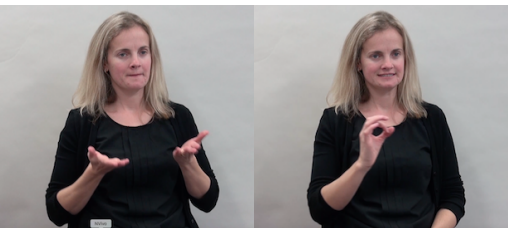

BAKED BEANS 2

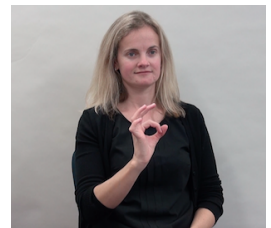

SMALL-ROUNDFOOD-SINGULAR 


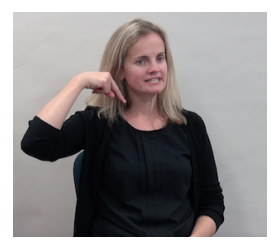

CHERRY 1

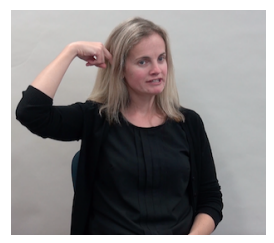

CHERRY 2

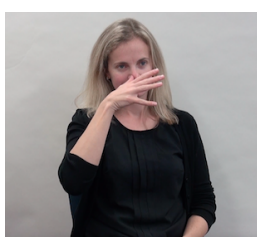

OYSTER

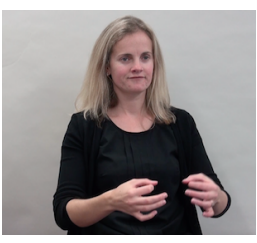

OLIVES 1

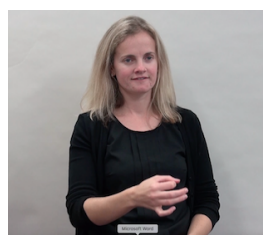

OLIVES 2

\section{BP5 DEAF}

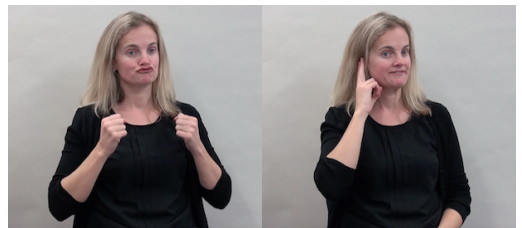

STRONGLY / VERY DEAF

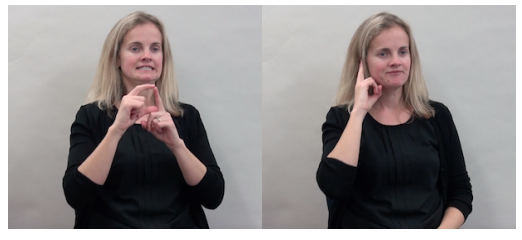

'CAPITAL-D' DEAF

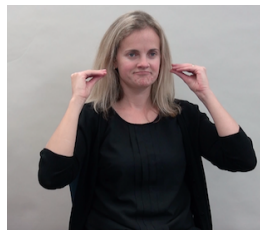

COMPLETEHEARING-LOSS

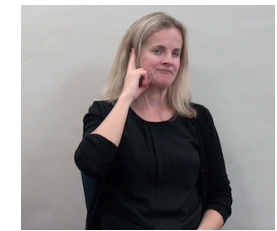

DEAF-with-emphasis

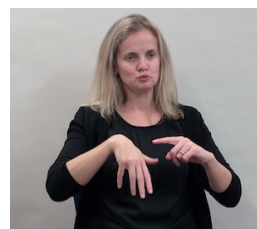

GRASSROOTS

CC1 ASK / REMIND / TELL

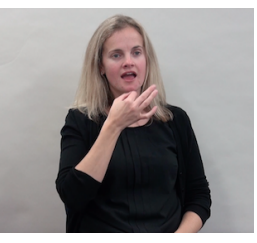

ASK 2

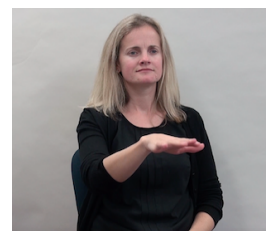

HEY

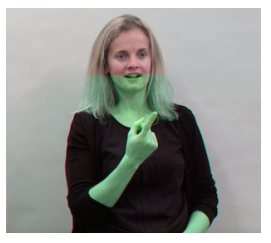

TELL

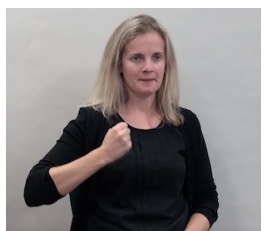

STAMP

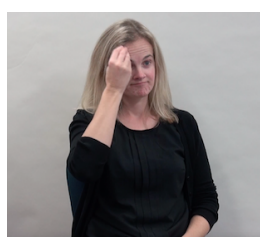

INFORM

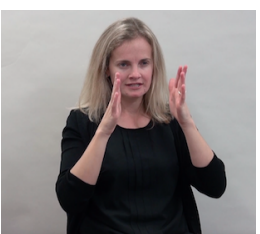

WANT ATTENTION

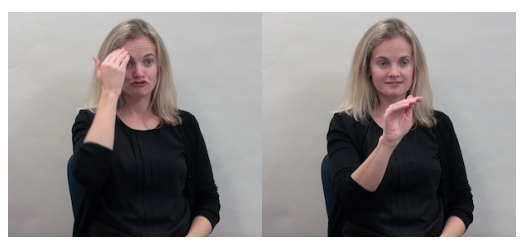

REMIND

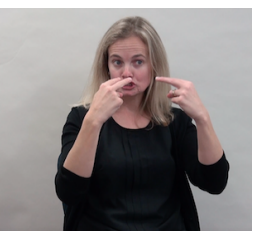

WATCH-ME

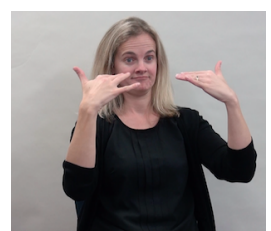

ATTRACTATTENTION

\section{CC5 DEVELOP / GROW}

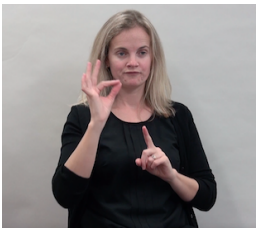

IMPROVE

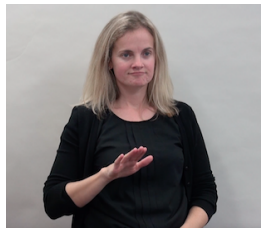

GO-UP

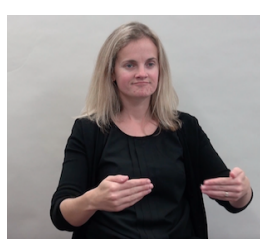

GO-AHEAD

\section{CP5 HEARING}

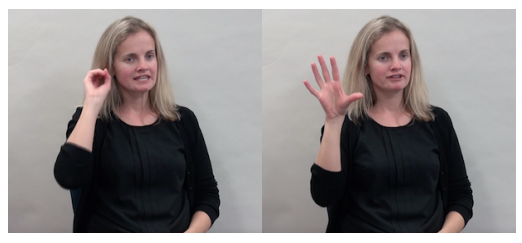

HEARING 1

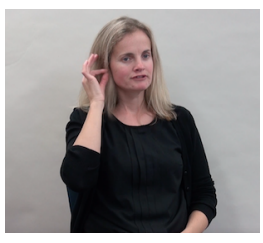

HEARING 2

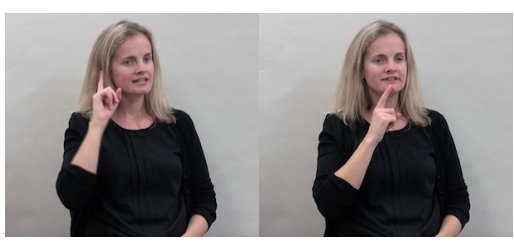

HEARING 3 


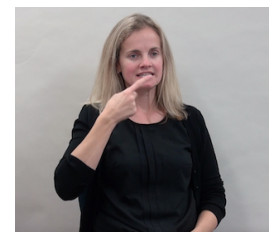

HEARING ASL

DC3 BEEN-TO /HAVE-EXPERIENCE-OF

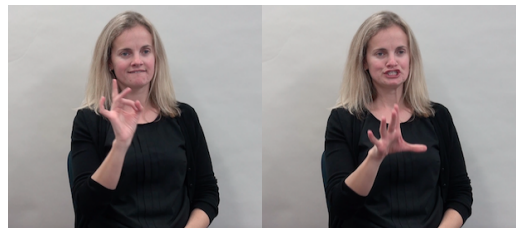

HAVE-DONE-THAT

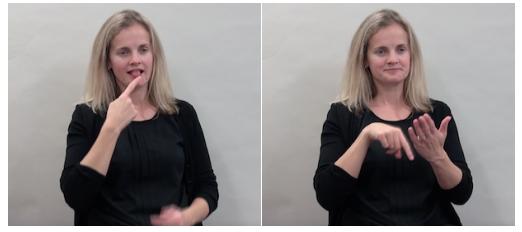

CROSS-SOMETHING-OFF-A-LIST

\section{DP5 DECREASE}

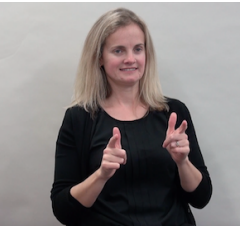

LESS

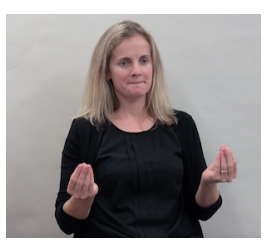

DISAPPEAR

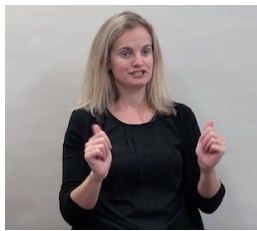

FINISH / THEN

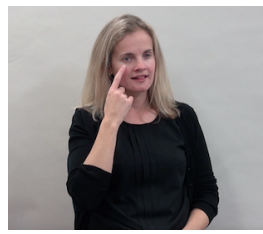

HAVE-A-LOOK / TRY

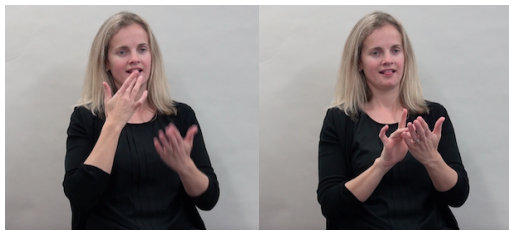

BEEN-TO / HAVE-EXPERIENCE-OF 2

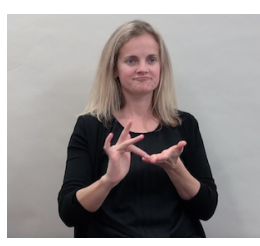

EMPTY

\section{EC4 I-WAS-WRONG / YOU-GOT-ME}

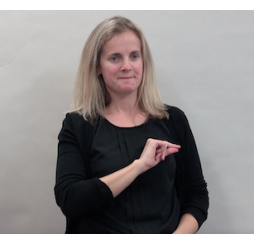

FEEL-SMALL

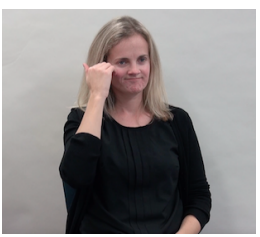

EMBARRASSED

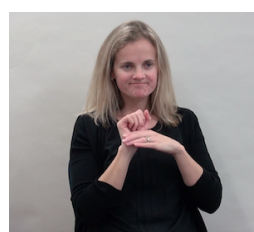

HUMILIATED

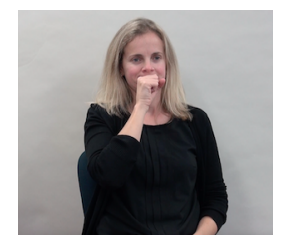

BITE-ONE'S-TONGUE

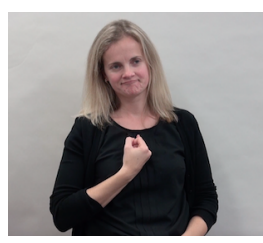

OOPS

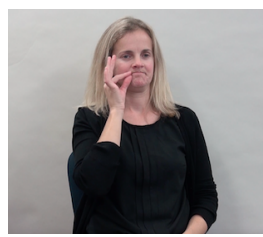

DON'T-TELL

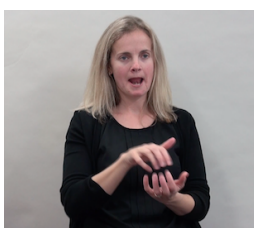

EAT-MY-WORDS

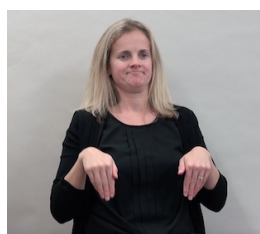

BE-PASSIVE

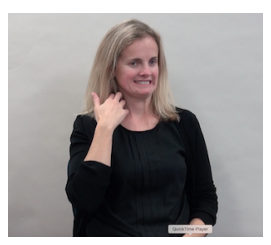

SELF-CONSCIOUS

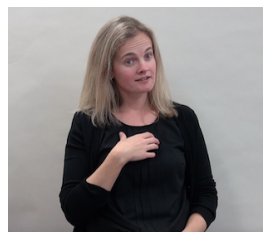

GOSH

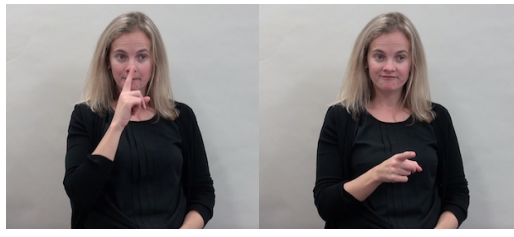

UNEXPECTED 


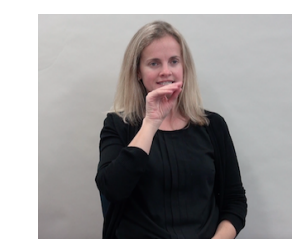

SPEAK

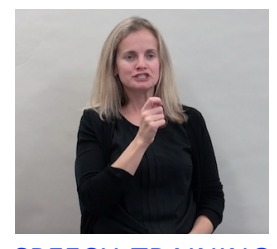

SPEECH-TRAINING

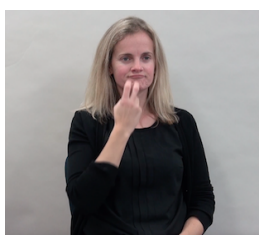

ORAL / SPEAKING

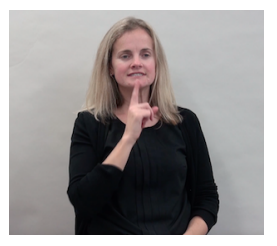

SAY 2

Figure 6.2: NZSL folk definitions using synonymy

The data showed that synonymy was frequently used to identify different senses of polysemous signs of different word classes. For example, responses to target sign $\mathrm{AC}_{5}$ included SAD (http://nzsl.vuw.ac.nz/signs/4435), CALM / PLAIN (http://nzsl.vuw.ac.nz/signs/ 666), and PRIVATE (http://nzsl.vuw.ac.nz/signs/5402 in its two-handed form). Each of these alternatives have a more restricted meaning than the polysemous target sign itself, but taken together they show the possible senses of a sign in a succinct way.

For target sign CC1 ASK / REMIND / TELL, synonyms covered these three senses and the additional underlying sense of getting someone's attention. Two of the synonyms refer to other culturally appropriate / culture-specific ways of getting attention in the Deaf community: by waving a hand in their line of sight, or by stomping on the floor.

Folk definitions for sign CC $_{5}$ DEVELOP / GROW did not include synonyms for its core sense of physical growth of plants, but the figurative extension of this sense to refer to other improvement, advancement or development was conveyed by synonyms such as IMPROVE and MOVE FORWARD.

As was discussed in section Extension and comprehension (under section 5.2.5.1), sign BC 5 SMALL-ROUND-FOOD-PLURAL is a partly lexicalised polycomponential form in which the handshape represents small, round things, most typically edible things. Polycomponential signs were commonly used as generic hypernyms in NZSL, with different senses being specified by different accompanying English mouth patterns. McKee (2007) mentioned as an example the polycomponential sign ROOF with senses 'house', 'home', and 'factory'. Over time, more specific signs have developed in some areas, and while the individual meaningful components of the generic sign remain available for incorporation into newly coined forms, the extension of the lexicalised form may narrow. Since the participant group in the current study comprised people of different ages, the number of synonyms recognised or used by an individual participant offers a snapshot view of changes over time as new (often non-native) 
signs enter the lexicon. For example, younger participants, with one exception, indicated that they never used sign $\mathrm{BC}_{5}$ to refer to 'peas' or 'berries', instead using the signs PEA and BERRY / STRAWBERRY; whereas some of the older participants remembered using sign $\mathrm{BC}_{5}$ for these senses in the past, or still used it. Although multiple referents are still possible for sign $\mathrm{BC}_{5}$, the prototypical referent in the data was 'grapes'. Only four participants provided a synonym for this sense, and this possible alternative form (depicting a bunch of grapes dangling from a vine) showed considerable variation in production, suggesting that it has not yet been fully lexicalised. Some other synonyms were also alternative polycomponential forms using the same handshape but with a different movement: for example, OLIVES used two hands in alternate movements as if placing olives on a pizza.

The high level of lexical variation in NZSL noted in section 2.1.5 - Sociolinguistic variation was also evident in the folk definitions for sign $\mathrm{CP}_{5}$ HEARING, which included two older variants, one variant said to have been introduced via Australasian Signed English, and one recent ASL sign that is recognised but not used widely in NZSL. The synonym LESS is a nonnative lexical equivalent for sign $\mathrm{DP}_{5}$ DECREASE and incorporates a fingerspelled $\mathrm{L}$ handshape (see section 2.2.5.1 - Fingerspelling). Sign EP2 SPEECH is itself an older sign which was no longer actively used by any of the participants; the synonyms provided in this case showed current variants, and also reinforced that many Deaf people associate 'speech' with speech training and oral education methods (see section 2.1.4 - Language contact between signed and spoken languages).

Idiomatic signs such as AC4 KEEP-INSIDE-ONESELF and EC4 I-WAS-WRONG / YOU-GOT-ME were found to be the most frequently defined by synonymy. The synonyms were themselves idiomatic but called on different underlying metaphors: for example, while KEEP-INSIDE-ONESELF refers to emotions that are 'held' in the chest, KEEP MUM (http://nzsl.vuw.ac.nz/signs/4029) uses the metaphor of holding spoken words in, and PUT-UP-WITH (http://nzsl.vuw.ac.nz/signs/1569) shows emotions being suppressed.

Many participants initially suggested the phrase STRONGLY/VERY DEAF as a synonym for sign $\mathrm{BP}_{5}$ DEAF (when used in its sense as an identity label rather than an audiological distinction), but then proceeded to compare the two terms in more detail and found that they were different, although the difference could not be easily expressed. The intensifier STRONGLY/VERY (http:// nzsl.vuw.ac.nz/signs/3266) corresponds with the intensifier that is part of $\mathrm{BP}_{5}$ ( a sign phrase consisting of the signs FULLY and DEAF); another synonym also included an intensifying aspect 
by producing the sign DEAF with a slow, emphatic movement. The phrase BIG-D DEAF was suggested by three participants. As teachers of NZSL who had undertaken some formal study of sign language linguistics and Deaf studies, these participants were familiar with the convention of writing the word Deaf with a capital D to indicate Deaf people as a cultural and linguistic community. The sign phrase BIG-D DEAF may be confined to a more academic register.

The occurrence of synonyms NEXT-TOPIC and NEXT (with mouth pattern 'shift') for sign AP1 PUT-ASIDE suggests that functional equivalence takes precedence over semantic content in the explanations for signs such as these which have a discourse coordination function.

\subsubsection{Exemplification}

In exemplification, as the category name implies, an example is given of something that characteristically possesses the word being defined as an attribute, or performs it as an action. Casagrande and Hale (1967) identified exemplification as the reverse of attribution: whereas in attribution, a subject is defined by its predicate (its attributes), in exemplification the predicate is defined by the subjects it can take. In line with this characterisation, exemplification in the NZSL data was used to describe signs that can function as predicates, including verbs (e.g. DECREASE, GROW, KEEP-INSIDE-ONESELF) and adjective-like signs (e.g. PLAIN, DEAF). The predominance of signs belonging to these word classes in my data may have led to the more frequent use of exemplification when compared to Casagrande and Hale's study, which included a large number of nouns. In my data, 10 of the 15 target signs were defined using exemplification.

Figure 6.3 shows typical instances of signed folk definitions that made use of exemplification. 


\section{AC4 KEEP-INSIDE-ONESELF}

LIKE ONE WAY I HAVE PRIVATE SOME THING KEEP-INSIDE-ONESELF OR I ANNOYED HOLD-INSIDE KEEP-INSIDE-ONESELF [...] LIKE FEEL-HURT KEEP-INSIDE-ONESELF GRIEF FEELING MORE FEELING YES

One way (this sign can be used) is that I have something that I keep private, that I keep to myself; or I'm feeling annoyed and I'm bottling it up [...] It's like keeping feelings of being hurt or grief inside you; It's more feelings, yeah.

AC5 PLAIN / CONFIDENTIAL, PRIVATE

PLAIN FLAVOUR [...] PLAIN COLOUR [...] PLAIN FACIAL-EXPRESSION CAN

plain flavour[ ...]plain colour [...] It can be (used for) plain facial expression

\section{BC2 UNINTELLIGIBLE-VOICE}

MAN UNINTELLIGIBLE-VOICE HE LIKE ROUGH, ROUGH UNINTELLIGIBLE-VOICE IT HE MAN CL:PERSON TEND-TO

It tends to be men who have an unintelligible voice, like a rough voice quality.

\section{BP5 DEAF}

LIKE ME DEAF ME [...] FOR-EXAMPLE (SIGN NAME) DEAF HE/SHE [...] FATHER DEAF HE

Like me, I'm culturally Deaf [...] For example, (person in the Deaf community) is culturally Deaf [...] My father is culturally Deaf.

\section{CC1 ASK / REMIND / TELL}

CAN ONE-TO-ONE CAN BOSS FORMAL INFORMAL ANYTHING DOCTOR PLEASE ASK DOCTOR [...] YOU ASK SHE

You can (use this sign with) one person, it can be your boss; in formal or informal situations, anywhere: (it can be) the doctor: "please ask the doctor".[...] You ask her.

\section{CC5 DEVELOP /GROW}

LEARN GOOD ENGLISH DEVELOP LEARN MORE THING

(I'm) learning good English (skills), they're developing, I'm learning more things.

FLOWER GROW

Flowers grow.

\section{DC1 TALK-IN-SIGN-LANGUAGE}

DEAF TALK-IN-SIGN-LANGUAGE HEARING TALK-TO-EACH-OTHER

Deaf people chat with their hands, and hearing people chat with their mouths.

\section{DC3 BEEN-TO / HAVE-EXPERIENCE-OF}

I BEEN-TO I FLY-TO AUCKLAND BEEN-TO FINALLY

I've been to Auckland by plane, finally!

I RUGBY PLAY I HAVE-A-LOOK HAVE-EXPERIENCE-OF IT

I've given rugby a try-l've done that.

\section{DP5 DECREASE}

\section{NUMBER DECREASE}

Decreasing in number.

\section{EC1 ONE-HANDED-FINGERSPELLING}

DEAF VISIT HE/SHE ONE-HANDED-FINGERSPELLING CAN'T-UNDERSTAND

The Deaf visitor used one-handed fingerspelling-I couldn't understand it at all.

Figure 6.3: NZSL folk definitions using exemplification 
From these examples it can be seen that many exemplifications were abstracted larger classes of objects or people: e.g. 'feelings', 'men', 'colours', 'numbers'. Other exemplifications were typical instances drawn from personal experience: e.g. having been to 'Auckland' (rather than 'a place'), or having tried out 'rugby' (rather than 'a sport'). Culture-bound signs such as DEAF were related to participants' own identity and that of individuals in the surrounding community.

Exemplification was always used in conjunction with other semantic categories rather than in isolation. The extracts in figure 6.3 show how exemplification was combined with synonymy (HAVE-A-LOOK / TRY as a synonym for BEEN-TO / HAVE-EXPERIENCE-OF; FEEL-HURT as a synonym for KEEP-INSIDE-ONESELF) and attribution (ROUGH as an attribute of UNINTELLIGIBLE-VOICE). Many participants used extensive exemplification as part of a comparison between two or more signs that have similar or identical senses, but that differ in semantic and colligational restrictions on the subjects they select. Figure 6.4 shows the target signs DECREASE, GROW, BEENTO / HAVE-EXPERIENCE-OF, and TALK-IN-SIGN-LANGUAGE and the signs that they were contrasted with through listing different exemplifications.

In some cases, exemplification revealed clear contrasts, such as the sign TALK IN SIGN LANGUAGE being exemplified by DEAF, and SPEAK (in a two-handed form to indicate two speakers) by HEARING: i.e. whereas hearing people talk with their mouths, Deaf people talk with their hands. In other cases, there were more vague boundaries between the subjects that could be selected. For example, the prototypical exemplification of the sign BEEN-TO / HAVE-EXPERIENCEOF is a country or a place, whereas HAVE-DONE-THAT was usually exemplified by a new experience or activity, for example seeing a movie or trying out a new restaurant; in between these prototypical exemplifications there were a number of experiences or activities for which either sign could be used (for example, trying out a new sport or bungy jumping). Preferences for one of the signs or the other of these signs (both relatively recent borrowings from other sign languages) varied between participants. 


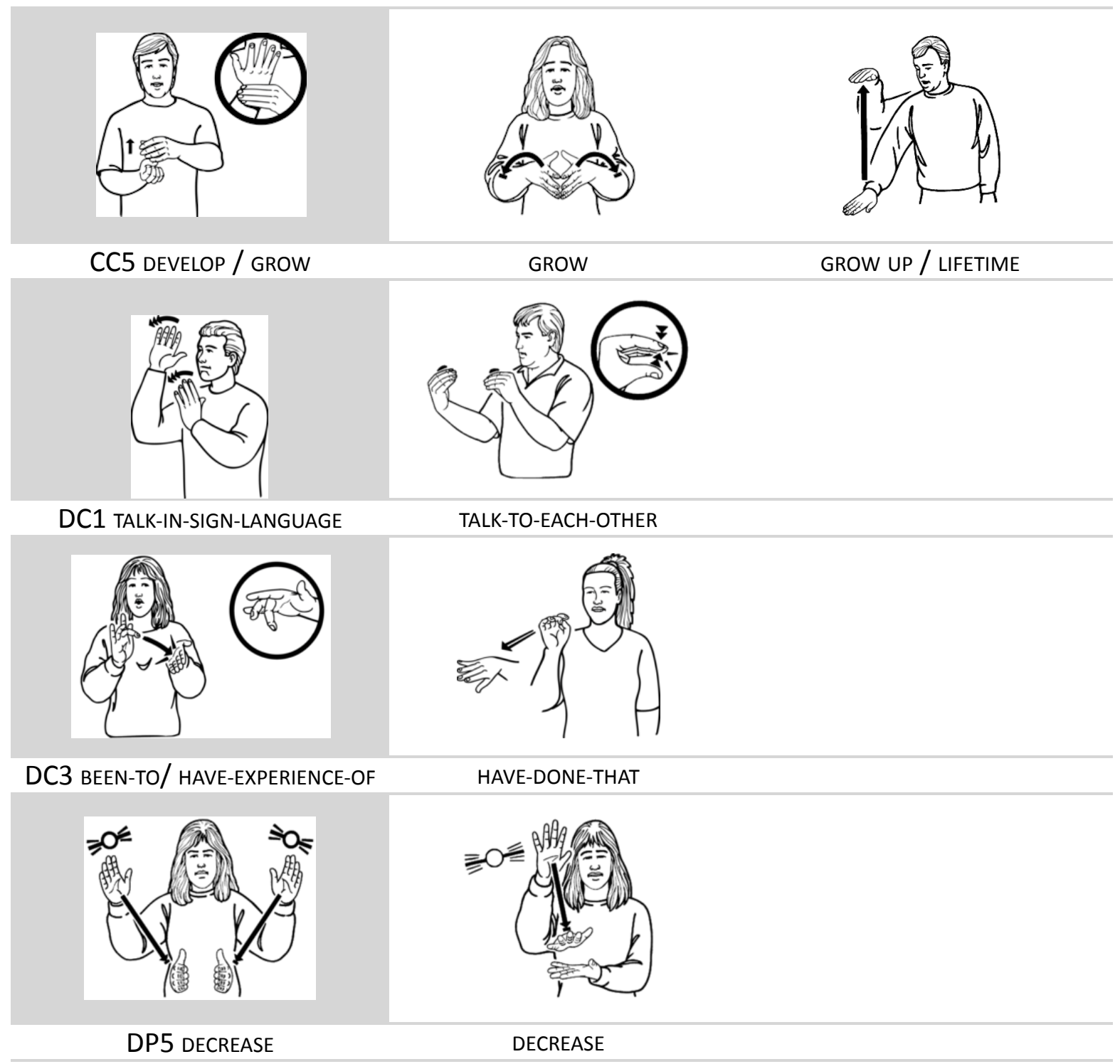

Figure 6.4: Comparison of related signs through exemplification

To illustrate further how even a small sample of exemplification in folk definitions can provide detailed information, figure 6.5 shows the range of examples given during the comparison of the signs labelled here as DECREASE 1 and DECREASE 2. 


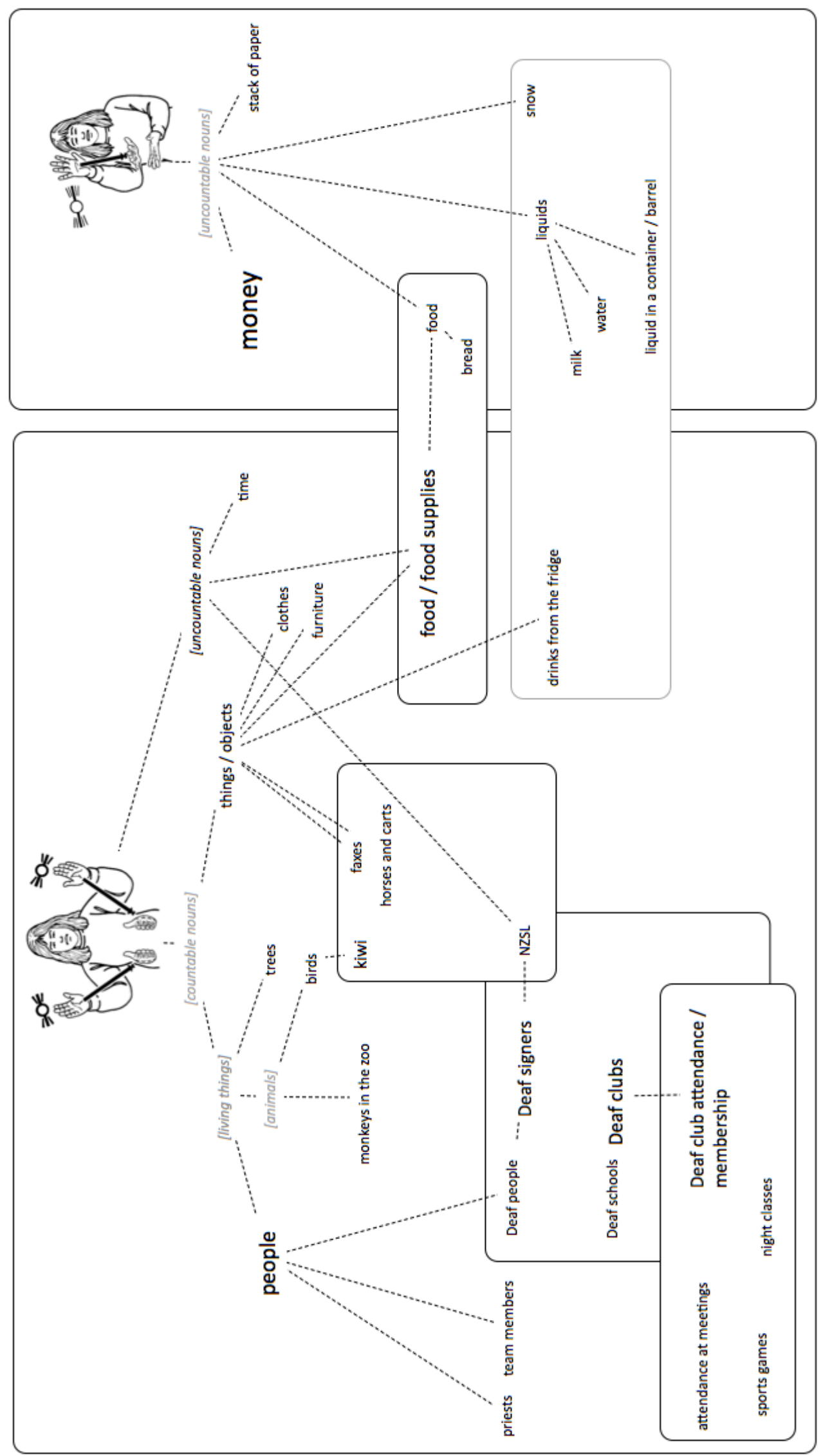

Figure 6.5: Selectional restrictions for two signs: DECREASE 1 and DECREASE 2 
First of all, prototypical examples can be pinpointed by comparing how often the same example is given by multiple participants. The size of the glosses in figure 6.5 shows their relative frequency of occurrence in the data: for example, the sign MONEY was mentioned by 9 participants, whereas the sign STACK-OF-PAPER was mentioned only once. Even with a small participant group, repeated mentions are a good indication that MONEY is a more typical example.

Secondly, colligational restrictions can be explored by noting that examples for DECREASE 1 are mainly countable, whereas examples for DECREASE 2 are uncountable. The category of 'food and drink' appeared as an example for both DECREASE 1 and DECREASE 2. Mostly, these examples maintained the distinction between countable items (e.g. drinks from the fridge) and uncountable mass nouns (e.g. MILK, FOOD). However, there were also examples of DECREASE 1 being used to talk about food becoming scarce. A few other uncountable nouns (TIME and NZSL) were also given as examples of DECREASE 1 , suggesting that there is also an overriding semantic distinction between the two signs, with DECREASE 1 referring to a dwindling in numbers to the point of something becoming scarce or rarely seen, and DECREASE 2 referring to situations where items can be depleted altogether.

Thirdly, examining the connections between individual signs given as examples reveals overlapping networks of similarities. For example, hierarchical semantic relationships can be identified: MILK and WATER can be grouped together under the superordinate 'liquid' (expressed differently by several participants in the absence of a conventional sign); PRIEST, TEAM MEMBER, and DEAF PEOPLE all fall under the superordinate sign PEOPLE. In other cases, no superordinate sign was provided but hierarchical relationships can still be postulated (shown as [category] in the figure): for example, birds and monkeys are instances of animals, which together with people and trees can be categorised as living things. Other examples are related non-hierarchically, such as 'attendance at meetings', 'Deaf club attendance', 'night classes', and 'sports games' all being gatherings where the number of people in attendance may increase or decrease.

Finally, the large cluster of signs relating to the Deaf community demonstrates that specific cultural 'frames' (Fillmore, 2003) indeed have a prominent place in Deaf people's folk definitions, even when the sign itself does not have a specific cultural meaning or connotation. In the case of DECREASE 1, the large number of Deaf community-related examples most probably stems from a connotation with previously plentiful objects, animals or 
resources decreasing to the point of extreme scarcity or extinction. There is therefore a connection between examples such as kiwi (a bird threatened with extinction), horses and carts and faxes (both almost obsolete in modern day society), and the concerns Deaf people have about the closure of Deaf-specific institutions such as Deaf schools and Deaf clubs, and the impact this has on the future of sign languages.

\subsubsection{Comparison}

Comparison involves describing a word in terms of its similarity or contrast with another word. In Casagrande and Hale's (1967) data, comparisons were often introduced with phrases such as 'it is like / looks like ...' and followed by a number of attributes showing how the two items differ. In my data, target sign EC1 ONE-HANDED-FINGERSPELLING was said to be like the sign FINGERSPELL / SPELL (http://nzsl.vuw.ac.nz/signs/4127), but performed with one hand-an attribute that distinguishes it from the two-handed manual alphabet. Several participants also listed the countries in which each type of fingerspelling is used, typically contrasting England, Australia and New Zealand's use of a two-handed alphabet with America and Europe's use of one-handed alphabets. As well as having an attributive part, this comparison was therefore also the only instance of the provenience category (where something is defined by its source) in the data.

Casagrande and Hale noted that comparisons took a range of grammatical forms that were not always signalled with 'is like / looks like'. The previously mentioned comparisons between signs through contrasting exemplification (see figure 6.4) were not overtly introduced as a comparison by the participants, but instead used implicit strategies such as apposition and parallelism (Chaudron, 1982).

Participants expressed uncertainty about the precise difference between the sign phrases FULLY DEAF and STRONGLY/VERY DEAF. Figure 6.6 shows translated extracts of three participants' comparisons of these two signs.

From these different explanations it can be seen that sign $\mathrm{BP}_{5}$ (FULLY) DEAF can be used either to refer to a person's level of sound perception (i.e. they are totally deaf and cannot hear anything at all), or to a person's identity as a Deaf person. When used in this second sense, it is near-synonymous to the sign STRONGLY/VERY DEAF, which is also used to refer to the cultural identity of Deaf people. 


\section{Participant 1:}

I feel that 'fully Deaf' (is used) if you're talking about audiology, but if it's about behaviour, I (use) 'strongly / very Deaf' and 'characteristic / typical'.

\section{Participant 2:}

Fully Deaf, well, it can be a description, so you talk about (level of) deafness, as in 'he is totally Deaf'; or about experience, he has a fully Deaf identity. It can be either. [...] 'Fully Deaf', if (you're asked if someone is a little Deaf, you would respond) no, 'totally Deaf', it's like a comparison. 'Strongly Deaf' is the same, it's a comparison, but it's maybe different; if you're comparing two people and the first person is strongly Deaf and the other is strongly hearing. It can be (used in) both ways, but it includes identity too, as in this person is strongly hearing, and the other is strongly Deaf, kind of like that. Or it could be that the sign 'fully' has more English influence. I don't know.

\section{Participant 3:}

Yes, I have used (that sign) many times. I say my family are fully Deaf, strongly Deaf.... there is a small difference, although they're almost the same (signs). Fully Deaf, like I said before, (means) you are proud to be Deaf and everything Deaf-related, but strongly Deaf means ... oh they are very similar ... strongly, I think I know, it means if a family is strongly Deaf, they are all fluent signers, the family don't use speech at all, they use captions (on the TV), they use captions a lot

Figure 6.6: Comparison between the sign phrases FULLY DEAF and STRONGLY/VERY DEAF

While participant 1 recognised that FULLY DEAF was used by some people in this second sense, they themselves exclusively used STRONGLY / VERY DEAF. Participant 2 used either sign but suggested that perhaps the term FULLY DEAF was a sign variant based on a transliteration of the English phrase 'totally Deaf'. The explanation by participant 3 (and other folk definitions not shown here) seems to suggest that there is a subtle difference between the two signs. FULLY DEAF is used as an identity label to refer to people who are completely immersed in the Deaf community, whereas STRONGLY/VERY DEAF is used when the focus is on behaviours and attitudes typical of a Deaf person in the context of the Deaf community (even though they may behave differently in other contexts).

\subsubsection{Attributive}

In attributive definitions, the item is defined by listing its properties, distinctive markers or typical behaviours. The attributive category mainly contains definitions of nouns and was the most used category in Casagrande and Hale's (1967) study, including most definitions of animals, plants, and kinship terms. As was mentioned before, the target signs in my study included few nouns. This may be one reason why the attributive category was used less often in my data. It is also likely that the nature of the signed lexicon influenced the less frequent 
use of this category. In the extremely productive class of polycomponential signs (see 2.2.4.1 - Depicting signs or polycomponential signs), individually meaningful components such as the handshape, location and movement of the sign depict attributes such as size, distinctive markers, or behaviours as a part of the sign. Table 6.4 shows how attributive properties found in Casagrande and Hale's Papago folk definitions are already inherent in the NZSL signs for these referents.

\begin{tabular}{ll}
\hline Referent & Attributes described in Papago folk definitions \\
(Casagrande and Hale, 1967) & $\begin{array}{l}\text { it has a long tail (...) but it has a stinger standing on its tail; } \\
\text { with which it bites or stings people } \\
\text { scorpion }\end{array}$ \\
grasshopper & $\begin{array}{l}\text { another thing goes around through the grass; it will jump } \\
\text { so far and then land; then forward again }\end{array}$ \\
than which goes over and thereby gives us light &
\end{tabular}

Table 6.4: Attributive folk definitions compared with attributive features inherent in NZSL signs

Some folk definitions in my data did emphasise or paraphrase the inherent attributive components of the sign: for example, by describing sign EC1 ONE-HANDED-FINGERSPELLING explicitly as being produced with one hand, or by isolating the handshape of sign $\mathrm{BC}_{5}$ SMALLROUND-FOOD-PLURAL, holding it static and mouthing the English words 'small' and 'round' while tracing the circular handshape with the index finger of the other hand.

In other folk definitions, production of the target sign was modified to focus on particular attributes. For example, target sign BC2 UNINTELLIGIBLE-VOICE itself was repeated with a more erratic movement to show uncontrolled voice production.

Some attributive signs were also modified to correspond with features of the target sign they were describing. For example, the sign DEEP (http://nzsl.vuw.ac.nz/signs/5873) was produced 
in the location of target sign AC1 KEEP-INSIDE-ONESELF; and the sign EMPTY (http://

nzsl.vuw.ac.nz/signs/4801) was produced with a downward movement down the upper body to show that PLAIN clothing is devoid of any patterns.

More than half of the participants included the sign FOR-AGES (http://nzsl.vuw.ac.nz/signs/ 2430) as an attribute of TALK-IN-SIGN-LANGUAGE, reflecting Stock's (1988) observation that folk definitions include subjective contextual information as well as objective characteristics.

\subsubsection{Antonymy}

In the antonymy category, a word or sign is defined as the opposite or negation of another item. Instances of antonymy in my data are shown in figure 6.7. These examples correspond with Casagrande and Hale's (1967) finding that antonymy was commonly used to define adjectives.

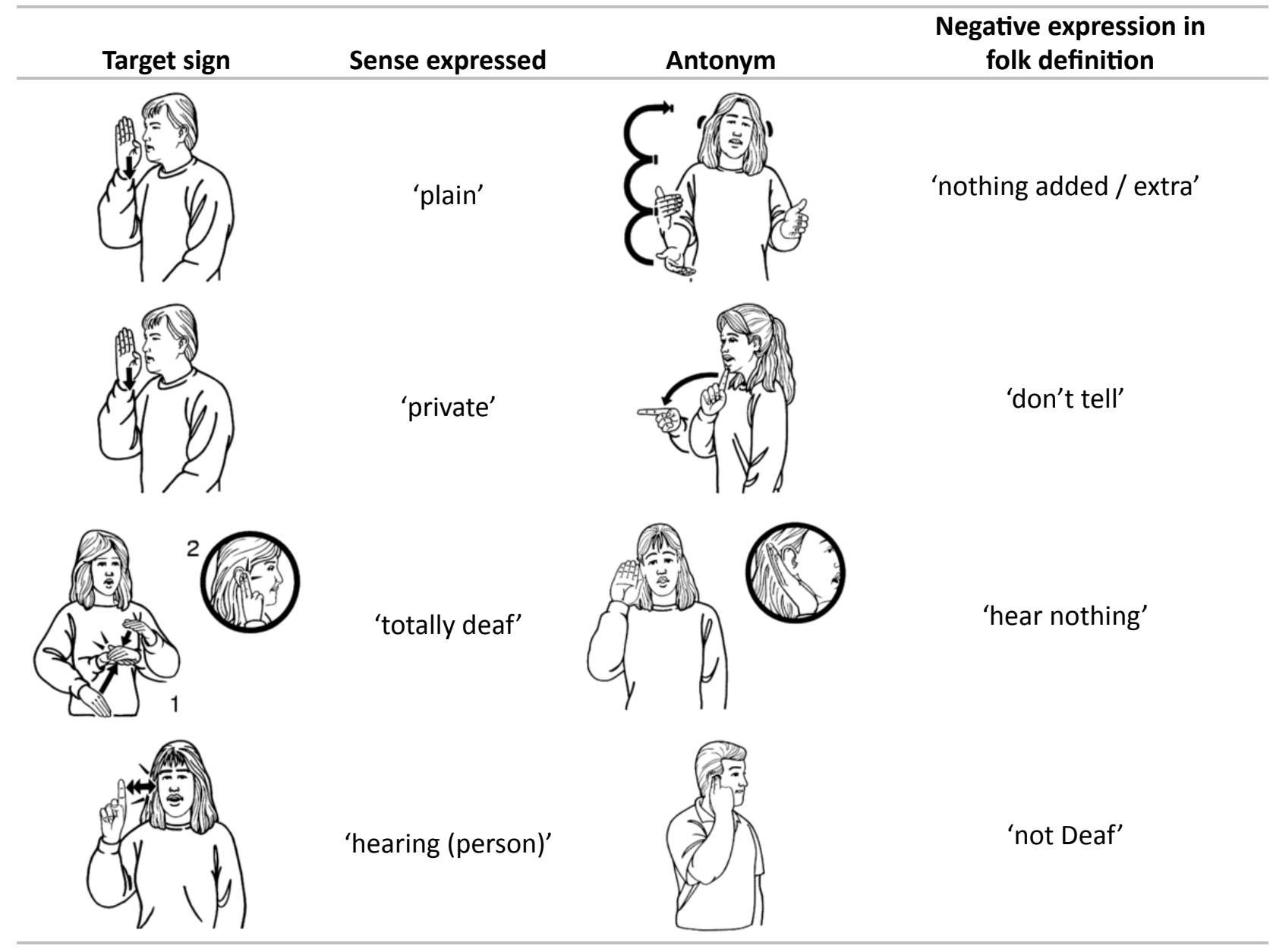

Figure 6.7: NZSL folk definitions using antonymy 


\subsubsection{Other semantic categories}

Only isolated occurrences of the remaining semantic categories were found in the NZSL folk definition data. The reasons noted for the less frequent use of attributive definitions also apply to other categories. The fact that most of the target signs were verbal or adjectival in nature meant that categories that mostly pertain to nouns were less frequently used. Also, polycomponential depicting signs not only include information about the size or shape of objects (attributive), but also about how an object is handled (operational), what it is used for (function), and the object's location in relation to other objects (spatial). For example, in sign CC1 DEVELOP/GROW, the non-dominant hand fulfils the function of a locative depicting the ground, from which a plant is shown to emerge and grow upwards through the movement of the dominant hand. Attention can be drawn to these inherent iconic properties, for example by producing the movement of a sign slowly and emphatically, by holding the handshape and pointing to it with the other hand, or by pointing and directing eye gaze to the location of the sign. However, most of the time these characteristics were not remarked upon by participants, who instead focused on other strategies such as synonymy and exemplification.

Class inclusion, where the folk definition states that ' $\mathrm{x}$ is a y' (e.g. 'a pigeon is a bird'), was only used to indicate very general classes using signs such as THING (http://nzsl.vuw.ac.nz/signs/ 6169), SOMETHING (http://nzsl.vuw.ac.nz/signs/4326), or PERSON (http://nzsl.vuw.ac.nz/signs/ 4446): for example, CP5 HEARING was described as “HEARING G:WELL HEARING PERSON”; and BC5 SMALL-ROUND-FOOD-PLURAL was described as "SOMETHING YOU EAT". Chaudron (1982) referred to these general classes as 'lexical cover terms' and found that they were used for the purpose of referential cohesion rather than classical definition. Class inclusion is another definition category that is likely to be seen as redundant by Deaf participants, since many polycomponential signs include what has been called a 'classifier' handshape (see section 2.2.4.1 - Depicting signs or polycomponential signs) that identifies the sign as belonging to a particular class. In the case of $\mathrm{BC}_{5}$ (small round) food (plural), therefore, while some participants identified FOOD as the most typical class referred to by this sign, the wider class of small, round things (including, for example, stones and polka dots on a dress) was not explicitly stated by many participants.

Finally, circular definitions were given by several participants when asked to define sign $\mathrm{CP}_{5}$ HEARING. Translated into English, circular statements about this sign included: 
- $\quad$ hearing, well, it's hearing

- hearing, well, it's a hearing person

- a person who is hearing

- things that are typically hearing, hearing behaviours

Statements like these may indicate that the participants struggled to find an adequate description, or that the meaning of the sign was so obvious to them that they did not see a need to define it.

\subsubsection{Other characteristics}

As was described in the literature review, the potential usefulness of folk definitions as a model for dictionary definitions extends beyond the way in which semantic content is conveyed. Stock (1988) argued that whereas in dictionaries, explanations of word meaning and other information about the word, such as register, typical contexts and grammar are treated in different parts of the entry, folk definitions incorporate contextual information about a word's use as an inseparable aspect of its meaning. This property of folk definitions proved to be true for my data as well. Information about the register, variation, and grammatical characteristics of the target signs was found both in the contexts embedded in the NZSL folk definitions through exemplification, attribution, and comparison; and in comments about the situations in which the participants themselves or others would use these signs.

\subsubsection{Register and situations of use}

Indications about register and situations of use were most evident in the folk definitions of target signs belonging to the semantic field of communication, such as CC1 ASK/REMIND/TELL and DC1 TALK-IN-SIGN-LANGUAGE. There were several different ways in which register was referred to. Firstly, participant used lexical labels for particular registers, for example FORMAL, INFORMAL, SOCIAL, RELAX ('casual') and STORY ('narrative') (see figure 6.8).

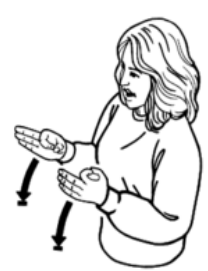

FORMAL

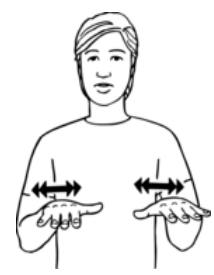

INFORMAL

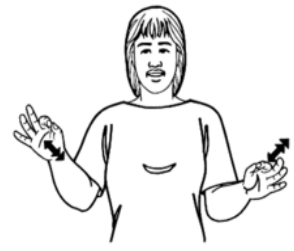

SOCIAL

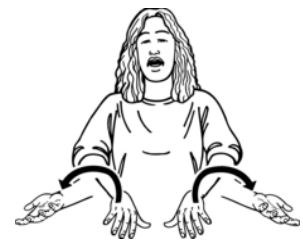

RELAX(ED)

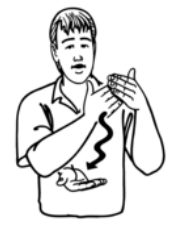

STORY

Figure 6.8: Signs used in the NZSL folk definitions to indicate register or situations of use 
Register or situations of use were implicit in the typical contexts referred to by participants in the folk definitions. Some instances of exemplification (see figure 6.3), for example, identified the register of the target sign by specifying the people involved in a situation where the sign would be used (e.g. target sign CC1 ASK/REMIND/TELL used with your boss or a doctor). Attributes such as the previously noted association of DC1 TALK-IN-SIGN-LANGUAGE with FORAGES also provide register clues. Again, there were extended comparisons of signs with a similar semantic content but belonging to different registers or situations of use. Table 6.5 shows how differences in field, tenor, and mode (Halliday \& Hasan, 1976 ) of these contexts can be used to compare signs brought up by the participants in response to target sign $\mathrm{DC}_{1}$ TALK-IN-SIGN-LANGUAGE.

The main distinction in field was between situations requiring serious, in-depth, regulated discussion and social situations where topics of discussion can range widely. In terms of tenor, the most salient distinctions were between one-to-one meetings and groups, and between hearing people and Deaf people (or other fluent sign language users). Some signs, such as TALK-IN-SIGN-LANGUAGE and TALK-TO-EACH-OTHER were mainly distinct in mode, whether signing or speaking (and, in one case, writing). When no particular situations were specified, for example in the case of TALK (http://nzsl.vuw.ac.nz/signs/2466), we can assume that this sign was less strongly associated with a particular register. 


\begin{tabular}{lllll}
\hline Sign & Situation & Field & Tenor & Mode \\
\hline MEET & DIFFERENT TOPIC & DEAF & SIGN \\
CATCH-UP & DIFFERENT ISSUE & FRIEND & \\
DEAF-CLUB & ANYTHING & GROUP & \\
MIX/SOCIALISE & OFF-TOPIC & INTERPRETER & \\
COFFEE & & & \\
NIGHT & & &
\end{tabular}

DC1 TALK-IN-SIGN-LANGUAGE

http://nzsl.vuw.ac.nz/signs/1300

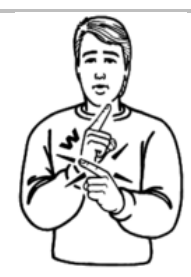

TALK

http://nzsl.vuw.ac.nz/signs/2466

$\begin{array}{lll}\text { MEETING } & \text { SERIOUS } & \text { GROUP } \\ & \text { INDEPTH } & \text { ONE-TO-ONE } \\ & \text { TO-THE-POINT }\end{array}$

DISCUSS

http://nzsl.vuw.ac.nz/signs/1211

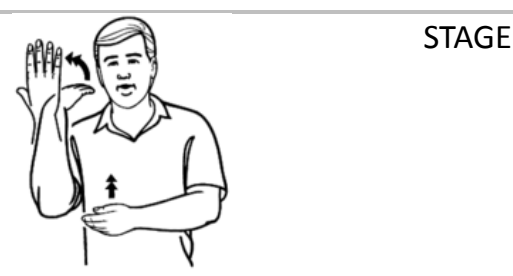

AUDIENCE

MAKE-A-SPEECH

http://nzsl.vuw.ac.nz/signs/972

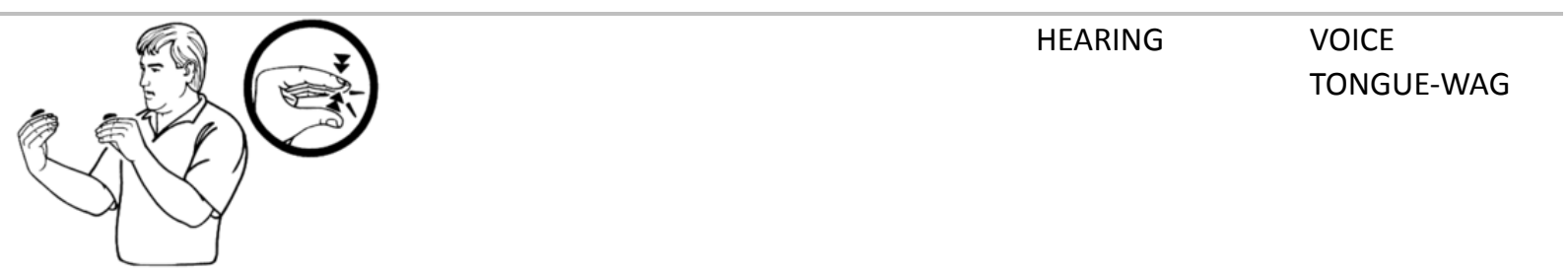

TALK-TO-EACH-OTHER

http://nzsl.vuw.ac.nz/signs/4301

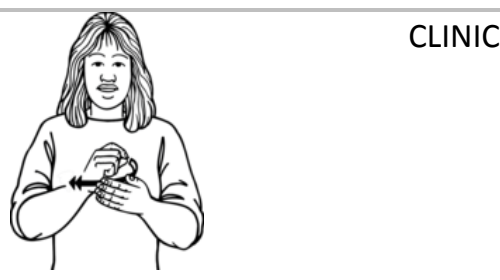

DOCTOR WRITE

(implied:

'hearing

person')

WRITE-NOTES-BACK-AND-FORTH

Table 6.5: A comparison of the register of signs related to 'talk' through the contexts referred to in NZSL folk definitions 
Figure 6.9 shows examples of comments about signs AP1 PUT-ASIDE and CC1 ASK/REMIND/TELL. These comments (shown here translated into English) revealed participants' metalinguistic awareness of differences in their own and other people's usage of these signs in different situations.

\begin{abstract}
AP1 PUT-ASIDE
It's like when me and (name of a person) are at work and I'm going into detail (about a topic), then I would say "no, we'll put that aside and get on with work." After we finish work, we can put that aside and then it's ok to have a social chat.

It can be used when there's an audience-it's used in one-to-one situations but it can be used with a group as well. Maybe at an AGM when someone is presenting in detail and they will say "we'll put that aside and move on to the next point and address that briefly"; or at a board meeting, moving on to other points.

\section{CC1 ASK/REMIND/TELL}

(I use this sign) in conversation, more like at work or at school. I don't use it much in my home environment-there might be the odd time, yes, but I don't use it much when I'm having a normal chat in a group about all sorts of things. But if I'm specifically talking about rugby or work, or if I'm saying "I'm reminding you about our appointment"-when it's more important to know the exact time, $i$ kind of use it like that.
\end{abstract}

Figure 6.9: Comments about register in NZSL folk definitions

Participants also demonstrated how the production of the target signs could be affected by register. Figure 6.10 shows two variant forms of target sign AP1 PUT-ASIDE. Participants indicated that in formal situations, they would use a two-handed form, whereas a onehanded form with a more relaxed movement would be more typical in informal interactions.

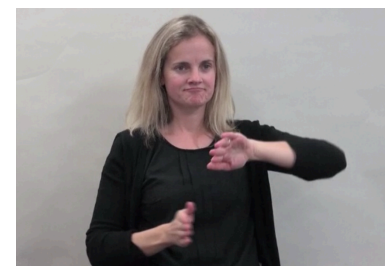

PUT-ASIDE

(formal, 2-handed)

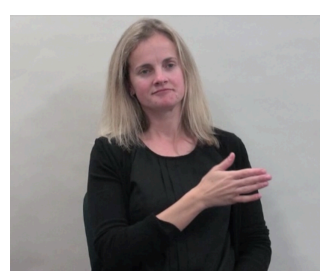

PUT-ASIDE

(informal, 1-handed)

Figure 6.10: Two variant forms of the sign PUT-ASIDE in formal and informal production

Some comments highlighted cultural norms around the appropriate use of certain signs. For example, participants stated that sign BC2 UNINTELLIGIBLE-VOICE was acceptable as a description of a person in a narrative but not when describing someone who is present or 
when addressing someone directly in a conversation, except as a joke between Deaf people. Similarly, several participants remarked that they would never use sign $\mathrm{BP}_{5}$ DEAF when asking another person about their status in terms of hearing loss or community membership. Such a question could be considered rude. Instead, the sign was mostly used to assert one's own status in response to a perceived challenge.

A final observation made by several participants was that idiomatic signs belonging to an informal register, such as EC4 I-WAS-WRONG/YOU-GOT-ME, were often used incorrectly by hearing learners of NZSL. The overuse of such signs by hearing learners, including sign language interpreters (as advanced non-native learners), tended to result in constructions that were either grammatically or semantically incorrect, or not appropriate for the register of the conversation.

\subsubsection{Variation}

Comments about variation in the folk definitions (see figure 6.11) highlighted the sociolinguistic variables that are salient to Deaf NZSL users. Both age and region were frequently mentioned in relation to the target sign, often in conjunction with a synonym for the target sign used by another age group or region. In addition to these internal sociolinguistic variables, the external influence of English-based sign variants (see section 2.1.4 - Language contact between signed and spoken languages), was recognised by participants who labelled some signs as ENGLISH INFLUENCE or T-C- an abbreviation for 'Total Communication', an approach that became prevalent in New Zealand in the 1980s (see e.g. Ahlgren, 1986). The Total Communication approach encouraged the use of both signing and speaking and saw the introduction of a large number of signs that did not originate from the New Zealand Deaf community.

In the user behaviour study reported on in section 4.6.4.2 - Making sense of search results, it was found that the absence of variation information made it difficult for learner users of the ODNZSL to choose an appropriate sign variant from the search results. Including such information in the dictionary would therefore be beneficial. However, it was also noted that reliable information on variation is not always available to the dictionary compilers. Unfortunately, subjective comments made by the participants as part of the folk definitions were an inexact source of information about variation. While some variants were labelled absolutely (e.g. CHRISTCHURCH), other sociolinguistic labels were interpreted in relation to the participants themselves. The sign YOUNGER, for example, was seen as referring to children and 
adolescents by a participant in their late 2os, whereas a participant in their 6os saw the YOUNGER age group as people below the age of 30. Similarly, regional labels such as NORTH were open to interpretation depending on the location where the interview took place and the participant's own place of residence. Participants' own sociolinguistic backgrounds were also varied: for example, they may have attended several schools, moved between regions or spent considerable time abroad or in contact with users of other sign languages. Participants' perceptions about signs being in use in other regions were therefore not always consistent with data collected in these regions.

\section{CC1 ASK / REMIND / TELL}

No, I don't use ASK-2, I use ASK-1. I get quite frustrated because the class (that I teach) don't use ASK-1, they keep using ASK-2. And I'm thinking why don't students like to use ASK-1? Well, if you use ASK-1 it's nice, it's more strongly a Deaf sign. It's a nice sign, it shows how you use your hand to get attention. If you use ASK-2, it shows a strong English influence, but ASK-1 has no English influence at all. You can use ASK-1 to show different inflections for person and it's a nice use of space. But if ASK-2 is inflected, it uses space in a limited way.

\section{DC3 BEEN-TO / HAVE-EXPERIENCE-OF}

Really, (the sign) BEEN-TO is not in my vocabulary. I only use FINISHED. It's not in my vocabulary but I have seen people use BEEN-TO. I'm trying to think how to describe the people who use that sign-maybe late signers, people who acquired sign language later in life, like they started to learn sign language when at school, or they used TC and only changed to using NZSL later. You won't see older people use it in conversation. No, I don't think it's older people, it's late signers who use BEEN-TO. When I talk about 'late signers' I also mean overseas people who may have moved to New Zealand and acquired New Zealand sign language later. My parents use FINISHED, not BEEN-TO.

\section{EC4 I-WAS-WRONG / YOU-GOT-ME}

I think it's more used in the North, they sign I-WAS-WRONG. [...] The sign SELF-CONSCIOUS is slowly disappearing, I think, but they're still using it in Wellington, yes, and in Christchurch, but they definitely don't use it in Auckland. (Interviewer: they do use it in Auckland.) Really? Well, they must have been influenced by Christchurch then. SELF-CONSCIOUS is definitely from Christchurch and Wellington.

\section{EP2 SPEECH}

Is that SPEECH? That's a strong TC sign, SPEECH, I feel, because it's related to being taught how to speak. I don't use the sign at all and I don't know if anyone still uses it. It's an old sign, SPEECH. It can mean 'speak' or 'speech', but it's a very old or TC sign. I haven't seen it being used for a long time.

Figure 6.11: Comments related to variation in NZSL folk definitions 
Comments on variation were nevertheless interesting not only as a confirmation of salient sociolinguistic variables in NZSL, but also because they revealed attitudes held towards certain varieties. Some participants expressed a preference for older variants and regretted the wider adoption of more recent variants, for example in the case of two variants for HEARING shown previously in figure 6.2. Unfavourable remarks about 'TC' signs or 'English influenced' signs were frequent, but were paired with a recognition that these signs were in common use and were sometimes used by the participants themselves. Whereas a 'TC' sign such as ASK-2 (see figure 6.12) was particularly singled out for criticism when compared to the more 'culturally appropriate' ASK-1, no such comments were made for the similar pair DECREASE and LESS (see figure 6.2), and LESS was not recognised as a fingerspelling-derived (i.e. Englishinfluenced) form, possibly because the 'L' handshape of this sign is used in one-handed fingerspelling instead of in the two-handed alphabet in use in New Zealand.

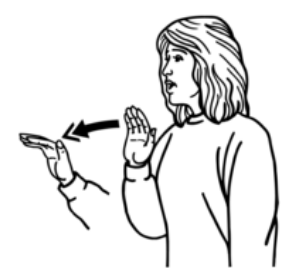

ASK-1

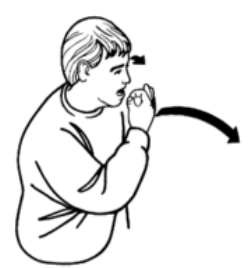

ASK-2

Figure 6.12: Two signs ASK in NZSL

\subsubsection{Grammatical characteristics}

The NZSL folk definitions not only integrated information about meaning and situational and social use, but also about grammar. Participants expressly drew attention to inflected forms of the target signs as part of their explanations. Table 6.6 shows examples of inflections for person, number and aspect that were commonly demonstrated. Video clips of these examples can be viewed by clicking on the hyperlinks or alternatively in Appendix $\mathrm{C}_{4}$.

The prevalence of inflectional morphology in the NZSL folk definitions may have a number of causes. Firstly, it may be that such inflections are in fact an integral part of a sign's meaning. For example, when shown the stimulus video of the sign BEEN-TO / HAVE-EXPERIENCE-OF, all participants queried which sign the video was showing and 'corrected' the production of the sign to include a more emphatic movement and the mouth pattern 'touch'. This corresponds with the observation by McKee and McKee (2013) that sign language models involved in filming sign entries for the ODNZSL found it difficult to produce uninflected forms of verbs and adjectives to serve as 'citation forms'. This indicated that "while these features [of 
inflectional morphology] are, in theory, optional, in natural usage they are closely fused with the lexicon." (p. 522)

\begin{tabular}{|c|c|c|c|c|}
\hline Target sign & Person & Number & Temporal aspect & $\begin{array}{l}\text { Manner and } \\
\text { degree }\end{array}$ \\
\hline DEAF & & & & $\begin{array}{l}\text { emphatic (very } \\
\text { much Deaf) }\end{array}$ \\
\hline \multirow[t]{2}{*}{ ASK/REMIND/TELL } & $\begin{array}{l}\text { I ask you } \\
\text { someone asks me }\end{array}$ & $\begin{array}{l}\text { plural (get } \\
\text { everyone's } \\
\text { attention) }\end{array}$ & & \\
\hline & I ask you to ask her & & & \\
\hline \multirow[t]{2}{*}{$\begin{array}{l}\text { TALK-IN-SIGN- } \\
\text { LANGUAGE }\end{array}$} & & $\begin{array}{l}\text { dual (one-to- } \\
\text { one) }\end{array}$ & ongoing & casual \\
\hline & & plural (group) & & a \\
\hline \multirow[t]{2}{*}{ DISCUSS } & & $\begin{array}{l}\text { dual (one-to- } \\
\text { one) }\end{array}$ & ongoing & serious, intense \\
\hline & & plural (group) & & \\
\hline $\begin{array}{l}\text { BEEN-TO / HAVE- } \\
\text { EXPERIENCE-OF }\end{array}$ & & $\begin{array}{l}\text { plural (been to } \\
\text { many places) }\end{array}$ & completed & $\begin{array}{l}\text { emphatic } \\
\text { (finally / with } \\
\text { satisfaction) }\end{array}$ \\
\hline \multirow[t]{2}{*}{ DECREASE } & & & $\begin{array}{l}\text { quick reduction } \\
\text { (LOSE WEIGHT) }\end{array}$ & \\
\hline & & & $\begin{array}{l}\text { dwindling over } \\
\text { time }\end{array}$ & \\
\hline
\end{tabular}

Table 6.6: Examples of inflected signs in NZSL folk definitions

Different inflections of a sign are sometimes associated with distinct senses. The general meaning DECREASE, when produced with a fast movement close to the body is used to express losing weight or dieting. When used with a gradual movement involving repeated rotation of the wrists, this signifies something dwindling or being decimated. While the fact that several inflected forms were produced by participants in response to the same stimulus video indicates that these forms are closely related, it is not always clear whether one of the senses is more central than the others. Even when inflections can be traced back to an uninflected form, for hearing language learners it may be difficult to retrieve the specific sense of the inflected form from its 'root'. For this reason, McKee and McKee (2013) argued that "frequent and semantically stable uses of productive verb morphology" should be included as separate dictionary entries. 
Secondly, inflected forms in the folk definitions point to the most typical forms in which these signs are used in normal discourse. For example, the sign BEEN-TO / HAVE-EXPERIENCE-OF is frequently used together with a completion marker (the sign FINISH / THEN-http:// nzsl.vuw.ac.nz/signs/3653) and also typically occurs in a plural form to indicate that there is a list of intended destinations or experiences that are being 'ticked off'.

Thirdly, participants' demonstration of different inflections were an indication of the grammatical category of the target sign. For example, the sign ASK/REMIND/TELL, belongs to an 'agreement verb' type that can be inflected for person and number through alteration of the movement of the sign to and from different locations in the signing space; and TALK-IN-SIGNLANGUAGE and DISCUSS can be inflected both for number and for temporal aspect.

\subsubsection{Cultural connotations}

A final aspect of folk definitions that was noted in the literature (e.g. Stock, 1992; Atkins \& Rundell, 2008) is that they provide evidence of cultural connotations that are part of native speakers' linguistic knowledge. The elicitation materials for the current study included several signs associated with Deaf cultural and communication practices and identity labels, and as a result many stereotypical connotations were evident in the NZSL folk definitions.

For some signs, the connotational meaning was more central than the denotational meaning. For example, many folk definitions of $\mathrm{CP}_{5}$ HEARING only mentioned the ability to hear sound as an afterthought. Instead, the defining characteristic of HEARING people was that they are not Deaf, and therefore do not use sign language. Several participants placed the sign in the context of introducing someone to a Deaf person. Identifying someone as HEARING in that context would implicitly make it understood that the Deaf person should be patient in communicating with them, sign slowly and assume that the person is not aware of Deaf cultural behaviours and norms.

Stock (1992) argued that in some cases, stereotypes persist even though the circumstances that gave rise to the stereotype have changed. As an example, she showed that the concept of a 'mother' as a married woman staying at home to look after the children is still evoked by the word 'mother', and that additional descriptions are necessary when the stereotype is contradicted by current cultural realities_-giving rise to expressions such as 'working mother' or 'single mother'. In the same way, Deaf participants in the current study acknowledged that some hearing people were competent sign language users and could even be considered to be part of the Deaf community, but these people were mostly identified with other labels such as 
INTERPRETER or CODA ('Child Of Deaf Adults'-i.e. a hearing child whose parents are Deaf and who has usually had exposure to sign language from birth).

As mentioned before, sign DC1 TALK-IN-SIGN-LANGUAGE was frequently produced with a plural inflection and accompanied by the attribute FOR-AGES. These attributes show that participants associated chatting in sign language primarily with large face-to-face gatherings of Deaf people. Taking part in such gatherings, mostly in Deaf clubs, has traditionally been a core element of Deaf community membership (Ladd, 2003). While current cultural practices may be changing due to the closure of many Deaf clubs and the rise of video and internet technology making it less necessary to pass on information face-to-face, stereotypical connotations in the folk definitions show that traditional cultural behaviours such as 'long goodbyes' are still valued highly.

When TALK-IN-SIGN-LANGUAGE was used in a particular inflected form, it carried a connotation of fluency in sign language. This connotation was not brought up in the folk definitions for the target sign itself. Instead, TALK-IN-SIGN-LANGUAGE was used by several participants to describe fluency in sign language as a criterion for being FULLY DEAF. This usage is interesting since it shows evidence of language change. The sign TALK-IN-SIGN-LANGUAGE was originally borrowed from ASL, in which language it is restricted to the grammatical dual form (i.e. it is used in the context of the signer having a conversation in sign language with one other person), and does not have the connotation of fluency (David McKee, personal communication, $7^{\text {th }}$ October 2016).

The previously mentioned comments that CC1 ASK/REMIND/TELL was a 'nicer' sign than the English-influenced ASK showed another cultural connotation, related to this sign's origin as a physical tap on someone's shoulder to get their attention. Since this behaviour reflects an awareness of the need to establish eye contact before commencing a conversation, it was seen as a uniquely 'Deaf' behaviour that was positively evaluated. This positive evaluation was then also applied to the sign when used in its current extended senses.

A final example of cultural connotations was found in definitions for sign EP1 SPEECH. Although only a few participants still recognised or used this sign, for those few participants the sign carried somewhat negative connotations. One participant associated SPEECH with SPEECH-TRAINING, i.e. a deaf person being taught how to articulate words. Other participants associated SPEECH with a Deaf person who would pride themselves in being able to speak 'like a hearing person'. Such connotations reflect the fact that the use of spoken language was 
historically imposed on Deaf people through the oral method (see section 2.1.2 - The status of sign languages).

\subsubsection{The form of NZSL folk definitions}

Having examined the content of NZSL folk definitions, I will now briefly report on selected features of their form. The aim of describing these features is not a detailed analysis of folk definitions as a genre, although such an analysis would be a useful addition to the still relatively scant description of genres in the sign linguistics literature. For the purpose of the current study, I will focus on a few aspects of the form of NZSL folk definitions that require consideration from the point of view of hearing language learners as the end-users of dictionary definitions.

\subsubsection{Lexical signals}

As was discussed in the literature review of this chapter, Flowerdew (1992) studied the form of spoken definitions in lectures to identify syntactic and lexical 'signals' that marked parts of the discourse as definitions. The elicited NZSL folk definitions were not embedded in a wider (teaching) context and therefore the beginning of a 'definition' was not usually marked or signalled in any specific way. In Flowerdew's data the words 'call / called' and 'means' were the most common lexical signals; however, the NZSL folk definitions only occasionally included the sign MEAN (http://nzsl.vuw.ac.nz/signs/1638) as an introduction to a definition. This sign was more frequently used to clarify the intended sense of a target sign (i.e. 'do you mean $x$ or y?'). The sign CAN (http://nzsl.vuw.ac.nz/signs/4501) was commonly used to signal that the offered definition only described one of several possible senses or situations of use. A number of other signs functioned as lexical signals of particular definition types. These signs and the corresponding semantic category that they occurred with are shown in table 6.7.

\subsubsection{Syntactic and grammatical structure}

As was described in section 2.2.3.1 - Simultaneity, one of the most striking characteristics of the syntax and grammar of sign languages is the use of simultaneous constructions.

Two kinds of manual simultaneity (where each hand contributes different information) were used to structure the NZSL folk definitions. Firstly, a common way of drawing attention to the sign being defined was to hold the sign in its location with one hand while the other hand pointed to it or signed further information about it. Examples are shown in figure 6.13. 


\section{Sign}

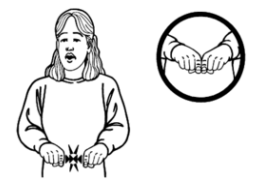

SAME / LIKE

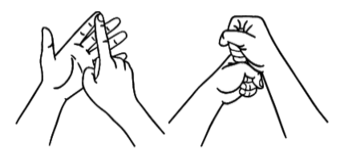

1

FOR-EXAMPLE

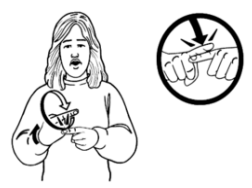

FOR-EXAMPLE
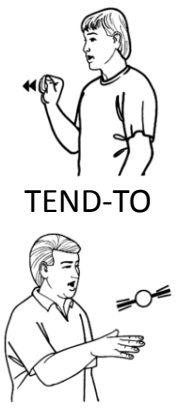

SIMILAR

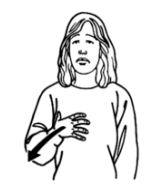

MORE

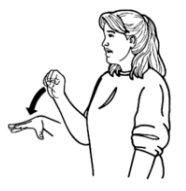

THAN

\section{Semantic category}

synonymy

exemplification

comparison

exemplification

exemplification

exemplification

attribution

Comparison

Comparison

Comparison

Table 6.7: Signs used to signal folk definitions in the NZSL data

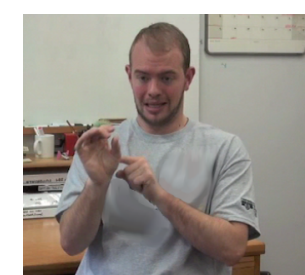

EC1 ONE-HANDED-FINGERSPELLING

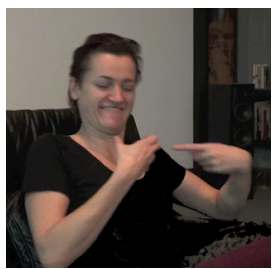

BC2 UNINTELLIGIBLE-VOICE

Figure 6.13: Simultaneous production of the target sign and referential pointing 
Secondly, participants frequently used 'list buoys' (Liddell, 2003; see figure 6.14 for examples of list buoys in NZSL). In list buoys, the non-dominant hand is held with one or more fingers extended. The dominant hand then points at or holds the extended fingers in turn and signs further information. List buoys are commonly used to structure not only enumerated lists of individual items, but also larger stretches of discourse, allowing the signer to return to previously mentioned referents. In the NZSL folk definitions, list buoys were used to distinguish between several senses or examples of use of target signs.
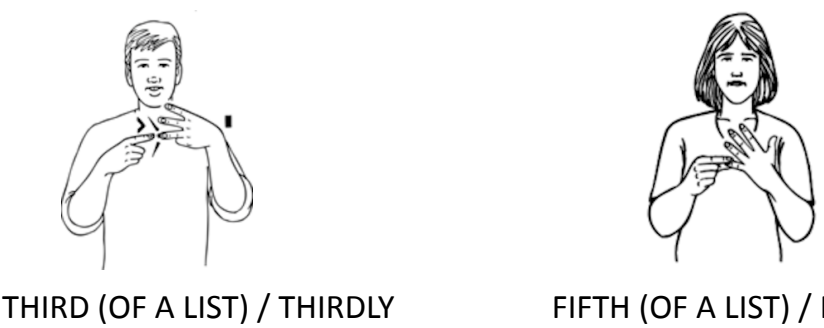

Figure 6.14: List buoys in NZSL

A further type of simultaneous construction consists of information being conveyed by various body parts at the same time as the manual signs. In the NZSL folk definitions, this kind of simultaneity was found in what has been called 'constructed dialogue' (Metzger, 1995), where the signer indicates through their eye gaze, body leans and other non-manual features that the utterance being signed should be attributed to a particular referent. Constructed dialogue allows a signer to change between the view points of several people, or to report what the signer themselves said or experienced at a different time or in a different place.

In the earlier figure 6.9, quotation marks in the translations indicate those parts of the folk definition for which constructed dialogue was used. These passages suggest that participants used constructed dialogue to demonstrate usage of the target sign in an imagined typical conversation.

Finally, the NZSL folk definitions included simultaneously produced manual and oral elements. Particular mouth gestures accompanied the inflected forms of target signs discussed in section 6.8.2.3 - Grammatical characteristics. Mouth gestures were also used by participants to identify different possible senses of target signs: for example, showing that sign $\mathrm{AC}_{4}$ KEEP-INSIDE-ONESELF when produced with lips pressed together refers to negative feelings such as grief or hurt, whereas the same sign produced with pursed lips can be used to refer to innate abilities, values or part of someone's identity. 


\subsubsection{Mouthing and fingerspelling}

As was predicted in section 6.4 - Signed definitions and explanations, all participants made use of strategies that showed code-mixing of NZSL and English to explain the meaning of particular signs. All four aspects of the non-native lexicon discussed in section 2.2.5 - The non-native lexicon were present in the data, i.e.: fingerspelling, mouthing, loan translations, and borrowings from other sign languages. Mouthing was used the most frequently, followed by fingerspelling. It was also noted in this previous section on the non-native lexicon that mouthing in particular is a pervasive element, with around $73 \%$ of sign tokens in NZSL discourse being accompanied by mouthing (McKee, 2007). It is therefore not surprising that folk definitions contain such non-native elements. In this section I will briefly examine the functions that mouthing and fingerspelling may fulfil in defining.

There were only three target signs that were not accompanied by mouthing in the folk definitions at any time. These target signs were: AP1 PUT-ASIDE; BC2 UNINTELLIGIBLE-VOICE; and EC4 I-WAS-WRONG / YOU GOT ME. These three signs were accompanied by mouth gestures (see section 2.2.5.2 - Mouth gestures and mouthing). Since the same mouth gestures were produced by all participants and on all occasions that the target sign was used, they can be considered to be a bound or conventional part of the sign. This is also reflected in the fact that the citation videos in the ODNZSL show the relevant mouth gestures. For these signs, mouthing is not available as a strategy for linking the meaning of the target sign to an English word. Other strategies such as synonymy and exemplification were therefore used to define these signs.

Three further target signs were produced with consistent mouthings:

- $\quad \mathrm{BP}_{5}$ DEAF-'full deaf';

- DC3 BEEN-TO / HAVE-EXPERIENCE-OF-'touch';

- $\mathrm{CP}_{5}$ HEARING—'hearing'.

These mouthings frequently represented abbreviated forms and did not always match the word class, morphology or semantics of the English equivalent (for example, 'full' instead of 'fully', or 'touch' when physical touch is not included in the figurative meaning of the sign). According to Schermer (2001), such mouthings are similar to mouth gestures in that they no longer represent complete English words and may be a conventional, stable part of the sign form. However, the usual mouthings were at times overridden when participants wanted to specify or emphasise a particular English translation. For example, $\mathrm{BP}_{5} \mathrm{DEAF}$ was also given 
the mouth patterns 'totally deaf' and 'profoundly deaf.' The replacement mouthings represented complete English morphology (e.g. 'totally' instead of 'total').

Other signs were produced without mouthing or with mouth gestures in parts of the folk definitions, but were produced with an English mouthing as a defining strategy. For example, sign AC4 KEEP-INSIDE-ONESELF was produced with the mouthing 'inside', in addition to being produced with different mouth gestures.

In common with the main functions of mouthing outlined in section 2.2.5.2 - Mouth gestures and mouthing, in my data mouthing was frequently used to disambiguate between different meanings of homophonous or polysemous signs. When presented with a citation video without a mouth pattern, participants would specify or ask which sense was intended by repeating the target sign with different mouthings: for example:

- $\quad \mathrm{AC}_{5}$ PlAIN/CONFIDENTIAL, PRIVATE-'sad', 'plain', 'private', 'secret'

- CC1 ASK/REMIND/TELL—'tell', 'ask', 'remind', 'attention', 'later'

- CC5 DEVELOP/GROW-'grow', 'develop', 'spring', 'volcano', 'plant'

- DC1 TALK-IN-SIGN-LANGUAGE- 'chat', 'talk'

- DP5 DECREASE- 'decrease', 'reduce', 'less', 'lose weight', 'small'

- EP2 SPEECH—'moan', 'complain', 'comment', 'speech', 'hearing'

A related function of mouthing-specifying the referent of generic polycomponential signs in a particular context (e.g. Schermer, 2001; McKee, 2007)—was also found in the data. Sign BC 5 SMALL-ROUND-FOOD-PLURAL was produced with mouth patterns such as 'grape', 'raisin', 'bean' and 'strawberry', specifying the particular foods that could be referenced by this sign.

Finally, the use of the mouthing 'small' as a complement to sign BC5 SMALL-ROUND-FOODPLURAL can be seen as a strategy to emphasise an intrinsic attribute of this depicting sign.

This brief overview of observed mouthing patterns in the data shows that the strategy of providing an equivalent in a different language was frequently employed in the signed folk definitions, in common with findings for spoken explanations and folk definitions (e.g. Chaudron, 1982; Nation, 2001). However, the role of mouthing (as a simultaneous bimodal expression) goes further than simply providing an English equivalent. In many instances participants commented that they could not tell which meaning was intended without a 
mouth pattern, or that a particular sign did not make sense at all without an accompanying mouthing. Such comments confirm, as McKee (2007) noted, that "(m)outhing is an integral feature of NZSL. English word forms and manual signs apparently co-exist closely in the lexicon of NZSL signers, and are systematically combined in a bi-modal meaning package." (p. 12).

Fingerspelling is used less frequently than mouthing in NZSL. Fewer instances of fingerspelling were therefore found in the data. However, with the exception of one target sign ( $\mathrm{CP}_{5}$ HEARING), the folk definitions for all target signs contained fingerspelled forms. Most frequently fingerspelled forms represented English equivalents of the target sign, but on a few occasions attributes or exemplifications of the sign were fingerspelled.

The functions and occurrence of fingerspelling in the folk definition data were very similar to those of mouthing and the two were frequently used in sequence as part of disambiguation or specification of the target sign. Typically, fingerspelling followed mouthing, suggesting that the fingerspelled form is perceived as a more exact specification of an English synonym than the mouthed form.

When used to differentiate between different senses of polysemous signs, or to specify a sense of generic signs, some fingerspelled forms represented common senses: for example, s-a-d and p-l-a-i-n for target sign $\mathrm{AC}_{5}$, and p-e-a, b-e-a-n, g-r-a-p-e and s-e-e-d for target sign $\mathrm{BC}_{5}$. Less common, more specific senses of the sign were also fingerspelled, e.g. f-l-u-o-r (flourish), GROW s-p-u-r-t, and b-l-o-s-s-o-m-e-d for target sign CC 5 DEVELOP / GROW; i-n-n-a-t-e for sign AC4 KEEP-INSIDE-ONESELF; or n-o-t-i-f-y for sign CC1 ASK/REMIND/TELL.

A single manual letter form was only found once in the data ( $v-v$ for 'volcano') even though this category is much more frequent in NZSL than entire fingerspelled words (Pivac Alexander, 2008). Apart from a few lexicalised fingerspelled acronyms and abbreviations ( bb (baked beans), A-T-M, A-S-L, and A-G-M), all other occurrences were full fingerspelled words or phrases. Fingerspelling in the folk definitions was used across a broad range of word classes, including nouns (b-e-a-n, p-l-a-n-t), verbs (c-h-a-t, a-s-k, c-o-m-m-e-n-t), adjectives (i-n-n-a-t-e, -p-la-i-n) and entire phrases (f-u-l-l-y-d-e-a-f). This is in contrast with the use of fingerspelling in NZSL in general, which according to Pivac Alexander was predominantly restricted to nouns (proper and common nouns) and included no instances of fingerspelled verbs. 
Taken together, these patterns suggest that the majority of fingerspelled forms were part of a deliberate codeswitching or 'translanguaging' (Garcia \& Wei, 2014) defining strategy rather than reflecting typical usage of fingerspelling in NZSL discourse.

All participants used fingerspelling; however, sociolinguistic variation in NZSL fingerspelling patterns (Pivac Alexander, 2008) was also evident in the folk definitions data. One participant in particular included English morphology in the fingerspelled form (e.g. b-e-a-n-s and b-lo-s-s-o-m-e-d), whereas other participants omitted English morphemes (e.g. g-r-a-p-e for the inherently plural sign $\mathrm{BC}_{5}$ ). The same participant also fingerspelled English bound morphemes in combination with a sign: for example, d-e-REDUCE for 'decrease' and u-n-EXPECT for 'unexpected'.

\subsection{Summary of findings}

The findings from the folk definition elicitation study can be summarised as follows:

\section{Semantic categories}

- Semantic categories used to explain the meaning of signs in NZSL were similar to those found in folk definitions in other (spoken) languages.

- The most frequently used semantic categories were synonymy and exemplification, followed by comparison and attribution.

- Synonymy was most frequently used to describe idiomatic signs and to identify different senses of polysemous signs.

- Exemplification was used to explain verbs and adjectival signs, and to compare signs with similar senses but different semantic and colligational restrictions, by providing examples of abstracted hypernyms as well as typical instances of use drawn from personal experience.

- Attributive, spatial, functional, operational, and class inclusion categories were less frequently used since many of these relationships are inherent in signs that are visually motivated, especially polycomponential / depicting signs.

- Antonymy was used to describe adjectival signs. 


\section{Other characteristics}

- Information about register and situations of use was provided as an inseparable part of the meaning of signs

- Sociolinguistic factors such as age and region as well as external influences from English were salient to participants, but did not always reflect actual variation in NZSL.

- The signs being defined were frequently shown in a range of inflected forms.

- Many of the folk definitions contained stereotypical connotations that provide an insight into cultural values and behaviours of Deaf NZSL users.

The form of NZSL folk definitions

- Signs such as LIKE, SIMILAR and MORE functioned as lexical signals of specific semantic definition types.

- Simultaneous constructions were common, including manual, manual/non-manual and manual/oral simultaneity.

- Manual simultaneity included holding target signs with one hand and referring or commenting on the sign with the other hand, as well as the use of list buoys to distinguish between different senses or usage examples.

- Constructed dialogue was used to exemplify how a target sign could be used in conversation.

- Mouth gestures were used to distinguish between alternate senses of target signs.

- Mouthing and fingerspelling were frequently used to disambiguate or specify senses. While some mouthings were a conventional part of the sign form, other mouthings and all fingerspelled forms were used as deliberate strategies to provide English equivalents.

\subsection{Limitations}

As with previous studies in this thesis, the relatively small number of participants is the main limitation of the present study. Nevertheless, the study generated close to 10 hours of discussion around the semantics and contextual usage of the 15 target signs. As was mentioned previously, transcription and annotation of signed texts is extremely timeintensive. I was therefore unable to transcribe all of the discussions in full. Instead I opted to code the relevant semantic and grammatical categories in NVIVO and transcribe only selected extracts. This approach may have limited the emergence of other frequent usage 
patterns from the data, since any phenomena that were not coded were not transcribed and therefore not searchable.

Since the target signs used to elicit the NZSL folk definitions were selected on the basis of a previous test with hearing NZSL learners, the signs were of restricted categories and included metaphorical and idiomatic expressions. This contrasts with studies such as Casagrande and Hale (1967) that primarily elicited folk definitions for concrete objects and actions. The preference for certain semantic categories in the NZSL folk definitions can therefore not be generalised to definitions of the NZSL lexicon as a whole.

As mentioned before, my own presence as a hearing person may have influenced the register Deaf participants used in their explanations, especially in the three interviews where no other Deaf people were present.

Finally, the Deaf person who conducted the interviews in Wellington asked participants to describe who would use the target sign, where it was used, and whether there were any other sign variants in use. These prompts may have led participants to concentrate on variational factors rather than on explaining the meaning of target signs. However, comments on sign variation were also common in the Auckland data. The fact that Deaf NZSL users, including the Deaf interviewer, saw variation as salient confirms that the context, including situational and sociolinguistic variation, is a closely connected aspect of explaining how a sign is used.

\subsection{Conclusion}

In this chapter I have made a case for eliciting folk definitions as a method to gather data about the meaning and usage of signs as well as a model for signed dictionary definitions. The first research question for the study reported on in this chapter was whether common semantic, grammatical and discourse features could be identified in signed folk definitions. The results of an analysis of the elicited folk definitions show that there were indeed a number of typical features that were common across NZSL users and across groups of target signs. As a data collection method, elicited folk definitions were shown to provide rich semantic data from a relatively small group of participants. While it is acknowledged that large-scale corpus analysis provides the most objective foundation for lexicography, it is also evident that sign language corpora are, and are likely to remain, much more limited in size and scope than written or even spoken language corpora. Given these circumstances, elicited folk definitions are a practical supplement to analysing general conversational data. What 
distinguishes this proposed method from reliance on native sign language user introspection is that folk definitions can be elicited from multiple informants and that the informants themselves do not have to decide whether a particular usage is typical or not. Instead, later analysis reveals cognitive prototypes that can then be called upon in compiling the dictionary definition.

The second research question asked which of the features of signed folk definitions could be drawn on in creating planned dictionary definitions. The first set of features to consider is the semantic categories that were most frequently employed in the folk definitions: synonymy, exemplification and comparison.

With regard to synonymy, Atkins and Rundell (2008), amongst others, have cautioned that providing a synonym or series of synonyms by itself is not sufficient to define a word. The main disadvantage of defining by synonymy is of course the scarcity of true equivalence. As can be seen from the data in figure 6.2, many signs offered as 'synonyms' at times only corresponded with one sense of a polysemous sign. Other 'synonyms' were themselves polysemous and only corresponded to the target sign in one sense. For example, defining a polysemous sign such as PLAIN/CONFIDENTIAL/PRIVATE/SAD with another polysemous sign PLAIN/ SMOOTH/FLAT ... would increase ambiguity rather than reduce it.

Other synonyms belonged to particular registers or variants that might unintentionally evoke particular attitudes or connotations when used by learners. As was found in the user study of the ODNZSL (see section 4.6.4.2 - Making sense of search results), learners were confused by the presence of multiple variants in the search results. Further guidance in the form of additional information on variation and register would be a valuable addition to sign language dictionaries, but is unfortunately difficult to achieve due to the sociolinguistic factors noted elsewhere in this thesis. The use of sign variants as synonyms in defining may therefore be problematic.

Exemplification as a defining strategy provided contextual information on the situations in which a sign can be used, as well as showing colligational restrictions. This information is crucial for learners, especially for language production. However, Stock (1988) maintained that dictionaries could convey this information (as well as further grammatical information) better through examples of use rather than as part of a definition. The ODNZSL is one of the few sign language dictionaries that already contains corpus-informed signed examples. Currently these examples are coordinated with the main English glosses in the entry as these 
glosses are the only representation of a sign's sense. If signed definitions on video were introduced to the entry, the relationship between this video and the example videos would need to be considered. It may be that some broad exemplification categories (e.g. FEELINGS for KEEP-INSIDE-ONESELF, or NUMBER for DECREASE) could be presented as part of the definition while more specific examples are presented separately. Exemplification in the folk definition data could also be used to confirm whether the most typical examples were chosen in cases where there are few tokens in the corpus. For example, the ODNZSL entry for UNINTELLIGIBLE-VOICE describes a Deaf woman's singing voice, while the folk definitions for this sign suggested that it is more typically used to describe men and may be linked specifically to the use of one's voice while signing.

Comparing and contrasting the semantic and grammatical features of two or more signs is a potentially useful approach to dictionary definitions in some cases. As was seen in the folk definition data, signs related to hearing status were defined in relation to each other through grading and antonymy. The comparison of two signs DECREASE revealed a reasonably complex network of more and less typical co-occurring signs. In both these cases, cross-references to related signs would be beneficial for dictionary users. It may be possible to create more detailed definitions that treat groups of related signs rather than defining each sign in isolation.

Although there were few signed folk definitions using attribution, operational, functional, or spatial relationships, the inherent reflection of these relationship types in some classes of signs may not be transparent to learners. Making these properties explicit in dictionary definitions may be helpful.

The next aspect of folk definitions discussed in this chapter was the embedding of contextual information on sociolinguistic variation and situational use. As previously mentioned, it would be inadvisable to incorporate comments on variation from the folk definitions into the dictionary without further verification through other means. However, when it comes to conveying such information (once verified) to learners, signed dictionary definitions could be modelled on the integrated structure of the folk definitions. In other words, in addition to providing register information separately through tags such as 'informal' (currently used in the ODNZSL), dictionary definitions could incorporate a typical context of use, including clues to the field, tenor, and mode of the situation. 
Grammatical information was also frequently highlighted in the folk definitions, in particular inflections for person, number, temporal aspect and manner and degree. In the ODNZSL, such inflections are listed in the sign entry through a set of labels below the word class(es) (see figure 6.15). However, as was indicated in section 6.3 - Folk definitions in dictionaries, learners may benefit from seeing such grammatical patterns included in contextualised form in the dictionary definition rather than as a separate label. Current example sentences in the ODNZSL mostly focus on demonstrating non-inflected forms where these exist. It would be possible to include further example sentences with inflected forms as an alternative to including this information in the definition.
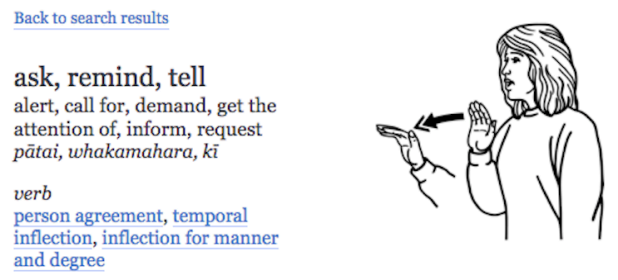

Figure 6.15: Grammatical inflection labels in the ODNZSL

I have argued that one of the main contributions of using signed folk definitions as a template for dictionary definitions is that they offer semantic and usage information about signs from a native point of view, including culture-specific connotations. Although Stock (1992) argued strongly that cultural connotations are crucial to learners' understanding of texts, she also conceded that dictionaries are limited in their capacity to convey such connotations. Atkins and Rundell (2008) similarly cautioned that it may be difficult to determine precisely which connotations are essential for learners' understanding. Given these reservations, the treatment of cultural connotations in dictionary definitions should be approached on a case-by-case basis.

In acknowledgement of Sinclair's (1987 b) and Hanks' (1987) view that the form in which folk definitions are expressed is inseparable from the meaning of the word being defined, this chapter identified a number of lexical and grammatical features of NZSL folk definitions. Lexical signals that indicate the relationship between the sign being defined and its description will be a suitable component of dictionary definitions. Grammatical patterns such as list buoys and constructed dialogue are typical of a large part of signed discourse. Such patterns would therefore be good candidates for including in a template for signed dictionary definitions. However, simultaneous constructions and the use of space are also features in which the grammar of sign languages is most distinct from spoken languages. It 
can therefore not be assumed that learners of a sign language, even at intermediate level, will find these constructions easy to understand.

Lastly, the frequent presence of (originally) non-native elements such as fingerspelling and especially mouthing in the signed folk definitions adds complexity to decisions around the form signed dictionary definitions should take. On the one hand, mouthing is typical of language use in the New Zealand Deaf community and in some cases, may be an integral part of the sign. Mouthing may also provide a connection between the learners' lexicon in their first language and the sign they are trying to learn. On the other hand, if mouthing and fingerspelling are seen as a code-mixing phenomenon, then they may not be suitable models for learners' sign language production. In addition, when explaining the meaning of a sign, the underlying problems of non-equivalence between English words and signs would not be resolved by relying on representations of English words, whether these representations are in the form of a written gloss or as a fingerspelled or mouthed word. In light of ongoing debate around the status of mouthing and how it should be reflected in sign language dictionaries, no particular recommendations will be made here with regard to the inclusion of mouthing in signed dictionary definitions. However, I would argue that fingerspelling should be avoided in the definitions since it does not add any more information than is already provided in the written English glosses and is likely to be more difficult for learners to understand.

In conclusion, this study of elicited folk definitions has not only provided a detailed description of the semantics and usage of a small selection of NZSL signs, but has also outlined a tentative model for the construction of dictionary definitions, drawing on aspects of the form of folk definitions.

In applying this model, three further factors will need to be considered. Firstly, it will be important to verify that signed definitions as presented in the dictionary are still perceived as acceptable and typical by other native sign language users. Secondly, care will be required in the editing and re-filming of signed dictionary definitions in order to preserve the helpful features of the folk definitions while avoiding less desirable characteristics of informal, spontaneous production such as circularity, repetition and hesitations. Finally, the extent to which signed dictionary definitions meet the user needs of the main target group of hearing (intermediate) language learners will need to be evaluated. The next and final chapter will address these three factors. 


\section{Chapter 7: From folk definition to dictionary definition}

\section{Introduction}

In this chapter I turn to the practical application of the findings of the previous three studies to the development of twelve pilot dictionary definitions. The chapter follows on directly from the description of common semantic and grammatical features of folk definitions described in the previous chapter, where I concluded that folk definitions are a fruitful source of semantic data and contain typical grammatical and usage patterns that may be useful for language learners. I will describe the steps taken in moving from the use of folk definitions as data, to their use as templates for dictionary definitions.

I will first explore the results of focus group discussions with the Deaf participants who contributed the folk definitions. While dictionary definitions should ultimately meet the needs of hearing language learners, it is important that the definitions remain examples of natural and typical sign language use. The focus groups therefore aimed to evaluate which aspects of the form of signed definitions (both informal folk definitions and more formal definitions in overseas sign language resources) were considered to be valuable and appropriate by Deaf sign language users.

Next, I will outline the process of editing and filming the pilot dictionary definitions. I will describe how I selected typical semantic and grammatical features of the folk definitions and then worked together with Deaf colleagues to create definitions that aimed to preserve these typical features of folk definitions while minimising less helpful characteristics inherent in informal explanations, such as lengthiness, circularity, hesitations, and complex grammatical structures.

Finally, I will examine the effectiveness of the resulting pilot dictionary definitions by reporting on a repeat of the error identification and correction test that was introduced in Chapter 5 - Evaluating the user need for definitions with a new group of hearing intermediate NZSL learners. The unmodified test items were used to compare the performance of the two student groups to ensure that they were matched. Mean scores on the twelve modified test items before and after the addition of the pilot definitions were then compared. The implications of the test results will be discussed together with comments on the definitions made by the student participants in a short post-test review discussion. 


\subsection{Deaf perspectives on definition formats}

It was important to gather the opinions of Deaf NZSL users on the suitability of a more informal explanation format and on other factors that should be considered in the move from folk definitions to signed dictionary definitions. As well as wanting to ensure that a Deaf perspective was prioritised throughout the creation of new dictionary information, Deaf NZSL users' feedback at this stage would be essential in confirming that my analysis of the folk definition content and format corresponded with Deaf people's own perceptions of the data.

While it would have been valuable to survey a large part of the Deaf community and to allow for open participation in this process, there were both practical and methodological obstacles to such an approach. Firstly, the folk definitions under evaluation were inseparable from the people who provided them and responses by the wider Deaf community would most likely be influenced by subjective perceptions of these people rather than the content and format of the definitions themselves. Secondly, the disadvantages of surveys and questionnaires that were noted in section 4.4 - Methodology of user research are also applicable here. Thirdly, although an online questionnaire or survey could present both the definitions under evaluation and the questions as signed videos, multiple-choice questions would provide limited information, whereas open questions would require the capability of filming responses in NZSL. Submitted video responses would not be anonymous. Finally, given the findings of the user study in Chapter 4 - A user study of an online sign language dictionary that Deaf people are infrequent users of the ODNZSL, the response rate to a general survey might be low.

Instead of a larger-scale survey, I therefore decided on a focus group format with the participants of the folk definition elicitation study. The recent experience of having to explain the meaning of signs would have highlighted some of the difficulties of creating definitions for these participants; and as NZSL teachers, they would have an insight into learners' needs and how signed information is best presented to learners. Reviewing the folk definitions with the original participant group would also allow the participants to compare their own folk definitions with those of others in a non-threatening confidential context. 


\subsubsection{Method}

\subsubsection{Materials}

Two separate sets of materials were discussed and evaluated in the focus groups. The first activity and discussion were aimed at gathering feedback on the folk definitions collected as part of the study described in the previous chapter (Chapter 6 - Folk definitions). To introduce and guide the discussion of the folk definitions, I developed an activity using four of the 15 target signs. The selected signs were:

- $\mathrm{AC}_{4}$ KEEP-INSIDE-ONESELF;

- $\mathrm{BP}_{5}$ DEAF;

- CC5 DEVELOP/GROW;

- DC3 BEEN-TO / HAVE-EXPERIENCE-OF.

I selected video extracts of four different participants' folk definitions of each of these four target signs. Overall, the 16 selected video clips contained at least one sample from every participant in the folk definition study. I developed a rating sheet that included a table with still images of the target signs, together with numbered still images of the four folk definition extracts. Written English information on the rating sheet was deliberately kept brief. Participants were asked to view the video clips and then circle the number of the folk definition that they considered to be the overall best explanation of that target sign. Next, the rating sheet asked participants to write down the number of the folk definition(s) that they rated as being:

- Easiest to understand;

- Most natural NZSL;

- Most full information;

- Most appropriate for Deaf people;

- Most appropriate for hearing NZSL students.

During the second part of the focus groups, participants were asked about the format dictionary definitions should take. Since there were no existing signed definitions in current general sign language dictionaries that could be shown as examples to facilitate the discussion around this subject, I selected two alternative resources that illustrated a variety of explanation formats: 
1. http://www.ssc.education.ed.ac.uk/BSL/list.html: The British Sign Language Glossaries of Curriculum Terms (Scottish Sensory Centre, n.d.) is a set of technical dictionaries / glossaries for the fields of mathematics, science and astronomy, predominantly aimed at Deaf high school students (and their teachers and sign language interpreters). Many of the signs in this glossaries include definitions, signed by Deaf professionals in the relevant fields who were also the consultation group for the development of the glossaries. The definitions are varied in style and length; some include the use of additional visual material (e.g. a graph, or an image of the defined object as a full screen background to the signer) or physical props (e.g. a scale model of an asteroid). An example is shown in figure 7.1.

2. http://www.signs2go.eu: The Signs 2 Go online British Sign Language course for Deaf learners was previously discussed in section 6.4 - Signed definitions and explanations. The explanations in Signs 2 Go are different from the format found in technical sign language dictionaries, in that they provide contextual explanations of vocabulary used in larger dialogues. The sample explanation chosen for the focus groups was an explanation of how to use Signs 2 Go, in which the signing figure was projected close to the object they were referring to (see figure 7.2). This presentation style means that the explanations have a direct context for references to other objects: for example, the signer can simply point at the position of a button on the screen rather than explaining that the user should click on a button with a particular name. Since this kind of interactivity may be particularly helpful for dictionary users, I wanted to include this presentation style in the focus group evaluations.

Due to the close historical relationships between BSL and NZSL and a considerable overlap in the lexicon (McKee and Kennedy, 200o, estimated that the languages have $79 \%$ of identical or related signs in the core lexicon), the content of both resources was comprehensible to the Deaf NZSL using participants.

An intended rating activity of these different explanation formats along scales including length, formality, intelligibility, visual clarity and personal preference proved to be too cumbersome during the Auckland focus group. The rating sheet was therefore amended for the Wellington focus group to include a list of the example explanations shown without an associated paper activity. 
Finally, some general questions around the format and content of signed definitions were posed. A copy of the focus group rating and discussion sheet is included in Appendix D1.

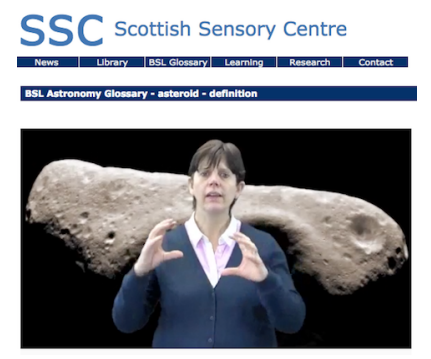

Figure 7.1: Definition of asteroid in the BSL Glossaries of Curriculum Terms (http:// www.ssc.education.ed.ac.uk/BSL/astronomy/asteroidd.html)

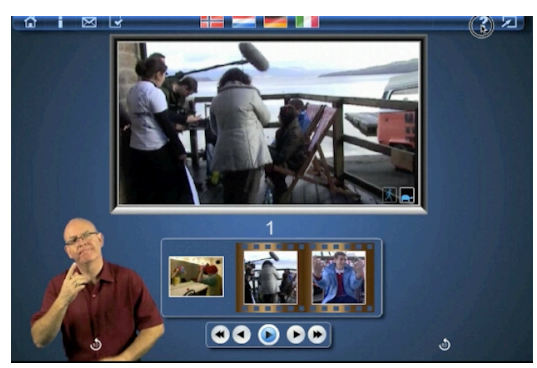

Figure 7.2: Contextualised presentation of signed explanation in Signs 2 Go (http:// www.signs2go.eu/)

\subsubsection{Participants}

All 13 participants in the folk definitions elicitation study (see 6.7.2 - Participants) were invited back to take part in the focus groups. Nine of the original participants were available: five in Auckland and four in Wellington.

\subsubsection{Procedure}

Two focus groups were held, in Wellington and Auckland. Both focus groups were held in a university classroom. Deaf facilitators were recruited to manage the discussion in NZSL. In Auckland, the facilitator was one of the original participants. In Wellington, a separate facilitator was recruited. The focus group discussions were recorded using two video cameras. Before the start of the focus group, the facilitator clarified ground rules regarding confidentiality, turn taking and keeping comments impersonal and focused on general characteristics of the definitions.

Next, I introduced the purpose of my research into signed definitions. An example of an (online) English dictionary entry and an ODNZSL entry were shown to compare what 
information was provided in each entry. I drew attention to the lack of definitions in the ODNZSL and reiterated the purpose of including definitions by Deaf NZSL users. I stressed that the focus of the group discussion would mainly be on the format such definitions should take, and their broad content, rather than on individual sign meaning. After this introduction, I withdrew myself from the discussion circle although I remained present to operate the video cameras.

The remainder of the focus group was structured around the activities outlined on the rating sheet. Participants were first shown extracts from the folk definitions and were asked to rate these individually on the sheet. A facilitated discussion of these ratings followed. After a break, the sample definitions from the two BSL sign language resources were shown and discussed. Finally, the facilitator guided a discussion on the format and content of signed definitions, using the questions provided on the rating sheet. The length of the focus groups was around 2 hours.

\subsubsection{Results}

\subsubsection{Evaluation of NZSL folk definitions}

There was little agreement between participants' ratings of individual folk definitions. Not only were the ratings between participants different, but individual participants rated definitions differently against different criteria. As a result, nearly all definitions received at least one vote for each of the criteria, i.e. best explanation; easiest to understand; most natural NZSL; most full information; most appropriate for Deaf dictionary users; and most appropriate for hearing NZSL learners. The differences in number of votes received were especially small in the 'best explanation' category. This suggests that a diverse range of criteria was being used to judge what made a folk definition a good explanation.

Nevertheless, some patterns emerged from examining the relationships between the criteria. Focusing only on those definitions that received the most votes for each criterion, figure $7 \cdot 3$ shows how often several criteria coincided for these top ranked definitions. For example, this figure shows that there were no instances when a definition was chosen to be both the best explanation and the most natural NZSL use. From this figure it can be deduced that participants were mostly guided by what they thought would be the most appropriate definition for hearing NZSL learners, and that they also judged definitions that provided the fullest information as being the best explanations. 

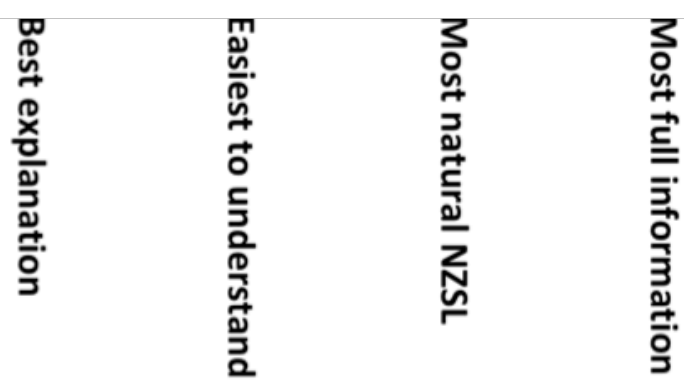

\section{Best explanation \\ Easiest to understand \\ Most natural NZSL \\ Most full information \\ Most appropriate for Deaf people \\ Most appropriate for hearing learners}
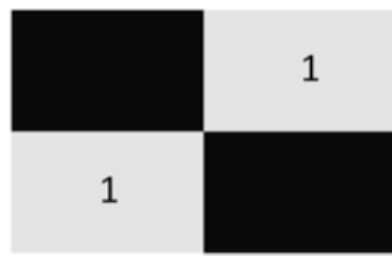

1

2

13

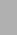

\begin{tabular}{|c|c|c|c|c|c|}
\hline 2 & 0 & 1 & & 1 & 3 \\
\hline 1 & 0 & 2 & 1 & & 1 \\
\hline 3 & 2 & 3 & 3 & 1 & \\
\hline
\end{tabular}

Figure 7.3: Correspondences between rating criteria for the top rated folk definitions

Although natural NZSL use did not emerge as a main criterion by which the folk definitions were judged, it was associated with definitions that were easy to understand and appropriate for both Deaf people and hearing learners.

Participants also identified features that they liked and disliked in the folk definitions. They indicated that the best explanations were clear and concise, were produced with a slow or moderate signing speed, and contained contextual examples and grammar information about the sign. Synonyms, comparisons between related signs, and showing signs in a number of inflected forms were all commented on positively.

Participants liked that the folk definitions highlighted Deaf perspectives by giving examples from real life contexts and that informal 'slang' usage examples were given alongside more formal senses. The overall casual style of the folk definitions was also seen as positive. Interestingly, in contrast with definitions appropriate to hearing learners requiring all of these features, the main criterion for rating definitions as appropriate for Deaf people was that the definition contained examples. 
The most natural NZSL use, while conducive to the clarity of the definition and appreciated for the use of non-manual features, was also connected with repetition and diverging from the main topic. The length of some of the folk definitions was the most commented on feature that made them unsuitable as dictionary definitions.

There were a number of contradictions in the judgments made about the folk definitions. For example, it may be difficult to reconcile the preference for definitions that provide the fullest information and plenty of examples with the requirement to be concise and to the point. Similarly, participants commented favourably on the relaxed, casual style of the folk definitions but rated some definitions negatively because of being too informal. Facial expression was also the subject of disagreement, with positive comments about animated signing with good use of non-manual features and affective facial expression, but also negative evaluations of 'too much' facial expression. Some participants liked definitions where the meaning of a sign was explicitly related to, or contrasted with, English words and constructions, whereas other participants judged some folk definitions as showing too much English influence.

\subsubsection{Evaluation of other definition formats}

Participants applied similar criteria to their evaluation of the signed definition videos from overseas resources as they did in rating the folk definitions. In both cases, the preference was for in-depth definitions that included examples. Definitions from the British Sign Language Glossaries of Curriculum Terms (Scottish Sensory Centre, n.d.) were mostly considered to be an appropriate length and level of detail for the technical subject matter and intended younger audience. For instance, participants liked the clear examples for the sign PROPERTIES (http://www.ssc.education.ed.ac.uk/BSL/chemistry/propertiesd.html\#start). However, the three examples given for this sign—breakability (iron vs. wood), pH level (acid vs. water) and flammability (water vs. petrol) _ added to the length of the definition and it was generally agreed that shorter definitions with one or two clear examples would be more suitable for general purpose sign language dictionaries primarily aimed at adult users.

As was previously mentioned in section 3.4.4 - General vs. restricted, newly coined signs for technical concepts often draw on iconic or metaphorical visual features. Definitions for these signs may then draw attention to the motivation linking the sign and the concept. 
Participants commented that such explanations would help hearing learners to remember the form of the sign and were in favour of including 'hints' or explanations of a sign's visual properties in the definitions.

In terms of the form of definitions in the BSL Glossaries and in Signs2Go, the relatively casual style of these definitions was found to be an appropriate model. Participants especially liked definitions where the signer addressed the camera directly as if they were having a conversation with the dictionary user. An example is the definition of ODD (http:// www.ssc.education.ed.ac.uk/BSL/maths/bslodddef.html\#start) in which the signer explains the concept by dividing an odd number of apples between 'me' (the signer) and 'you' (the viewer).

Differences in the structure of the definitions were not found to be problematic, although it was perceived as helpful to conclude a definition by repeating the sign that was being defined. Opinions differed as to whether the sign being defined should also be introduced at the beginning of the definition, since the sign form would most likely already be shown in a separate video and it would add to the length of the definition if it were included again. There were comments on the lack of facial expression and mouth patterns in some ODNZSL videos showing signs in 'citation form', and it was agreed that if the sign was being introduced in the definition, it should be accompanied by relevant facial expression or mouthing.

In terms of visual presentation of the definitions, it was agreed that the video should be presented in its own screen with minimal visual distractions, preferably in wide screen format with the signer's upper body in full view. The full screen background shown in figure 7.1 and the layout of the Signs2Go screen in figure 7.2 were both seen as too distracting. While graphical support was found to be helpful in some cases (for example, an image of a DNA strand accompanying the definition of the sign GENE in the BSL Glossaries), in other cases participants felt that illustrations might introduce ambiguity rather than clarify the concept. Some participants also mentioned that the use of illustrations would potentially detract from the authoritative image of a general purpose sign language dictionary.

The relationship between English text and signed videos in the dictionary entry was also discussed. English glosses of the sign were important, either as a caption or in their current form, but participants saw no benefit in including an English equivalent in fingerspelled form. Different views were expressed on the question whether the signed definition itself should be translated into English. Although an English translation was considered more 
accessible for learners, some participants thought that the translation would dominate and might lead to the signed definition being ignored.

With regard to relationships between signs (e.g. homonyms, synonyms, or signs in a specific semantic field), it was considered useful to include hyperlinks to other entries in the dictionary entry, but there was no support for the proposal of creating more detailed definitions covering groups of related signs.

The final part of the focus group discussion was around register, variation and grammar information. All participants agreed that this information was essential; however, it was not always thought necessary to convey this information as part of the definition. Links to a separate grammar section, or written labels or tags, were seen as an adequate solution. An exception was the treatment of inflections in dictionary entries. Participants were strongly in support of demonstrating inflections as part of the definitions. Some participants argued that 'adverbial' inflections such as intensifiers were too general to include in a definition, since these inflections could be applied to a large set of signs. These kind of inflections might be better treated in a separate grammar section.

Throughout the discussion, participants commented that there was a need for flexible dictionary interfaces so that information could be presented in a variety of ways to suit individual users: for example, toggling between viewing the definition video and additional information such as translations, supporting visual material, and examples.

\subsubsection{Summary}

Overall, positive responses from the focus groups to informal explanations with embedded contextual examples and grammar information, along with the recognition that the definitions showed authentic Deaf points of view, confirmed the proposition that folk definitions are a suitable model for signed dictionary definitions. Definitions should mainly be edited for length and signing speed, and avoid repetition. There was no support from the focus groups for the idea of combined definitions of groups of related signs.

Although focus group participants' comments indicated that current register, variation and word class labels were adequate and that there was not always a need to include such information in a signed definition, the user study in section 4.6.4.2 - Making sense of search results showed that the current labels are not always understood by (hearing) dictionary users. In light of arguments presented in the previous chapter that favoured contextualised 
embedding of such information in the definition, it would be appropriate to include such elements of the folk definitions in the creation of edited dictionary definitions.

A theme that has emerged from different parts of this thesis is that dictionary information may need to be presented in a variety of ways to suit different user groups. Feedback from the focus groups again endorsed flexible electronic interfaces, although it was stressed that any video materials (such as signed definitions) should be clearly presented in their own screen without too much other visual information.

\subsection{Developing dictionary definitions: editing and filming}

The outcomes of the focus group discussions contributed a series of guidelines to the proposed model of developing dictionary definitions based on characteristics of folk definitions. Next, this model had to be tested in practice. By creating a small pilot set of dictionary definitions, I would be able to assess the viability of the editing and filming process as well as evaluating whether the proposed definition format is effective in meeting the needs of language learners. In the following section I will describe in some detail how pilot dictionary definitions were developed for the target signs that formed the stimulus for the folk definition elicitation study (see table 6.1).

\subsubsection{The editing process}

The first step in creating the pilot dictionary definitions was to select the essential semantic content and aspects of the form of the folk definition data for each target sign. In the analysis of the folk definitions reported in the previous chapter (see 6.7.4 - Analysis), I had included any features that were used by more than one participant for the same target sign. In order to select the most typical patterns, during the editing stage a stricter benchmark was applied: a particular feature had to have been used by at least three participants before it was considered for inclusion in the final dictionary definition.

Inevitably, decisions had to be made about whether certain sign senses should be included and whether they should be grouped together or treated separately. I decided to include only those senses that were treated in the existing ODNZSL dictionary entry or entries that formed the basis of the error correction test in section 5.6.2 - Developing the test instrument. For example, target sign $\mathrm{C}_{5}$ was included in the error correction test on the basis of the senses 'develop' and 'grow” in dictionary entry http://nzsl.vuw.ac.nz/signs/3567. Any folk definitions of the sign used in these senses were therefore considered. However, the sense 
'volcano' (shown in dictionary entry http://nzsl.vuw.ac.nz/signs/5075 in a slightly different form), although it met the benchmark in terms of the number of participants who suggested this sense, was omitted at this point. References to the senses 'hearing' and 'complain, moan' in relation to sign EP2 SPEECH were also excluded. Similarly, senses that were not currently covered in the ODNZSL were omitted from the pilot dictionary definitions. This included folk definitions of $\mathrm{AC}_{4}$ KEEP-INSIDE-ONESELF with an alternative facial expression referring to 'innateness' or parts of a person's identity; responses to BC2 UNINTELLIGIBLE-VOICE that referred to deep laughter; and folk definitions showing an inflected form of DC1 TALK-IN-SIGN-LANGUAGE to indicate fluency in sign language.

During editing, sign DECREASE 2 (http://nzsl.vuw.ac.nz/signs/1294) was substituted for sign DECREASE 1 (http://nzsl.vuw.ac.nz/signs/249) in order to be able to include this definition in the error correction test, where a test item required looking up information in relation to DECREASE 2.

During the selection process, it became clear that for a small number of target signs, the folk definitions contained insufficient information relating to the sense(s) in the dictionary entry to form the basis of a dictionary definition. Three signs were therefore not taken forward to the filming stage. These three signs were: $\mathrm{AC}_{5}$ PLAIN / CONFIDENTIAL / PRIVATE; AP1 PUT-ASIDE; and EC1 ONE-HANDED-FINGERSPELLING.

For each of the remaining twelve target signs, a list of relevant aspects of semantic content and form was compiled, including typical examples of use, synonyms, inflection patterns, comments about register and other components of the folk definitions that had been identified as useful. I also took note of any signs used in the explanations that might not be in the vocabulary of intermediate learners. Where it was not possible to eliminate lower frequency signs from the definition altogether, I selected signs that are included in the ODNZSL.

\subsubsection{Sign model}

The selection of a Deaf person to model the signed definitions was informed by McKee and McKee's (2013) description of factors that guided the recruitment of sign models for the ODNZSL. These factors included authenticity of language use, metalinguistic awareness, familiarity with being filmed, and the ability to perform scripted or rehearsed language on cue. This last factor is particularly relevant to the filming of signed definitions. The presence of cameras and the awareness of a larger eventual audience may lead a speaker or signer to 
accommodate to perceived norms of how they 'should' communicate (Bell, 1991) and therefore the language modelled may differ greatly from the casual register found in the folk definitions. While accommodation effects cannot be avoided, especially when working with only partly scripted or rehearsed language, a sign model who is used to being in the public eye and addressing a mass audience would be better able to 'perform' natural looking language under these circumstances.

A further consideration in choosing a sign model was the planned use of the signed definitions in a test, where the definition videos would appear alongside existing example videos from the ODNZSL. For a fair assessment of the effectiveness of the definitions, It was important to minimise factors other than their content that would distinguish them from existing dictionary material. It was therefore decided to select a sign model who had previously been involved in modelling filmed material for the ODNZSL. To ensure some consistency of style in this small set of videos, all pilot definitions would be signed by the same person.

Although the sign model had not been able to take part in the focus groups, she had been a participant in the elicitation of folk definitions reported on in the previous chapter. Her involvement in the study ensured that the sign model had an understanding of the nature of folk definitions and the purpose of the current thesis.

\subsubsection{The filming process}

In addition to the sign model, another Deaf consultant was recruited to assist with filming. As was the case with filming the folk definitions, the presence of another Deaf person as an immediate audience was expected to influence the sign model's language choices (reducing the use of contact varieties more typically used with hearing addressees) and to encourage the use of a more casual register. The Deaf consultant could also provide feedback and prompts during filming.

The filming environment was set up to be consistent with the ODNZSL in terms of clothing, background, and framing.

General guidelines about the form of the definitions were discussed, including the lexical signals and grammatical structures discussed in section 6.8.3 - The form of NZSL folk definitions. It was agreed that 'list buoys' (see figure 6.14) would be used to indicate different senses of a sign. Since it was not clear from the focus group discussions whether the target 
sign should be introduced at the beginning of the definition, the decision was left to the sign model. After trying to sign alternate versions of one of the definitions, the sign model suggested that it felt more natural to begin definitions with the target sign. This pattern was therefore followed for all the definitions.

Next, the content of each target sign definition was discussed with the sign model and the Deaf consultant. The target sign itself was shown to the sign model in the form of a drawing and the citation form video from the ODNZSL entry, but the English equivalents were not shown. Written bullet points listing the features that should be included in the definition were used as prompts for discussion. The content was not scripted, however. After discussion and clarification, which occasionally involved viewing the relevant parts of the folk definitions as a direct model, the sign model practised signing the definition as a whole, trying different ordering of the information and different introductions and endings. The Deaf consultant and myself provided feedback. When the overall structure of the definition had been agreed, it was recorded on camera. The Deaf consultant provided signed prompts for the next part of the definition. This process of discussion, rehearsal and filming was repeated for each sign.

After the definitions had been filmed, they were reviewed and edited, primarily to ensure that the signing speed and the overall length of the definition were appropriate.

As was predicted in section 6.3 - Folk definitions in dictionaries, even when the definitions were rehearsed in advance, features of spontaneous production such as hesitations could not be avoided altogether. It was very difficult for the sign model to memorise not only content but also particular phrasing and sign constructions. An additional difficulty was that the sign model at times was asked to present definitions of signs that she herself did not use, or used in a different sense. Along with these cognitive demands on the sign model, an awareness of the eventual hearing audience as well as the presence of the camera also had an impact on the sign model's language production. Due to these factors, the sign model's production, although clear, was rather fast. The videos were therefore slowed down to $80-85 \%$ of their original speed.

A few definitions (including the definition for target sign $\mathrm{CP}_{5}$ HEARING) were found to be too long or to contain explanations that were not clearly expressed. In these cases, unclear phrases and superfluous examples were removed. 
The resulting twelve pilot dictionary definitions are presented in Appendix $\mathrm{D}_{3}$ or can be viewed via the links in table 7.1.

\begin{tabular}{|c|c|}
\hline Target sign & Link to definition video \\
\hline AC4 KEEP-INSIDE-ONESELF & https://youtu.be/nXPhJHfR-iQ \\
\hline BC2 UNINTELLIGIBLE-VOICE & https://youtu.be/o5b2yBm1RUE \\
\hline BC5 SMALL-ROUND-FOOD-PLURAL & https://youtu.be/IqBe_MENCbY \\
\hline BP5 DEAF & https://youtu.be/GCv2uYhUjJU \\
\hline CC1 ASK / REMIND / TELL & https://youtu.be/t6v7ZFfSt88 \\
\hline CC5 DEVELOP / GROW & https://youtu.be/kzlQCkl7GcA \\
\hline CP5 HEARING & https://youtu.be/x_VD5tVZB9w \\
\hline DC1 TALK-IN-SIGN-LANGUAGE & https://youtu.be/dSRPkaEgw0s \\
\hline DC3 BEEN-TO / HAVE-EXPERIENCE-OF & https://youtu.be/IPs5SduODgo \\
\hline DP5 DECREASE & https://youtu.be/I1iYBQLbkaQ \\
\hline EC4 I-WAS-WRONG / YOU-GOT-ME & https://youtu.be/WZyaRbVM3rs \\
\hline EP2 SPEECH & https://youtu.be/phw5i32NoSO \\
\hline
\end{tabular}

Table 7.1: Twelve pilot dictionary definition videos

\subsubsection{Evaluation of the pilot dictionary definitions}

To evaluate whether the editing and filming process described in this section succeeded in creating dictionary definitions with the desired characteristics of folk definitions, I will revisit the criteria that were set out at the beginning of the process.

The first of these criteria was that definitions should be concise and to the point. The length of the pilot dictionary definitions ranged from 16 seconds to 54 seconds, with an average of 34 seconds. This means most definition videos were nearly five times as long as a single example video (with an average length of 7 seconds). We should take into account that several definitions described more than one sense or usage, which would equate to more than one example. The length of the definitions can mostly be attributed to the inclusion of detailed register, grammatical and cultural information, reflecting the preference for full descriptions noted in the focus group discussions. However, unlike the folk definitions, the pilot dictionary definitions contain little or no repeated information or digressions. Examples and 
synonyms in the definitions are also generally restricted in number, although several brief examples are provided when these were thought to illustrate frequent usage in signed discourse. For example, the definition for $\mathrm{CP}_{5}$ HEARING includes three examples: HEARING WORLD; HEARING CHARACTERISTIC; and HEARING MIND (WAY-OF-THINKING).

The signing speed in the pilot definitions, as mentioned previously, was faster than was deemed suitable for the target group of hearing intermediate language learners. The edited speed (8o-85\%) was similar to the speed of signing in those folk definitions that were identified as slow and clear. However, feedback from hearing language learners should be sought to confirm that the edited speed was appropriate.

The clarity and comprehensibility of the pilot definitions can also not be assessed without feedback from the end users. As was described previously, efforts were made to restrict the use of low frequency signs and complex grammatical structures in the definitions. For example, although the folk definitions contained a large number of synonyms for sign $\mathrm{EC}_{4} \mathrm{I}$ WAS-WRONG / YOU-GOT-ME, the definition only included EMBARRASSED and OOPS (see figure 6.2): signs that were more likely to be familiar to intermediate learners. Attempts to restrict the vocabulary used in the dictionary definitions were not always successful. For example, the same definition for sign EC4 also contains the sign BEAT (in the context of one team beating another team during a competition). This particular sign variant is not included in the ODNZSL and may not be familiar to learners.

Semantic categories used in the folk definitions were mostly maintained in the pilot dictionary definitions as planned. For example, exemplification was used in the definition of $\mathrm{DP}_{5}$ DECREASE, and attribution was included for BC2 UNINTELLIGIBLE-VOICE ('rough') and DC1 TALK-IN-SIGN-LANGUAGE ('comfortable'). Although the dictionary definitions treated signs individually rather than as a group, comparisons with other relevant signs were maintained in some cases. For example, $\mathrm{CP}_{5}$ HEARING included a reference to hearing people being different from DEAF people; and DC1 TALK-IN-SIGN-LANGUAGE included a contrast with TALK-TO-EACH-OTHER (http://nzsl.vuw.ac.nz/signs/4301).

The focus groups had identified the demonstration of inflections and appropriate nonmanual features as important elements of the definitions. The pilot dictionary definitions include inflections for DC1 TALK-IN-SIGN-LANGUAGE and DC3 BEEN-TO / HAVE-EXPERIENCE-OF. Target signs are shown with relevant mouth gestures and mouthings: for example, puffed cheeks with DP 5 DECREASE and the mouth pattern 'touch' with sign DC 3 BEEN-TO / HAVE-EXPERIENCE-OF. 
The use of different mouthings to indicate different senses of a sign is explicitly referred to in the definitions for BC5 SMALL-ROUND-FOOD-PLURAL and CC5 DEVELOP / GROW.

The pilot definitions for the two target signs $\mathrm{BC}_{5}$ and $\mathrm{CC}_{5}$ also draw attention to the visual properties of these depicting signs, by outlining their shape and emphasising their movement. In the case of $\mathrm{CC}_{5}$, a link is also made between the sense of physical plant growth and the metaphorical use of this sign to refer to inner growth and development.

Connotations and information about usage are also included selectively in the pilot definitions. For example, the connotation of DC 1 TALK-IN-SIGN-LANGUAGE with FOR-AGES is shown (see section 6.8.2.4 - Cultural connotations), and the definition for BC2 UNINTELLIGIBLEVOICE mentions that it is considered rude for a hearing person to comment on a Deaf person's voice quality using this sign. The pilot definition for sign CC 1 ASK/REMIND/TELL was originally filmed with an explanation that this sign is preferred by many Deaf people over the Englishinfluenced sign ASK (http://nzsl.vuw.ac.nz/signs/3009). However, this information and a demonstration of different inflections for this sign were omitted from the final version due to the overall length and concerns about the comprehensibility of this part of the definition.

As was indicated previously, the aim was to produce definitions in an informal style, using lexical signals and grammatical structures found in the folk definitions. The completed pilot definitions include some of these features, including the planned use of list buoys to distinguish between different senses, and the signalling of examples with the sign FOREXAMPLE (http://nzsl.vuw.ac.nz/signs/5530). The sign model addresses the dictionary user directly at times (e.g. stating that sign $\mathrm{BC}_{5}$ is 'something you eat') and maintains eye contact with the camera. Many definitions include first person examples.

In other respects, however, the pilot dictionary definitions diverge from the typical patterns found in the folk definitions. The sign SAME / LIKE (http://nzsl.vuw.ac.nz/signs/2479) was used very frequently in the folk definitions, but was rarely used as a lexical signal by the sign model. When this sign did occur in the pilot definitions, it was accompanied by the mouth pattern 'also' and was not used to indicate synonymy, exemplification or comparison, but instead pointed to additional information in general. Another feature-the simultaneous manual construction in which the signer held the target sign with one hand while pointing to it with the other hand (see section 6.8.3.2 - Syntactic and grammatical structure)—was used much more frequently in the pilot definitions than in the folk definitions. During rehearsals, the sign model began most definitions with the phrase “[TARGET SIGN] MEAN WHAT", a 'rhetorical 
question' construction that is roughly equivalent to the classical signalling phrase "[this word] means..., observed by Flowerdew (1992) as an introduction to (formal) spoken definitions. The signed phrase was also found in many definitions in the BSL Glossaries of Curriculum Terms (Scottish Sensory Centre, n.d.). In the folk definitions, however, this phrase did not occur at all, and the sign MEAN was only rarely used. During filming, different introduction phrases more closely resembling the folk definitions were tried out, but the sign MEAN and the rhetorical question construction still occur frequently in the final pilot definitions. The more frequent use of rhetorical question constructions has also been observed in other contexts when Deaf people have been asked to present information on video to a non-present audience (Rachel McKee, personal communication, $15^{\text {th }}$ November 2016). It may be, then, that this usage was a natural result of the decontextualised production of the definitions to camera, which required that the definitions be introduced in a more formal way.

The pilot definitions also show other effects of decontextualisation, including references to 'a person' who is not the signer or the viewer. Two different ways were used to indicate a neutral person: firstly, the sign model outlined her own upper body with both hands; and secondly, the sign model combined two different 'classifier' forms (see section 2.2.4.1 - Depicting signs or polycomponential signs) that are more commonly used independently to refer to a 'person': the lexicalised form recorded in the ODNZSL (http://nzsl.vuw.ac.nz/signs/4446), and the upright index finger. Figure 7.4 shows both of these constructions. Neither of these constructions were typical in the folk definitions, and the sign model herself only produced the second of these constructions once during the folk definitions.
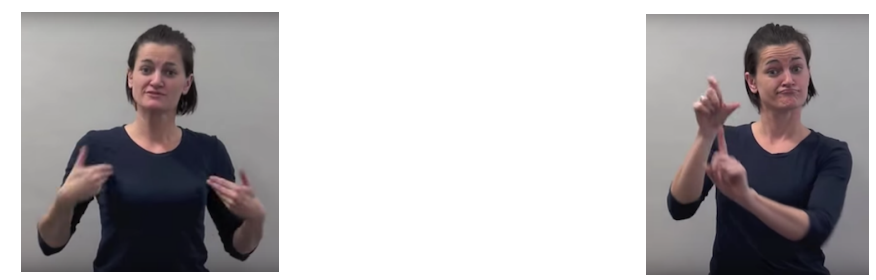

Figure 7.4: References to 'person' in the pilot dictionary definitions

Finally, the predicted accommodation towards a hearing audience (see section 7.2.2 - Sign model) was evident in the more frequent use of English mouthing and the more deliberate, clear and somewhat emphatic production of signs throughout the pilot definitions. 


\subsubsection{Summary}

This section has outlined the practical steps taken to create twelve pilot dictionary definitions. An evaluation of these definitions showed that they retained many features of folk definitions. The editing and filming process succeeded in eliminating a number of characteristics of spontaneous sign language production such as repetition and hesitations while still allowing for spontaneous production by not completely scripting the definitions.

The re-filming of the definitions under decontextualised conditions led to differences in style between the folk definitions and the dictionary definitions, including influences from English and some idiosyncratic productions. These factors detract from the typicality of language patterns that was one of the original motivations for using folk definitions as a model. The introduction of atypical patterns may be an issue for language learners trying to model their own language production on the usage observed in the definitions. If certain constructions are used more frequently in definitions than in sign language discourse in general, then this may lead to difficulties. For example, it has been noted anecdotally that constructions using a 'rhetorical question' tend to be overused by hearing learners. Although it may not be possible to avoid the presence of the camera and an awareness of the (hearing learner) target audience from influencing the sign language production of the sign model, further editing, rehearsing and re-filming might mitigate against particular stylistic features becoming problematic.

The fact that three target signs had to be excluded due to lack of data suggests that the proposed method of analysing folk definitions as the basis for the dictionary definitions may not always be feasible. A further drawback of this method is the amount of time involved in reviewing folk definitions, selecting relevant aspects and rehearsing definitions for re-filming. In spite of these limitations, I have demonstrated that it is possible to take folk definitions as a starting point for developing dictionary definitions.

The evaluation of the pilot dictionary definitions in this section has also shown that the definitions met the requirements that emerged from the Deaf focus groups. The final step in evaluating the suitability of this dictionary definition format is to ask whether it meets the needs of the eventual dictionary users. In the next section, I will address this question. 


\subsection{Testing the effectiveness of dictionary definitions}

In the final part of this chapter I will report on a tentative measure of the effectiveness of the pilot dictionary definitions in meeting the productive and receptive needs of hearing, intermediate sign language learners. I used the test instrument developed in section 5.6 Method for this purpose, with some modifications that will be described below. For ease of reference, I will refer to the original test as 'Test 1 ' and the current test, which included the definitions, as 'Test 2'.

\subsubsection{Method}

\subsubsection{Materials}

The online error identification and correction Test 1, described in section 5.6.2 - Developing the test instrument, was adapted to include the newly created definition videos for the twelve test items. These test items will be referred to as the 'modified' test items. The remainder of the test items were 'unmodified' in that no new information was added. However, to reduce the possible impact of a difference in 'time on task' between modified and unmodified test items, participants were shown the existing example sentence video from the ODNZSL entry for test items that did not have a definition. Since participants were already directed to make full use of the ODNZSL information for each test item, the example sentence video did not add further information, but ensured that the question format was consistent.

An example of the revised test format for Test 2 is shown in figure 7.5, and the revised instructions are shown in figure 7.6. The complete modified test instrument is shown in Appendix D4.

Due to the elimination of three definitions in the editing stage, modified test items were not distributed evenly between test blocks. Block A only contained one modified test item; Block E contained two modified items; all other blocks contained three modified items: two in the comprehension part and one in the production part. The fact that some participants may have answered fewer modified test items than others was taken into consideration in the analysis. 
c) Production

Watch this video which shows a version of the target sentence in NZSL:

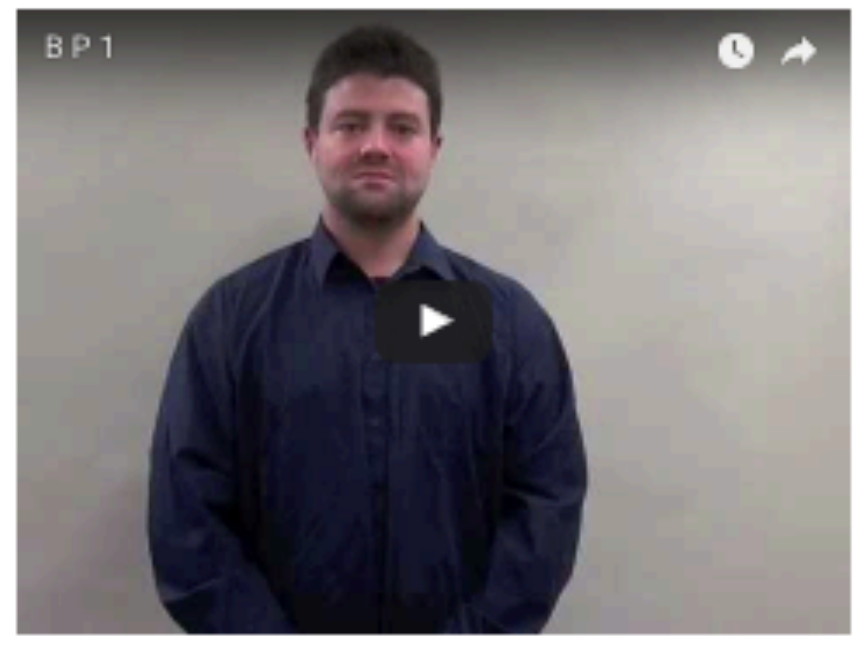

The target word or phrase is shown in this still image. Click on the image to view information about this sign in the Online NZSL Dictionary.

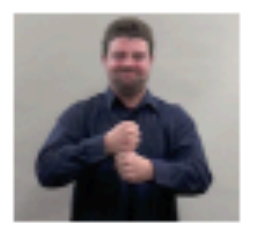

Now watch this video about the target sign:

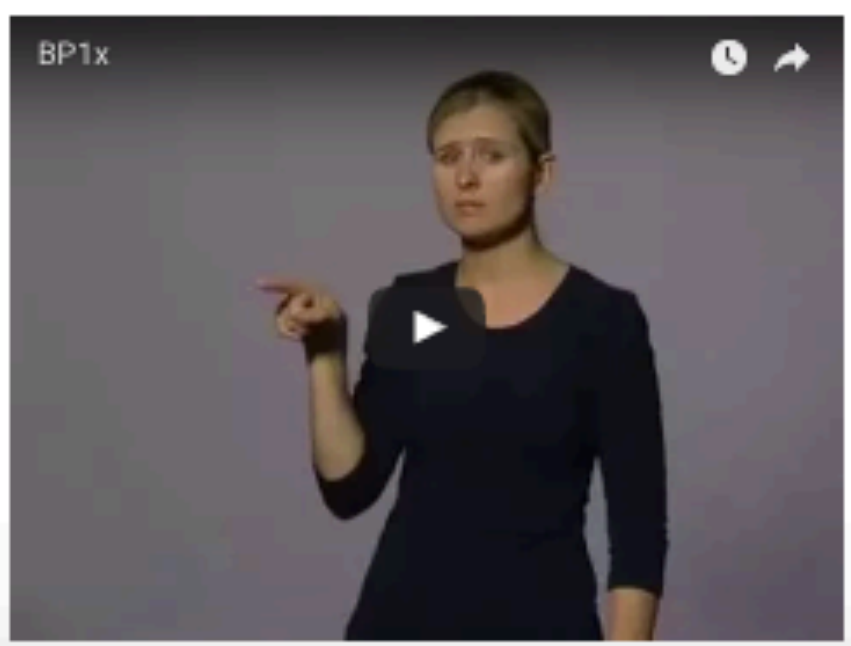

Figure 7.5: Revised test item format (production section) including an example or definition video 


\section{PART 1: COMPREHENSION}

In this part of the exercise, you will first be shown a video of a sentence in NZSL.

Next, you will be asked to look at information about a target sign or sign phrase in this sentence:

1) Look up information about this sign in the Online NZSL Dictionary. You can also look up other signs in the sentence if you are not sure what they mean.

2) Watch a video about the target sign. This could be one or more example sentences showing how the sign can be used in a sentence, or an explanation of the meaning of the sign.

Then you will see an English translation of the first NZSL sentence.

Your task is to decide whether the translation is correct or not.

If you think the sentence has an error, you will be asked to correct it. You do not have to type the entire sentence again; just type a replacement for the error.

\section{PART 2: PRODUCTION}

In this part of the exercise, you will first be shown an English sentence. Imagine that you are preparing to translate this sentence into NZSL.

1) You will be asked to look up a target word or phrase in the Online NZSL Dictionary. To do this, you will click on an icon. This will open up the home page of the dictionary. Type your search terms in the box labelled 'search by word (English/Maori)', then press 'search'.

2) Then you will see a video with the target sentence translated into NZSL.

You will be asked to once again look at information about a target sign from this sentence in the Online NZSL Dictionary. You will also watch a video about this target sign. This could be one or more example sentences showing how the sign can be used in a sentence, or an explanation of the meaning of the sign.

Your task is to decide whether the translation is correct or not.

Figure 7.6: Revised test instructions for comprehension and production parts

\subsubsection{Participants}

Although it was not possible to match subjects exactly with those who took Test 1 , participants were recruited from the same two intermediate classes and the test was taken at the same time of year, ensuring that the number of hours of formal NZSL learning participants had undertaken prior to the test was consistent. A total of 18 participants took part in Test 2.

\subsubsection{Procedure}

As previously, Test 2 was scheduled as part of a regular language lab. The procedure was identical to Test 1 (see section 5.6.4 - Procedure). 
Due to technical difficulties, two of the participants had to restart the test on a different computer and two other tests were not closed off until later. It was therefore not possible to take an accurate measurement of the average time participants took to complete Test 2.

\subsubsection{Results and discussion}

\subsubsection{Quantitative analysis}

First, individual participants' scores were compared between Test 1 and Test 2 to establish whether the two participant groups were matched. Four partially completed submissions in Test 2 were excluded since it was not possible to identify which of these partial test responses were provided by the same participants re-starting the test on a different computer.

The mean participant score for Test 2 was $70 \%$ (range $25-95 \%$, SD $=15.8$ ). This compared with a mean participant score of $68.6 \%$ in Test 1 . A paired t-test revealed no significant difference: $\mathrm{t}(35)=0.26, \mathrm{p}=0.8$ (2-tailed).

Overall test scores per item were also compared. As per Test 1, all test items were given a score by calculating the mean number of correct responses for that item. The overall mean score per item in Test 2 was 69\% (range o-100, SD 26.9). A paired t-test showed that there was no significant difference between the overall mean scores of Test $1(\mathrm{M}=71 \%)$ and Test $2(\mathrm{M}=$ $69 \%): \mathrm{t}(98)=0.45, \mathrm{p}=0.65$ (2-tailed).

A further paired t-test was carried out to compare the mean scores of only the 38 unmodified test items. Test 1 achieved a higher mean score $(M=74.21 \%)$ than Test $2(M=69.84 \%)$; however, again the difference was not significant: $\mathrm{t}(74)=0.75, \mathrm{p}=0.46$ (2-tailed).

Since no significant differences were found between the two participant groups on any of these three measures, the groups were considered sufficiently matched. Therefore, the scores of the modified test items in Test 1 (before a definition was added) and Test 2 (with the inclusion of a definition) can be compared to measure whether the definitions had any effect.

A paired t-test comparing the mean scores of the 12 modified test items in Test $1(\mathrm{M}=61.2 \%)$ and Test $2(\mathrm{M}=65.5 \%)$ yielded $\mathrm{t}(22)=-0.44, \mathrm{p}=0.66$ (two-tailed). Although in contrast with unmodified items, Test 2 scores were higher for modified items, the overall effect of adding definitions was therefore not significant.

Finally, to account for the possibility that Test 2 results were influenced by the additional time participants spent watching a video (whether a repeated example sentence in the case of 
unmodified test items, or a definition video in the case of modified test items), a two-way factorial analysis of variance (ANOVA) was carried out. Results are presented in table 7.2.

\begin{tabular}{llcccc}
\hline & Sum of squares & df & $\begin{array}{l}\text { Mean } \\
\text { square }\end{array}$ & F & P \\
\hline Source & 128.37 & 1 & 128.37 & 0.2 & 0.6557 \\
$\begin{array}{l}\text { Modification of test item } \\
\text { (unmodified or modified) }\end{array}$ & 1376.13 & 1 & 1376.13 & 2.16 & 0.1449 \\
Interaction & 349.06 & 1 & 349.06 & 0.55 & 0.4601 \\
Error & 61093.85 & 96 & 636.39 & & \\
Total & 62947.41 & 99 & & & \\
\hline
\end{tabular}

Table 7.2: Two-way factorial Analysis of Variance of modified and unmodified test items in Test 1 and Test 2

No significant effects or interaction effects were found.

Since it was found in Test 1 that participants sometimes identified errors but were not able to correct them appropriately, the paired t-tests and two-factorial ANOVA were repeated using the adjusted scores for error correction.

In Test 1, the mean score per item dropped when error correction was included in the analysis. In Test 2 , the opposite effect was noted: the mean score per item for error correction $(\mathrm{M}=70 \%)$ was higher than for error identification $(\mathrm{M}=69 \%)$. This can be explained by the fact that some participants identified the wrong part of the sentence as incorrect, but in their correction showed that they were able to understand or produce the correct utterance. Only for the 12 modified test items in Test $\mathbf{2}$ did the mean score drop when taking error correction into account: $M=66 \%$ for error identification, $M=62 \%$ for error correction.

None of the repeated tests comparing Test 1 and Test 2 using the adjusted error correction scores showed any significant differences: statistical details will therefore not be reported on here.

The conclusion of this statistical analysis is that the pilot dictionary definitions did not have a significant effect on hearing language learners' comprehension and production of NZSL signs. Although this is a disappointing result, it may partly be due to the method by which Test 1 and Test 2 scores were compared. The small sample sizes of the tests mean that a statistical analysis based on mean scores could be influenced considerably by one or two very 
low or very high scoring test items. For example, the mean score across all 12 modified items in Test 2 was reduced due to the very low scores on one test item (for $\mathrm{BP}_{5} \mathrm{DEAF}$ ).

Table 7.3 shows that if the modified test items are compared one by one, the error correction scores of 9 out of the 12 items increased after adding definitions, i.e. $75 \%$ of the scores were higher in Test 2 than they were in Test 1 . When the same comparison is made between unmodified items, 16 out of 38 scores were higher in Test 2 , i.e. only $42 \%$. It may be the case that there was a 'flattening' effect since lower scoring items were chosen as target items for definitions, and therefore there was more room to increase scores than in the unmodified items. However, the stronger increase in scores across modified test items still suggests that definitions had some effect. Furthermore, whereas in Test 1, 6 items scored less than 50\% (i.e. less than half of the participants could correct the error), in Test 2 this was reduced to 4 items.

\begin{tabular}{|c|c|c|c|}
\hline Test item & $\begin{array}{l}\text { Mean error } \\
\text { correction score (\%) } \\
\text { Test } 1\end{array}$ & $\begin{array}{l}\text { Mean error correction } \\
\text { score (\%) } \\
\text { Test } 2 \text { (with definitions) }\end{array}$ & Comparison \\
\hline AC4 KEEP-INSIDE-ONESELF & 44.4 & 77.8 & $\uparrow$ \\
\hline BC2 UNINTELLIGIBLE-VOICE & 50.0 & 75.0 & $\uparrow$ \\
\hline BC5 SMALL-ROUND-FOOD-PLURAL & 37.5 & 42.9 & $\uparrow$ \\
\hline BP5 DEAF & 37.5 & 14.3 & $\downarrow$ \\
\hline CC1 ASK / REMIND / TELL & 25.0 & 66.7 & $\uparrow$ \\
\hline CC5 DEVELOP / GROW & 75.0 & 83.3 & $\uparrow$ \\
\hline CP5 HEARING & 37.5 & 75.0 & $\uparrow$ \\
\hline DC1 TALK-IN-SIGN-LANGUAGE & 75.0 & 88.9 & $\uparrow$ \\
\hline DC3 BEEN-TO / HAVE-EXPERIENCE-OF & 62.5 & 66.7 & $\uparrow$ \\
\hline DP5 DECREASE & 14.3 & 42.9 & $\uparrow$ \\
\hline EC4 I-WAS-WRONG / YOU-GOT-ME & 77.8 & 62.5 & $\downarrow$ \\
\hline EP2 SPEECH & 66.7 & 42.9 & $\downarrow$ \\
\hline
\end{tabular}

Table 7.3: $\quad$ Comparison of Test 1 and Test 2 error correction scores for modified test items 


\subsubsection{Further analysis}

In light of this one-by-one comparison of test items, it might be appropriate to revise the initial outcome that definitions had no effect at all. However, since no significant effects could be statistically proven, additional qualitative measures are once again required before a final conclusion can be drawn.

As mentioned previously in section 5.3 - Examples, Nesi's (1996) consideration of three factors in her study on the effectiveness of examples can be adapted to the evaluation of the test results in the current study. To summarise these three possible factors: firstly, the information in the pilot dictionary definitions may not have been located or used by test participants; secondly, the test method may have been flawed; and thirdly, the definitions may not have provided the right information.

With regard to the first factor, it is certainly possible that some test participants did not view the dictionary definition videos. Although the instructions in the test explicitly directed participants to view all information, it was noted during Test 1 that the short test completion times of some participants made it unlikely that they had in fact consulted the dictionary during the task. Test 2 completion times were not recorded accurately and it is therefore not possible to confirm whether all participants viewed the definitions. A further possibility is that participants may have assumed that all videos were a repeat of information that they had already viewed during the dictionary consultation part of the test questions. This may have led them to ignore the definition videos.

With respect to test validity, the limitations of the original test instrument that were mentioned in section 5.9 - Limitations apply equally to the modified test. These limitations include the previously mentioned small sample size of participants with a wide range of NZSL proficiency. There were also problems with some test items not measuring the issue they were constructed to measure. For example, it was noted that some responses to test item AC4 KEEPINSIDE-ONESELF attributed the act of 'feeling hurt, but keeping it to oneself' to the wrong person due to the lack of an overt subject in the test sentence. The addition of a definition for this test item cannot be expected to have an impact on this wider grammatical issue, and unsurprisingly, the same kind of incorrect responses were found in Test 2.

To assess whether low test scores were due to the content of the definitions, I reviewed whether the definitions provided the information required to resolve the error in the test items. Of the 12 definitions, 9 explicitly made reference to semantic or grammatical 
information that was directly relevant to the error in the test. A further 2 definitions (for signs CC1 ASK / REMIND / TELL and DC1 TALK-IN-SIGN-LANGUAGE) demonstrated correct usage and semantic content but may not in themselves have been sufficient to prevent the error in the test. The final definition, for $\mathrm{BP}_{5} \mathrm{DEAF}$, is worth examining in more detail since this test item was not only the lowest scoring item in Test 2, but also saw a decreased score compared to Test 1 . The error in this test item was a collocation issue: it relied on learners recognising that this sign phrase cannot be used in an adjectival position in phrases such as 'Deaf sports'. The definition did not provide any information that would have helped learners with this grammatical issue, since it focused on semantic content. Moreover, the explanation of what it means to be 'fully Deaf' focused on participation in Deaf community events and activities. We can therefore account for the lower score for this test item after the definition was included by hypothesising that the mention of 'Deaf sports' in the definition acted as a distractor and encouraged learners to assume that the test item was correct.

From this brief review of possible causes of learner errors persisting after definitions were added, it is likely that these causes included flaws in the test instrument as well as test participants not making use of the information provided in the definitions. Further evidence for this second cause comes from the fact that for three of the items in Test 2 with error correction scores below 50\%, the relevant information was not only included in the definition but was also present in other parts of the dictionary entry. For sign BC 5 SMALL-ROUND-FOODPLURAL, for example, the error consisted of a substitution of the word 'potato' as a possible referent instead of 'peas' or 'beans'. Information in the dictionary entry that would exclude potatoes included the reference in the English equivalent and the definition alike to 'small' food items, along with the fact that potatoes are not mentioned in the long list of secondary glosses, whereas 'peas' and 'beans' are. Further relevant information is found in the mouth pattern 'pea' in the definition, and the explanation that the sign refers to food items that you can pop into your mouth one by one. For sign DP 5 DECREASE, the relevant exemplification was included in the example sentences as well as in the definition. For sign EP2 SPEECH, the secondary glosses and a usage note provided relevant information. It seems, then, that the definitions, both by themselves and together with other parts of the dictionary entry, largely contained relevant information, but that this information was not, or could not be, accessed by the intermediate language learners who took the test. 


\section{Feedback from test participants}

To complete the evaluation of the pilot dictionary definitions, I invited feedback from a small group of test participants immediately after the test was administered. Before asking for their opinions on the pilot definitions, I first asked the group about their experiences of taking the test, their use of the dictionary information during the test and how it compared with their dictionary use at other times.

Responses confirmed that when participants were already familiar with the target sign, they did not always look at the dictionary information in the test item even though they had been directed to do so. In common with the findings about how the ODNZSL is mostly used (see section 4.6.3.4 - Information viewed in the dictionary entry), test participants reported that they looked at the English equivalents and the video showing how the sign is produced, but rarely looked at the other information in the dictionary entry. Example sentences were sometimes used in the production part of the test when participants were uncertain about grammatical issues or about how an unfamiliar sign was used in context; but more commonly, participants indicated that they relied on their own developing intuitions about sign usage. Reading the English gloss transcription and the English translation of the example sentence was usually sufficient and the example video itself was therefore often not viewed.

Participants noted issues with finding the information they needed, both in terms of locating alternative signs when correcting errors in the production section, and in terms of identifying an example that matched the context of the test item when there were multiple dictionary entries and examples to be considered. Participants' comments again confirmed that in the immediate lookup situation, the right information has to be found quickly or else it is ignored.

Feedback on the test instrument showed that the difficulty level was perceived as being appropriate for intermediate NZSL learners. The comprehension section was found to be easier to answer than the production section. Most of the signs used in the test items were familiar, but occasionally the dictionary information for these signs was consulted to confirm usage. Participants therefore liked the ability to immediately look up unfamiliar signs in the test sentences by clicking on the still image of the sign. 
The pilot definitions were also reported to be generally comprehensible, although occasional difficulties were noted. For example, one participant stated that:

"Occasionally I would have to watch it twice, or I had to really concentrate to figure out what she was actually saying, because there was no English translation by the side."

However, on reflection, this participant and others in the group stated that a translation into English would not be helpful to them, since the value in the definitions lay in their demonstration of NZSL features in the context of the explanation. Participants mentioned that it would be more confusing than helpful to read an English translation. It was also felt that the more in-depth information in the signed definitions would only be of use to learners at an intermediate or higher level.

The speed of the videos $(80-85 \%$ of the actual signing speed) was found to be good.

When comparing the pilot definitions with the example videos that are currently in the ODNZSL, all participants stated that they liked the definitions. Comments included:

- “nice to see the description.”

- " put the sign in context."

- "Lots of different options are good. Different ways you can say it."

- " "It gave me a deeper understanding of the word I think."

The contextualised presentation of features such as mouth patterns and inflections, and the integrated examples of use, were the most helpful aspects of the definitions. One participant observed in relation to the definition for sign $\mathrm{BC}_{5}$ SMALL-ROUND-FOOD-PLURAL containing examples of combining the sign with different mouthings:

“... I think that was helpful. It was exactly like reading the definition on the website, because it said: this could be used for grapes, nuts, etc. But I think seeing her do it, it just made it more clear, like how it could be used in a visual way. (...)"

Participants reported that during the test, several definitions had provided them with information that was directly relevant because it provided an example or a counterexample that helped them determine whether a test item was correct or not. All participants said they would use definition videos if they were available, in addition to their current use of example sentences. 
In summary, participants' perceptions of the pilot definitions were very positive, both in terms of their content and their presentation. However, test results and dictionary use in practice suggest that the perceived usefulness of signed definitions does not translate into practical benefits during lookups for communicative (comprehension and production) tasks.

\subsection{Conclusion}

This chapter has focused on the practical application and evaluation of the folk definitionbased model for signed dictionary definitions put forward in this thesis. In the first section of this chapter, it was shown that the core characteristics of folk definitions as informal contextual explanations were valued by focus groups of Deaf NZSL users and that their content was perceived as an authentic reflection of Deaf cultural points of view. Opinions about the format of signed dictionary definitions expressed by the focus group participants were taken into account in the second section, which described a possible method for editing and filming signed dictionary definitions. The twelve pilot definitions that were created to test out this method were shown to meet with the criteria of the focus groups and to retain many of the features that were valued in the folk definitions. However, stylistic differences occurred as a result of the decontextualisation of dictionary definitions and influences on the sign model's language performance due to an awareness of the eventual target audience of hearing sign language learners.

This thesis has taken the viewpoint that the decision whether or not to introduce signed definitions to sign language dictionaries should ultimately be based on user needs. The selection and editing of the pilot definitions was influenced by the examination in previous chapters of the user needs of the largest identified group of sign language dictionary users: hearing sign language learners (in particular, intermediate learners). In the third section of this chapter, the pilot dictionary definitions were evaluated positively by hearing intermediate learners, but testing the definitions' effect on learners' sign language comprehension and production could not establish any statistically significant effect. The test instrument, small sample size and the statistical analysis itself may have been factors in this result. More importantly, feedback from the test participants confirmed evidence from the previous study of users of the ODNZSL (see 4.6.2 - What situations prompt dictionary use?) and observations in the literature on dictionary use (see 5.1.1 - Purposes of dictionary use) that the impact of definitions in communicative situations is limited by dictionary users' consultation behaviour and their ability to locate the relevant information quickly. Feedback 
on the definitions themselves suggested that folk definition-inspired signed definitions were seen as useful and potentially contributing to a deeper understanding of the meaning and use of signs. The results of this section are therefore not wholly discouraging.

The main implication of this chapter is that folk definitions are confirmed to be a suitable basis for guiding the development of signed dictionary definitions, and that it is viable to create such definitions, although the time required to carry out the process of data gathering, editing and re-filming is a considerable barrier. The detailed description in the second section of this chapter of how pilot definitions were created also makes a contribution to sign language lexicography by highlighting both theoretical and practical considerations in this process.

The wider implications of this study for the thesis as a whole will be discussed in the next and final chapter. 


\section{Chapter 8: Conclusion}

\section{Introduction}

Each of the four studies presented in this thesis has highlighted a different aspect of the main research question: Should signed definitions be introduced to sign language dictionaries?

In this concluding chapter I will review the main findings of the individual studies and examine how these findings provide an answer to the research question. I will also describe the original contributions made by this thesis, its limitations, and possible future research directions, before discussing the implications of the research findings for sign language lexicography.

\subsection{Summary of research findings}

In the introduction to this thesis, I outlined a number of reasons that have been put forward in the literature for considering the addition of monolingual information, including signed definitions, to sign language dictionaries. These reasons included the status-enhancing effects of (monolingual) dictionaries and a need for documenting sign languages in a way that originates from Deaf people's language use and cultural knowledge, as well as the possible benefits signed definitions might have for dictionary users.

Chapters 2 and 3 gave an overview of the factors that have until recently prevented the inclusion of signed definitions in sign language dictionaries. In these chapters I also described the position of sign languages as minority languages experiencing simultaneous expansion and threats to their vitality. The conclusion of the literature review was that although we are now able to overcome some of the previous obstacles to the inclusion of definitions, there are still considerable challenges. The current state of sign language lexicography has led to dictionaries that are mainly bilingual in format and multifunctional, due in part to the limited resources available for dictionary making.

With this background in mind, the thesis sought to answer the question whether signed definitions should be added to sign language dictionaries by undertaking a user-focused case study of a representative generic (non-technical) sign language dictionary, the ODNZSL. Four separate studies were carried out. 
The first study, reported in Chapter 4, acted as a preliminary to the main research question and asked which user needs signed definitions would fulfil. The study used log file data and a Think Aloud Protocol to examine how the ODNZSL is currently used. The main findings of the study were that the most frequent and most in-depth users of this dictionary were beginner and intermediate language learners involved in formal sign language learning classes. By far the most frequent communicative user situation for this group was language production. This user group was also found to use the ODNZSL in cognitive situations, including vocabulary learning. Particularly relevant to the overall research question were the findings that much of the dictionary information available was not viewed in communicative situations, and that users tended to treat the dictionary as a generic internet search tool, for example by searching for whole phrases, inflected forms, and proper names. These user behaviours implied that the addition of new information might not resolve some of the issues users experience, and that these issues should be addressed instead through adjustments to the user interface, customisation of information, and training users in dictionary use. Having identified hearing, intermediate language learners as the main user group likely to benefit from signed definitions, the second study (Chapter 5) reviewed the current content of the ODNZSL to identify categories of lexical items that were potentially problematic for this user group. An error correction test with comprehension and production components established that only a small number of the tested lexical items were in fact problematic, with $80 \%$ of the 50 items answered correctly by more than half of the test participants. No statistically significant predictors for problematic items were found. However, a qualitative examination of test responses found that intermediate language learners experienced difficulties with some aspects of NZSL grammar, usage and variation. These difficulties were not resolved by test participants' consultation of the ODNZSL due to three factors: firstly, the information required to correct errors was not always present in the dictionary; secondly, in a few cases, glosses and examples may have been misleading or distracting; and thirdly, even when the information was contained in the entry, it was not always located or used. The study concluded that signed definitions might contribute to an improvement in the first two of these factors.

The next step towards answering the overall research question was to select a suitable model for creating signed definitions. An extensive rationale was provided in Chapter 6 for the choice of folk definitions as a model, based on current practice in (pedagogical) lexicography and the particular context of sign language dictionaries. 
The third study examined the content and form of elicited folk definitions by Deaf NZSL users. The main findings were that a number of semantic relationships found in folk definitions of spoken languages, including synonymy, exemplification, comparison, and attribution, were also commonly used to explain the meaning of signs. Correspondences were found between certain sign categories and the kind of semantic relationship used in the folk definition. The signed folk definitions were also found to embed grammatical, situational and sociolinguistic information, although observations about sociolinguistic variation did not always reflect actual variation in NZSL. Stereotypical connotations referred to in the folk definitions reflected the cultural values and behaviours of Deaf NZSL users. These findings provided a compelling case for using folk definitions to gather rich data on the semantics and usage of lexical signs, to supplement current sign language corpus developments. Moreover, this study identified a number of lexical signals and syntactic and grammatical patterns that might form the basis of a signed dictionary definition format. No conclusion was reached as to how the complex relationship between signs and mouth patterns of English words should be dealt with in signed dictionary definitions, but the inclusion of fingerspelled equivalents in the definitions was discouraged.

In the fourth study (Chapter 7), a set of pilot signed dictionary definitions was created based on the findings from the third study and an evaluation of definition formats by Deaf NZSL users. It was possible to retain several important features of both the content and form of folk definitions in the final dictionary definition format. These features included an informal style, an authentic Deaf point of view, and embedded contextual examples and grammar information. The filming process was found to influence sign language production in the definitions towards patterns that were less typical of informal conversational use. A test of the effectiveness of the pilot definitions using the test instrument developed in the second study showed no significant improvements in learners' error correction and identification scores. The overall finding of this study was that positive evaluations of the pilot dictionary definitions by both Deaf L1 sign language users and hearing L2 learners confirmed the viability of the folk definition format as a model, but that the definitions were not directly useful to learners in communicative (comprehension and production) tasks. 


\subsection{Should signed definitions be added to sign language dictionaries?}

Together, the four studies have shown that signed definitions do not meet the main user need of the largest user group. These results therefore strongly suggest that the answer to the main research question should be negative, especially in light of the time- and resource-intensive process of constructing signed definitions. However, this conclusion does not detract from the importance of working towards the use of sign languages as dictionary metalanguages and continuing to privilege Deaf people's perspectives wherever possible.

Moreover, the results of the fourth study suggested that the cognitive user needs of intermediate hearing L2 learners of a sign language, including vocabulary learning, can be met by a folk definition-inspired format, containing relevant information in a comprehensible form. The literature on the role of definitions in vocabulary learning (e.g. Nation, 2001) indicated that the impact of individual dictionary lookups was likely to be very small and it has therefore not been possible to measure any potential benefits using the small sample of pilot definitions in this thesis. Nevertheless, the confirmation that signed definitions were perceived as useful and contributing to L2 learners' depth of vocabulary knowledge suggests that there may be a case for including signed definitions selectively for some lexical items when resources allow. Further research is required to target those entries that would benefit most from this treatment, since no category of lexical items was established to be more problematic than other categories. Brien and Turner's (1994) arguments for focusing initially on the most culture-bound and idiomatic parts of the lexicon remain a useful starting point.

\subsection{Original contributions}

The multifaceted applied research presented in this thesis makes several original contributions, primarily to the field of sign language lexicography but also to the broader areas of sign language linguistics and general lexicography.

A major contribution of the thesis is the application of user-focused (meta-)lexicographical theory, in particular the theory of lexicographic functions (e.g. Tarp, 2009) to sign language dictionaries. Although it had previously been argued that this approach would be beneficial (Kristoffersen \& Troelsgård, 2012), to my knowledge the present thesis is the first comprehensive, empirical, user-focused study to have been carried out on a sign language dictionary. 
A particular strength of the thesis is its careful selection of complementary methods to triangulate results. Log file analysis, itself a relatively recent method in lexicographical studies, had not previously been applied to a sign language dictionary. This study not only objectively confirmed the findings from previous (subjective) user surveys, but also offered new insights into dictionary user behaviour. The findings from the log file analysis, complemented by observed user behaviour from the Think Aloud Protocol, have immediate practical applications and have already contributed to changes in the user interface and content of the ODNZSL, including the decision to make the most viewed part of the dictionary entry - the video demonstrating how the sign is produced—playable on the search results page, rather than requiring dictionary users to click through to individual entries. Beyond these immediate applications, this study also contributes data on users' interactions with multimedia elements and online interfaces that can inform new developments in general digital lexicography.

The thesis has also highlighted the challenges faced by lexicographers of minority and endangered languages in seeking to apply a user-focused theoretical model. In addition to the resource constraints that often prevent a diversification in formats to meet the needs of different user groups, it is also difficult at times to reconcile the learning needs and expectations of majority groups with the desire to preserve and strengthen the minority language. In light of these challenges, the largest contribution of this thesis is that it has proven the viability of a folk definition model as a method for gathering data and creating comprehensible signed dictionary definitions. The folk definitions-based methodology proposed by this thesis accommodates the multiple goals of sign language dictionaries, foregrounds Deaf sign language users' perspectives, adds a level of objectivity to previous practices in developing signed definitions, and complements other developments such as the increasing use of sign language corpora. The description of the steps involved in creating pilot dictionary definitions in the fourth study makes a further practical contribution to sign language lexicography.

Finally, the analysis of folk definitions presented in this thesis expands the semantic description of the NZSL lexicon to date and many of the findings may be extendable to other sign languages given commonalities in the structure of the signed lexicon. 


\subsection{Limitations and future research}

The limitations of the four individual studies have been reported in the respective chapters.

Overall, the main limitation of the thesis is its small sample sizes. The modest size of the documented lexicon and much smaller numbers of users of minority and endangered language dictionaries, compared to the majority language dictionaries that have been the subject of much lexicographical research, place limits on the research methods that can be applied effectively. It would be beneficial to confirm the findings of this research with data from other sign languages and sign language dictionaries.

The development of a computer-based error correction test instrument showed that while it is possible to gather experimental data, the amount of time and effort involved in the construction of such instruments may not be warranted given the difficulties of obtaining sufficient data for a robust statistical analysis. In the New Zealand context, it is unlikely that larger sample sizes could be obtained; however, there may be scope for a similar quantitative study using material from a sign language dictionary in a country with a larger population of (L1 and L2) sign language users.

The thesis has only evaluated the user needs of the main user group, i.e. hearing L2 learners of a sign language. As was indicated in Chapter 4, Deaf L1 sign language users may well have unmet dictionary user needs that include a potential need for descriptions of sign meaning in their first language. However, the studies in this thesis have not contributed any further data to establish whether these user needs exist. The Think Aloud Protocol study provided some insight into the dictionary user behaviour of a few Deaf individuals, but larger-scale usability studies with this user group are required.

This thesis is placed at the nexus of several rapidly developing fields, and a number of technological and methodological changes have taken place since I started out on this research. In terms of methodology, new developments such as eye tracking studies and more extensive log file analysis capabilities now offer possibilities to observe users' interactions with online dictionaries in greater detail. Some of the features of the sign language dictionaries described in this thesis have changed and a number of new online sign language dictionaries have appeared. To my knowledge, signed definitions have still not appeared outside of a small number of technical sign language dictionaries. 
New technical developments are also rapidly giving rise to different tools that complement sign language dictionaries, for example Word-Net-like lexical databases such as ASL-Lex (Caselli, Sevcikova, Cohen-Goldberg, and Emmorey, 2016; http://www.asl-lex.org), video user feedback mechanisms (e.g. DGS-feedback: https://www.sign-lang.uni-hamburg.de/dgskorpus/files/inhalt_pdf/flyer_dgsfeedback.pdf) and several experiments with sign avatars. Such developments have been out of the scope of the current thesis, but they are likely to impact significantly on dictionary user behaviour in the future. It is important to continue to assess the usability of these new tools.

A final area for future research that has not been addressed in detail in this thesis is the area of vocabulary learning in relation to the 'non-core' (productive) lexicon of sign languages; in particular, how the iconic and metaphorical properties of this part of the lexicon could be presented to learners to facilitate learning, and whether sign language dictionaries should have a role in this or whether they should be restricted to describing the core, established lexicon.

\subsection{Implications for sign language lexicography}

The findings of this thesis have implications for two main areas in sign language lexicography. The first area concerns gathering data about the sign language lexicon in preparation for creating a dictionary entry, and the second area involves decisions around how information is presented to users.

With regard to the first area, it has already been mentioned that the research presented here strongly supports the elicitation of signed folk definitions as a data gathering method. In the case of existing dictionary projects, elicited folk definitions could be added to the lexical database to provide more detailed insights into the semantics and usage of the lexicon covered in the dictionary, to verify whether the current dictionary entry is complete and typical, and to identify any gaps in coverage.

At present, many sign language corpora are at least partly based on elicited information, for example elicitation via a list of common concepts, or retellings of well-known narratives. Introducing a further simple elicitation task by asking participants to explain the meaning of a list of signs to a conversation partner would be an immediate way to expand the usefulness of corpora for semantic analysis, as a complement to spontaneous conversational data. 
Eliciting folk definitions will also be a useful method in field work on previously undocumented sign languages, not only because this method reduces the influence of the lexicographer's own (spoken and/or signed) language in the analysis, but also because the direct involvement of native language users in a dictionary project itself contributes to strengthening and maintaining the language (Mosel, 2011).

In relation to the second area, the findings from the thesis recommend a selective approach to introducing signed definitions to generic sign language dictionaries. Although the thesis has suggested a practical methodology for editing and filming signed definitions, in most cases a more urgent priority will be to explore adjustments that can be made to the dictionary user interface to better meet the communicative needs of dictionary users.

Ongoing monitoring of dictionary use (primarily through log files) and providing dictionary users with training in how to interact with the dictionary will prove to be essential factors in the overall usefulness of sign language dictionaries.

\subsection{Conclusion}

The main purpose of this thesis was to establish whether sign language lexicography should take advantage of the possibilities offered by the electronic dictionary medium by including signed definitions as an alternative or addition to the current provision of bilingual equivalents. This question was investigated through an empirical study of user needs, combining several quantitative and qualitative methods within the user-focused framework of the theory of lexicographic functions. Although the research findings did not support an investment of lexicographical resources into creating signed dictionary definitions in the current context, since they do not meet the main user needs of the largest user group, this research has provided a clearer understanding of the potential (if limited) roles that signed definitions can fulfil for L2 learners of a sign language. In addition, the thesis has shown the value of elicited folk definitions as a rich source of semantic and usage data and as a template for dictionary definitions. Since this study breaks new ground in several respects, it is hoped that the suggested user-focused methodology, which seeks to strike a balance between practical resource constraints and ideological principles of language maintenance and promotion, will be expanded on by future research. 


\section{References}

\section{Dictionaries and glossaries}

Boyes Braem, P. (Ed.). (2009). Gebärden-Fachlexikon Deutschschweizerische Gebärdensprache / Deutsch. Retrieved from http://www.signlang.ch

Brien, D. (Ed.). (1992). Dictionary of British Sign Language / English. London: Faber and Faber. $[D B S L / E]$

Brien, D., Brennan, M., Collins, J., Reed, M., \& Thoutenhoofd, E. D. (1997). BSL CD-ROM Dictionary of Deaf Community and Culture. Durham / Breukelen: Deaf Studies Research Unit, University of Durham / BSL Computer Consultancy.

Center for Tegnsprog. (2008). Ordbog over Dansk Tegnsprog (Danish Sign Language Dictionary). Copenhagen: Center for Tegnsprog. Retrieved from http:// www.tegnsprog.dk

Deverson, T., \& Kennedy, G. (Eds.). (2005). The New Zealand Oxford Dictionary. Oxford: Oxford University Press.

Eesti Keele Instituut. (n.d.). Eesti Viipekeele - Eesti Keele Sõnastiku (Estonian Sign Language -Estonian Online Dictionary). (n.d.). Tallinn: Eesti Keele Instituut. Retrieved from http://www.eki.ee/dict/viipekeel/

FDMSE (Faculty of Disability Management and Special Education). (n.d.). Indian Sign Language Dictionary. Coimbatore: Ramakrishna Mission Vivekananda University. Retrieved from http://indiansignlanguage.org/dictionary

Fenlon, J., Cormier, K., Rentelis, R., Schembri, A., Rowley, K., Adam, R., \& Woll, B. (2014). BSL SignBank: A lexical database of British Sign Language (First edit.). London: Deafness, Cognition and Language Research Centre, University College London. Retrieved from http://bslsignbank.ucl.ac.uk/

Hewgill, G. (2013). NZSL dictionary for iPad / iPhone / iPod Touch and Android [Computer software]. Retrieved from https://hewgill.com/nzsldict/

Hornby, A. S., \& Cowie, A. P. (Eds.). (1989). Oxford Advanced Learner's Dictionary of Current English (Fourth edit.). Oxford: Oxford University Press.

Johnston, T. A. (1989 a). Auslan Dictionary: A dictionary of the sign language of the Australian Deaf community. Sydney: Deafness Resources Australia.

Johnston, T. A. (Ed.). (2014). AUSLAN SignBank. Sydney: Macquarie University. Retrieved from http://www.auslan.org.au/

Kennedy, G. D., Arnold, R., Fahey, S., \& Moskovitz, D. (Eds.). (1997). A Dictionary of New Zealand Sign Language. Auckland: Auckland University Press with Bridget Williams Books.

Kennedy, G. D., McKee, D., Arnold, R., Dugdale, P., Fahey, S., \& Moskovitz, D. (Eds.). (2002). A Concise Dictionary of New Zealand Sign Language. Wellington: Bridget Williams Books. 
Konrad, R., Langer, G., König, S., Schwarz, A., Hanke, T., \& Prillwitz, S. (Eds.). (2007). Fachgebärdenlexikon Gesundheit und Pflege. Hamburg: University of Hamburg. Retrieved from http://www.sign-lang.uni-hamburg.de/glex/intro/inhalt.htm

Kuurojen Liitto (Finnish Association of the Deaf). (2003). Suvi Suomalaisen viittomakielen verkosanakirja. (Finnish Sign Language Dictionary). Helsinki: Kuurojen Liitto. Retrieved from http://suvi.viittomat.net

Levitt, D. (1986). Introduction to New Zealand Sign Language. Auckland: National Foundation of the Deaf.

McKee, D., McKee, R., Pivac Alexander, S., Pivac, L., \& Vale, M. (2011). Online Dictionary of New Zealand Sign Language. Wellington: Deaf Studies Research Unit, Victoria University of Wellington. Retrieved from http://nzsl.vuw.ac.nz [ODNZSL]

Müller, L. (Ed.). (n.d.). Online slovník českého znakového jazyka (Online Dictionary of Czech Sign Language). Plzen: University of West Bohemia in Pilsen. Retrieved from: http:// znaky.zcu.cz

Rundell, M., \& Fox, G. (2002). MacMillan English Dictionary for Advanced Learners. Oxford: Macmillan.

Schermer, G. M. M., Harder, R., \& Bos, H. (1988). Handen uit de mouwen: Gebaren uit de Nederlandse Gebarentaal in kaart gebracht. Amsterdam: NSDSK/Dovenraad.

Schermer, G. M. M., Koolhof, C., Muller, S., Geuze, J., \& Vink, M. (Eds.). (2009). Van Dale Basiswoordenboek Nederlandse Gebarentaal. Utrecht / Bunnik: Van Dale / Nederlands Gebarencentrum.

Scottish Sensory Centre. (n.d.). British Sign Language Glossaries of Curriculum Terms. Edinburgh: Scottish Sensory Centre. Retrieved from http://www.ssc.education.ed.ac.uk/ BSL/list.html

Shaw, E., \& Delaporte, Y. (2014). A Historical and Etymological Dictionary of American Sign Language. Washington, D.C.: Gallaudet University Press.

Sinclair, J. M. (Ed.). (1987 a). Collins COBUILD English Language Dictionary (First Edit.). London / Glasgow: Collins.

Sternberg, M. L. A. (1996). The American Sign Language Dictionary on CD-ROM (version 2.). New York: HarperCollins.

Stokoe, W. C., Casterline, D. C., \& Croneberg, C. G. (1965). A Dictionary of American Sign Language on Linguistic Principles (First edit.). Washington, D.C.: Gallaudet College Press. [DASL]

Summers, D. (Ed.). (1987). Longman Dictionary of Contemporary English (Second edit.). Harlow, Essex: Longman.

Suwanarat, M., Ratanasint, A., Rungsrithong, V., Anderson, L., \& Wrigley, O. P. (Eds.). (1990). The Thai Sign Language Dictionary. Bangkok: National Association of the Deaf in Thailand. 
University of Wolverhampton. (n.d.). ArtSigns: The Online BSL/English Glossary for Art and Design. Retrieved from http://www.artsigns.ac.uk

van Herreweghe, M., Slembrouck, S., \& Vermeerbergen, M. (Eds.). (2004). Digitaal Vlaamse Gebarentaal - Nederlands / Nederlands - Vlaamse Gebarentaal Woordenboek. Retrieved from http://gebaren.ugent.be 


\section{Other references}

Adamska-Salaciak, A. (2010). Examining equivalence. International Journal of Lexicography, 23(4), 387-410.

Adamska-Salaciak, A. (2013). Equivalence, synonymy, and sameness of meaning in a bilingual dictionary. International Journal of Lexicography, 26(3), 329-345.

Ahlgren, M. (1986). New Zealand Sign Language and the aim of Total Communication for the deaf. SET: Research information for teachers. Wellington: New Zealand Council for Educational Research.

Aitchison, J. (1987). Words in the mind. Oxford: Blackwell.

Al-Ajmi, H. (2008). The effectiveness of dictionary examples in decoding: The case of Kuwaiti learners of English. Lexikos, 18(May 2007), 15-26.

Atkins, B. T. S., \& Knowles, F. E. (1990). Interim report on the EURALEX/AILA research project into dictionary use. In T. Magay \& J. Zigány (Eds.), Budalex '88 Proceedings (pp. 381-392). Budapest: Akadémiai Kiadó.

Atkins, B. T. S., \& Rundell, M. (2008). The Oxford guide to practical lexicography. Oxford: Oxford University Press.

Atkins, B. T. S., \& Varantola, K. (1997). Monitoring dictionary use. International Journal of Lexicography 10(1), 1-45.

Bank, R., Crasborn, O., \& van Hout, R. (2011). Variation in mouth actions with manual signs in Sign Language of the Netherlands (NGT). Sign Language E Linguistics, 14(2), 248-270.

Barnbrook, G. (2002). Defining language: A local grammar of definition sentences. Amsterdam / Philadelphia: John Benjamins.

Battison, R. (1978). Lexical borrowing in American Sign Language. Silver Spring, Maryland: Linstok Press.

Béjoint, H. (2000). Modern lexicography: An introduction. Oxford: Oxford University Press.

Bell, A. (1991). Audience accommodation in the mass media. In H. Giles, J. Coupland, \& N. Coupland (Eds.), Contexts of accommodation: Developments in applied sociolinguistics (pp. 69-102). Cambridge: Cambridge University Press.

Bellugi, U., \& Fischer, S. (1972). A comparison of signed and spoken language. Cognition, $1(2-3), 173-200$.

Bergenholtz, H., \& Johnsen, M. (2007). Log files can and should be prepared for a functionalistic approach. Lexikos, 17, 1-20.

Bergenholtz, H., \& Tarp, S. (2003). Two opposing theories: On H.E. Wiegand's recent discovery of lexicographic functions. Hermes Journal of Linguistics, 31, 171-196.

Bickford, J. A., Lewis, M. P., \& Simons, G. F. (2015). Rating the vitality of sign languages. Journal of Multilingual and Multicultural Development, 36(5), 513-527. 
Bienvenu, M. (2003). Developing a prototype for a monolingual ASL dictionary. (Unpublished doctoral dissertation). Union Institute and University, Cincinnati.

Boyes Braem, P. (2001). Functions of the mouthings in the signing of Deaf early and late learners of Swiss German Sign Language (DSGS). In P. Boyes Braem \& R. Sutton-Spence (Eds.), The hands are the head of the mouth: The mouth as articulator in sign languages (pp. 99-132). Hamburg: Signum-Verlag.

Boyes Braem, P., Pizzuto, E., Volterra, V., Schulmeister, R., \& Reinitzer, H. (2002). The interpretation of signs by (hearing and deaf) members of different cultures. In R. Schulmeister \& H. Reinitzer (Eds.), Progress in sign language research: In honor of Siegmund Prillwitz (pp. 187-219). Hamburg: Signum-Verlag.

Boyes Braem, P., \& Sutton-Spence, R. (Eds.). (2001). The hands are the head of the mouth: The mouth as articulator in sign languages. Hamburg: Signum-Verlag.

Branson, J., \& Miller, D. (1997). National sign languages and language policies. In (N. H. Hornberger, Ed.) Encyclopedia of Language and Education Volume 1: Language policy and political issues in education. Houten: Springer

Brennan, M. (1990). Word formation in British Sign Language. Stockholm: University of Stockholm.

Brennan, M. (1992). The visual world of BSL: An introduction. In D. Brien (Ed.), Dictionary of British Sign Language/English. London: Faber and Faber.

Brennan, M. (2001). Making borrowings work in British Sign Language. In D. Brentari (Ed.), Foreign vocabulary in sign languages: A cross-linguistic investigation of word formation (pp 49-85). Mahwah, New Jersey: Lawrence Erlbaum Associates.

Brennan, M. (2005). Conjoining word and image in British Sign Language (BSL): An exploration of metaphorical signs in BSL. Sign Language Studies, 5(3), 360-382.

Brentari, D., \& Padden, C. A. (2001). Native and foreign vocabulary in American Sign Language: A lexicon with multiple origins. In D. Brentari (Ed.), Foreign vocabulary in sign languages: A cross-linguistic investigation of word formation (pp. 87-120). Mahwah, New Jersey: Lawrence Erlbaum Associates.

Brien, D., \& Collins, J. (1997). Some issues in the creation of sign language dictionaries. Paper presented at Empower '97: International Conference on Deaf Education, Scottish Sensory Centre, Edinburgh. Retrieved from http://www.ssc.education.ed.ac.uk/ resources/deaf/briencollins.html

Brien, D., \& Turner, G. H. (1994). Lemmas, dilemmas and lexicographical anisomorphism: Presenting meaning in the first BSL-English dictionary. In I. Ahlgren, B. Bergman, \& M. Brennan (Eds.), Perspectives on sign language: Papers from the fifth international symposium on sign language research, Salamanca, Spain, 25-30 May 1992. Volume 2: Perspectives on sign language usage (pp. 391 - 408). Durham, England: International Sign Linguistics Association / Deaf Studies Research Unit, University of Durham.

Campbell, R., Martin, P., \& White, T. (1992). Forced choice recognition of sign in novice learners of British Sign Language. Applied Linguistics, 13(2), 185-201. 
Carmel, S. J. (1992). A checklist of dictionaries of national sign languages of deaf people. Sign Language Studies, 76(3), 233-252.

Carter, R., \& McCarthy, M. (1988). Developments in the teaching of vocabulary. In R. Carter \& M. McCarthy (Eds.), Vocabulary and language teaching (pp. 39 - 59). London / New York: Longman.

Casagrande, J. B., \& Hale, K. L. (1967). Semantic relationships in Papago folk-definitions. In Studies in Southwestern Ethnolinguistics: Meaning and History in the languages of the American Southwest (pp. 165-193). The Hague: Mouton.

Caselli, N. K., Sevcikova, Z., Cohen-Goldberg, A. M., \& Emmorey, K. (2016). ASL-Lex: A lexical database for ASL. Behavior Research Methods, 1-18. http://doi.org/10.3758/ s13428-016-0742-o

Chaudron, C. (1982). Vocabulary elaboration in teachers' speech to L2 learners. Studies in Second Language Acquisition, 4(2), 170-180.

Collins-Ahlgren, M. (1989). Aspects of New Zealand Sign Language. (Unpublished doctoral dissertation). Victoria University of Wellington, Wellington, New Zealand.

Cormier, K., Schembri, A., \& Woll, B. (2013). Pronouns and pointing in sign languages. Lingua, 137, 230-247.

Corris, M., Manning, C., Poetsch, S., \& Simpson, J. (2002). Dictionaries and endangered languages. In D. Bradley \& M. Bradley (Eds.), Language endangerment and language maintenance (pp. 329 - 347). London / New York: Routledge Curzon.

Cowie, A. P. (1989). The language of examples in English learners' dictionaries. In G. James (Ed.), Lexicographers and their works (pp. 55-65). Exeter: University of Exeter.

Crasborn, O., van der Hulst, H., \& van der Kooij, E. (2001). SignPhon: A phonological database for sign languages. Sign Language $\mathcal{E}$ Linguistics. doi:10.1075/sll.4.12.15cra

Crasborn, O., van der Kooij, E., Waters, D., Woll, B., \& Mesch, J. (2008). Frequency distribution and spreading behavior of different types of mouth actions in three sign languages. Sign Language and Linguistics, 11(1), 45- 67.

Cuxac, C. (1996). Fonctions et structures de l'iconicité des langues des signes. Analyse descriptive d'un idiolecte parisien de la langue des signes française. (Unpublished doctoral dissertation). Paris V, Paris, France.

De Schryver, G.-M. (2003). Lexicographers' dreams in the electronic dictionary age. International Journal of Lexicography, 16(2), 143-199.

De Schryver, G.-M., \& Joffe, D. (2004). On how electronic dictionaries are really used. In Proceedings of the eleventh EURALEX International Congress, (pp. 187-196). Lorient: Université de Bretagne-Sud.

Deaf Aotearoa New Zealand (2011). New to New Zealand. [Brochure]. Retrieved from http:// www.deaf.org.nz/images/Downloads/New_to_New_Zealand_v5.pdf

Demey, E., van Herreweghe, M., \& Vermeerbergen, M. (2008). Iconicity in sign languages. In K. Willems \& L. de Cuypere (Eds.), Naturalness and iconicity in language (pp. 189-214). Amsterdam: John Benjamins Publishing Company. 
DGS-feedback flyer. (n.d.). Retrieved from https://www .sign-lang.uni-hamburg.de/dgs-korpus/files/ inhalt_pdf/flyer_dgsfeedback.pdf

Dingemanse, M. (2015). Folk definitions in linguistic fieldwork. In J. Essegbey, B. Henderson, \& F. McLaughlin (Eds.), Language documentation and endangerment in Africa (pp. 215238). Amsterdam: John Benjamins Publishing Company.

Duval, A. (2008). Equivalence in bilingual dictionaries. In T. Fontenelle (Ed.), Practical lexicography: A reader (pp. 273 - 282). Oxford: Oxford University Press.

Ebbinghaus, H., \& Hessman, J. (2001). Sign language as multidimensional communication: Why manual signs, mouthings, and mouth gestures are three different things. In P. Boyes Braem \& R. Sutton-Spence (Eds.), The hands are the head of the mouth: The mouth as articulator in sign languages (pp. 133 -152). Hamburg: Signum-Verlag.

Emmorey, K. (2002). Language, cognition, and the brain: Insights from sign language research. Mahwah, New Jersey: Lawrence Erlbaum Associates.

Emmorey, K., Borinstein, H. B., \& Thompson, R. (2005). Bimodal bilingualism: Codeblending between spoken English and American Sign Language. In J. Cohen, K. T. McAlister, K. Rolstad, \& J. MacSwan (Eds.), ISB4: Proceedings of the 4 th International Symposium on Bilingualism (pp. 663-673). Somerville, MA: Cascadilla Press.

Engberg-Pedersen, E. (1993). Space in Danish Sign Language: The semantics and morphosyntax of the use of space in a visual language. Hamburg: Signum Press.

Engberg-Pedersen, E. (1994). Some simultaneous constructions in Danish Sign Language. In M. Brennan \& G. H. Turner (Eds.), Word order issues in sign language: Working papers presented at a workshop held in Durham, 18-22 September 1991 (pp. 73 - 88). Durham: International Sign Linguistics Association.

Fenlon, J., Cormier, K., \& Schembri, A. (2015). Building BSL SignBank: The lemma dilemma revisited. International Journal of Lexicography, 28(2), 169-206.

Fenlon, J., Schembri, A., Johnston, T. A., \& Cormier, K. (2015). Documentary and corpus approaches to sign language research. In E. Orfanidou, B. Woll, \& G. Morgan (Eds.), Research methods in sign language studies: A practical guide. London: Wiley Blackwell.

Fillmore, C. (2003). Double-decker definitions: The role of frames in meaning explanations. Sign Language Studies, 3(3), 263-295.

Flowerdew, J. (1992). Definitions in science lectures. Applied Linguistics, 13(2), 202-221.

Fox, G. (1987). The case for examples. In J. M. Sinclair (Ed.), Looking up: An account of the COBUILD project in lexical computing (pp. 137-149). London: Collins ELT.

Frankenberg-Garcia, A. (2012). Learners' use of corpus examples. International Journal of Lexicography, 25(3), 273-296.

Frawley, W., Hill, K. C., \& Munro, P. (Eds.). (2002). Making dictionaries: Preserving indigenous languages of the Americas. Berkeley: University of California Press.

Friedman, L. A. (1975). Space, time, and person reference in American Sign Language. Language, 51(4), 940-961. 
Frishberg, N. (1975). Arbitrariness and iconicity: Historical change in American Sign Language. Language, (51), 696 - 719.

Frishberg, N. (1987). Home sign. In J. V. Van Cleve (Ed.), Gallaudet Encyclopedia of Deaf People and Deafness (Vol. 3, pp. 128-131). New York: McGraw Hill.

Garcia, O., \& Wei, L. (2014). Translanguaging: Language, bilingualism and education. London: Palgrave MacMillan.

Greville, A. (2005). Hearing impaired and deaf people in New Zealand: An update on population numbers and characteristics. Auckland: Greville Consulting. Retrieved from http://www.oticon.org.nz/pdf/hearingimpaireddeafpeoplenzmaro5a.pdf

Groce, N. E. (1985). Everyone here spoke sign language: Hereditary deafness on Martha's Vineyard. Cambridge, Massachusetts: Harvard University Press.

Gumming, G., Cropp, S., \& Sussex, R. (1994). On-line lexical resources for language learners: Assessment of some approaches to word definition. System, 22(3), 369-377.

Halliday, M. A. K., \& Hasan, R. (1976). Cohesion in English. London: Longman.

Hanke, T. (2002). iLex-A tool for sign language lexicography and corpus analysis. In M. Rodriguez González \& C. Paz Suarez Araujo (Eds.), Proceedings of the third International Conference on Language Resources and Evaluation (LREC 2002), Las Palmas de Gran Canaria, Spain. (pp. 923-926). Paris: ELRA.

Hanks, P. (1987). Definitions and explanations. In J. M. Sinclair (Ed.), Looking up: An account of the COBUILD project in lexical computing (pp. $116-136)$. London: Collins ELT.

Hanks, P. (2015). Definition. In P. Durkin (Ed.), Oxford handbook of lexicography (pp. 94129). Oxford: Oxford University Press.

Hartmann, R. R. K. (1983). On theory and practice. In R. R. K. Hartmann (Ed.), Lexicography: Principles and practice (pp. 3-12). London / New York: Academic Press.

Hartmann, R. R. K. (1989). Sociology of the dictionary user: Hypotheses and empirical studies. In F. J. Hausmann, O. Reichmann, H. E. Wiegand, \& L. Zgusta (Eds.), Wörterbücher/Dictionaries/Dictionnaires. An international encyclopedia of lexicography, Vol.1 (pp. 102-111). Berlin: Walter de Gruyter.

Hartmann, R. R. K. (2001). Teaching and researching lexicography. Harlow, Essex: Pearson Education Ltd.

Haualand, H., \& Allen, C. (2009). Deaf people and human rights. Helsinki: World Federation of the Deaf.

Hessman, J., \& Pyfers, L. (2014). Teaching British Sign Language as a second language to Deaf sign language users: Insights from the Signs2Go online course. In D. McKee, R. S. Rosen, \& R. McKee (Eds.), Teaching and learning signed languages (pp. 222-250). Basingstoke / New York: MacMillan Palgrave.

Hiles, L. (2011). Categorising example sentences in dictionaries for research purposes. Lexikos, 21, 298-304. 
Hulstijn, J. H., \& Atkins, B. T. S. (1998). Empirical research on dictionary use in foreignlanguage learning: Survey and discussion. In B. T. S. Atkins (Ed.), Using dictionaries: studies of dictionary use by language learners and translators (pp. 7-19). Tübingen: Max Niemeyer Verlag.

Humblé, P. (1998). The use of authentic, made-up and controlled examples in foreign language dictionaries. In T. Fontenelle, P. Hiligsmann, A. Michiels, A. Moulin, \& S. Theissen (Eds.), Euralex '98 proceedings: Papers submitted to the Eight EURALEX International Congress on Lexicography, Liège, Belgium. Liège: University of Liège.

Humblé, P. (2001). Dictionaries and language learners. Frankfurt am Main: Haag und Herchen.

Humphries, T., \& MacDougall, F. (1999). "Chaining” and other links: Making connections between American Sign Language and English in two types of school settings. Visual Anthropology Review, 15(2), 84-94.

Jackson, H. (2002). Lexicography: An introduction. London: Routledge.

Johnsen, M. (2005). Logfiler som leksikografisk analyseinstrument og hjælpeværktøj. Århus: Handelshøjskolen i Århus.

Johnston, T. A. (1989 b). Auslan: The sign language of the Australian Deaf community. (Unpublished doctoral dissertation). University of Sydney, Sydney, Australia.

Johnston, T. A. (2003). Language standardization and signed language dictionaries. Sign Language Studies, 3(4), 431-468.

Johnston, T. A. (2006). W(h)ither the Deaf community? Population, genetics, and the future of Australian Sign Language. Sign Language Studies, 6(2), 137-173.

Johnston, T. A. (2008). Corpus linguistics and signed languages: No lemmata, no corpus. In O. Crasborn, E. Efthimiou, T. Hanke, E. Thoutenhoofd, \& I. Zwitserlood (Eds.), Construction and exploitation of sign language corpora. Proceedings of the 3 rd workshop on the representation and processing of sign languages. 6th International Conference on Language Resources and Evaluation, LREC 2008, Marrakech. (pp. 82-87). Paris: ELRA.

Johnston, T.A. (2010). From archive to corpus: Transcription and annotation in the creation of signed language corpora. International Journal of Corpus Linguistics, 15(1), 114-129.

Johnston, T. A. (2012). Lexical frequency in sign languages. Journal of Deaf Studies and Deaf Education, 17(2), 163-93.

Johnston, T. A., \& Ferrara, L. (2012). Lexicalization in signed languages: When is an idiom not an idiom? In C. Hart (Ed.), Selected papers from the third UK Cognitive Linguistics Conference (Vol. 1, pp. 229-248). (n.p.): UK-CLA.

Johnston, T. A., \& Schembri, A. (1999). On defining lexeme in a signed language. Sign Language $\mathcal{E}$ Linguistics, 2(2), 115-185.

Johnston, T. A., \& Schembri, A. (2007). Australian Sign Language: An introduction to sign language linguistics. Cambridge: Cambridge University Press. 
Johnston, T., van Roekel, J., \& Schembri, A. (2015). On the conventionalization of mouth actions in Australian Sign Language. Language and Speech. doi:10.1177/0023830915569334

Karlsson, F. (1984). Structure and iconicity in sign language. In F. Loncke, P. Boyes Braem, \& Y. Lebrun (Eds.), Recent research on European sign languages (pp. 149-155). Lisse: Swets and Zeitlinger.

Kendon, A. (2004). Gesture: Visible action as utterance. Cambridge: Cambridge University Press.

Klima, E. S., \& Bellugi, U. (1979). The signs of language. Cambridge, Mass: Harvard University Press.

Konrad, R. (2012). Sign language corpora survey. Hamburg: Institute for German Sign Language and Communication of the Deaf, University of Hamburg. Retrieved from http://www.sign-lang.uni-hamburg.de/dgs-korpus/files/inhalt_pdf/SL-CorporaSurvey_update_2012.pdf

Kristoffersen, J. H. (2010). From utterance to example sentence-a lexicographic approach to exploitation of corpus. Paper presented at the Sign Linguistics Corpora Network (SLCN) Workshop 4: Exploitation, Berlin, 3-4 December 2010. Retrieved from https:// www.ucviden.dk/portal-ucc/files/10415475/SLCN_Berlin_2010_handout.pdf

Kristoffersen, J. H., \& Troelsgård, T. (2012). The electronic lexicographical treatment of sign languages: The Danish Sign Language Dictionary. In S. Granger \& M. Paquot (Eds.), Electronic Lexicography (First Edit., pp. 293-318). Oxford: Oxford University Press.

Kurland, B. F., \& Snow, C. E. (1997). Longitudinal measurement of growth in definitional skill. Journal of Child Language, 24(03), 603-625.

Kwary, D. (2011). Towards a typology of definitions for LSP dictionaries. Journal of English Studies, $9,55-73$.

Kyle, J. G., \& Woll, B. (1987). Sign language: The study of deaf people and their language. Cambridge: Cambridge University Press.

Ladd, P. (2003). Understanding Deaf culture: In search of Deafhood. Bristol: Multilingual Matters.

Lakoff, G., \& Johnson, M. (1980). Metaphors we live by. Chicago: University of Chicago Press.

Lane, H. (1984). When the mind hears: A history of the deaf. New York: Random House.

Lane, H. (1995). Constructions of deafness. Disability \& Society, 10(2), 171 - 190.

Laufer, B. (1992). Corpus-based versus lexicographer examples in comprehension and production of new words. In H. Tommola, K. Varantola, T. Salmi-Tolonen, \& J. Schopp (Eds.), EURALEX' 92 proceedings I-II: Papers submitted to the 5th EURALEX International Congress on Lexicography in Tampere, Finland (pp. 4-9). Tampere: University of Tampere.

Laufer, B., \& Hadar, L. (1997). Assessing the effectiveness of monolingual, bilingual, and "bilingualised" dictionaries in the comprehension and production of new words. The Modern Language Journal, 81(2), 189 - 196. 
Laufer, B., \& Kimmel, M. (1997). Bilingualised dictionaries: How learners really use them. System, 25, 361-369.

Laufer, B., \& Melamed, L. (1994). Monolingual, bilingual and "bilingualised" dictionaries: Which are more effective, for what and for whom? In W. Martin, W. Meijs, M.

Moerland, E. ten Pas, P. van Sterkenburg, \& P. Vossen (Eds.), Euralex 1994 proceedings: Papers submitted to the 6th Euralex International Congress on Lexicography, Amsterdam, The Netherlands (pp. 565 - 576). Amsterdam: Euralex.

Leeson, L., \& Saeed, J. I. (2012). Irish Sign Language: A cognitive linguistic account. Edinburgh: Edinburgh University Press.

Lew, R. (2004). Which dictionary for whom? Receptive use of bilingual, monolingual and semi-bilingual dictionaries by Polish learners of English. Poznań: Motivex.

Lew, R. (2007). Linguistic semantics and lexicography: a troubled relationship. In M. Fabiszak (Ed.), Language and meaning. Cognitive and functional perspectives (pp. 217-224). Frankfurt am Main: Peter Lang.

Lew, R., \& De Schryver, G.-M. (2014). Dictionary users in the digital revolution. International Journal of Lexicography, 27(4), 341-359.

Lew, R., Grzelak, M., \& Leszkowicz, M. (2013). How dictionary users choose senses in bilingual dictionary entries: An eye-tracking study. Lexikos, 23, 228-254.

Liddell, S. K. (1980). American Sign Language syntax. The Hague / New York: Mouton.

Liddell, S. K. (2003). Grammar, gesture and meaning in American Sign Language. Cambridge: Cambridge University Press.

Lucas, C. (2003). The role of variation in lexicography. Sign Language Studies, 3(3), 322-340.

Lucas, C., Bailey, R., Valli, C., Rose, M., \& Wulf, A. (2001). Sociolinguistic variation. In C. Lucas (Ed.), The sociolinguistics of sign languages (pp. $61-111$ ). Cambridge: Cambridge University Press.

Lucas, C., \& Valli, C. (1990). ASL, English, and contact signing. In C. Lucas (Ed.), Sign language research: Theoretical issues (pp. 288 - 307). Washington, D.C.: Gallaudet University Press.

Luppescu, S., \& Day, R. (1993). Reading, dictionaries, and vocabulary learning. Language Learning, 43, 263-287.

Manes, J. (1980). Ways of defining: Folk definitions and the study of semantics. Forum Linguisticum, 5(2), 122-138.

Mathiot, M. (1979). Folk definitions as a tool for the analysis of lexical meaning. In M. Mathiot (Ed.), Ethnolinguistics: Boas, Sapir and Whorf revisited (pp. 121-26o). The Hague: Mouton.

Matthews, P. W., McKee, R., \& McKee, D. (2009). Signed languages, linguistic rights and the standardisation of geographical names. In W. Ahrens, S. Embleton, \& A. Lapierre (Eds.), Names in multi-lingual, multi-cultural and multi-ethnic contact: Proceedings of the 23rd International Congress of Onomastic Sciences. August 17-22, 2008. Toronto: York University. 
Max Planck Institute for Psycholinguistics Language Archiving Technology Group. (2009). ELAN: EUDICO linguistic annotator.[Computer software]. Nijmegen, The Netherlands: Author. Retrieved from https://tla.mpi.nl/tools/tla-tools/elan/

Mayberry, R. (2006). Learning sign language as a second language. In Encyclopedia of Language and Linguistics (Second Edit., pp. 743-746). Amsterdam / London: Elsevier.

Mayberry, R. I., \& Eichen, E. B. (1991). The long-lasting advantage of learning sign language in childhood: Another look at the critical period for language acquisition. Journal of Memory and Language, 3o(4), 486-512.

McKee, D., \& Kennedy, G. D. (2000). A lexical comparison of signs from American, Australian and New Zealand sign languages. In K. Emmorey \& H. Lane (Eds.), The signs of language revisited: An anthology To honor Ursula Bellugi and Edward Klima (pp. 49-76). Mahwah, New Jersey: Lawrence Erlbaum Associates.

McKee, D., \& Kennedy, G. D. (2006). The distribution of signs in New Zealand Sign Language. Sign Language Studies, 6(4), 372-390.

McKee, D., \& Pivac Alexander, S. (2008). NZSL Online Dictionary project 2008-2011: User requirements survey report. Unpublished manuscript, Victoria University of Wellington.

McKee, R. (2007). Hand to mouth: The role of mouthing in NZSL. Across the Board: Australian Association of Sign Language Interpreters (Victoria), 2(4), 3-12.

McKee, R. (2015). New Zealand Sign Language: A reference grammar. Wellington: Bridget Williams Books.

McKee, R. L., \& Manning, V. (2015). Evaluating effects of language recognition on language rights and the vitality of New Zealand Sign Language. Sign Language Studies, 15(4), 473497.

McKee, R., \& McKee, D. (2011). Old signs, new signs, whose signs? Sociolinguistic variation in the NZSL lexicon. Sign Language Studies, 11(4), 485-527.

McKee, R., \& McKee, D. (2013). Making an online dictionary of New Zealand Sign Language. Lexikos, 23, 500-531.

McKee, R., \& McKee, D. (2016, January). Assessing the vitality of NZSL. Paper presented at TISLR12, Theoretical Issues in Sign Language Research Conference, 4-7 January 2016, Melbourne, Australia. Retrieved from http://www.victoria.ac.nz/lals/centres-andinstitutes/dsru/McKee-Vitality-TISLR-2016-FINAL-edit-.pdf

McKee, R., \& Vale, M. (forthcoming). Sign language lexicography. In P. Hanks \& G.-M. de Schryver (Eds.), International handbook of modern lexis and lexicography. Heidelberg: Springer Verlag.

McKeown, M. G. (1993). Creating effective definitions for young word learners. Reading Research Quarterly, 28(1), 16 - 31.

Meir, I. (2012). Word classes and word formation. In R. Pfau, M. Steinbach, \& B. Woll (Eds.), Sign language: An international handbook (pp. 77-112). Berlin: De Gruyter Mouton. 
Mesch, J., \& Wallin, L. (2012). From meaning to signs and back: Lexicography and the Swedish Sign Language Corpus. In LREC (Language Resources and Evaluation Conference) May 27, 2012, Istanbul (pp. 123-126). Paris: ELRA.

Metzger, M. (1995). Constructed dialogue and constructed action in American Sign Language. In C. Lucas (Ed.), Sociolinguistics in Deaf communities (pp. 255-271). Washington, D.C.: Gallaudet University Press.

Miller, C. (1994). Simultaneous constructions in Quebec Sign Language. In M. Brennan \& G. H. Turner (Eds.), Word order issues in sign language: Working papers presented at a workshop held in Durham, 18-22 September 1991 (pp. 89 - 112). Durham: International Sign Linguistics Association.

Minaeva, L. (1992). Dictionary examples: Friends or foes? In H. Tommola, K. Varantola, T. Salmi-Tolonen, \& J. Schopp (Eds.), EURALEX' 92 proceedings I-II: Papers submitted to the 5th EURALEX International Congress on Lexicography in Tampere, Finland. Tampere: University of Tampere.

Mitchell, R. E., \& Karchmer, M. A. (2004). Chasing the mythical ten percent: Parental hearing status of deaf and hard of hearing students in the United States. Sign Language Studies, $4(2), 138-163$.

Morford, J. P., \& MacFarlane, J. (2003). Frequency characteristics of American Sign Language. Sign Language Studies, 3(2), 213-226.

Mosel, U. (2011). Lexicography in endangered language communities. In P. K. Austin \& J. Sallabank (Eds.), The Cambridge handbook of endangered languages (pp. 337-353). Cambridge: Cambridge University Press.

Moskovitz, D. (1994). The Dictionary of New Zealand Sign Language user requirements survey. In I. Ahlgren, B. Bergman, \& M. Brennan (Eds.), Perspectives on sign language: Papers from the fifth international symposium on sign language research: held in Salamanca, Spain, 25-30 May 1992. Volume 2: Perspectives on sign language usage (pp. 421 - 442). Durham, England: International Sign Linguistics Association; Deaf Studies Research Unit, University of Durham.

Murray, J. J. (2015). Special issue: Language planning and sign language rights. Sign Language Studies, 15(4), 375-378.

Nadolske, M. A., \& Rosenstock, R. (2007). Occurrence of mouthings in American Sign Language: A preliminary study. In P. M. Perniss, R. Pfau, \& M. Steinbach (Eds.), Visible variations: Comparative studies on sign language structure (pp. 35 -62). Berlin: Mouton de Gruyter.

Nation, I. S. P. (1989). Dictionaries and language learning. In M. L. Tickoo (Ed.), Learners dictionaries: State of the art (pp. 65-71). Singapore: SEAMEO Regional Language Centre.

Nation, I. S. P. (2001). Learning vocabulary in another language. Cambridge: Cambridge University Press.

National Cued Speech Association (n.d.) About cued speech. Retrieved from http:// www.cuedspeech.org/cued-speech/about-cued-speech.php 
Neese Bailes, C., Erting, C. J., Erting, L. C., \& Thumann-Prezioso, C. (2009). Language and literacy acquisition through parental mediation in American Sign Language. Sign Language Studies, 9(4).

Nesi, H. (1996). The role of illustrative examples in productive dictionary use. Dictionaries: Journal of the Dictionary Society of North America, 17(1), 198-206.

Nesi, H. (2013 a). Dictionary use. In C. A. Chapelle (Ed.), The Encyclopedia of Applied Linguistics. Oxford: Wiley-Blackwell.

Nesi, H. (2013 b). Researching users and uses of dictionaries. In H. Jackson (Ed.), The Bloomsbury companion to lexicography (pp. 62-74). London: Bloomsbury.

Nesi, H., \& Meara, P. (1994). Patterns of misinterpretation in the productive use of EFL dictionary definitions. System, 22(1).

Newport, E. L., \& Bellugi, U. (1978). Linguistic expression of category levels in a visualgestural language: A flower is a flower is a flower. In E. Rosch \& B. B. Lloyd (Eds.), Cognition and categorization (pp. 49-71). Hillsdale, N.J.: Lawrence Erlbaum Associates.

Newport, E. L., \& Meier, R. P. (1985). The acquisition of American Sign Language. In Applied Psycholinguistics (Vol. 1, pp. 881-938).

Nielsen, S. (2008). The effect of lexicographical information costs on dictionary making and use. Lexikos, 18, 170-189.

Nist, S. L., \& Olejnik, S. (1995). The role of context and dictionary definitions on varying levels of word knowledge. Reading Research Quarterly, 30(2), 172 - 193.

Ogilvie, S. (2011). Linguistics, lexicography, and the revitalization of endangered languages. International Journal of Lexicography, 24(4), 389-404.

Okuyama, Y., \& Igarashi, H. (2007). Think-aloud protocol on dictionary use by advanced learners of Japanese. The JALT CALL Journal, 3(1-2), 45-58.

Ow, S. H. (2009). User evaluation of an electronic Malaysian Sign Language dictionary: eSign dictionary. Computer and Information Science, 2(2), 34 - 52.

Padden, C. (1980). The deaf community and the culture of deaf people. In C. Baker \& R. Battison (Eds.), Sign language and the deaf community: Essays in honor of William $C$. Stokoe (pp. 89-104). Silver Spring, MD: National Association of the Deaf.

Padden, C. A. (1983). Interaction of morphology and syntax in American Sign Language. (Unpublished doctoral dissertation). University of California, San Diego.

Padden, C. A., \& Gunsauls, D. C. (2003). How the alphabet came to be used in a sign language. Sign Language Studies, 4(1), 10-33.

Perniss, P., Thompson, R., \& Vigliocco, G. (2010). Iconicity as a general property of language: Evidence from spoken and signed languages. Frontiers in Psychology, 1(227).

Petitto, L. A. (1987). On the autonomy of language and gesture: Evidence from the acquisition of personal pronouns in American sign language. Cognition, 27(1), 1-52. 
Petitto, L. A. (200o). On the biological foundations of human language. In K. Emmorey \& H. Lane (Eds.), The signs of language revisited: An anthology in honor of Ursula Bellugi and Edward Klima. (pp. 447-471). Mahwah, New Jersey: Lawrence Erlbaum Associates.

Pivac Alexander, S. (2008). Sociolinguistic variation in the use of fingerspelling in NZSL. (Unpublished master's dissertation). Victoria University of Wellington, New Zealand.

Pizzuto, E., \& Pietrandrea, P. (2001). The notation of signed texts: Open questions and indications for further research. Sign Language $\mathcal{E}$ Linguistics, 4(1-1), 29-45.

Potgieter, L. (2012). Example sentences in bilingual school dictionaries. Lexikos, 22, 261-271.

Potter, L. (1998). Setting a good example: What kind of examples best serve the users of learners' dictionaries? In T. Fontenelle (Ed.), EURALEX' 98 proceedings: Papers submitted to the eighth EURALEX International Congress on Lexicography (Vol. 1, pp. 357-362). Liege: University of Liege.

Prillwitz, S., Hanke, T., König, S., Konrad, R., Langer, G., \& Schwarz, A. (2008). DGS corpus project: Development of a corpus-based electronic dictionary of German Sign Language / German. In O. Crasborn, E. Efthimiou, T. Hanke, E. Thoutenhoofd, \& I. Zwitserlood (Eds.), Construction and exploitation of sign language corpora. Proceedings of the 3rd Workshop on the representation and processing of sign languages. 6th International Conference on Language Resources and Evaluation, LREC 2008, Marrakech. (pp. 159 - 164). Paris: ELRA.

Prillwitz, S., Leven, R., Zienert, H., Hanke, T., \& Henning, J. (1989). HamNoSys Version 2.o. Hamburg Notation System for sign languages-An introductory guide. Hamburg: Signum.

Prinsloo, D. J. (2012). Electronic lexicography for lesser-resourced languages: The South African context. In S. Granger \& M. Paquot (Eds.), Electronic lexicography (pp. 119-144). Oxford: Oxford University Press.

Prinsloo, D. J., \& Gouws, R. H. (2000). The use of examples in polyfunctional dictionaries. Lexikos, 10, 138-156.

QSR International. (2015). NVIVO qualitative data analysis software for Mac [computer software]. QSR International.

Qualtrics [Computer software]. (2016). Provo, Utah: Qualtrics. Retrieved from http:// www.qualtrics.com.

Quine, W. V. O. (1960). Word and object. Cambridge, Massachusetts: Harvard University Press.

Rathmann, C., \& Mathur, G. (2002). Is verb agreement the same crossmodally? In K. A. Cormier \& D. Quinto-Pozos (Eds.), Modality and structure in signed and spoken languages (pp. 370 - 404). Cambridge: Cambridge University Press.

Reffell, H., \& Locker McKee, R. (2009). Motives and outcomes of New Zealand Sign Language legislation: a comparative study between New Zealand and Finland. Current Issues in Language Planning, 10(3), 272-292. 
Richards, J. C., \& Taylor, A. (1992). Defining strategies in folk definitions. Working papers of the Department of English, City Polytechnic of Hong Kong (Vol. 4). Hong Kong.

Rosch, E. (1973). Natural categories. Cognitive Psychology, 4(3), 328-350.

Rosch, E. (1978). Principles of categorization. In E. Rosch \& B. B. Lloyd (Eds.), Cognition and categorization (pp. 2-48). Hillsdale, N.J.: Lawrence Erlbaum Associates.

Rundell, M. (1999). Dictionary use in production. International Journal of Lexicography, 12(1), 35-53.

Rundell, M. (2006). More than one way to skin a cat: Why full-sentence definitions have not been universally adopted. In T. Fontenelle (Ed.), Proceedings of the XIIth EURALEX International Congress (pp. 323-338). Turin: Universita di Torino.

Sandler, W., \& Lillo-Martin, D. (2006). Sign language and linguistic universals. Cambridge: Cambridge University Press.

Schembri, A. (2003). Rethinking “classifiers” in signed languages. In K. Emmorey (Ed.), Perspectives on classifier constructions in sign languages (pp. 3-34). New York \& London: Taylor \& Francis Group.

Schembri, A., \& Johnston, T. (2004). Sociolinguistic variation in Auslan (Australian Sign Language): A research project in progress. Deaf Worlds, 2o(1), 78-9o.

Schermer, G. M. M. (2001). The role of mouthings in Sign Language of the Netherlands: Some implications for the production of sign language dictionaries. In P. Boyes Braem \& R. Sutton-Spence (Eds.), The hands are the head of the mouth: The mouth as articulator in sign languages (pp. 273-284). Hamburg: Signum-Verlag.

Schermer, G. M. M. (2006). Sign language: Lexicography. In Encyclopedia of Language and Linguistics (Second Edit., pp. 321-324). Amsterdam / London: Elsevier.

Schermer, G. M. M., \& Koolhof, C. (2010). The first national Dutch Sign Language (NGT) Dictionary in book form: Van Dale Basiswoordenboek Nederlandse Gebarentaal. In A. Dykstra \& T. Schoonheim (Eds.), Proceedings of the 14th Euralex International Congress, 6-1oth July 2010 (pp. 1555-1564). Leeuwarden / Ljouwert, The Netherlands: Fryske Akademy.

Schmaling, C. H. (2012). Dictionaries of African sign languages: An overview. Sign Language Studies, 12(2), 236-278.

Simonsen, H. K. (2011). User consultation behaviour in internet dictionaries: An eye-tracking study. Hermes, 46, 75-101.

Sinclair, A. (1986). Metalinguistic knowledge and language development. Advances in Psychology, 39, 609-627.

Sinclair, J. M. (1987 b). Grammar in the dictionary. In J. M. Sinclair (Ed.), Looking up: An account of the COBUILD project in lexical computing (pp. 104-115). London / Glasgow: Collins ELT.

Smith, E. (2003). "Deaf Ways": Literacy teaching strategies of Deaf teachers in New Zealand. (Unpublished masters dissertation). Victoria University of Wellington. 
Smith, N., Tsimpli, I., Morgan, G., \& Woll, B. (2011). The signs of a savant: Language against the odds. Cambridge: Cambridge University Press.

Snell-Hornby, M. (1990). Dynamics in meaning as a problem for bilingual lexicography. In J. Tomaszczyk \& B. Lewandowska-Tomaszczyk (Eds.), Meaning and lexicography (pp. 209226). Amsterdam / Philadelphia: John Benjamins.

Statistics New Zealand. (2013). 2013 Census QuickStats about culture and identity. Retrieved from http://www.stats.govt.nz/Census/2013-census/profile-and-summary-reports/ quickstats-culture-identity/languages.aspx

Stock, P. (1988). The structure and function of definitions. In M. Snell-Hornby (Ed.), ZuriLEX'86 proceedings: Papers read at the EURALEX International Congress, University of Zürich 9-14 September 1986 (pp. 81-89). Tübingen: Francke Verlag.

Stock, P. (1992). The cultural dimension in defining. In H. Tommola (Ed.), EURALEX' 92 proceedings I-II: Papers submitted to the 5th EURALEX International Congress on Lexicography in Tampere, Finland. Tampere: Department of Translation Studies, University of Tampere.

Stokoe, W. C. (1960). Sign language structure: An outline of the visual communication systems of the American deaf. Studies in Linguistics: Occasional Papers No. 8. Buffalo: University of Buffalo.

Summers, D. (1988). The role of dictionaries in language learning. In R. Carter \& M. McCarthy (Eds.), Vocabulary and language teaching (pp. 111 - 125). London / New York: Longman.

Supalla, T. (1982). Structure and acquisition of verbs of motion and location in American Sign Language. (Unpublished doctoral dissertation). University of California, San Diego.

Supalla, T. (1986). The classifier system in American Sign Language. In C. G. Craig (Ed.), Noun classification and categorization (pp. 181-214). Amsterdam / Philadelphia: John Benjamins Publishing Company.

Supalla, T., \& Newport, E. (1978). How many seats in a chair? The derivation of nouns and verbs in American Sign Language. In P. Siple (Ed.), Understanding language through sign language research (pp. 91-132). New York: Academic Press.

Supalla, T., \& Webb, R. (1995). The grammar of International Sign: A new look at pidgin languages. In K. Emmorey \& J. S. Reilly (Eds.), Language, gesture and space (pp. 333354). Hillside, N.J.: Lawrence Erlbaum Associates.

Sutton, V. (2002). Lessons in sign writing: Textbook \& workbook (Third Edit.). La Jolla, CA: Deaf Action Committee for SignWriting.

Sutton-Spence, R. (1999). The influence of English on British Sign Language. International Journal of Bilingualism, 3(4), 363-394.

Sutton-Spence, R., \& Day, L. (2001). Mouthings and mouth gestures in British Sign Language (BSL). In P. Boyes Braem \& R. Sutton-Spence (Eds.), The hands are the head of the mouth: The mouth as articulator in sign languages (pp. 69-85). Hamburg: Signum-Verlag. 
Sutton-Spence, R., \& Woll, B. (1999). The linguistics of British Sign Language: An introduction. Cambridge: Cambridge University Press.

Swanepoel, P. H. (2003). Dictionary typologies: A pragmatic approach. In P. van Sterkenburg (Ed.), A practical guide to lexicography (pp. 44 - 70). Amsterdam: John Benjamins Publishing Company.

Tarp, S. (2009). Reflections on lexicographical user research. Lexikos, 19, 275-296.

Taub, S. F. (2001). Language from the body: Iconicity and metaphor in American Sign Language. Cambridge: Cambridge University Press.

Tervoort, B. T. (1953). Structurele analyse van visueel taalgebruik binnen een groep dove kinderen. Amsterdam: Noord-Hollandsche Uitgevers Maatschappij.

Thumb, J. (2004). Dictionary look-up strategies and the bilingualised learner's dictionary: A think aloud study. Lexicographica, Series Maior (Vol. 117). Tübingen: Max Niemeyer Verlag.

Tono, Y. (2001). Research on dictionary use in the context of foreign language learning. Tübingen: Max Niemeyer Verlag.

Tono, Y. (2003). Research on dictionary use: Methodological considerations. In R. R. K. Hartmann (Ed.), Lexicography: Critical concepts. Volume I: Dictionaries, compilers, critics and users (pp. 394-412). London / New York: Routledge.

Troelsgård, T., \& Kristoffersen, J. H. (2008). An electronic dictionary of Danish Sign Language. In R. M. de Quadros (Ed.), Sign Languages: spinning and unraveling the past, present and future. TISLR9, forty five papers and three posters from the gth. Theoretical Issues in Sign Language Research Conference, Florianopolis, Brazil, December 2006. (pp. 652-662). Petropolis / RJ, Brazil: Editora Arara Azul.

van den Bogaerde, B. (200o). Input and interaction in deaffamilies. Utrecht: LOT Publications.

Van Hoek, K. (1992). Conceptual spaces and pronominal reference in American Sign Language. Nordic Journal of Linguistics, 15(2), $183-199$.

van Sterkenburg, P. (2003). “The” dictionary: Definition and history. In P. van Sterkenburg (Ed.), A practical guide to lexicography (pp. 3-17). Amsterdam: John Benjamins Publishing Company.

Varantola, K. (2002). Use and usability of dictionaries: Common sense and context sensibility? In M.-H. Corréard (Ed.), Lexicography and natural language processing: A festschrift in honour of B.T.S. Atkins. Stuttgart: Euralex.

Vermeerbergen, M. (2006). Past and current trends in sign language research. Language and Communication, 26, 168-192.

Vermeerbergen, M., Leeson, L., \& Crasborn, O. (Eds.). (2007). Simultaneity in signed languages: Form and function. Amsterdam: John Benjamins Publishing Company. 
Weinreich, U. (1970). La definition lexicographique dans la semantique descriptive (J. R.-D., Trans.). Langages, (19), 69-86. (Reprinted from Lexicographic definition in descriptive semantics, in Problems in lexicography by F. Householder \& S. Saporta (Eds.), 1967, The Hague: Mouton)

Welker, H. (2010). Dictionary use: A general survey of empirical studies. Brasilia, Brazil: Author's Edition.

Wiegand, H. E. (1977). Nachdenken über Wörterbücher: Aktuelle Probleme. In G. Drosdowski, H. Henne, \& H. E. Wiegand (Eds.), Nachdenken über Wörterbücher (pp. 51 - 102). Mannheim / Vienna / Zürich: Bibliographisches Institut.

Wiegand, H. E. (1998). Wörterbuchforschung. Untersuchungen zur Wörterbuchbenutzung, zur Theorie, Geschichte, Kritik und Automatisierung der Lexikographie. Berlin: Walter de Gruyter.

Wilbur, R. (1979). American Sign Language and sign systems. Baltimore: University Park Press.

Wilcox, P. P. (2000). Metaphor in American Sign Language. Washington, D.C.: Gallaudet University Press.

Wilcox, P. P. (2005). What do you think? Metaphor in thought and communication domains in American Sign Language. Sign Language Studies, 5(3), 267-291.

Wilcox, S. (2003). The Multimedia Dictionary of American Sign Language: Learning lessons about language, technology, and business. Sign Language Studies, 3(4), 379-392.

Wilson, M., \& Emmorey, K. (1997). A visuospatial “phonological loop” in working memory: Evidence from American Sign Language. Memory and Cognition, 25(3), 313-320.

Woll, B. (1998). Development of signed and spoken languages. In S. Gregory, P. Knight, W. McCracken, S. Powers, \& L. Watson (Eds.), Issues in Deaf education (pp. 58-68). London: Fulton.

Woll, B. (2001). The sign that dares to speak its name: Echo phonology in British Sign Language (BSL). In P. Boyes Braem \& R. Sutton-Spence (Eds.), The hands are the head of the mouth: The mouth as articulator in sign languages (pp. 87 - 98). Hamburg: SignumVerlag.

Woodward, J. (1975, March). How you gonna get to heaven if you can't talk with Jesus: The educational establishment vs. the Deaf community. Paper presented at the Annual Meeting of the Society for Applied Anthropology, Amsterdam.

Woodward, J., \& Horejes, T. P. (2016). deaf/Deaf: Origins and usage. In G. Gertz \& P. Boudreault (Eds.), The SAGE Deaf Studies Encyclopedia. Sage Publications.

World Federation of the Deaf (n.d.). Sign language. Retrieved from http://wfdeaf.org/ human-rights/crpd/sign-language

Zeshan, U., \& de Vos, C. (Eds.). (2012). Sign languages in village communities: Anthropological and linguistic insights. Berlin: de Gruyter Mouton.

Zgusta, L. (1971). Manual of lexicography. The Hague: De Gruyter Mouton. 
Zgusta, L. (1987). Translational equivalence in a bilingual dictionary: Bāhukośyam.

Dictionaries: Journal of the Dictionary Society of North America, 9(1), 1-47.

Zgusta, L. (2006). Equivalents and explanations in bilingual dictionaries. In F. F. M. Dolezal \& T. B. I. Creamer (Eds.), Lexicography then and now: Selected essays by Ladislav Zgusta (pp. 230-235). Tübingen: Max Niemeyer. (Reprinted from Linguistic and literary studies in honor of Archibald A. Hill, Vol. IV., by M. A. Jazayery, E. Polomé and W. Winter, eds., 1979, The Hague: Mouton).

Zwitserlood, I. (2010). Sign language lexicography in the early 21st century and a recently published dictionary of Sign Language of the Netherlands. International Journal of Lexicography, 23(4), 443-476.

Zwitserlood, I. (2012). Classifiers. In R. Pfau, M. Steinbach, \& B. Woll (Eds.), Sign language: An international handbook (pp. 158 - 186). Berlin / Boston: De Gruyter Mouton.

Zwitserlood, I., Kristoffersen, J. H., \& Troelsgård, T. (2013). Issues in sign language lexicography. In H. Jackson (Ed.), The Bloomsbury companion to lexicography (pp. 259283). London: Bloomsbury. 